\title{
CONFORMAL DIFFERENTIAL GEOMETRY OF A SUBSPACE
}

\author{
BY \\ AARON FIALKOW
}

TABLE OF CONTENTS

Introduction

\section{Preliminary matters}

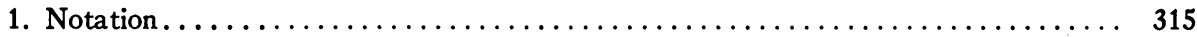

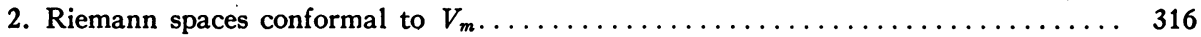

3. Conformal tensors. . . . . . . . . . . . 318

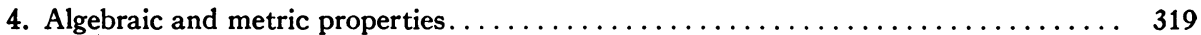

5. Generalized covariant differentiation and the second fundamental form of $V_{n} \ldots \ldots 323$

II. Conformal tensor Algebra

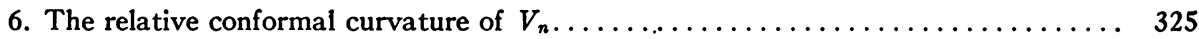

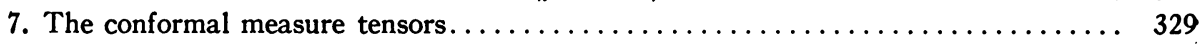

III. Conformal tensor analysis

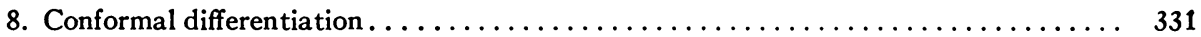

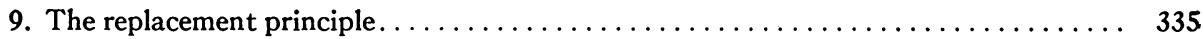

10. Conformal geodesic and conformal normal coordinates and parameters........ 341

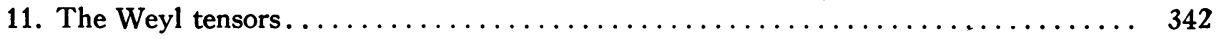

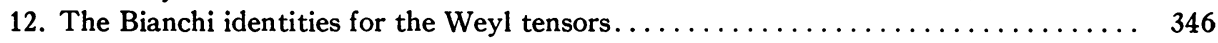

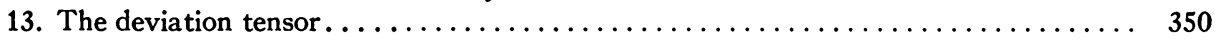

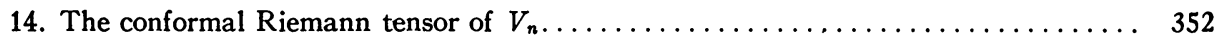

15. Variation of the mean curvature normal $\ldots \ldots \ldots \ldots \ldots \ldots \ldots \ldots \ldots \ldots \ldots \ldots \ldots \ldots \ldots$

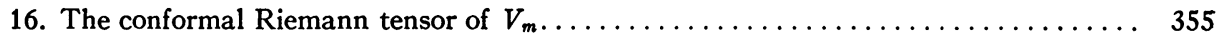

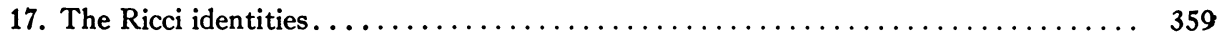

IV. THE CONFORMAL VECTOR SPACES OF $V_{n}$

18. The conformal osculating and normal vector spaces of $V_{n} \ldots \ldots \ldots \ldots \ldots \ldots$

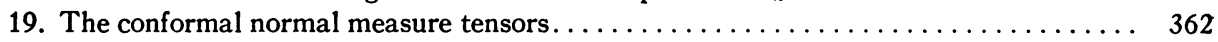

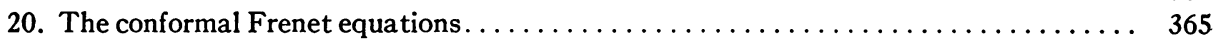

\section{The Fundamental EQuations}

21. The boundary, algebraic, reality and derived conditions............ 370

22. The conformal Gauss-Codazzi equations $\ldots \ldots \ldots \ldots \ldots \ldots \ldots \ldots \ldots \ldots \ldots \ldots$

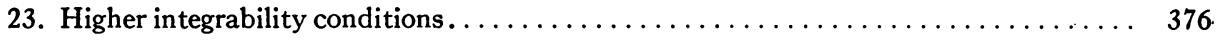

24. The third derivatives of the relative conformal curvature $\ldots \ldots \ldots \ldots \ldots \ldots \ldots \ldots$

25. The second derivatives of the mean curvature. Discussion for a hypersurface..... 383

26. The second derivatives of the mean curvature normal. Discussion for the general case 385

VI. Subspaces in a CONFormally EuClidean SPaCe $\bar{R}_{m}$

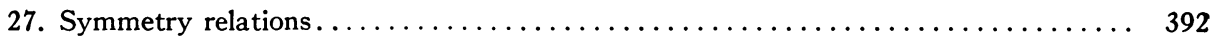

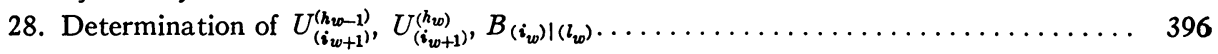

Presented to the Society, October 26, 1940 and February 22, 1941; received by the editors February 1, 1943, and, in revised form, December 15, 1943. 
29. The existence theorem................................... 412

30. Groups of conformal transformations in euclidean space $R_{m}$ and in a conformally euclidean space $\bar{R}_{m}$.

31. The conformal equivalence theorem $\ldots \ldots \ldots \ldots \ldots \ldots \ldots \ldots \ldots \ldots \ldots \ldots, 421$

32. Conformal differential forms $\ldots \ldots \ldots \ldots \ldots \ldots \ldots \ldots \ldots \ldots \ldots \ldots \ldots, 422$

VII. EXceptional SUbSPACES

33. Conformal null subspaces................................. 426

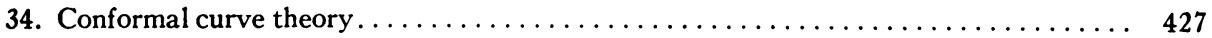

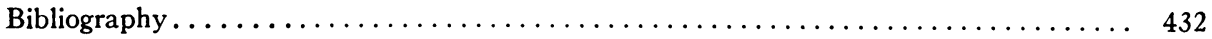

\section{INTRODUCTION}

Scope. Conformal differential geometry (as developed in this paper) concerns itself with certain spaces, point transformations, and configurations. The particular spaces which are considered here are the $m$-dimensional Riemann spaces $V_{m}$. These spaces are generalizations of and include euclidean $m$-space $R_{m}$. The basic point transformations of this paper are the conformal transformations. A conformal transformation defined on a Riemann space $V_{m}$ is a mapping of $V_{m}$ on itself or another Riemann space $\bar{V}_{m}$ so that corresponding angles in $V_{m}$ and $\bar{V}_{m}$ are equal in magnitude. We do not study the conformal transformations of the Riemannian spaces as a whole upon each other (that is, the intrinsic conformal theory of the space) but the conformal geometry of configurations in these spaces. The configuration in $V_{m}$ which we study is a subspace $V_{n}$. The dimensionality $n$ of this subspace may be any integer between 1 (curve) and $m-1$ (hypersurface) inclusive. Accordingly, conformal differential geometry, as defined above, investigates those properties of a curve, hypersurface or other subspace which remain unchanged when the enveloping Riemann space $V_{m}$ undergoes any conformal mapping, not necessarily on itself. As an important special case, this geometry includes the inversive geometry of a euclidean space $R_{m}$.

Method. Euclidean geometry may be defined as the invariant theory of the euclidean metric or congruence group. Not only do the congruence transformations define the geometry; they were a fundamental method in the development of the early (Greek) geometry of the plane. The picture is quite different in the classical differential geometry of two and three-dimensional euclidean space. Here, since the time of Gauss, the fundamental method in the geometry (as distinguished from its definition) has been based upon quadratic differential forms. These quadratic differential forms control the metric of the space and the metrics and curvatures of configurations (curves, surfaces) in the space. What was a powerful tool for the geometric exploration of space in the hands of Gauss became the defining structure of the space in the epoch making generalization of Ricmann. The geometry of Riemann spaces and the corresponding tensor calculus have since been developed by Christoffel, Ricci, Levi-Civita and others into a beautiful and elegant theory. The power of the methods of Riemannian geometry rests primarily upon 
(1) the existence of a fundamental metric tensor (whose components are the coefficients of the fundamental quadratic differential form), (2) the process of covariant differentiation, (3) the tensor character of the important geometric objects in the space, and of the operations performed upon these objects.

In our development of conformal differential geometry, we have largely ignored the conformal transformations which define the geometry and have attempted to take maximum advantage of the methods of Riemannian geometry. A first necessary step in the realization of this aim is the discovery of a symmetric second order tensor in $V_{m}$ (corresponding to the Riemann metric tensor) which remains unchanged by conformal transformations of $V_{m}$. In the intrinsic conformal theory of $V_{m}$, there does not exist at present any method for attaining this desirable objective.

But a subspace $V_{n}$ introduces new elements of structure into the enveloping Riemann space $V_{m}$ at points of $V_{n}$. By means of these new structural properties, we discover a relative conformal scalar $\left.{ }^{1}\right) \Lambda$ which exists at points of the subspace. The existence of $\Lambda$ makes it an easy matter to define two new symmetric second order tensors which do not change when $V_{m}$ undergoes any conformal transformation. These tensors, called the "conformal measure tensors," introduce a new "conformal metric" into the space. The first tensor is used to measure "conformal distance" in the subspace $V_{n}$ and the second to measure "conformal distance" in the enveloping space $V_{m}$ at points of $V_{n}$. By making use of $\Lambda$, it is also possible to invent a new type of covariant differentiation (with respect to the subspace $V_{n}$ ) which plays a role analogous to ordinary covariant differentiation in Riemannian geometry. This "conformal covariant differentiation" process or "conformal derivative" enjoys all the usual properties of covariant differentiation as well as a number of others which give it its distinctive conformal character.

The tensors for the measurement of conformal distance and the conformal differentiation process constitute the methodological foundation for our development of conformal differential geometry. By means of the conformal measure tensors and conformal differentiation, it is possible to apply all the usual operations to conformal tensors $\left({ }^{2}\right)$ without altering either their conformal or tensor character. Consequently our development of conformal differential geometry proceeds by a full utilization of the technical apparatus of tensor calculus and Riemannian geometry. Its formal, technical aspects are scarcely more involved than those of the ordinary (metric) theory of Riemann spaces. The same methods may be used to investigate the conformal differential geometry of configurations $\left(^{3}\right)$ in $V_{m}$ other than those which are considered here.

(1) This term is defined in $\S 3$ and $\Lambda$ is defined in $\$ 6$.

(2) This term is defined in $\$ 3$.

(3) Examples of such configurations are: congruences of curves, families of subspaces, $m$-beins. 
The formal calculations themselves may be shortened considerably by making use of the "replacement principle." This principle states that certain tensors which appear frequently in the sequel may be replaced by zeros in the expressions where they occur. It depends upon a change in gauge, but in its effect is somewhat analogous to the choice of special coordinate systems frequently made in classical and Riemannian geometry.

Results. In the preceding paragraphs we have discussed, in general terms, the scope of our subject and the methods employed. What are the actual questions which are considered and what answers are obtained? The concluclusions of this paper may be summarized under the following topics:

(1) Conformal geometric objects. A number of new conformal geometric objects are invented and their definition obtained in tensor form. Among these geometric objects are conformal analogues of the metric tensor, the Christoffel symbols, and the Riemann tensor, all of which are defined both for the subspace $V_{n}$ and for the enveloping space $V_{m}$ at points of $V_{n}$. We also construct various new tensors from the Weyl conformal curvature tensor. The existence of conformal analogues of the geodesic and normal coordinates of Riemannian geometry is proved. By successive conformal differentiation of the tangent vector space of $V_{n}$, we obtain a sequence of conformal vector spaces analogous to the osculating and normal vector spaces of $V_{n}$ in Riemannian geometry. The measurement of the conformal length of vectors lying in these "conformal normal vector spaces" introduces various tensors analogous to the coefficients of the second fundamental form of a hypersurface. We call these tensors the "conformal normal measure tensors" of $V_{n}$. We also define still another symmetric second order tensor in connection with the variation of the relative conformal curvature $\Lambda$. This tensor which we call the "deviation tensor" has no analogue in Riemannian geometry. Symmetric tensors constructed from the various conformal measure tensors and the deviation tensor are the "conformal fundamental tensors" of $V_{n}$. Their components serve as coefficients of the various "conformal fundamental forms" of $V_{n}$.

(2) Conformal relations. Various relations among these conformal geometric objects (principally in the form of conformal tensor equations) are derived. For example, both the Weyl tensors and the "conformal Riemann tensors" have symmetry properties like those of the ordinary Riemann tensor and satisfy identities similar to the Bianchi identities of Riemannian geometry. Conformal differentiation of the various conformal tangent and normal vector spaces leads to a system of tensor equations analogous to the Frenet equations. The integrability conditions of these "conformal Frenet equations" are another system of equations involving various conformal tensors. The simplest of these latter equations are analogues of the Gauss equations and the Codazzi equations which occur both in classical and Riemannian geometry. In addition to the above conditions, the integrability conditions of still another set of partial differential equations which have no analogue in classi- 
cal or Riemannian geometry are of equal importance. However, if $n>2$ some of these last conditions, and if $n>3$ all of these last conditions, are consequences of the integrability conditions of the conformal Frenet equations. The various integrability conditions enumerated above as well as a number of other important algebraic and differential equations are termed the "fundamental equations" of $V_{n}$. All of the quantities which occur in the fundamental equations are conformal tensors defined on $V_{n}$.

(3) The basic theorems. Since the process of construction of the conformal fundamental forms of $V_{n}$ consisted entirely of conformal elements, it follows that if $V_{m} \rightleftarrows \bar{V}_{m}, V_{n} \rightleftarrows \bar{V}_{n}$ by a conformal map, then the corresponding fundamental forms of $V_{n}$ and $\bar{V}_{n}$ are equal. The converse holds in spaces which are conformal to a euclidean space. In this case, we have the fundamental conformal equivalence theorem: If $V_{m}$ and $\bar{V}_{m}$ are conformal to a euclidean space and the corresponding conformal fundamental forms of $V_{n}$ and $\bar{V}_{n}$ are equal, then a conformal transformation exists so that $V_{m} \rightleftarrows \bar{V}_{m}$ and $V_{n} \rightleftarrows \bar{V}_{n}$. This is the conformal analogue of the congruence theorem of classical and Riemannian geometry which holds in a euclidean space and in a space of constant curvature. We also prove the existence theorem: $A$ subspace $V_{n}$ exists in any conformally euclidean space with preassigned conformal fundamental forms whose coefficients satisfy the fundamental equations. The $V_{n}$ is determined uniquely by a set of initial conditions which is found explicitly. The basic question concerning the conformal differential forms of any subspace $V_{n}$ is answered (at least when the enveloping space is conformally euclidean) by the theorem: The coefficients of any conformal differential form of $a V_{n}$ in a conformally euclidean space are tensor functions of the conformal fundamental tensors and the conformal Riemann tensor of $V_{n}$ and their conformal derivatives, and conversely.

(4) Exceptional cases. If the relative conformal curvature $\Lambda$ vanishes identically in $V_{n}$, all the results outlined above become meaningless. There are two categories of exceptional cases for which $\Lambda \equiv 0$ :

(i) $n>1$. In this case, the conformal distance measured between any two points on $V_{n}$ is identically zero and $V_{n}$ is a "conformal null subspace." These subspaces retain this characteristic under conformal transformations of $V_{m}$. They are the conformal images of totally geodesic subspaces, and conversely. In euclidean space, they are the $n$-dimensional planes and spheres.

(ii) $n=1$. The conformal theory outlined in this introduction does not apply to one-dimensional subspaces since $\Lambda$ is identically zero on every curve. The conformal geometry of curves was developed by the author in a separate paper [8] $\left({ }^{4}\right)$. Results analogous to those stated above were proved subject to the restriction that the dimensionality $\left(^{5}\right) m$ of the enveloping space ex-

(4) The numbers in brackets refer to the bibliography at the end of the paper.

$(5)$ This is a natural restriction since every analytic curve in a $V_{2}$ is conformally equivalent to a straight line in the plane and hence cannot have conformal properties. 
ceed 2. If $m=2$, the results still apply if the conformal transformations are restricted to mappings which are similar to and include the inversive transformations of the plane. In the last section of this paper, we show how these results of our previous paper on conformal curve theory may be obtained by a slight modification of the methods used here for a general subspace.

Previous literature $\left.{ }^{6}\right)$. The literature on conformal differential geometry may be divided into two main categories. One approach to the subject is the intrinsic conformal theory; that is, the investigation of conformal transformations of Rierrann spaces as a whole upon each other. The discovery of the conformal curvature tensor by Weyl and the characterization of conformally euclidean spaces by Schouten mark the modern beginning of this subject. The central problem of the intrinsic theory is the question of the conformal equivalence of Riemann spaces. A number of investigations by T. Y. Thomas, Veblen, Cartan, Schouten, Haantjes and others have appeared which deal with this topic.

The other approach (which is followed in this paper) is the study of configurations in an enveloping Riemann space $V_{m}$ and their behavior when the enveloping manifold undergoes any conformal mapping. An early result of this kind is the theorem of Schouten and Struik which states that the lines of curvature of a hypersurface of $V_{m}$ remain invariant under conformal transformations of $V_{m}$. Several other results of a similar character exist, some of which are not purely conformal theorems since they depend upon metric properties of the configuration and upon the particular transformation to which the $V_{m}$ is subjected. Recently a number of papers by Sasaki and Yano have appearcd which investigate the conformal geometry of subspaces using the formal methods which have been developed in connection with the intrinsic theory.

In addition to the above work, a considerable number of papers have appeared dealing with the inversive geometry of plane curves and of curves and surfaces in euclidean three-dimensional space. These studies make no use of tensors or of Riemannian geometry; their methods depend, in most cases, upon the Schwarzian derivative or tetracyclic and pentaspherical coordinates. These investigations of inversive geometry are the only papers on conformal geometry which in any way overlap the work of the present paper. In particular, the books of Thomsen and Blaschke [2] and of Takasu [14] constitute an inversive theory of curves and surfaces in the plane and in euclidean 3-space which is complete in its essential parts and anticipates many of our results for this important but special case. However, their methods depend upon the systematic use of tetracyclic and pentaspherical coordinates and therefore differ completely from the methods which are employed here.

${ }^{(6)}$ References to and more detailed remarks about the various papers mentioned in this section appear in our paper on curve theory [8, pp. 436-438, 441, 495]. Also see the book of Schouten and Struik [13, pp. 199-215] for a general discussion of the conformal differential geometry of Riemann spaces. 


\section{Preliminary matters}

1. Notation. In this section, various conventions concerning notation and terminology which are used in the sequel are summarized. Except where the contrary is stated explicitly, it is understood that these conventions are used throughout the paper. Precise definitions of the various terms used in this section appear in later portions of the paper.

Terminology. (i) In this paper, two different metrics occur: the usual Riemann metric of Riemannian geometry and the new metric of conformal geometry. The Riemannian metric of a space is frequently referred to simply as the "metric" of the space and geometric objects which are constructed relative to the Riemannian metric are referred to as "metric geometric objects."

(ii) However, when no ambiguity is possible, the adjective "conformal" used in referring to various geometric objects is omitted. For example, the "conformal measure tensors" are usually called the "measure tensors."

(iii) The words "space" and "surface" are used to distinguish geometric objects defined relative to an enveloping Riemann space or to a subspace imbedded in it. When there is no danger of ambiguity, these words are omitted. For example, the "surface conformal Riemann tensor" may be referred to as the "conformal Riemann tensor."

General conventions. (i) The usual conventions of tensor notation are assumed. For example, a tensor equation in which an index is not summed is valid for each value of the index within its range. When the same letter appears in any term as a subscript and superscript, it is understood that this letter is summed for all values within its range. Sometimes summation is also denoted by $\sum$.

(ii) The same kernel letter $\left({ }^{7}\right)$ is used to indicate the contravariant and covariant components of the same vector. For example, $\pi^{\alpha}$ and $\pi_{\alpha}$ refer to the same vector. A similar assumption is made for any tensor.

(iii) Space and surface components of the same tensor are also indicated by the same kernel letter. For example, the space vector $\lambda^{\alpha}$ and the space tensor $\pi_{\beta l}^{\alpha k}$ have the surface components $\lambda^{i}$ and $\pi^{\imath k}{ }_{l l}$ respectively.

(iv) The properties of a general tensor are usually illustrated by means of the tensor $\pi^{\alpha}{ }_{\beta}$ or $\pi_{j}^{i}$ or $\pi^{\alpha i}{ }_{\beta j}$. Here $\alpha$ and $i$ ( $\beta$ and $j$ ) represent any contravariant (covariant) space and surface indices respectively.

(v) As a general rule, lower case Roman and Greek kernel letters represent Riemannian metric geometric objects, capital Greek kernel letters represent relative conformal geometric objects and capital Roman kernel letters represent conformal geometric objects.

(vi) References, indicated by brackets, refer to the bibliography at the end of the paper.

${ }^{(7)}$ We call $\pi$ the "kernel letter" of the vector $\pi^{\alpha}$ or $\pi_{\alpha}$ and use similar terminology for any tensor. 
Indices. The set of indices $\alpha, \beta, \gamma, \delta, \epsilon, \zeta ; g, h, i, j, k, l ; \tau, \omega$ have the ranges $1,2, \cdots, m ; 1,2, \cdots, n ; n+1, n+2, \cdots, m$ respectively. The powers and subscripts $u, v, w, a, b$ have arbitrary ranges. Indices such as $\alpha_{w}$ and $i_{v}$ have the same range as $\alpha$ and $i$ respectively. The symbol $\left(i_{w}\right)$ means $i_{1} i_{2} \cdots i_{w}$. The letters $m, n, p$ and $q, r, s$ represent dimension numbers and class numbers respectively. They are not indices.

Spaces. The symbols $V_{m}, R_{m}, S_{m}$ are used to represent an $m$-dimensional Riemann space, euclidean space, and space of constant curvature respectively. The symbol $V^{(p)}$ refers to a $p$-dimensional vector space. However $I_{1}, I_{12}, \cdots, I_{12} \cdots M$ and $I_{2}, I_{3}, \cdots, I_{M}$ refer to the conformal osculating vector spaces and conformal normal vector spaces of $V_{n}$.

Conformal correspondence. A Riemann space conformal to $V_{m}$ is denoted by $\bar{V}_{m}$. Thus $\bar{R}_{m}$ signifies a conformally euclidean space. A geometric object in $\bar{V}_{m}$ corresponding to the geometric object $\mathcal{F}$ in $V_{m}$ is denoted by $\overline{\mathcal{F}}$.

Operations. (i) The projection of a vector $\pi^{\alpha}$ in a vector space $V^{(p)}$ is denoted by ${ }^{*} \pi^{\alpha}$. The precise space $V^{(p)}$ into which $\pi^{\alpha}$ is projected is indicated in the context.

(ii) Covariant differentiation with respect to $a_{\alpha \beta}, g_{i j}$ and $\bar{a}_{\alpha \beta}, \bar{g}_{i j}$ are denoted by a comma (,) and semicolon (;) respectively. The symbol for conformal covariant differentiation is the colon $(:)$. The conformal derivative (along a curve) with respect to $S$ is denoted by $\vartheta / \vartheta S$.

(iii) A cyclic sum of tensors is indicated by a plus sign $(+)$ and an alternating sum by a minus sign ( - ). For example,

$$
\begin{aligned}
& \rho_{\beta \gamma \delta}^{\alpha}+\rho_{\delta \gamma \beta}^{\alpha} \equiv \rho^{\alpha+\gamma \delta^{+}}, \quad B_{i j} F_{k}-B_{i k} F_{j} \equiv B_{i j}-F_{k^{-}} \equiv B_{i(j} F_{k)^{-}}, \\
& \rho^{\alpha}{ }_{\beta \gamma \delta}+\rho^{\alpha}{ }_{\gamma \delta \beta}+\rho^{\alpha}{ }_{\delta \beta \gamma} \equiv \rho_{\beta^{+} \gamma^{+\delta^{+}}}^{\alpha} \equiv \rho_{\beta^{+}(\gamma \delta)^{+}}^{\alpha} \equiv \rho^{\alpha{ }_{(\beta \gamma \delta)^{+}}}, \\
& \rho_{\beta \gamma \delta, \epsilon}^{\alpha}+\rho_{\beta \delta \epsilon, \gamma}^{\alpha}+\rho_{\beta \epsilon \gamma, \delta}^{\alpha} \equiv \rho_{\beta \gamma^{+} \delta^{+}, \epsilon^{+}}^{\alpha} \equiv \rho_{\beta(\gamma \delta)^{+}, \epsilon^{+}}^{\alpha} \equiv \rho_{\beta(\gamma \delta, \epsilon)^{+}}^{\alpha} \text {, } \\
& G^{\alpha_{i j k}}-G^{\alpha}{ }_{i k j} \equiv G^{\alpha_{i j^{-} k^{-}}} \equiv G^{\alpha}{ }_{i(j k)^{-}} \text {, } \\
& E_{i j: k}-E_{i k: j} \equiv E_{i j^{-}: k^{-}} \equiv E_{i(j: k)^{-}} \text {. }
\end{aligned}
$$

(iv) The replacement operation is denoted by a small zero $\left(^{0}\right)$. For example, ${ }^{0} \kappa_{i j},{ }^{0} a_{\alpha \beta}$.

(v) The Weyl operation is symbolized by a vertical arrow ( $\uparrow$ ). For example, $\uparrow \rho^{\alpha}{ }_{\beta \gamma \delta}$.

2. Riemann spaces conformal to $V_{m}$. Let $V_{m}$ be a real Riemann space whose coordinate manifold is of $\operatorname{class}\left({ }^{8}\right) C^{q}$ and whose real metric tensor, defined over the manifold, is positive definite $\left({ }^{9}\right)$ and of class $C^{q-1}$ with $q \geqq 1$.

( $\left.{ }^{8}\right)$ The definitions of the class of a coordinate manifold and of a Riemann space are based upon the discussion which appears in [16, pp. 98-99, 104]. In particular, if the coordinate manifold is of class $C^{q}$, then the admissible coordinate systems are related to each other by transformations of class $C^{q}$.

( ${ }^{2}$ ) The greater part of the following discussion and of the results of the paper will hold even if the metric tensor is indefinite provided that it is not singular. The only real novelty arises when a vector is a null vector. We shall not consider the indefinite case. 
Briefly, we say that $V_{m}$ is a Riemann space of class $C^{q}$. Suppose $\left\{y^{\alpha}\right\}$ are admissible real local coordinates in a coordinate neighborhood of any point of $V_{m}$. In each coordinate neighborhood, we write the first fundamental form of $V_{m}$ as

$$
d s^{2}=a_{\alpha \beta} d y^{\alpha} d y^{\beta} .
$$

Let $\bar{V}_{m}$ be a real Riemann space of class $C^{q}$ whose first fundamental form may be written as

$$
d \bar{s}^{2}=\bar{a}_{\alpha \beta} d \bar{y}^{\alpha} d \bar{y}^{\beta}
$$

where $\left\{\bar{y}^{\alpha}\right\}$ are allowable local coordinates. Then $\bar{V}_{m}$ is conformal to $V_{m}$ by means of a transformation of class $C^{q}$ (briefly: $\bar{V}_{m}$ is conformal to $V_{m}$ ) if a oneto-one point transformation $\mathcal{T}$ exists between the points $\mathcal{P}$ of $V_{m}$ and the points $\overline{\boldsymbol{P}}$ of $\bar{V}_{m}$ which may be written (locally) as

$$
\bar{y}^{\alpha}=\bar{y}^{\alpha}\left(y^{1}, y^{2}, \cdots, y^{m}\right), \quad y^{\alpha}=y^{\alpha}\left(\bar{y}^{1}, \bar{y}^{2}, \cdots, \bar{y}^{m}\right)
$$

so that the real functions

$$
\bar{y}^{\alpha}\left(y^{1}, y^{2}, \cdots, y^{m}\right), \quad y^{\alpha}\left(\bar{y}^{1}, \bar{y}^{2}, \cdots, \bar{y}^{m}\right)
$$

are of class $C^{q}$ and $\left({ }^{10}\right)$

$$
d \bar{s}=e^{\sigma} d s
$$

at corresponding points. It follows that $\sigma\left(y^{\alpha}\right)$ is a real function of class $C^{\alpha-1}$ and that the form (2.2) is positive definite. We refer to $\sigma\left(y^{\alpha}\right)$ as the conformal mapping function of $V_{m}$ on $\bar{V}_{m}$, or briefly, as the mapping function. Whenever we say that $\bar{V}_{m}$ is conformal to $V_{m}$ it is to be understood that the conformal transformation is of class $C^{q}$.

The transformation $\mathcal{T}$ may be written in the simple form

$$
\bar{y}^{\alpha}=y^{\alpha}
$$

after a suitable change of coordinates. For if we transform the coordinate neighborhoods of $V_{m}$ according to (2.3) considered as an admissible coordinate transformation, points in $\bar{V}_{m}$ and $V_{m}$ with the same coordinates correspond and the conformal transformation becomes (2.5). Throughout this paper, unless a contrary assumption is explicitly made, we shall always assume that coordinate systems have been chosen so that (2.5) holds. In these coordinate systems,

$$
\bar{a}_{\alpha \beta}=e^{2 \sigma} a_{\alpha \beta}, \quad \bar{a}^{\alpha \beta}=\bar{e}^{2 \sigma} a^{\alpha \beta},
$$

where $a^{\alpha \beta}$ and $\bar{a}^{\alpha \beta}$ are the contravariant components of the metric tensors.

(10) The condition (2.4) is readily seen to be equivalent to either of the geometric statements: (1) The magnitudes of corresponding angles of $V_{m}$ and $\bar{V}_{m}$ are equal. (2) The ratio of the length of any vector at any point $P$ of $V_{m}$ to the length of the corresponding vector in $\bar{V}_{m}$ is the same for all vectors at $\mathcal{P}$; that is, the transformation is a local similitude. 
The contravariant metric tensor $a^{\alpha \beta}$ is determined by the equations

$$
a_{\beta \gamma} a^{\alpha \gamma}=\delta^{\alpha}{ }_{\beta}
$$

where $\delta^{\alpha}{ }_{\beta}$ are the Kronecker deltas and $\bar{a}^{\alpha \beta}$ is determined by similar equations. Conversely, if (2.5) is a point transformation of the points of $V_{m}$ and $\bar{V}_{m}$ and (2.6) holds at corresponding points where the mapping function $\sigma\left(y^{\alpha}\right)$ is a real function of class $C^{q-1}$, it follows that $\bar{V}_{m}$ is conformal to $V_{m}$.

Let

$$
\left\{e^{2 \sigma} a_{\alpha \beta}\right\}
$$

denote the set of all second order, symmetric, positive definite tensors of class $C^{q-1}$, any two of which are equal except for a positive multiplicative scalar factor of class $C^{q-1}$. Let

$$
\left\{\overline{\mathrm{V}}_{m}\right\}
$$

denote the set of all conformally equivalent Riemann spaces of class $C^{q}$ whose metric tensors (in some allowable coordinate system) belong to (2.7). Throughout this paper, whenever we refer to the conformally equivalent Riemann spaces $V_{m}, \bar{V}_{m}$, it will be understood that these spaces are any two spaces of the set of conformally equivalent Riemann spaces (2.8).

3. Conformal tensors. Let $T_{\beta_{1} \ldots \beta_{v}}^{\alpha_{1} \ldots \alpha_{w}}$ be components of a tensor at a point $\Phi$ of $V_{m}$ whose values depend upon geometric objects of $V_{m}$ and of its subspaces $\left.{ }^{11}\right)$. Let $\bar{V}_{m}$ be any Riemann space conformal to $V_{m}$ and let $\bar{T}_{\beta_{1} \ldots \beta_{v}}^{\alpha_{1} \ldots \alpha_{v}}$ be the components of the tensor at $\bar{P}$ whose values depend in the same way upon the corresponding geometric objects of $\bar{V}_{m}$ and its corresponding subspaces. Then if (2.6) holds and

$$
\bar{T}_{\beta_{1} \cdots \beta_{v}}^{\alpha_{1} \cdots \alpha_{v}}=\left(e^{\sigma}\right)^{u} T_{\beta_{1} \cdots \beta_{v}}^{\alpha_{1} \cdots \alpha_{w}}
$$

we call $T_{\beta_{1} \ldots \beta_{v}}^{\alpha_{1} \ldots \alpha_{v}}$ a relative conformal tensor of weight $u$. The law of transformation of $T_{\beta_{1} \ldots \beta_{v}}^{\alpha_{1} \ldots \alpha_{w}}$ between any two $\bar{V}_{m}$ as well as between any two coordinate systems is consistent. If $u=0$, the tensor has the same components in $V_{m}$ and $\bar{V}_{m}$. In this case, we call $T_{\beta_{1} \ldots \beta_{v}}^{\alpha_{1} \ldots \alpha_{w}}$ a conformal tensor.

As a consequence of our definitions it follows that if the components of a relative conformal tensor are zero in $V_{m}$, they are zero in any $\bar{V}_{m}$. This fact permits us to write conformal tensor equations which retain their meaning under conformal transformations. The sum or difference of relative conformal tensors of the same weight and valence is a relative conformal tensor of that weight and valence. The inner or outer product of relative conformal tensors is a relative conformal tensor. In particular, the sum, difference, inner or outer product of conformal tensors is a conformal tensor.

(11) Examples of such geometric objects which will be used in this paper are: the metric tensor $a_{\alpha \beta}$, the Christoffel symbols of the second kind, the coefficients of the first and second fundamental forms of a subspace. 
As examples, we note that the metric tensors $a_{\alpha \beta}$ and $a^{\alpha \beta}$ are relative conformal tensors of weight 2 and -2 respectively as follows from (2.6). The Weyl conformal curvature tensor $C^{\alpha}{ }_{\beta \gamma \delta}$ is a conformal tensor.

4. Algebraic and metric properties. In $\$ \$ 4,5$, we summarize some of the results of the metric theory of a subspace of a Riemann space. These well known results are useful for the development of the conformal theory. We suppose that $V_{m}$ is any Riemann space belonging to (2.8) and let $R$ be a subset of $V_{m}$ with the following properties:

(a) $R$ is a real $n$-dimensional topological space with $n<m$.

(b) The coordinate manifold of $R$ is of class $C^{r}$ where $r$ is a fixed integer subject to the inequalities

$$
g \geqq r
$$

and $\left({ }^{12}\right)$

$$
r \geqq 1 \text {. }
$$

We denote any system of admissible local coordinates in a coordinate neighborhood of $R$ by $\left\{x^{i}\right\}$. Following Mayer [3, p. 53], we refer to the coordinates $x^{i}$ of a point of $R$ as parameters to differentiate them from the coordinates $y^{\alpha}$ of the same point considered as a point of $\left({ }^{13}\right) V_{m}$.

(c) In each parameter neighborhood of $R$, lying in a coordinate neighborhood of $V_{m}$, the correspondence between the coordinates $y^{\alpha}$ and the parameters $x^{i}$ at points of $R$ determines the $y^{\alpha}$ as functions of class $C^{r}$ in the $x^{i}$; that is, the functions

$$
y^{\alpha}=y^{\alpha}\left(x^{1}, x^{2}, \cdots, x^{n}\right)
$$

are of class $C^{r}$. Equations (4.3) are the parametric equations of $R$. It follows from (b) that condition (c) is true in every allowable coordinate and parameter system. Hence if a tensor, defined on a space neighborhood containing a surface neighborhood, is of class $C^{s}(s \leqq r)$ in the $y^{\alpha}$ it is also of class $C^{s}$ in the $x^{i}$.

(d) The rank of the matrix $\left\|\partial y^{\alpha} / \partial x^{i}\right\|$ at a given fixed point $P$ of $R$ is $n$. Since, according to (4.2), the functions $\partial y^{\alpha} / \partial x^{i}$ are continuous, it follows that the rank of $\left\|\partial y^{\alpha} / \partial x^{i}\right\|$ must be $n$ at each point $\mathcal{P}^{\prime}$ of a sufficiently small neigh-

(12) It is assumed that conditions (a) to (d) hold throughout this paper except that (4.2) is frequently replaced by a stronger inequality.

(13) Similarly, we shall distinguish quantities and geometric objects defined relative to $V_{m}$ and to $R$ from each other by means of the words "space" and "surface" respectively. For example, a space tensor (surface tensor) exhibits its tensor character with respect to transformations of the coordinates $y^{\alpha}$ (parameters $x^{i}$ ) and remains unchanged under parameter (coordinate) transformations. Space components of a tensor are denoted by indices having the range $1,2, \cdots, m$ (space indices); surface components by indices having the range $1,2, \cdots, n$ (surface indices). A tensor defined at a point of $R$ which has both space and surface indices may be represented uniquely in terms of two beins-an $m$-bein spanning $V_{m}$ and an $n$-bein spanning $\mathcal{R}$. 
borhood $U(P)$ of the point $P$. If $\left\{y^{\prime \alpha}\right\},\left\{x^{\prime i}\right\}$ are allowable coordinate and parameter systems,

$$
\frac{\partial y^{\prime \alpha}}{\partial x^{\prime i}}=\frac{\partial y^{\alpha}}{\partial y^{\beta}} \cdot \frac{\partial y^{\beta}}{\partial x^{j}} \cdot \frac{\partial x^{j}}{\partial x^{\prime i}}, \quad \frac{\partial y^{\alpha}}{\partial x^{i}}=\frac{\partial y^{\alpha}}{\partial y^{\prime \beta}} \cdot \frac{\partial y^{\prime \beta}}{\partial x^{\prime j}} \cdot \frac{\partial x^{\prime j}}{\partial x^{i}} .
$$

Hence it is clear that (d) holds independently of any allowable choice of coordinates and parameters. It also follows from (4.4) that $\partial y^{\alpha} / \partial x^{i}$ is a contravariant vector with respect to coordinate changes (space transformations) and a covariant vector with respect to parameter changes (surface transformations). We frequently write $y_{i}^{\alpha}$ for $\partial y^{\alpha} / \partial x^{i}$.

According to (4.4),

$$
\partial y^{\alpha} / \partial x^{1}, \partial y^{\alpha} / \partial x^{2}, \cdots, \partial y^{\alpha} / \partial x^{n}
$$

defined at each point $\Phi^{\prime} \subset \mathcal{U}(\Phi)$, represent $n$ linearly independent contravariant vectors with respect to changes of the coordinates $y^{\alpha}$. These vectors span the tangent vector space of $R$ at $\Phi^{\prime}$. If $\lambda^{\alpha}$ and $\lambda^{i}$ are space and surface components of any vector in the tangent vector space of $R$ at $\Phi^{\prime}$,

$$
\lambda^{\alpha}=y^{\alpha}{ }_{i} \lambda^{i} \text {. }
$$

The totality of vectors $\zeta^{\alpha}$ which obey the equations

$$
y^{\alpha}{ }_{i} \zeta_{\alpha}=0
$$

form a covariant linear vector space of dimension $m-n$ which is called the normal vector space of $R$ at $\boldsymbol{P}^{\prime}$. Each vector in the normal vector space is called a normal rector.

The equations

$$
g_{i j}=a_{\alpha \beta} y^{\alpha}{ }_{i} y_{j}
$$

define a covariant symmetric positive definite tensor of the second order with respect to parameter transformations. If $\lambda^{\alpha}, \xi^{\alpha}$ are the space components of two contravariant tangent vectors and $\lambda^{i}, \xi^{i}$ are the corresponding surface components of these vectors, then

$$
a_{\alpha \beta} \lambda \alpha \xi^{\beta}=g_{i j} \lambda i \xi^{i} .
$$

Since $g_{i j}$ is not singular, the contravariant components $g^{i j}$ are uniquely defined by means of the tensor equations

$$
g_{i j} g^{j k}=\delta^{k}
$$

where the $\delta$ 's are the Kronecker deltas. If $\lambda_{\alpha}, \lambda_{i}$ are the covariant components of $\lambda^{\alpha}, \lambda^{i}$ defined by $\lambda_{\alpha}=a_{\alpha \beta} \lambda^{\beta}, \lambda_{i}=g_{i j} \lambda^{i}$ then

$$
\lambda_{i}=\lambda_{\alpha} y^{\alpha}{ }_{i} \text {. }
$$

As a consequence of (4.6), the lengths of and angles between contravariant 
tangent vectors may be measured by means of their surface components and the tensor $g_{i j}$. Consequently the metric (2.1) of $V_{m}$ induces a Riemannian metric, by means of the surface metric tensor $g_{i j}$, at all points of the region $V(P)$. As follows from (b), (c) and (4.5), $g_{i j}$ is of class $C^{r-1}$ in the $x^{i}$. Hence the region $U(P)$ is an $n$-dimensional Riemann space $V_{n}$ of class $C^{r}$.

Since the results of this paper are local theorems which hold for a sufficiently small neighborhood of a point, we shall restrict ourselves to a portion of $V_{n}$ which is a surface neighborhood $V_{1}(P)$ of the point $P$ coverable by a single parameter system $\left\{x^{i}\right\}$. Furthermore, we choose $V_{1}(P)$ so that it lies in a space neighborhood $\bigcup_{2}(\Phi)$ coverable by a single coordinate system $\left\{y^{\alpha}\right\}$. We shall refer to $U_{1}(P)$ and $U_{2}(P)$ as the Riemann spaces $V_{n}$ and $V_{m}$ respectively and use similar language in connection with the other Riemann spaces which appear in the paper.

Let $\pi^{\alpha}, \pi_{\alpha}$ be the contravariant and covariant components of any space vector at a point of $V_{m}$, and $V^{(p)}$ a $p$-dimensional vector space at this point. Then ${ }^{*} \pi^{\alpha},{ }^{*} \pi_{\alpha}$ is called the projection of $\pi^{\alpha}, \pi_{\alpha}$ in $V^{(p)}$ if $(1){ }^{*} \pi^{\alpha},{ }^{*} \pi_{\alpha}$ lies in $V^{(p)},(2) \pi^{\alpha}-{ }^{*} \pi^{\alpha}, \pi_{\alpha}-{ }^{*} \pi_{\alpha}$ is orthogonal $\left({ }^{14}\right)$ to the vector space $V^{(p)}$. A unique symmetric tensor $s^{\alpha \beta}$ always exists so that the projection ${ }^{*} \pi^{\alpha}$ of any $\pi_{\alpha}$ in the fixed $V^{(p)}$ is given by

$$
{ }^{*} \pi^{\alpha}=s^{\alpha \beta} \pi_{\beta} .
$$

The tensor $s^{\alpha \beta}$ as well as the covariant tensor $s_{\alpha \beta}\left(=a_{\alpha \gamma} a_{\beta \delta} s^{\gamma \delta}\right)$ and the mixed tensor $s^{\alpha}\left(=a_{\beta \delta} s^{\alpha \delta}\right)$ is called the projection tensor of the vector space $V^{(p)}$. The vector $\pi^{\alpha}$ may be decomposed into its projection ${ }^{*} \pi^{\alpha}$ in $V^{(p)}$ and another vector $\zeta^{\alpha}$ orthogonal to $V^{(p)}$ so that

$$
\pi^{\alpha}=\pi^{\alpha}+\zeta^{\alpha} .
$$

A canonical representation of $s^{\alpha \beta}$ exists by means of a normalized $p$-bein (that is, a set of $p$ mutually orthogonal unit vectors) which spans $V^{(p)}$. If ${ }_{(1)} \lambda^{\alpha},{ }_{(2)} \lambda^{\alpha}, \cdots,(p) \lambda^{\alpha}$ is this $p$-bein then

$$
s^{\alpha \beta}={ }_{(1)} \lambda^{\alpha}{ }_{(1)} \lambda^{\beta}+{ }_{(2)} \lambda^{\alpha}{ }_{(2)} \lambda^{\beta}+\cdots+{ }_{(p)} \lambda^{\alpha}{ }_{(p)} \lambda^{\beta} .
$$

In particular, if ${ }_{(1)} \lambda^{\alpha},{ }_{(2)} \lambda^{\alpha}, \cdots,{ }_{(n)} \lambda^{\alpha}$ is a normalized $n$-bein which spans the tangent vector space of $V_{n}$ at a point and if ${ }_{(1)} \zeta^{\alpha},{ }_{(2)} \zeta^{\alpha}, \cdots,{ }_{(m-n)} \zeta^{\alpha}$ is a normalized $(m-n)$-bein spanning the normal vector space of $V_{n}$ at this point, then

$$
\begin{aligned}
a^{\alpha \beta}= & { }_{(1)} \lambda^{\alpha}{ }_{(1)} \lambda^{\beta}+{ }_{(2)} \lambda^{\alpha}{ }_{(2)} \lambda^{\beta}+\cdots+{ }_{(n)} \lambda^{\alpha}{ }_{(n)} \lambda^{\beta}+{ }_{(1)} \zeta^{\alpha}{ }_{(1)} \zeta^{\beta}+{ }_{(2)} \zeta^{\alpha}{ }_{(2)} \zeta^{\beta} \\
& +\cdots+{ }_{(m-n)} \zeta^{\alpha}{ }_{(m-n)} \zeta^{\beta} \\
g^{i j}= & { }_{(1)} \lambda^{i}{ }_{(1)} \lambda^{j}+{ }_{(2)} \lambda^{i}{ }_{(2)} \lambda^{j}+\cdots+{ }_{(n)} \lambda^{i}{ }_{(n)} \lambda^{j} \\
h^{\alpha \beta}= & { }_{(1)} \zeta^{\alpha}{ }_{(1)} \zeta^{\beta}+{ }_{(2)} \zeta^{\alpha}{ }_{(2)} \zeta^{\beta}+\cdots+{ }_{(m-n)} \zeta^{\alpha}{ }_{(m-n)} \zeta^{\beta}
\end{aligned}
$$

(14) A vector space $V^{\left(p_{1}\right)}$ is orthogonal to a vector space $V^{\left(p_{2}\right)}$ if each vector in $V^{\left(p_{1}\right)}$ is orthogonal to every vector in $V^{\left(p_{2}\right)}$. 
where $h^{\alpha \beta}$ is the projection tensor of the normal vector space of $V_{n}$. The expression for the projection tensor $g^{\alpha \beta}$ of the tangent vector space at a point of $V_{n}$ is

$$
g^{\alpha \beta}=g^{i j} y^{\alpha}{ }_{i}^{\beta}{ }_{j} .
$$

We write

$$
g^{\alpha}{ }^{\alpha}=a_{\beta \delta} \delta^{\alpha \delta}, \quad g_{\alpha \beta}=a_{\alpha \gamma} a_{\beta \delta} g^{\gamma \delta}
$$

and note that $g^{\alpha}{ }_{i}$ defined as $g^{\alpha}{ }_{\beta} y^{\beta}{ }_{i}$ may be written as

$$
g^{\alpha}{ }_{i}=y^{\alpha}{ }_{i}=\partial y^{\alpha} / \partial x^{i} .
$$

If $h^{\alpha \beta}, h^{\alpha}{ }_{\beta}, h_{\alpha \beta}$ represent the various components of the projection tensor of the normal vector space of $V_{n}$,

$$
h^{\alpha \beta}=a^{\alpha \beta}-g^{\alpha \beta}, \quad h_{\beta}^{\alpha}=\delta^{\alpha} \beta-g^{\alpha}{ }_{\beta}, \quad h_{\alpha \beta}=a_{\alpha \beta}-g_{\alpha \beta} .
$$

If (4.8) represents the decomposition of a space vector $\pi^{\alpha}$ relative to the tangent vector space, then we have

$$
\pi_{\alpha} g^{\alpha_{i}}={ }^{*} \pi_{\alpha} g^{\alpha}{ }_{i}={ }^{*} \pi_{i}
$$

where ${ }^{*} \pi_{i}$ are the surface components of ${ }^{*} \pi_{\alpha}$.

The projection of any tensor in the tangent vector space may be defined in a manner analogous to that used for the space vector $\pi^{\alpha}$. We first give some preliminary definitions. A space index $\alpha$ of a tensor $\left({ }^{15}\right) \pi^{\alpha}, \pi_{\alpha}$ is called a tangent index if

$$
\pi^{\alpha} \zeta_{\alpha}=0, \quad \pi_{\alpha} \zeta^{\alpha}=0
$$

for every normal vector $\zeta^{\alpha}, \zeta_{\alpha}$. The name tangent index is also applied to every surface index of the tensor. Any tangent space index may be raised or lowered by means of $g^{\alpha \beta}, g_{\alpha \beta}$ instead of $a^{\alpha \beta}, a_{\alpha \beta}$. A tangent tensor is a tensor all of whose indices are tangent indices. For example, the projection tensor $g_{\alpha \beta}$ is a tangent tensor. Any tangent tensor may be represented in the usual manner by an $n$-bein (having both space and surface components) spanning the tangent vector space. The surface components $\lambda^{k t}{ }_{j j}$ of any tangent tensor $\lambda^{\alpha t}{ }_{\beta j}$ are obtained by a formula like that of equations (4.7) which holds for tangent vectors. The formula is

$$
\lambda^{k i}{ }_{l j}=\lambda^{\alpha i}{ }_{\beta j} g^{k}{ }_{\alpha} g^{\beta}{ }_{l}
$$

where $g^{\beta}{ }_{l}$ and $g_{\alpha}^{k}$ are defined by (4.11) and (4.14) respectively.

The mixed tensor ${ }^{*} \pi^{\alpha t}{ }_{\beta j}$ is the projection of $\pi^{\alpha t}{ }_{\beta j}$ in the tangent vector space of $V_{n}$ if (1) ${ }^{*} \pi^{\alpha t}{ }_{\beta j}$ is a tangent tensor, (2) $\pi^{\alpha t}{ }_{\beta j}-{ }^{*} \pi^{\alpha t}{ }_{\beta j}$ is not a tangent tensor

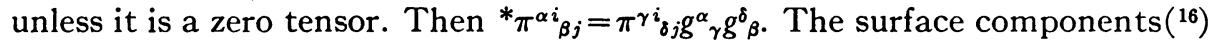

(15) We have omitted all tensor indices except the index $\alpha$ to which reference is made.

(16) When there is no danger of ambiguity, we write $\pi^{k i}$ for ${ }^{*} \pi_{i j}^{k i}$. 
${ }^{*} \pi^{k i}{ }_{l j}$ of the projection ${ }^{*} \pi^{\alpha i}{ }_{\beta j}$ are obtained by means of the equations

$$
{ }^{*} \pi^{k i}{ }_{l j}=\pi^{\alpha i}{ }_{\beta j} g_{\alpha}^{k} g^{\beta}{ }_{l} .
$$

We note some algebraic relations which were useful in the above development and are used in the sequel. These equations are either definitions or simple consequences of the definitions of the various tensors.

$$
\begin{aligned}
g^{\alpha i} & =g^{\alpha}{ }_{j} g^{i j}, \quad g^{i}{ }_{\alpha}=a_{\alpha \beta} g^{\beta i}, \quad g_{\alpha i}=a_{\alpha \beta} g^{\beta}{ }_{i}, \\
a^{\alpha \gamma} a_{\beta \gamma} & =a^{\alpha}{ }_{\beta}=\delta^{\alpha}, \\
g^{i}{ }^{i k} g_{k j} & =g^{\alpha}{ }_{j} g^{i}{ }_{\alpha}=g^{\alpha i} g_{\alpha j}=g^{i}{ }_{j}=\delta^{i}{ }_{j}, \\
a^{\alpha}{ }_{\beta} g^{\beta}{ }_{i} & =g^{\alpha}{ }_{\beta} g^{\beta}{ }_{i}=g^{\alpha}{ }_{j} g^{j}{ }_{i}=g^{\alpha i} g_{i j}=g^{\alpha \beta} g_{\beta i}=g^{\alpha}{ }_{i}, \\
h^{\alpha} g_{\alpha} & =h_{\alpha} g^{\alpha}=0 .
\end{aligned}
$$

In the last set of equations, $g_{\alpha}$ indicates any of the tensors $g_{\alpha}^{\beta}, g_{\alpha}^{i}, g_{\alpha \beta}, g_{\alpha i}$. There is a similar convention for $g^{\alpha}$. Similarly $h^{\alpha}$ indicates either of the tensors $h^{\alpha \beta}, h^{\alpha}$. Finally, we remark that the operations of projection and addition (or substraction) applied to tensors of the same valence are commutative. Whenever the operations of projection and differentiation are applied to a tensor, it is assumed that differentiation follows projection unless the contrary is explicitly indicated.

5. Generalized covariant differentiation and the second fundamental form of $V_{n}$. We replace (4.2) by the stronger inequality

$$
r \geqq 2 \text {. }
$$

Then, according to (4.1) and (5.1), the Christoffel symbols of the second kind for $V_{m}$ and $V_{n}$ exist and are continuous. These symbols are denoted by

$$
\left\{\begin{array}{c}
\alpha \\
\beta \gamma
\end{array}\right\} \text { and }\left\{\begin{array}{c}
i \\
j k
\end{array}\right\}
$$

respectively. The generalized covariant derivative (with respect to $a_{\alpha \beta}$ and $g_{i j}$ ) of a tensor of class $C^{1}$ defined on a surface neighborhood of $V_{n}$ is

$$
\begin{gathered}
\pi^{\alpha i_{\beta j, k}}=\frac{\partial \pi^{\alpha i_{\beta j}}}{\partial x^{k}}+\pi^{\delta i}{ }_{\beta j}\left\{\begin{array}{c}
\alpha \\
\delta \gamma
\end{array}\right\} g^{\gamma_{k}}-\pi^{\alpha i}{ }_{\delta j}\left\{\begin{array}{c}
\delta \\
\beta \gamma
\end{array}\right\} g^{\gamma}{ }_{k} \\
+\pi^{\alpha l}{ }_{\beta i}\left\{\begin{array}{c}
i \\
l k
\end{array}\right\}-\pi^{\alpha i_{\beta l}}\left\{\begin{array}{c}
l \\
j k
\end{array}\right\} .
\end{gathered}
$$

As a consequence of (5.1) and (4.11), the class of $g^{\alpha}{ }_{i}$ is at least 1. Hence its covariant derivative exists and is defined by

$$
g^{\alpha_{i, j}}=\frac{\partial g^{\alpha}{ }_{i}}{\partial x^{j}}+\left\{\begin{array}{c}
\alpha \\
\beta \gamma
\end{array}\right\} g^{\beta}{ }_{i} g_{j}-\left\{\begin{array}{c}
l \\
i j
\end{array}\right\} g^{\alpha}{ }_{l}
$$


whence $g^{\alpha, j}=g^{\alpha}{ }_{j, i}$. We write $g^{\alpha}{ }_{i j}$ for $g^{\alpha}{ }_{i, j}$. The $g^{\alpha}{ }_{i j}$, considered as space vectors, all lie in the normal vector space at any point $P$ of $V_{n}$, that is,

$$
a_{\alpha \beta} g^{\alpha}{ }_{i j} g^{\beta}{ }_{k}=0 .
$$

If $m=n+1$, the dimension of the normal vectors $g^{\alpha}{ }_{i j}$ does not exceed unity so that $g^{\alpha}{ }_{i j}$ may be written as

$$
g^{\alpha}{ }_{i j}=b_{i j} \zeta^{\alpha}
$$

where $b_{i j}$ is a second order symmetric surface tensor and $\zeta^{\alpha}$ is the unit normal vector. The components of the tensor $b_{i j}$ are the coefficients of the second fundamental form of $V_{n}$. If $m>n+1$, the coefficients of the second fundamental form of $V_{n}$ aie the components of the tensor $b_{i j \mid k l}$ defined by

$$
b_{i j \mid k l}=a_{\alpha \beta} g^{\alpha_{i j} g^{\beta} k l} \text {. }
$$

This tensor is equal to $b_{i j} b_{k l}$ if $m=n+1$.

The principal curvature vectors or directions (directions of the lines of curvature) at a point $\mathcal{P}$ of a hypersurface are the principal directions determined by $b_{i j}$; that is, they are the unit vectors ${ }_{(h)} \lambda^{i}$ which satisfy

$$
\left(b_{i j}-{ }_{(h)} b g_{i j}\right)_{(h)} \lambda^{i}=0
$$

where the scalar ${ }_{(h)} b$ is a principal normal curvature of $V_{n}$ at $P$, being determined as a root of the determinant equation

$$
\left|b_{i j}-{ }_{(h)} b g_{i j}\right|=0 .
$$

Since $g_{i j}$ is definite $\left({ }^{17}\right)$, there exists a normalized $n$-bein of principal curvature directions. According to (5.6), the value of ${ }_{(h)} b$ is given by

$$
{ }_{(h)} b=b_{i j(h)} \lambda^{i}{ }_{(h)} \lambda^{j} .
$$

In the case of a subspace with $m>n+1$, there is no unique set of principal curvatures and principal curvature directions. However, there exists an $n$-bein of principal curvature vectors and $n$ principal normal curvatures at each point $\mathcal{P}$ corresponding to any direction $\zeta^{\alpha}$ in the normal vector space of $V_{n}$. These are determined by equations analogous to (5.6), (5.7) and (5.8), with $b_{i j}$ replaced by $g^{\alpha}{ }_{i j} \zeta_{\alpha}$. If every direction at $P$ is a principal curvature direction, the point $P$ is called umbilical.

The mean curvature normal $\mu^{\alpha}$ is defined at any point $\mathcal{P}$ of $V_{n}$ as

$$
\mu^{\alpha}=n^{-1} g^{\alpha}{ }_{i j} g^{i j} .
$$

The length of this vector is called the mean curvature $\mu$. Hence, according to (5.5) and (5.9),

$$
\mu^{2}=n^{-2} b_{i j \mid k l} l^{i j} g^{k} .
$$

(17) See [4, pp. 107-113] for the theory of the principal directions determined by a symmetric covariant tensor of the second order. The present discussion is based upon this theory. 
For a hypersurface, (5.9) and (5.10) may be written as

$$
\mu^{\alpha}=\mu \zeta^{\alpha}, \quad \mu=n^{-1} b_{i j} g^{i j} .
$$

If we make use of the canonical representation of $g_{i j}$ given by (4.9) and also (5.8) and (5.11), we obtain

$$
\mu=n^{-1} \sum_{h}(h) b .
$$

An analogous formula for $\mu$ obtains for any subspace in terms of the principal normal curvatures of $V_{n}$ corresponding to the direction of $\mu^{\alpha}$.

If (5.1) is strengthened still further by the inequality

$$
r \geqq 3 \text {, }
$$

the Riemann curvature tensors $\rho_{\alpha \beta \gamma \delta}$ of $V_{m}$ and $\kappa_{i j k l}$ of $V_{n}$ exist and are of class $C^{q-3}$ and $C^{r-3}$ respectively. The tensors $\rho^{\alpha}{ }_{\beta \gamma \delta}$ and $\kappa^{i}{ }_{j k l}$ are defined by the equations

$$
\begin{aligned}
\rho^{\alpha}{ }_{\beta \gamma \delta} & =\frac{\partial}{\partial y^{\gamma}}\left\{\begin{array}{c}
\alpha \\
\beta \delta
\end{array}\right\}-\frac{\partial}{\partial y^{\delta}}\left\{\begin{array}{c}
\alpha \\
\beta \gamma
\end{array}\right\}+\left\{\begin{array}{c}
\epsilon \\
\beta \delta
\end{array}\right\}\left\{\begin{array}{c}
\alpha \\
\epsilon \gamma
\end{array}\right\}-\left\{\begin{array}{c}
\epsilon \\
\beta \gamma
\end{array}\right\}\left\{\begin{array}{c}
\alpha \\
\epsilon \delta
\end{array}\right\}, \\
\kappa^{i}{ }_{j k l} & =\frac{\partial}{\partial x^{k}}\left\{\begin{array}{c}
i \\
j l
\end{array}\right\}-\frac{\partial}{\partial x^{l}}\left\{\begin{array}{c}
i \\
j k
\end{array}\right\}+\left\{\begin{array}{c}
h \\
j l
\end{array}\right\}\left\{\begin{array}{c}
i \\
h k
\end{array}\right\}-\left\{\begin{array}{c}
h \\
j k
\end{array}\right\}\left\{\begin{array}{c}
i \\
h l
\end{array}\right\} .
\end{aligned}
$$

It is an immediate consequence of these definitions that $\rho_{\alpha \beta \gamma \delta}$ satisfies the identities

$$
\rho_{\alpha \beta \gamma \delta}=-\rho_{\beta \alpha \gamma \delta}=-\rho_{\alpha \beta \delta \gamma}=\rho_{\gamma \delta \alpha \beta}, \quad \rho_{\alpha(\beta \gamma \delta)^{+}}=0
$$

and that $\kappa_{i j k l}$ obeys a similar set of equations. In writing these equations we have represented the cyclic operation by a plus sign and (below) the alternation operation by a minus sign. The Ricci tensor $\rho_{\beta \gamma}$ and the invariant curvature $\rho$ of $V_{m}$ are defined by $\rho_{\beta \gamma}=\rho_{\alpha \beta \gamma \delta} a^{\alpha \delta}, \rho=\rho_{\beta \gamma} a^{\beta \gamma}$ with analogous definitions for $\kappa_{j k}$ and $\kappa$. Under these conditions, the Gauss equations

$$
b_{i(k \mid l)-j}=\kappa_{i j k l}-\rho_{i j k l}
$$

are satisfied where, in accordance with $\S 4$, we have written

$$
\rho_{\alpha \beta \gamma \delta} g^{\alpha}{ }_{i} g^{\beta}{ }_{i}{ }^{\gamma}{ }_{k} g^{\delta}{ }_{l}=\rho_{i j k l} \text {. }
$$

\section{Conformal tensor Algebra}

6. The relative conformal curvature of $V_{n}$. Let $\bar{V}_{m}$ be any other Riemann space belonging to (2.8) and let $\bar{R}$ be the subset of $\bar{V}_{m}$ which correspond to $R$ under the conformal map (2.4). We suppose that the parameters $\bar{x}^{i}$ at points of $R$ are related to the parameters $x^{i}$ at the corresponding points of $R$ by transformations of class $C^{r}$. Then the assumptions of $\$ 2$ concerning the classes of $V_{m}$ and $\bar{V}_{m}$ and of the conformal mapping between them together with the assumptions (a) to (d) of $\S 4$ about $R$ guarantee that these latter conditions 
also hold true for $\overline{\mathbb{R}}$. Also, according to the discussion of $\S 2$, it is possible to choose allowable parameter systems $\left\{x^{i}\right\}$ and $\left\{\bar{x}^{i}\right\}$ in the parameter neighborhoods of $R$ and $\bar{R}$ respectively so that the transformation of $R$ on $\bar{R}$ is given by $\bar{x}^{i}=x^{i}$. Unless the contrary is stated, we suppose that this is done throughout the remainder of the paper. Then the equations of $\bar{R}$ corresponding to (4.3) are

$$
\bar{y}^{\alpha}=\bar{y}^{\alpha}\left(\bar{x}^{1}, \bar{x}^{2}, \cdots, \bar{x}^{n}\right),
$$

where $y^{\alpha}\left(x^{i}\right)$ and $\bar{y}^{\alpha}\left(\bar{x}^{i}\right)$ are the same functions of $x^{i}$ and $\bar{x}^{i}$ respectively.

According to the discussion of $\$ 4$, the region of $R$ which corresponds to $V_{n}$ is also a Riemann space which we denote by $\bar{V}_{n}$. The remarks of the previous paragraph show that

$$
\bar{g}^{\alpha}{ }_{i}=g^{\alpha}{ }_{i}
$$

where we have written $\bar{g}^{\alpha}{ }_{i}=\partial \bar{y}^{\alpha} / \partial \bar{x}^{i}$. Consequently the tangent vector space of $V_{n}$ is conformally invariant. Since orthogonality is a conformal property, the normal vector spaces of $V_{n}$ and $\bar{V}_{n}$ at corresponding points are also in conformal correspondence. If the metric tensor of $\bar{V}_{n}$ is denoted by $\bar{g}_{i j}, \bar{g}^{i j}$, it follows from equations analogous to (4.5) and from (2.6) and (6.1) that

$$
\bar{g}_{i j}=e^{2 \sigma} g_{i j}, \quad \bar{g}^{i j}=e^{-2 \sigma} g^{i j} .
$$

Thus the conformal transformation (2.4) induces a conformal mapping on the respective subspaces of $V_{m}$ and $\bar{V}_{m}$.

Under the assumption (5.1), the existence and continuity of the Christoffel symbols of the second kind of $\bar{V}_{m}$ and $\bar{V}_{n}$ are assured. We denote these symbols by

$$
\left\{\begin{array}{c}
\bar{\alpha} \\
\beta \gamma
\end{array}\right\} \text { and }\left\{\begin{array}{c}
\bar{i} \\
j k
\end{array}\right\}
$$

respectively. As a consequence of (2.6) and (6.2), we have

$$
\begin{aligned}
& \left\{\begin{array}{c}
\bar{\alpha} \\
\beta \gamma
\end{array}\right\}=\left\{\begin{array}{c}
\alpha \\
\beta \gamma
\end{array}\right\}+a^{\alpha}{ }_{\beta} \sigma_{, \gamma}+a^{\alpha}{ }_{\gamma} \sigma_{, \beta}-a_{\beta \gamma} a^{\alpha \delta_{\sigma, \delta}}, \\
& \left\{\begin{array}{c}
\bar{i} \\
j k
\end{array}\right\}=\left\{\begin{array}{c}
i \\
j k
\end{array}\right\}+g^{i}{ }_{j} \sigma_{, k}+g^{i}{ }_{k} \sigma_{, j}-g_{j k} g^{i l} \sigma_{, l} .
\end{aligned}
$$

We denote covariant differentiation with respect to $\bar{a}_{\alpha \beta}, \bar{g}_{i j}$ by means of a semicolon. Then

$$
\bar{g}^{\alpha_{i ; j}}=\frac{\partial}{\partial \bar{x}^{j}} \bar{g}^{\alpha_{i}}+\left\{\begin{array}{c}
\bar{\alpha} \\
\beta_{\gamma}
\end{array}\right\} \bar{g}^{\beta} \bar{g}^{\gamma}{ }_{i}-\left\{\begin{array}{c}
\bar{l} \\
i j
\end{array}\right\} \bar{g}^{\alpha}{ }_{l} .
$$

We write $\bar{g}^{\alpha}{ }_{i ; j}$ as $\bar{g}^{\alpha_{i j}}$. Also,

$$
\sigma_{, i}=\sigma_{, \alpha} g^{\alpha}{ }_{i}
$$


If we simplify (6.4) by means of (4.5), (4.14), (5.3), (6.1), (6.3) and (6.5), we obtain

$$
\bar{g}^{\alpha_{i j}}=g^{u_{i j}}-g_{i j}\left(a^{\alpha \beta} \sigma_{, \beta}-g^{k l} \sigma_{, k} g^{\alpha}{ }_{l}\right) .
$$

But according to (4.10), (4.12) and (6.5), these last equations may be written as

$$
\bar{g}^{\alpha}{ }_{i j}=g^{\alpha}{ }_{i j}-g_{i j} h^{\alpha \beta} \sigma_{, \beta} .
$$

We denote the mean curvature normal of $\bar{V}_{n}$ by $\bar{\mu}^{\alpha}$. Since

$$
\bar{\mu}^{\alpha}=n^{-1} \bar{g}^{\alpha}{ }_{i j} \bar{g}^{i j},
$$

we find from these equations and (5.9), (6.2) and (6.6) that

$$
e^{2 \sigma} \bar{\mu}^{\alpha}=\mu^{\alpha}-h^{\alpha \beta} \sigma_{, \beta} .
$$

From these equations and $\bar{\mu}_{\alpha}=\bar{a}_{\alpha \beta} \bar{\mu}^{\beta}, \mu_{\alpha}=a_{\alpha \beta} \mu^{\beta}$, we obtain

$$
\bar{\mu}_{\alpha}=\mu_{\alpha}-h_{\alpha}^{\beta} \sigma_{, \beta} \text {. }
$$

As result of (6.2) and (6.7), equations (6.6) become

$$
\bar{G}^{\alpha}{ }_{i j}=G^{\alpha}{ }_{i j}
$$

where we have written

$$
G^{\alpha_{i j}}=g^{\alpha}{ }_{i j}-g_{i j} \mu^{\alpha}
$$

and analogous equations for $\bar{G}^{\alpha_{i j}}$. Consequently the mixed tensor $G^{\alpha_{i j}}$ of class $C^{r-2}$ is a conformal tensor and the contravariant vector space which it spans remains unchanged by conformal transformations. According to (5.4) and (5.9), this vector space is normal to the tangent vector space of $V_{n}$.

If $n=1, V_{n}$ is a curve. In this case the surface metric tensor has one component which may be written as $g_{11}=\lambda_{1} \lambda_{1}$. Then, from (5.9) and (6.10),

$$
G^{\alpha}{ }_{11}=g^{\alpha}{ }_{11}-\lambda_{1} \lambda_{1} g^{\alpha}{ }_{11} \lambda^{1} \lambda^{1}
$$

so that $G^{\alpha}{ }_{i j} \equiv 0$ for a curve. If $G^{\alpha}{ }_{i j}=0$ at a point $\Phi$ of $V_{n}$ and $n$ exceeds unity, it follows from (6.10) that

$$
g^{\alpha}{ }_{i j}=g_{i j} \mu^{\alpha} .
$$

In accordance with the analogue of (5.6) for any subspace, every direction at $\Phi$ is a principal curvature direction so that $P$ is an umbilical point of $V_{n}$. Conversely, if $\mathscr{P}$ is an umbilical point, then (6.11) holds so that $G^{\alpha_{i j}}=0$ at $P$.

The scalar quantity $\Lambda$ defined, except for a choice of sign, by

$$
\Lambda^{2}=n^{-1} a_{\alpha \beta} g^{i l_{g}{ }^{j k} G^{\alpha}{ }_{i j} G^{\beta}{ }_{k l}}
$$

is called the relative conformal curvature of $V_{n}$ if $n>1$. If $n=1, \Lambda$ is identically zero. As follows from (2.6), (6.2) and (6.9), it is a relative conformal scalar of class $C^{r-2}$ obeying the equation 


$$
\bar{\Lambda}=e^{-\sigma} \Lambda \text {. }
$$

The reality of $\Lambda$ is guaranteed by showing that $\Lambda^{2}$, defined by (6.12), is nonnegative. To prove this statement, we choose coordinates $y^{\alpha}$ and parameters $x^{i}$ so that $a_{\alpha \beta}=\delta^{\alpha}{ }_{\beta}, g_{i j}=\delta_{j}^{i}$ at the point $\mathcal{P}$. Then, from (6.12), we have at $\mathcal{P}$ that

$$
\Lambda^{2}=n^{-1} \sum_{\alpha} g^{i l} g^{j k} G^{\alpha}{ }_{i j} G^{\alpha}{ }_{k l}=n^{-1} \sum_{\alpha} \sum_{i} \sum_{j}\left(G^{\alpha}{ }_{i j}\right)^{2} \geqq 0 .
$$

The equality sign is valid if and only if $G^{\alpha}{ }_{i j}=0$. We summarize these results in the following theorem.

THEOREM 6.1. The relative conformal curvature $\Lambda$ of a subspace $V_{n}$ in $V_{m}$ vanishes at a point of $V_{n}$ if and only if the conformal tensor $G^{\alpha}{ }_{i j}$ is zero at the point. If $n=1, G^{\alpha}{ }_{i j}$ vanishes identically. If $n>1, G^{\alpha}{ }_{i j}$ equals zero at a point of $V_{n}$ if and only if this point is umbilical in $V_{m}$.

Throughout the remainder of the paper, except where the contrary is explicitly stated, we shall assume that $\left.{ }^{18}\right) n>1$ (so that $m>2$ ) and restrict ourselves to subspaces $V_{n}$ (more accurately: regions of $V_{n}$ ) which do not contain any umbilical points. Since $\Lambda \neq 0$ in $V_{n}$, we suppose that an initial choice of sign for $\Lambda$ is made so that $\Lambda>0$ in $V_{n}$.

The principal directions determined by the surface tensor $G^{\alpha}{ }_{i j} \zeta_{\alpha}$ where $\zeta^{\alpha}$ is a vector belonging to the normal vector space of $V_{n}$ are, from (6.10), the principal directions determined by $g^{\alpha_{i j}} \zeta_{\alpha}$. Consequently these directions are the principal curvature directions of $V_{n}$ corresponding to $\zeta_{\alpha}$. Since both $G^{\alpha_{i j}}$ and the orthogonality of $\zeta_{\alpha}$ to $V_{n}$ remain unchanged by conformal transformations of $V_{m}$, this proves the conformal invariance of the principal curvature vectors. In particular, we note the following theorems of Schouten and Struik [13, pp. 211-212].

TheOREM 6.2. The lines of curvature of a hypersurface $V_{n}(n>1)$ in $V_{n+1}$ are invariant under conformal transformations of $V_{n+1}$.

THeOREM 6.3. The umbilical points of a subspace $V_{n}(n>1)$ in $V_{m}$ remain umbilical under conformal transformations of $V_{m}$.

Digression. The remainder of this section is devoted to the derivation of some relations between $\Lambda$ and various metric geometric objects of $V_{n}$. The relative conformal curvature may be expressed in terms of the coefficients of the first and second fundamental forms of $V_{n}$. If we make use of equations (4.14), (5.5), (5.9) and (6.10) then (6.12) becomes

$$
\Lambda^{2}=n^{-1} b_{i j \mid k l}\left(g^{i l} g^{j k}-n^{-1} g^{i j} g^{k l}\right) .
$$

If $V_{n}$ is a hypersurface, a formula for $\Lambda$ in terms of the principal normal

(18) The case $n=1$ (surve theory) is considered in $\$ 34$. 
curvatures of $V_{n}$ may be found. In this case, by the use of the canonical representation of $g^{i j}$ given by (4.9) and also (5.8), we find that

$$
b_{i j \mid k l} g^{i l} g^{j k}=\sum_{i}(i) b^{2} .
$$

If we take account of this equation as well as of (5.10) and (5.12), equation (6.14) becomes

$$
\begin{aligned}
\Lambda^{2}=n^{-1}\left[\sum_{i}(i) b^{2}-n^{-1}\left(\sum_{i}(i) b\right)^{2}\right] & =n^{-1} \sum_{i}\left[{ }_{(i)} b^{2}-n^{-1}(i) b \sum_{i}(i) b\right] \\
& =n^{-1} \sum_{i}{ }_{(i)} b[(i) b-\mu] .
\end{aligned}
$$

Now from (5.12),

$$
\sum_{i} \mu[(i) b-\mu]=\mu \sum_{i}\left[{ }_{(i)} b-\mu\right]=0 .
$$

Hence the above equation remains unchanged if $n^{-1} \sum_{i} \mu\left[{ }_{(i)} b-\mu\right]$ is subtracted from the right member. This gives $\left({ }^{19}\right)$

$$
\Lambda^{2}=n^{-1} \sum_{i}\left({ }_{(i)} b-\mu\right)^{2} .
$$

If $m>n+1$, a similar result may be obtained for any subspace $V_{n}$. Let (1) $\zeta^{\alpha}$, (2) $\zeta^{\alpha}, \cdots,(m-n) \zeta^{\alpha}$ be the normalized $(m-n)$-bein of (4.9). We denote the $n$ principal normal curvatures of $V_{n}$ corresponding to (1) $\zeta^{\alpha}$ by $(1,1) b_{1},(1,2) b_{2}, \cdots,(1, n) b_{n}$ and write ${ }_{(1)} \mu=n^{-1} \sum_{i(1, i)} b_{i}$ and use an analogous notation with respect to the other $\zeta^{\alpha}$. In a manner analogous to that used in the derivation of (6.15), we find that

$$
\begin{aligned}
\Lambda^{2}= & n^{-1}\left\{\sum_{i}\left({ }_{(1, i)} b_{i}-{ }_{(1)} \mu\right)^{2}+\sum_{i}\left({ }_{(2, i)} b_{i}-{ }_{(2)} \mu\right)^{2}+\cdots\right. \\
& +\sum_{i}{\left.\left({ }_{(m-n, i)} b_{i}-{ }_{(m-n)} \mu\right)^{2}\right\} .}
\end{aligned}
$$

7. The conformal measure tensors. In this section, we define the fundamental conformal geometric objects which have roles in the conformal theory analogous to those of the metric tensors $a_{\alpha \beta}, g_{i j}$ in classical and Riemannian geometry. If (5.1) is obeyed, the relative conformal curvature $\Lambda$ exists. Since $\Lambda>0$, we may write it as

$$
\Lambda=e^{\phi} .
$$

We call $\phi$ the gauge function of $V_{n}$. According to (2.6), (6.2), (6.13) and (7.1),

(19) An alternative form of equation (6.15) is $\Lambda^{2}=n^{-3} \sum_{i} \sum_{i}((i) b-(j) b)^{2}$. The quantity $(i) b-{ }_{(j)} b$ is a relative conformal scalar having the transformation law $\left[{ }_{(i)} \bar{b}-(j) \bar{b}\right]=e^{-\sigma}\left[{ }_{(i)} b-{ }_{(j)} b\right]$. This fact and the definition of $\Lambda$ given in this footnote provide another proof of (6.13). Analogous remarks apply to the case of a general subspace $V_{n}$ and are based upon equation (6.16) instead of (6.15). 
the tensors $A_{\alpha \beta}, G_{i j}$ defined at points of $V_{n}$ by

$$
\begin{aligned}
A_{\alpha \beta} & =e^{2 \phi} a_{\alpha \beta}, \\
G_{i j} & =e^{2 \phi} g_{i j}
\end{aligned}
$$

are positive definite tensors of class $C^{r-2}$ and remain unchanged by conformal transformations of $V_{m}$, that is,

$$
\bar{A}_{\alpha \beta}=A_{\alpha \beta}, \quad \bar{G}_{i j}=G_{i j} .
$$

We call $A_{\alpha \beta}$ the conformal (space) $\left({ }^{20}\right)$ measure tensor of $V_{m}$ and $G_{i j}$ the conformal (surface) measure tensor of $V_{n}$. Where there is no ambiguity, we shall refer to each of these tensors as a measure tensor.

The contravariant components of these measure tensors are defined by

$$
A^{\alpha \beta} A_{\beta \gamma}=\delta^{\alpha}{ }_{\gamma}, \quad G^{i j} G_{j k}=\delta^{i}{ }_{k} .
$$

It is clear that these tensors satisfy the equations

$$
\begin{aligned}
A^{\alpha \beta} & =e^{-2 \phi} a^{\alpha \beta}, \\
G^{i j} & =e^{-2 \phi} g^{i j} .
\end{aligned}
$$

If $T^{\alpha i}{ }_{\beta j}$ is a conformal tensor and any of its indices are raised or lowered by means of $A^{\alpha \beta}, A_{\alpha \beta}, G^{i j}, G_{i j}$, it is clear that the new tensor retains the conformal character of the original one. Consequently the raising or lowering of the indices of any conformal tensor in the sequel is accomplished by means of these measure tensors unless the contrary is stated. On the other hand, the raising or lowering of the indices of any non-conformal (metric) tensor is done by means of the metric tensors $a^{\alpha \beta}, a_{\alpha \beta}, g^{i j}, g_{i j}$ unless the contrary is indicated. As a result of (4.5), (7.2) and (7.3), the measure tensors are related by the equations

$$
G_{i j}=A_{\alpha \beta} G^{\alpha}{ }_{i} G^{\beta}{ }_{j} .
$$

In these equations, we have written $G^{\alpha}{ }_{i}$ for $\partial y^{\alpha} / \partial x^{i}$ in virtue of the conformal invariance of the latter tensor which is stated in (6.1).

A tensor algebra may be based upon these measure tensors, which is analogous in every respect to the classical algebra developed in $\$ 4$ which is founded upon $a_{\alpha \beta}, g_{i j}$. With reference to conformal tensors similar to those defined in $\$ 4$, every equation of $\$ 4$ has a precise analogue in the new algebra. In particular, the conformal length $T$ of any conformal space vector $T^{\alpha}$ is defined by

$$
T^{2}=A_{\alpha \beta} T^{\alpha} T^{\beta}
$$

(20) It should be noted that the space measure tensor $A_{\alpha \beta}$ is defined relative to the subspace $V_{n}$. This is true of almost all the space conformal objects which appear in this paper and the definitions of these objects should be understood as containing the phase "relative to $V_{n}$." 
If $T=1, T^{\alpha}$ is called a unit conformal vector. If $T^{\alpha}$ is a conformal tangent rector $L^{\alpha}$, its space and surface components obey the equations

$$
L^{\alpha}=L^{i} G^{\alpha}{ }_{i}, \quad L_{i}=L_{\alpha} G^{\alpha}{ }_{i}
$$

and its conformal length may be measured with equal validity by either $A_{\alpha \beta}$ or $G_{i j}$. The magnitude of the angle between two vectors is obviously the same, regardless of whether the angle is measured by means of the metric tensors $a_{\alpha \beta}, g_{i j}$ or the conformal measure tensors $A_{\alpha \beta}, G_{i j}$. This is a natural phenomenon, since the magnitude of an angle (measured by the Riemannian metric) is unchanged by conformal transformations.

For the tangent vector space of $V_{n}$, the projection tensor $G^{\alpha \beta}$ relative to the conformal length measure stated in (7.8) is defined by

$$
G^{\alpha \beta}=G^{i j} G^{\alpha}{ }_{i} G^{\beta}{ }_{j} .
$$

The various equations of $\S 4$ are true if conformal unit vectors are substituted for the metric unit vectors and if the replacements $a \rightarrow A, g \rightarrow G, \pi \rightarrow T, h \rightarrow H$ are made in these equations. In the remainder of the paper, we frequently use the conformal analogue of (4.14) and refer to it simply as equations (4.14). Finally, we note that the important algebraic identities

$$
\begin{aligned}
G^{\alpha}{ }_{i j} G^{i j} & =0, \\
A_{\alpha \beta} G^{\alpha}{ }_{i j} G^{\beta}{ }_{k l} G^{i k} G^{i l} & =n
\end{aligned}
$$

are an easy consequence of (5.9), (6.10), (6.12), (7.1), (7.2), (7.4) and (7.6).

\section{Conformal tensor analysis}

8. Conformal differentiation. In order to define a covariant differentiation process in conformal geometry, we first replace (5.1) by (5.13). This guarantees the existence of the Christoffel symbols. It is natural to require this differentiation process to satisfy the following conditions:

(a) If $T^{\alpha i}{ }_{\beta j}$ is a tensor of class $C^{1}$ defined on a surface neighborhood of $V_{n}$, then $T^{\alpha i}{ }_{\beta j: k}$ may be written as $\left.{ }^{21}\right)$

$$
\begin{aligned}
T^{\alpha i_{\beta j: k}}= & \frac{\partial}{\partial x^{k}} T^{\alpha i_{\beta j}}+T^{\delta i_{\beta j}} \Gamma^{\alpha}{ }_{\delta \gamma} G^{\gamma}{ }_{k}-T^{\alpha i_{\delta j}} \Gamma^{\delta}{ }_{\beta \gamma} G^{\gamma}{ }_{k} \\
& +T^{\alpha l_{\beta j} \Gamma^{i}{ }_{l k}}-T^{\alpha i_{\beta l} \Gamma^{l}{ }_{j k} .}
\end{aligned}
$$

(b) The coefficients $\Gamma_{\beta \gamma}^{\alpha}$ and $\Gamma^{i}{ }_{j k}$, defined $\left({ }^{22}\right)$ on $V_{n}$, are symmetric in the two lower indices.

(21) The form of equations (8.1) is suggested by (5.2). They need not be assumed for a general tensor $T_{\beta j}^{\alpha i}$ but may be developed axiomatically from the assumed laws for a scalar and a contravariant vector. (If $T$ is a scalar, then (8.1) becomes $T_{: k}=\partial T / \partial x^{k}$.) The details of this development are analogous to those of a similar proof in our paper on the conformal theory of curves [8, pp. 447-449].

(22) The definition of $\Gamma_{\beta \gamma}^{\alpha}$ and $\Gamma_{i k}^{i}$ is implicit in conditions (a) to (e). The explicit definition of these coefficients appears in equations (8.5) and (8.11). 
(c) $T^{\alpha i_{\beta j: k}}$ is a tensor.

(d) If $T^{\alpha i}{ }_{\beta j}$ is a conformal tensor, $T^{\alpha i_{\beta j: k}}$ is also a conformal tensor; that is, $\bar{T}^{\alpha i}{ }_{\beta j}=T^{\alpha i}{ }_{\beta j}$ implies $\bar{T}^{\alpha i} i_{j j: k}=T^{\alpha i}{ }_{\beta j: k}$.

(e) The measure tensors behave like constants with reference to the differentiation process; that is,

$$
\begin{aligned}
& A_{\alpha \beta: k}=A_{: k}^{\alpha \beta}=A_{\beta: k}^{\alpha}=0, \\
& G_{i j: k}=G^{i j}: k=G_{j: k}^{i}=0 .
\end{aligned}
$$

The quantities $\Gamma_{\beta \gamma}^{\alpha}$ and $\Gamma^{i}{ }_{j k}$ are the conformal analogues of the Christoffel symbols of the second kind

$$
\left\{\begin{array}{c}
\alpha \\
\beta \gamma
\end{array}\right\} \text { and }\left\{\begin{array}{c}
i \\
j k
\end{array}\right\}
$$

respectively. It follows directly from (a) and (c) that $\Gamma^{\alpha}{ }_{\beta \gamma}$ and $\Gamma^{i}{ }_{j k}$ are coefficients of connection $\left({ }^{23}\right)$ and consequently differ from the Christoffel symbols

$$
\left\{\begin{array}{c}
\alpha \\
\beta \gamma
\end{array}\right\} \text { and }\left\{\begin{array}{c}
i \\
j k
\end{array}\right\}
$$

respectively by quantities which are tensors. The converse is also true. As a result of (a) and (d), it is readily seen that $\Gamma^{\alpha}{ }_{\beta \gamma}$ and $\Gamma^{i}{ }_{j k}$ must be conformal geometric objects which remain unchanged under conformal transformations of $V_{m}$; that is,

$$
\Gamma_{\beta \gamma}=\Gamma_{\beta \gamma}^{\alpha}, \quad \Gamma^{i}{ }_{j k}=\Gamma^{i}{ }_{j k} .
$$

Condition (b) as applied to $\Gamma^{i}{ }_{j k}$ plus the first of equations (8.3) readily determines $\Gamma^{i}{ }_{j k}$ as the Christoffel symbols of the second kind formed from $G_{i j}$. The conformal invariance of these Christoffel symbols may now be proved independently of the above discussion since $G_{i j}$ is a conformal tensor. According to (7.3) and equations similar to (6.3),

$$
\Gamma^{i}{ }_{j k}=\left\{\begin{array}{c}
i \\
j k
\end{array}\right\}+g^{i}{ }_{j} \phi_{, k}+g^{i}{ }_{k} \phi_{, j}-g_{i k} g^{i{ }^{\prime} \phi, l}
$$

The $\Gamma^{\alpha}{ }_{\beta \gamma}$ cannot be similarly determined as the Christoffel symbols formed from $A_{\alpha \beta}$ since $A_{\alpha \beta}$ is only defined at points of $V_{n}$ and consequently $\partial A_{\alpha \beta} / \partial y^{\gamma}$ does not exist. Condition (b) for $\Gamma_{\beta \gamma}^{\alpha}$ and the first of equations (8.2) do not determine $\Gamma_{\beta \gamma}^{\alpha}$ uniquely. If $\Gamma_{\beta \gamma}^{\alpha}$ is chosen in the form

$$
\Gamma_{\beta \gamma}^{\alpha}=\left\{\begin{array}{c}
\alpha \\
\beta \gamma
\end{array}\right\}+a^{\alpha} \pi_{\gamma}+a^{\alpha}{ }_{\gamma} \pi_{\beta}-a_{\beta \gamma} a^{\alpha \delta} \pi_{\delta}
$$

then these conditions are satisfied if $\pi_{\gamma} g^{\gamma}=\phi_{, i}$.

(23) For the definition and properties of coefficients of connection, see [5, pp. 3-6]. 
We digress somewhat in order to find a value for $\pi_{\alpha}$ in this formula which makes (8.6) a conformal geometric object. Equations (6.13) and (7.1) are equivalent to

$$
\sigma=\phi-\phi \text {. }
$$

From this equation, we obtain $\sigma_{, i}=\bar{\phi}_{, i}-\phi_{, i}$. Since $\sigma_{\beta} g_{\alpha}^{\beta_{\alpha}}=\sigma_{\beta} g^{\beta}{ }_{i} g_{\alpha}^{i}=\sigma_{i} g_{\alpha}^{i}$, the above equation leads to

$$
\sigma_{\beta} g_{\alpha}^{\beta}=\phi_{\alpha}-\phi_{\alpha},
$$

where $\phi_{\alpha}, \bar{\phi}_{\alpha}$ are the space components of $\phi_{, i}, \bar{\phi}_{, i}$ so that

$$
\phi_{\alpha}=\phi_{, i} g^{i}{ }_{\alpha} \text {. }
$$

Now

$$
\sigma_{, \alpha}=\sigma_{, \beta} a_{\alpha}^{\beta}=\sigma_{, \beta} g_{\alpha}^{\beta}+\sigma_{, \beta} h_{\alpha}^{\beta} .
$$

The above results and (6.8) permit us to write these last equations in the form

$$
\sigma_{, \alpha}=\eta_{\alpha}-\bar{\eta}_{\alpha}
$$

where $\eta_{\alpha}$ is the gauge vector defined by

$$
\eta_{\alpha}=\phi_{\alpha}+\mu_{\alpha} .
$$

Use of (8.8), (8.9) and (8.10) shows that (6.3) may be $\cdot$ written in the invariantive form

$$
\begin{aligned}
& \left\{\begin{array}{c}
\bar{\alpha} \\
\beta \gamma
\end{array}\right\}+\bar{a}^{\alpha} \bar{\eta}_{\gamma}+\bar{a}^{\alpha}{ }_{\gamma} \bar{\eta}_{\beta}-\bar{a}_{\beta \gamma} \bar{a}^{\alpha \delta} \bar{\eta}_{\delta}=\left\{\begin{array}{c}
\alpha \\
\beta \gamma
\end{array}\right\}+a^{\alpha}{ }_{\beta} \eta_{\gamma}+a^{\alpha}{ }_{\gamma} \eta_{\beta}-a_{\beta \gamma} a^{\alpha \delta} \eta_{\delta}, \\
& \left\{\begin{array}{c}
\bar{i} \\
j k
\end{array}\right\}+\bar{g}^{i}{ }_{j} \bar{\phi}_{, k}+\dot{\bar{g}}^{i}{ }_{k} \bar{\phi}_{, j}-\bar{g}_{j k} \bar{g}^{i}{ }^{l} \bar{\phi}_{, l}=\left\{\begin{array}{c}
i \\
j k
\end{array}\right\}+g^{i}{ }_{j} \phi_{, k}+g^{i}{ }_{k} \phi_{, j}-g_{j k} g^{i l} \phi_{, l} .
\end{aligned}
$$

The second of these equations establishes the conformal invariance of the $\Gamma^{i}{ }_{j k}$ defined by (8.5) in a new way. The first equations suggest that $\pi_{\alpha}$ in (8.6) be defined as $\eta_{\alpha}$ so that $\Gamma_{\beta \gamma}^{\alpha}$ is defined by the equations

$$
\Gamma^{\alpha}{ }_{\beta \gamma}=\left\{\begin{array}{c}
\alpha \\
\beta \gamma
\end{array}\right\}+a^{\alpha} \eta_{\gamma}+a^{\alpha}{ }_{\gamma} \eta_{\beta}-a_{\beta \gamma} a^{\alpha \delta} \eta_{\delta} .
$$

The definitions (8.5) and (8.11) show that $\Gamma^{i}{ }_{j k}$ and $\Gamma^{\alpha}{ }_{\beta \gamma}$ are of class $C^{r-3}$ in the $x^{i}$.

The quantities $\Gamma^{i}{ }_{j k}$ defined by (8.5) are called the conformal (surface) coefficients of connection and the quantities $\Gamma^{\alpha}{ }_{\beta \gamma}$ defined by (8.11) are called the conformal (space) coefficients of connection. The derivative defined by (8.1) is called the conformal (surface) derivative of $T^{\alpha i}{ }_{\beta j}$. It is clear that all the properties (a) through (e) as well as (8.4) are satisfied and that conformal differentiation of the sum, difference, inner and outer product of tensors obeys the same rules as 
ordinary differentiation. However, we note that both $\Gamma^{i}{ }_{j k}$ and $\Gamma^{\alpha}{ }_{\beta \gamma}$ are defined at points of $V_{n}$ only and consequently the conformal derivative does not have a meaning throughout the imbedding space $V_{m}$ but only on the subspace $V_{n}$. We also observe that the conformal coefficients of connection are defined relative to $V_{n}$. This means that the conformal differentiation process is dependent not only on the metric of $V_{m}$ but also on the subspace $V_{n}$ (or, more precisely, on the third order differential element of $V_{n}$ ). This definition of differentiation with respect to a subspace is somewhat analogous to the similar definition of parallel displacement of vectors in a general Riemann space with respect to a curve.

If $T^{\alpha_{\beta}}$ is a conformal tensor of class $C^{1}$ defined on a space neighborhood of $V_{m}$ which includes a surface neighborhood of $V_{n}$ then $T_{\beta: \gamma}^{\alpha_{\beta}}$ may be defined at points of the surface neighborhood as

$$
T_{\beta: \gamma}^{\alpha}=\frac{\partial}{\partial y^{\gamma}} T_{\beta}^{\alpha}+T_{\beta}^{\delta_{\beta}} \Gamma_{\delta \gamma}^{\alpha}-T_{\delta}^{\alpha_{\delta}} \Gamma_{\beta \gamma}^{\delta_{\beta \gamma}}
$$

It is immediate that this conformal (space) derivative $T^{\alpha}{ }_{\beta: \gamma}$ of $T^{\alpha}{ }_{\beta}$ enjoys all the properties of the conformal surface derivative $T_{\beta: k}^{\alpha}$. It is a simple consequence of (8.1) and (8.12) that these derivatives are related by means of the equations

$$
T_{\beta: k}^{\alpha}=T_{\beta: \gamma}^{\alpha} G^{\gamma} \text {. }
$$

According to (8.1),

$$
G_{i: j}^{\alpha}=\frac{\partial^{2} y^{\alpha}}{\partial x^{i} \partial x^{j}}+\Gamma_{\beta \gamma}^{\alpha} g_{i}^{\beta} g^{\gamma}{ }_{j}-\Gamma^{h}{ }_{i j} g^{\alpha}{ }_{h}
$$

since $G^{\alpha_{i}}=g^{\alpha}{ }_{i}$. If these equations are simplified by means of (4.5), (4.14), (5.3), (8.5) and (8.11), we obtain

$$
G^{\alpha_{i: j}}=g^{\alpha_{i j}}+g^{\alpha}{ }_{i}\left(\eta_{\gamma} g^{\gamma_{j}}-\phi_{, j}\right)+g^{\alpha}{ }_{j}\left(\eta_{\beta} g^{\beta_{i}}-\phi_{, i}\right)-g_{i j}\left(a^{\alpha \delta} \eta_{\delta}-g^{\alpha l^{l}} \phi_{, \imath}\right) .
$$

Since $\mu_{\alpha}$ is orthogonal to $g^{\alpha}{ }_{i}$, it follows from (4.14), (8.8) and (8.10) that

$$
\eta_{\beta} g^{\beta_{i}}-\phi_{, i}=0, \quad a^{\alpha \delta} \eta_{\delta}-g^{\alpha} \phi_{, l}=\mu^{\alpha} .
$$

Hence, in virtue of (6.10) and these last equations, (8.14) may be written as

$$
G^{\alpha}{ }_{i: j}=G^{\alpha}{ }_{i j} \text {. }
$$

In particular, we note as an immediate consequence of (8.15) that

$$
G^{\alpha}{ }_{i: j}=G^{\alpha}{ }_{j: i} \text {. }
$$

While the $\Gamma^{i}{ }_{j k}$ are uniquely determined by conditions (a) through (e), this is not true for the $\Gamma_{\beta \gamma}^{\alpha}$. Let $\Gamma^{\prime \alpha}{ }_{\beta \gamma}$ be any space coefficients of connection for which conditions (a) to (e) are true, other than the $\Gamma^{\alpha}{ }_{\beta \gamma}$ defined by (8.11). Then 


$$
\Gamma_{\beta \gamma}^{\prime \alpha}-\Gamma_{\beta \gamma}^{\alpha}=T_{\beta \gamma}^{\alpha},
$$

where $T^{\alpha}{ }_{\beta \gamma}$ is a tensor. It is easy to show, using conditions (a) to (e), that $T^{\alpha}{ }_{\beta \gamma}$ must be a conformal tensor which satisfies the relations

$$
T_{\alpha \beta \gamma}=T_{\alpha \gamma \beta}, \quad\left[T_{\alpha \beta \gamma}+T_{\beta \alpha \gamma}\right] G^{\gamma_{k}}=0,
$$

where $T_{\alpha \beta \gamma}=A_{\alpha \delta} T_{\beta \gamma}^{\delta}$, and conversely. It follows from (8.17) that we may write

$$
T_{\alpha \beta \gamma}+T_{\beta \alpha \gamma}=S_{\alpha \beta \gamma},
$$

where $S_{\alpha \beta \gamma}$ is any conformal tensor which obeys the equations

$$
S_{\alpha \beta \gamma}=S_{\beta \alpha \gamma}, \quad S_{\alpha \beta \gamma} G^{\gamma_{k}}=0 .
$$

From equations (8.18) and the symmetry properties of $T_{\alpha \beta \gamma}$, we easily find that

$$
T_{\alpha \beta \gamma}=\left[S_{\alpha \beta \gamma}+S_{\alpha \gamma \beta}-S_{\beta \gamma \alpha}\right] / 2 .
$$

Conversely, if $S_{\alpha \beta \gamma}$ is any conformal tensor which obeys (8.19) then $T_{\alpha \beta \gamma}$, defined by (8.20), obeys (8.17). It follows from (8.16) that the most general $\Gamma^{\prime \alpha_{\beta \gamma}}$ are given by

$$
\Gamma^{\prime \alpha_{\beta \gamma}}=\Gamma_{\beta \gamma}^{\alpha}+A^{\alpha \delta}\left[S_{\delta \beta \gamma}+S_{\delta \gamma \beta}-S_{\beta \gamma \delta}\right] / 2
$$

where $S_{\alpha \beta \gamma}$ is any conformal tensor which has the properties stated in (8.19).

Finally we note that $\Gamma^{i}{ }_{j k}$ and $\Gamma^{\alpha_{\beta \gamma}}$ exist if $r \geqq 3$. However we may show that if $\Gamma^{i}{ }_{j k}$ exists on $a V_{n}$ of class $C^{r}$ with $r \geqq 2$ then $\Gamma^{\alpha}{ }_{\beta \gamma}$ as well as the quantities $\bar{\Gamma}^{i}{ }_{j k}, \bar{\Gamma}^{\alpha}{ }_{\beta \gamma}$ defined on any $\bar{V}_{n}$ also exists. For the proof, we note that (8.5) may be solved for $\phi_{, i}$ in terms of $\Gamma^{i}{ }_{j k}$ and

$$
\left\{\begin{array}{c}
i \\
i k
\end{array}\right\} \text {. }
$$

Since $r \geqq 2, \Phi$ exists and (8.7) is true. The existence of $\phi_{, i}$ plus the validity of (8.7) permits us to deduce the existence of $\bar{\phi}_{, i}$. Hence $\Gamma^{i}{ }_{j k}$ exists. Also (8.10) and analogous equations in $\bar{V}_{n}$ show that $\eta_{\alpha}$ and $\bar{\eta}_{\alpha}$ exist. It follows from (8.6) that both $\Gamma_{\beta \gamma}^{\alpha}$ and $\Gamma_{\beta \gamma}^{\alpha}$ exist. Incidentally, the proof also shows that if $\phi_{, i}$ exists and $r \geqq 2$, then $\bar{\phi}_{, i}$ also exists.

9. The replacement principle. Many of the equations of this paper contain a mixture of some quantities which are unchanged by conformal transformations of $V_{m}$ as well as of others which vary under conformal changes of the metric of $V_{m}$. In this section, we show, by means of a change of gauge, that certain of these latter non-conformal geometric objects may be treated as independent variables in expressions where they occur and, in particular, may be replaced by zeros. This replacement principle results in a very considerable curtailment of many of the formal calculations which are made in the paper. In this respect, it is analogous to the choice of special coordinate systems (for 
example, normal coordinates or coordinates where the coordinate directions have special initial positions) frequently made in classical and Riemannian geometry.

Theorem 9.1. Let $V_{n}$ be a subspace of class $C^{r}(r \geqq 2)$ in a $V_{m}$ of class $C^{a}$ $(q \geqq r)$ and suppose that the gauge function $\phi$, its derivative $\phi_{, i}$, and the mean curvature normal $\mu_{\alpha}$ exist $\left.{ }^{24}\right)$ in $V_{n}$. Then an allowable conformal transformation of a region of $V_{m}$ containing $V_{n}$ exists so that $V_{m} \rightleftarrows \bar{V}_{m}, V_{n} \rightleftarrows \bar{V}_{n}$ and the values at any fixed point of $\bar{V}_{n}$ of the geometric quantities $\bar{\phi}, \bar{\phi}_{, i}, \bar{\mu}_{\alpha}$ defined on $\bar{V}_{n}$ are arbitrary preassigned numbers.

The proof is simple. For the existence of $\bar{\phi}, \bar{\phi}_{, i}$, and $\bar{\mu}_{\alpha}$ in $\bar{V}_{n}$ follows from the assumed existence of the corresponding quantities in $V_{n}$ and the concluding paragraph of $\S 8$. Now the values of $\bar{\phi}, \bar{\phi}_{, i}, \mu_{\alpha}$ at a point $\bar{P}$ of $\bar{V}_{n}$ are preassigned numbers and the values of $\phi, \phi_{, i}, \mu_{\alpha}$ at the corresponding point $P$ of $V_{n}$ (having the same coordinates) are known. Hence the corresponding values of $\eta_{\alpha}, \bar{\eta}_{\alpha}$ at $\Phi, \bar{\Phi}$ respectively may be computed by equations like (8.10) evaluated at these points. Then (8.7) and (8.9) determine the value of the mapping function $\sigma$ and of its first space derivative $\sigma_{, \alpha}$ at $P$. It is clear that an infinite number of functions $\sigma\left(y^{\alpha}\right)$ of class $C^{q-1}$ exist having the determined values of $\sigma, \sigma_{, \alpha}$ at $P$. Any one of these functions may serve as the conformal mapping function and satisfy the conditions of the theorem. This completes the proof.

Now consider any conformal tensor $T^{\alpha i_{\beta j}}$ defined on $V_{n}$ and depending upon geometric objects of $V_{m}$ and $V_{n}$. If $V_{m} \rightleftarrows \bar{V}_{m}, V_{n} \rightleftarrows \bar{V}_{n}$ under an allowable conformal map, we assume that the corresponding tensor $\bar{T}^{\alpha i}{ }_{\beta j}$ obeys

$$
\bar{T}^{\alpha i}{ }_{\beta j}=T^{\alpha i}{ }_{\beta j} .
$$

It is understood that $T^{\alpha i}{ }_{\beta j}$ and $\bar{T}^{\alpha i_{\beta j}}$ are the same formal functions of the corresponding geometric objects of $V_{n}, V_{m}$ and of $\bar{V}_{n}, \bar{V}_{m}$ respectively. However, despite the conformal character of $T^{\alpha i_{\beta j}}$ stated in (9.1), the formal expression for $T^{\alpha{ }^{i}}{ }_{\beta j}$ may frequently be given as a complicated mixture of conformal quantities and non-conformal quantities. In what follows, we explain how the conformal nature of $T^{\alpha i_{\beta j}}$ may be formally exhibited in an explicit manner.

If $T^{\alpha i_{\beta j}}$ depends formally upon some of the quantities $\phi, \phi_{, i}, \mu_{\alpha}$, we denote by ${ }^{0} T^{\alpha i} i_{\beta j}$ its formal value when $\phi, \phi, i, \mu_{\alpha}$ are each replaced( $\left.{ }^{25}\right)$ by zero. If $T^{\alpha i}{ }_{\beta j}$ is formally independent of these quantities, then we write ${ }^{0} T^{\alpha i}{ }_{\beta j}=T^{\alpha i}{ }_{\beta j}$. We use a similar symbolism for any (not necessarily conformal) geometric object in $V_{n}$. Whenever we speak of replacements for a geometric object in $V_{n}$, we mean the substitutions described in this paragraph.

(24) If $r \geqq 3$, all of these last conditions are fulfilled.

(25) Since zero is a tensor, these replacements leave the tensor character of $T_{\beta j}^{\alpha i}$ undisturbed. 
Let $P$ be a point of $V_{n}$. According to Theorem 9.1, a conformal map $V_{m} \rightleftarrows \bar{V}_{m}, V_{n} \rightleftarrows \bar{V}_{n}, P \rightleftarrows \bar{\Phi}$ exists so that $\bar{\phi}=\bar{\phi}_{, i}=\bar{\mu}_{\alpha}=0$ at $\bar{\Phi}$. In virtue of these equations, the value of $\bar{T}^{\alpha i}{ }_{\beta j}$ at $\overline{\mathcal{P}}$ may be written as

$$
\bar{T}^{\alpha i_{\beta j}}={ }^{0}\left(\bar{T}^{\alpha i_{\beta j}}\right) \text {. }
$$

Now if it is possible to write the formal expression for ${ }^{0}\left(T^{\alpha i}{ }_{\beta j}\right)$ so that it involves only conformal geometric objects, it remains unchanged (both formally and in its value) under conformal transformations so that

$$
{ }^{0} T^{\alpha i_{\beta j}}={ }^{0}\left(\bar{T}^{\alpha i_{\beta j}}\right),
$$

where ${ }^{0} T^{\alpha i}{ }_{\beta j}$ is evaluated at $P$. Of course, this condition is equivalent to the assumption that ${ }^{0} T^{\alpha i}{ }_{\beta j}$ is a formal function of conformal geometric objects only. A comparison of (9.1), (9.2) and (9.3) leads to

$$
T^{\alpha i}{ }_{\beta j}={ }^{0} T^{\alpha i}{ }_{\beta j},
$$

which is valid at $P$. The considerations which lead up to equations (9.1) and (9.2) may be applied at each fixed point of $V_{n}$. If (9.3) obtains throughout $V_{n}$, then equations (9.4) are valid at each point of $V_{n}$; that is, (9.4) is true throughout $V_{n}$.

The above ideas may also be applied to certain tensor equations. A tensor equation in $V_{n}$ may be written as

$$
\pi^{\alpha i}{ }_{\beta i}=0,
$$

where $\pi^{\alpha i_{\beta j}}$ is defined in terms of geometric objects of $V_{n}$ and $V_{m}$. Suppose that $\bar{\pi}^{\alpha i}{ }_{\beta j}$ is the corresponding tensor in any $\bar{V}_{n}$ defined in terms of the corresponding geometric objects of $\bar{V}_{n}, \bar{V}_{m}$ and that the equation

$$
\bar{\pi}_{\beta i}^{\alpha i}=0
$$

is obeyed in $\bar{V}_{n}$. Then it follows from (9.5) and (9.6) that $\pi^{\alpha i}{ }_{\beta j}$ is a conformal tensor $T^{\alpha i}{ }_{\beta j}$ so that (9.5) is a conformal equation. (Of course, $T^{\alpha i}{ }_{\beta j}$ is equivalent to the zero tensor.) Under these circumstances, the above discussion shows that if $T^{\alpha i}{ }_{\beta j}$ obeys (9.3), then (9.5) is equivalent to ${ }^{0} T^{\alpha i}{ }_{\beta j}=0$. We summarize these results in the replacement principle.

TheOREM 9.2. Let $T^{\alpha i_{\beta j}}\left(T^{\alpha i_{\beta j}}=0\right)$ be a conformal tensor (conformal equation) in $a V_{n}$ of class $C^{r}(r \geqq 2)$. Then, if the formal expression for ${ }^{0} T^{\alpha i}{ }_{\beta j}$ involves only conformal geometric objects, $T^{\alpha i_{\beta j}}={ }^{0} T^{\alpha i_{\beta j}}\left({ }^{0} T^{\alpha i}{ }_{\beta j}=0\right.$ is equivalent to $\left.T^{\alpha i_{j}}=0\right)$ throughout $V_{n}$.

Finally, we note that the argument used to establish this theorem says in essence: under conformal transformations, $\phi, \phi_{, i}, \mu_{\alpha}$ have the role of independent variables while $T^{\alpha i_{\beta j}}$ remains constant. Hence $T^{\alpha i}{ }_{\beta j}$ is independent of the values assigned to $\phi, \phi_{, i}, \mu_{\alpha}$ so that $T^{\alpha i}{ }_{\beta j}={ }^{0} T^{\alpha i}{ }_{\beta j}$.

It is clear that the operation of replacement is commutative with the oper- 
ations of addition, subtraction, inner and outer multiplication and projection of tensors. Whenever the operations of replacement and differentiation are both applied to a tensor, it is assumed that the replacement operation is applied last unless the contrary is indicated. For later use, we append the following equations which are immediate consequences of the definitions:

$$
\begin{array}{rlrl}
{ }^{0} \phi & ={ }^{0} \phi_{, i}={ }^{0} \mu_{\alpha}={ }^{0} \eta_{\alpha} & =0, \\
{ }^{0} g_{i j} & =G_{i j}, & { }^{0} g^{i j} & =G^{i j}, \\
{ }^{0} a_{\alpha \beta} & =A_{\alpha \beta}, & { }^{0} a^{\alpha \beta} & =A^{\alpha \beta}, \\
{ }^{0} g_{\alpha \beta} & =G_{\alpha \beta}, & { }^{0} g^{\alpha \beta} & =G^{\alpha \beta}, \\
{ }^{0}\left\{\begin{array}{c}
i \\
j_{k k}
\end{array}\right\} & =\Gamma^{i}{ }_{j k}, & \left\{\begin{array}{c}
\alpha \\
\beta \gamma
\end{array}\right\} & =\Gamma^{\alpha}{ }_{\beta \gamma}, \\
{ }^{0} \pi^{\alpha i}{ }_{\beta j, k} & =\pi^{\alpha i}{ }_{\beta j: k}, & { }^{0} \pi^{\alpha}{ }_{\beta, \gamma} & ={ }^{0} \pi^{\alpha}{ }_{\beta: \gamma} .
\end{array}
$$

Digression. The result stated in Theorem 9.1 suffices for many aspects of our work but we note that it is possible to obtain a much stronger theorem. Indeed $\phi$ and $\bar{\mu}_{\alpha}$ may be assigned as almost arbitrary functions defined not only at $\bar{\Phi}$ but throughout $\bar{V}_{n}$ if $V_{n}$ is of class $C^{r}(r \geqq 2)$ and $\phi$ is of class $C^{1}$ in $V_{n}$. To prove this, we first rearrange equations (4.3) so that the Jacobian of the first $n$ of these equations does not vanish. We denote the $y^{\alpha}$ in these equations by $y^{i}$ and the remaining $y^{\alpha}$ by $y^{\omega}(\omega=n+1, n+2, \cdots, m)$. Then equations (4.3) may be written as

$$
y^{i}=y^{i}\left(x^{j}\right), \quad y^{\omega}=y^{\omega}\left(x^{j}\right) .
$$

The parameter transformation

$$
x^{i}=y^{i}\left(x^{j}\right)
$$

is an allowable change of parameters and, in these $x^{\prime i}$, the equations of $V_{n}$ and $\bar{V}_{n}$ assume the form

$$
y^{i}=x^{i}, \quad y^{\omega}=f^{\omega}\left(x^{\prime j}\right) .
$$

We suppose that $q=r$. Then the coordinate transformation

$$
y^{\prime i}=y^{i}, \quad y^{\prime \omega}=y^{\omega}-f^{\omega}\left(x^{\prime}\right)
$$

is an allowable coordinate change. Equations (9.11) become

$$
y^{i}=x^{i}, \quad y^{\omega}=0
$$

where, for simplicity, we have omitted the primes both for the $y^{\prime \alpha}$ and the $x^{\prime i}$. From (9.12), we have

$$
g^{\alpha}{ }_{i}=\delta^{\alpha}{ }_{i}
$$

According to our hypothesis, $r \geqq 2$. Hence $\phi$ and $\mu_{\alpha}$ exist. Since we also as- 
sume the existence of $\phi_{, i}$, the vector $\eta_{\alpha}$ defined by (8.10) exists. Also, in accordance with the concluding paragraph of $\S 8$, the geometric objects $\phi_{,} \bar{\phi}_{, i}, \bar{\mu}_{\alpha}$ and $\bar{\eta}_{\alpha}$, defined on any $\bar{V}_{n}$ obtained from $V_{n}$ by an allowable conformal map must exist. Furthermore, since $\sigma$ is of class $C^{q-1}$ and $\sigma_{. \alpha}$ is of class $C^{q-2}$, it follows from (8.7) and (8.9) that $\phi-\bar{\phi}$ must be of class $C^{\alpha-1}$ and $\eta_{\alpha}-\bar{\eta}_{\alpha}$ must be of class $C^{q-2}$. According to (9.13) and the definition for $\bar{\eta}_{\alpha}$, it follows that $\mu_{\alpha}-\bar{\mu}_{\alpha}$ must be of class $C^{q-2}$.

Conversely, let $\Phi\left(x^{i}\right)$ be any scalar function and $\bar{\mu}_{\alpha}\left(x^{i}\right)$ any vector functions which satisfy the conditions:

$$
\begin{aligned}
& \phi\left(x^{i}\right)-\Phi\left(x^{2}\right) \quad \text { is of class } C^{q-1} \quad(g=r \geqq 2), \\
& \mu_{\alpha}\left(x^{i}\right)-\bar{\mu}_{\alpha}\left(x^{i}\right) \text { are of class } C^{\alpha-2}, \\
& \bar{\mu}_{\alpha} g^{\alpha_{i}}=0 .
\end{aligned}
$$

We shall show that an allowable conformal transformation $V_{m} \rightleftarrows \bar{V}_{m}, V_{n} \rightleftarrows \bar{V}_{n}$ exists so that $\Phi$ and $\bar{\mu}_{\alpha}$ are the gauge function and the mean curvature normal of $\bar{V}_{n}$.

If we define $\bar{\eta}_{\alpha}$ by the equations

$$
\bar{\eta}_{\alpha}=\bar{\mu}_{\alpha}+\phi_{, i} g^{i}{ }_{\alpha}
$$

it follows that

$$
\eta_{\alpha}-\bar{\eta}_{\alpha}=\left(\mu_{\alpha}-\bar{\mu}_{\alpha}\right)+\left(\phi_{, i}-\bar{\phi}_{, i}\right) g_{\alpha}^{i}
$$

and, from (9.14), $\eta_{\alpha}-\bar{\eta}_{\alpha}$ is of class $C^{q-2}$. As a result of (8.8), (8.10), (9.13) and (9.15), the first $n$ components $\eta_{i}$ and $\bar{\eta}_{i}$ of $\eta_{\alpha}$ and $\bar{\eta}_{\alpha}$ respectively are given by

$$
\eta_{i}=\eta_{\alpha} g^{\alpha}{ }_{i}=\phi_{, i}, \quad \bar{\eta}_{i}=\bar{\eta}_{\alpha} g_{i}^{\alpha}=\bar{\phi}_{, i} .
$$

Now, according to a theorem due to Whitney $[18$, p. 65 , Theorem 1$]$, functions $f_{\omega}\left(y^{i}, y^{\tau}\right)(\tau=n+1, n+2, \cdots, m)$ exist so that

$$
f_{\omega}\left(y^{i}, y^{\tau}\right) \text { is of class } C^{q-2} \text { in } V_{m} \text {, }
$$

$$
\begin{aligned}
& f_{\omega}\left(x^{i}, 0\right)=\eta_{\omega}\left(x^{i}\right)-\bar{\eta}_{\omega}\left(x^{i}\right), \\
& f_{\omega}\left(x^{i}, y^{\tau}\right) \text { is analytic at points of } V_{m} \text { which are not in } V_{n} .
\end{aligned}
$$

Consider the function $\sigma\left(y^{\alpha}\right)$ defined by

$$
\sigma\left(y^{\alpha}\right)=\sum_{\omega} y^{\omega} f_{\omega}\left(y^{i}, y^{\tau}\right)+\left[\phi\left(y^{i}\right)-\Phi\left(y^{i}\right)\right] .
$$

From (9.17), it follows that

$$
\sum_{\omega} y^{\omega} f_{\omega}\left(y^{i}, y^{\tau}\right)
$$

is analytic at points where at least one of the $y^{\omega}$ differs from zero and is of 
class $C^{q-2}$ at points where all the $y^{\omega}$ vanish, that is, in $V_{n}$. If $\left({ }^{26}\right) q>2$, any $(q-2)$ th partial derivative of this expression may be written in the form

$$
h\left(y^{\alpha}\right)=\sum_{\omega} y^{\omega} g_{\omega}\left(y^{i}, y^{\tau}\right)+g\left(y^{i}, y^{\tau}\right) .
$$

Here $g_{\omega}$ is a $(q-2)$ th derivative of $f_{\omega}$ and is therefore of class $C^{0}$ in $V_{n}$. Also, the highest order of derivative of the $f_{\omega}$ which occurs in $g$ is $q-3$ so that $g$ is of class $C^{1}$ in $V_{n}$.

We show that $h\left(y^{\alpha}\right)$ is of class $C^{1}$ in $V_{m}$. There are two cases: (1) If any $y^{\omega}$ is not zero, $h\left(y^{\alpha}\right)$ is analytic. (2) If all the $y^{\omega}$ vanish, then

$$
\partial h / \partial y^{j}=\partial g\left(y^{i}, 0\right) / \partial y^{j}
$$

and, by definition of the derivative,

$$
\frac{\partial h}{\partial y^{\tau}}=\lim _{\Delta y^{\tau} \rightarrow 0} \frac{1}{\Delta y^{\tau}}\left[\Delta y^{\tau} g_{\tau}\left(y^{i}, \Delta y^{\tau}\right)\right]+\frac{\partial g\left(y^{i}, 0\right)}{\partial y^{\tau}} .
$$

This last expression becomes

$$
\partial h / \partial y^{\tau}=g_{\tau}\left(y^{i}, 0\right)+\partial g\left(y^{i}, 0\right) / \partial y^{\tau} .
$$

Hence $h\left(y^{\alpha}\right)$ is of class $C^{1}$ in $V_{m}$.

Now, from (9.14), $\phi\left(y^{i}\right)-\Phi\left(y^{i}\right)$ is of class $C^{\alpha-1}$ in $V_{m}$. Hence $\sigma$, defined by (9.18), is of class $C^{q-1}$ in $V_{m}$. Furthermore,

$$
\begin{aligned}
\frac{\partial \sigma}{\partial y^{i}} & =\sum_{\omega} \frac{\partial f_{\omega}}{\partial y^{i}} \cdot y^{\omega}+\left(\frac{\partial \phi}{\partial y^{i}}-\frac{\partial \Phi}{\partial y^{i}}\right), \\
\frac{\partial \sigma}{\partial y^{\tau}} & =\sum_{\omega}\left(\frac{\partial f_{\omega}}{\partial y^{\tau}} y^{\omega}\right)+f_{\tau} .
\end{aligned}
$$

By means of (9.12), (9.16) and (9.17), we see that, at points of $V_{n},(9.18)$ becomes

$$
\begin{aligned}
\sigma & =\phi\left(x^{j}\right)-\phi\left(x^{j}\right), \\
\partial \sigma / \partial y^{i} & =\eta_{i}\left(x^{j}\right)-\bar{\eta}_{i}\left(x^{j}\right) . \\
\partial \sigma / \partial y^{\tau} & =\eta_{\tau}\left(x^{i}\right)-\bar{\eta}_{\tau}\left(x^{j}\right) .
\end{aligned}
$$

Since $\sigma\left(y^{\alpha}\right)$ is of class $C^{\ddot{q}-1}$, it defines a conformal transformation $V_{m} \rightleftarrows \bar{V}_{m}$, $V_{n} \rightleftarrows \bar{V}_{n}$ so that points with the same coordinates and parameters correspond. Then a comparison of (9.20) with (8.7) and (8.9) shows that $\phi$ is the gauge function of $\bar{V}_{n}$ and that $\bar{\mu}_{\alpha}$ is the mean curvature normal of $\bar{V}_{n}$. This completes the proof of the above italicized statement.

As a special case of this result, we note that we may choose $\phi=\phi, \bar{\mu}_{\alpha}=0$. Since $\mu_{\alpha}$ is of class $C^{r-2}$, conditions (9.14) and (9.15) are satisfied. Hence a $\bar{V}_{n}$

(2) If $q=2$, then $h\left(y^{\alpha}\right)=\sum_{\omega} y^{\omega} f_{\omega}\left(y^{i}, y^{r}\right)$ in the following argument. 
exists for which $\bar{\mu}_{\alpha}=0$. Hence any $V_{n}$ of class $C^{q}$ is conformal to a minimal subspace. However this proof requires that $n$ exceed 1 . Actually, the conclusion may be extended to apply to the case $n=1$. We state this result in the theorem.

Theorem 9.3. A subspace $V_{n}$ of class $C^{a}$ in a $V_{m}$ of class $C^{a}(q \geqq 2)$ is conformally equivalent to a subspace of zero mean curvature (minimal subspace if $n>1$, geodesic curve if $n=1$ ).

For the proof, we simply note that the theorem of Whitney used above may be employed to show the existence of functions $f\left(y^{i}, y^{\tau}\right)$ with the properties enumerated in (9.17) except that the second set of equations is replaced by

$$
f_{\omega}\left(y^{i}, 0\right)=\mu_{\omega} .
$$

Then, as above, the function $\sigma\left(y^{\alpha}\right)$ defined by

$$
\sigma\left(y^{\alpha}\right)=\sum_{\omega} y^{\omega} f_{\omega}\left(y^{i}, y^{\tau}\right)
$$

is of class $C^{q-1}$ in $V_{m}$. Also, at points of $V_{n}$,

$$
\partial \sigma / \partial y^{i}=0, \quad \partial \sigma / \partial y^{\tau}=\mu_{\tau} .
$$

Now, $\mu_{\alpha}$ is orthogonal to $g^{\alpha}{ }_{i}$. Hence, from (9.13), the first $n$ components $\mu_{i}$ of $\mu_{\alpha}$, defined by $\mu_{i}=\mu_{\alpha} \delta^{\alpha}{ }_{i}$, must be zero. Therefore the above equations become

$$
\partial \sigma / \partial y^{\alpha}=\mu_{\alpha}
$$

at points of $V_{n}$. If $\sigma\left(y^{\alpha}\right)$ is used as a conformal mapping function, it follows from (6.8) that $\bar{\mu}_{\alpha}$ vanishes identically. This completes the proof. Theorem 9.3, with $n$ equal to 1 , was used in a previous paper $[8, \S 10]$.

10. Conformal geodesic and conformal normal coordinates and parameters. When the coordinates $y^{\alpha}$ (parameters $x^{i}$ ) are subjected to an allowable transformation (that is, with $r \geqq 3$ ), the $\Gamma_{\beta \gamma}^{\alpha}\left(\Gamma^{i}{ }_{j k}\right)$ change according to the law of transformation of coefficients of connection. If primes are used to denote the new coordinate (parameter) system and the components of the $\Gamma^{\alpha}{ }_{\beta \gamma}, \Gamma^{i}{ }_{j k}$ in this system, then this law of transformation is

$$
\begin{aligned}
& \Gamma_{\beta \gamma}^{\prime \delta} \frac{\partial y^{\alpha}}{\partial y^{\prime \delta}}=\Gamma_{\delta \epsilon}^{\alpha} \frac{\partial y^{\delta}}{\partial y^{\prime \beta}} \frac{\partial y^{\epsilon}}{\partial y^{\prime \gamma}}+\frac{\partial^{2} y^{\alpha}}{\partial y^{\prime \beta} \partial y^{\prime \gamma}}, \\
& \Gamma^{\prime h}{ }_{j k} \frac{\partial x^{i}}{\partial x^{\prime h}}=\Gamma_{h l}^{i} \frac{\partial x^{h}}{\partial x^{\prime j}} \frac{\partial x^{l}}{\partial x^{\prime k}}+\frac{\partial^{2} x^{i}}{\partial x^{\prime j} \partial x^{\prime k}} .
\end{aligned}
$$

It is clear from these equations that allowable transformations of $y^{\alpha}, x^{i}$ exist $\left({ }^{27}\right)$ so that

$\left.{ }^{(27}\right)$ For example, the equations $y^{\prime \alpha}=\left(y^{\alpha}\right)_{0}+y^{\prime \alpha}-\left(\Gamma_{\beta \gamma}^{\alpha}\right)_{0} y^{\prime \beta} y^{\prime \gamma} / 2$, where $\left(y^{\alpha}\right)_{0},\left(\Gamma_{\beta \gamma}^{\alpha}\right)_{0}$ are the values of $y^{\alpha}, \Gamma_{\beta \gamma}^{\alpha}$ at $\mathcal{P}$, define a coordinate transformation for which (10.2) is true. 


$$
\begin{aligned}
\left(\Gamma^{\prime \alpha}{ }_{\beta \gamma}\right)_{0} & =0, \\
\left(\Gamma^{\prime \prime}{ }_{j k}\right)_{0} & =0
\end{aligned}
$$

at any preassigned point $\mathcal{P}$ of $V$. The new coordinate system $\left\{y^{\prime \alpha}\right\}$ [parameter system $\left.\left\{x^{\prime i}\right\}\right]$ in which $(10.2)[(10.3)]$ is true is called a coordinate [parameter] system which is conformal geodesic at $P$. These coordinates are analogous to the geodesic coordinates of Riemannian geometry.

It is also possible to define parameters $\left({ }^{28}\right)$ which are conformal normal at $P$. These are similar to the normal coordinates of Riemannian geometry. Parameters $x^{\prime i}$ which are conformal normal at $P$ are parameters so that the equations of those "geodesics" based upon the tensor $G_{i j}$ which pass through $P$ are $x^{\prime i}=L^{i} S$ where the $L^{i}$ are constants and $S$ is the conformal length based upon the tensor $G_{i j}$. Of course, conformal normal parameters are also conformal geodesic at $P$. It is sometimes useful to choose the parametric directions at the center $\boldsymbol{P}$ of the conformal normal parameters so that they coincide with $n$ mutually orthogonal curvature directions of $V_{n}$ at $P$.

The existence of conformal normal parameters is established by exactly the same proof as that used in Riemannian geometry. For this existence proof, it is sufficient that $G_{i j}$ be of class $C^{2}$ in the $x^{i}$, or otherwise, that $r \geqq 4$. The parameter transformation to conformal normal parameters is, in general, of class $C^{r-2}$ and therefore not an allowable transformation unless $r=\infty$ or unless $V_{n}$ is considered as a space of class $C^{r-2}$.

11. The Weyl tensors. The assumption

$$
g \geqq 3
$$

guarantees the existence of the Riemann curvature tensors $\rho_{\alpha \beta \gamma \delta}$ and $\bar{\rho}_{\alpha \beta \gamma \delta}$ of $V_{m}$ and $\bar{V}_{m}$ respectively. A straightforward calculation based on the definition (5.14) and the first equations of (6.3) gives $\left({ }^{29}\right)$

$$
e^{-2 \sigma} \bar{\rho}_{\alpha \beta \gamma \delta}=\rho_{\alpha \beta \gamma \delta}+a_{\alpha(\delta} \sigma_{\gamma)-\beta}+a_{\beta(\gamma} \sigma_{\delta)-\alpha}+a_{\alpha(\delta} a_{\gamma)-\beta} \cdot \Delta_{1} \sigma .
$$

From these equations, we readily obtain

$$
\bar{\rho}_{\beta \gamma}=\rho_{\beta \gamma}+(m-2) \sigma_{\beta \gamma}+a_{\beta \gamma}\left[a^{\alpha \delta} \sigma_{, \alpha \delta}+(m-2) \Delta_{1} \sigma\right]
$$

and

$$
\begin{aligned}
(m-1)(m-2) \sigma_{\alpha \beta}= & (m-1)\left[\bar{\rho}_{\alpha \beta}-\rho_{\alpha \beta}\right]-\left[\bar{\rho} \bar{a}_{\alpha \beta}-\rho a_{\alpha \beta}\right] / 2 \\
& -(m-1)(m-2) \Delta_{1} \sigma \cdot a_{\alpha \beta} / 2 .
\end{aligned}
$$

In these equations, $\Delta_{1} \sigma$ is the differential parameter of the first order defined by $\Delta_{1} \sigma=a^{\alpha \beta} \sigma_{, \alpha} \sigma_{, \beta}$ and $\sigma_{\alpha \beta}$ is defined by the equations

$$
\sigma_{\alpha \beta}=\sigma_{, \alpha \beta}-\sigma_{, \alpha} \sigma_{, \beta} .
$$

(28) Since the $\Gamma_{\beta \gamma}^{\alpha}$ are only defined at points of $V_{n}$, there are obvious difficulties in defining "conformal normal coordinates."

(29) For a derivation of (11.2) along these lines, see [4, pp. 89-90]. 
If $m>2$, (11.3) may be solved for $\sigma_{\alpha \beta}$. If the resulting value for $\sigma_{\alpha \beta}$ is substituted in (11.2) and account is taken of (2.6), we find that

$$
\bar{C}^{\alpha}{ }_{\beta \gamma \delta}=C^{\alpha}{ }_{\beta \gamma \delta}
$$

where $C^{\alpha}{ }_{\beta \gamma \delta}$ is the conformal (space) Weyl tensor of $V_{m}(m>2)$ defined by

$$
C_{\beta \gamma \delta}^{\alpha}=\rho^{\alpha}{ }_{\beta \gamma \delta}+\frac{1}{(m-2)}\left[a^{\alpha}{ }_{(\gamma \rho \delta)-\beta}+a_{\beta\left(\delta \rho^{\alpha} \gamma\right)-}\right]
$$

$$
+\frac{\rho}{(m-1)(m-2)} \cdot a^{\alpha}{ }_{(\delta} a_{\gamma)-\beta} .
$$

If $m=3$, it may be shown that $C^{\alpha}{ }_{\beta \gamma \delta}$ is a zero tensor. It was proved by Weyl $\left[17\right.$, p. 404] and Schouten $\left[12\right.$, p. 80] that, if $m>3$, the equation $C^{\alpha}{ }_{\beta \gamma \delta}=0$ is the necessary and sufficient condition that $V_{m}$ be a conformally euclidean space $\bar{R}_{m}$.

Unlike the Riemann tensor, contraction of indices in the Weyl tensor does not lead to any new tensors. For, from (11.5),

$$
C_{\beta \gamma \alpha}^{\alpha}=C_{\beta \alpha \delta}^{\alpha}=C_{\alpha \gamma \delta}^{\alpha}=C_{\beta \gamma \delta}^{\alpha} a^{\beta \gamma}=C_{\beta \gamma \delta}^{\alpha} a^{\beta \delta}=C_{\beta \gamma \delta}^{\alpha} a^{\gamma \delta}=0 .
$$

However, new conformal tensors may be constructed from $C^{\alpha}{ }_{\beta \gamma \delta}$ at points of $V_{n}$ by means of the conformal projection tensor $G^{\alpha \beta}$ and the indices of these various tensors may be raised or lowered by means of $A^{\alpha \beta}, A_{\alpha \beta}$ if (5.1) holds. We define the following conformal tensors:

$$
\begin{array}{rlrl}
C_{\alpha \beta \gamma \delta} & =A_{\alpha \epsilon} C^{\boldsymbol{\epsilon}_{\beta \gamma \delta},} \\
C_{\beta \gamma} & =C_{\alpha \beta \gamma \delta} G^{\alpha \delta}, \\
C & =C_{\beta \gamma} G^{\beta \gamma}, \\
C_{\alpha \beta \gamma k} & =C_{\alpha \beta \gamma \delta} G^{\delta}{ }_{k}, & \\
C_{\alpha i j k} & =C_{\alpha \beta j k} G^{\beta}, & C_{\alpha \beta i k}=C_{\alpha \beta \gamma k} G^{\gamma}, & C_{h i j k}=C_{\alpha i j k} G^{\alpha}, \\
C_{\beta j} & =C_{\beta \gamma} G^{\gamma}, \quad & C_{i j}=C_{\beta j} G^{\beta}{ }_{i} .
\end{array}
$$

It follows easily that

$$
C_{i j}=C_{h i j k} G^{h k}, \quad C=C_{i j} G^{i j} .
$$

According to (11.6), (11.7) and (11.8),

$$
\begin{aligned}
C_{\alpha \beta \gamma \delta} A^{\alpha \beta}=C_{\alpha \beta \gamma \delta} A^{\alpha \gamma}=C_{\alpha \beta \gamma \delta} A^{\alpha \delta}=C_{\alpha \beta \gamma \delta} A^{\beta \gamma}=C_{\alpha \beta \gamma \delta} A^{\beta \delta}=C_{\alpha \beta \gamma \delta} A^{\gamma \delta}=0, \\
C_{\alpha \beta} A^{\alpha \beta}=0 .
\end{aligned}
$$

As an easy consequence of (5.15), (11.5) and (11.7), we have the identities

$$
C_{\alpha \beta \gamma \delta}=C_{\gamma \delta \alpha \beta}=-C_{\beta \alpha \gamma \delta}=-C_{\alpha \beta \delta \gamma}, \quad C_{\alpha(\beta \gamma \delta)^{+}}=0 .
$$

Similar identities aiso hold for $C_{h i j k}$. From these last equations, we also obtain 


$$
\begin{aligned}
C_{\alpha \beta \gamma \delta} G^{\alpha \beta} & =C_{\alpha \beta \gamma \delta} G^{\gamma \delta}=0, \\
C_{\beta \gamma} & =C_{\beta \alpha \delta \gamma} G^{\alpha \delta}, \\
C_{\beta \gamma} & =C_{\gamma \beta} .
\end{aligned}
$$

The conformal tensors $D^{\alpha}{ }_{\beta j k}$ and $D^{\alpha_{i j k}}$ which appear in later sections of the paper are defined by the equations

$$
\begin{aligned}
& \left.D_{\beta j k}^{\alpha_{\beta j}}=\left\{C_{\beta \gamma \delta}^{\alpha_{\beta \gamma}}+\frac{1}{(n-1)}\left[A^{\alpha}{ }_{(\gamma} C_{\delta)-{ }_{\epsilon} H^{\epsilon}{ }_{\beta}}+A_{\beta(\delta} C_{\gamma}^{\epsilon}\right)^{H_{\epsilon}} H^{\alpha}\right]\right\} G^{\gamma}{ }_{j} G^{\delta_{k}}, \\
& D_{\alpha \beta j k}=A_{\alpha \gamma} D^{\gamma}{ }_{\beta j k} \text {, } \\
& \left.D^{\alpha_{i j k}}=C^{\alpha_{i j k}}+\frac{1}{(n-1)} H^{\alpha}{ }_{e} C_{\gamma} G_{i(k} G^{\gamma}{ }^{\gamma}\right)^{-}, \\
& D_{\alpha i j k}=A_{\alpha \gamma} D^{r_{i j k}},
\end{aligned}
$$

where the raising of indices in $C_{\alpha \beta}$ is naturally accomplished by means of $A^{\alpha \beta}$. It is clear that

$$
D^{\alpha_{i j k}}=D^{\alpha}{ }_{\beta j k} G_{i}^{\beta}, \quad D_{\alpha i j k}=D_{\alpha \beta j k} G_{i}^{\beta} .
$$

If $m=n+1$, so that $V_{n}$ is a hypersurface, the measure tensor $A^{\alpha \beta}$ may be written as

$$
A^{\alpha \beta}=G^{\alpha \beta}+Z^{\alpha} Z^{\beta},
$$

where $Z^{\alpha}$ is the unit conformal normal to $V_{n}$ (determined except for algebraic sign) so that

$$
A_{\alpha \beta} Z^{\alpha} G^{\beta}{ }_{j}=0, \quad A_{\alpha \beta} Z^{\alpha} Z^{\beta}=1 .
$$

It follows from (11.8), (11.13) and (11.20) that

$$
C_{\beta \gamma}=-C_{\alpha \beta \gamma \delta} Z^{\alpha} Z^{\delta} \text {. }
$$

As a consequence of (11.9), (11.13), (11.14), (11.20) and (11.22), the following identities also hold for a hypersurface

$$
C=C_{\alpha \beta \gamma \delta} Z^{\alpha} Z^{\beta} Z^{\gamma} Z^{\delta}=0, \quad C_{\beta \gamma} Z^{\beta}=0 .
$$

The last set of equations shows that $C_{\beta \gamma}$ is a tangent conformal tensor. Finally we note that, for a hypersurface, the conformal tensors $D^{\alpha_{\beta j k}}$ and $D^{\alpha_{i j k}}$, defined by (11.18) and (11.19), become

$$
D^{\alpha_{\beta j k}}=C^{\alpha_{\beta j k}}, \quad D^{\alpha_{i j k}}=C^{\alpha_{i j k}} .
$$

If (11.1) is replaced by (5.13), the Riemann curvature tensors $\kappa_{i j k l}$ and $\bar{\kappa}_{i j k l}$ of $V_{n}$ and $\bar{V}_{n}$ respectively exist. As in the beginning of this section, we may construct the conformal (surface) Weyl tensors of $V_{n}$ and $\bar{V}_{n}$ from the Riemann tensors if $n>2$. The Weyl tensor $W^{i}{ }_{j k l}$ of $V_{n}(n>2)$ is defined by 


$$
\begin{aligned}
& W^{i}{ }_{j k l}=\kappa^{i}{ }_{j k l}+\frac{1}{(n-2)}\left[g^{i}{ }_{(k k)^{-}{ }^{j}}+g_{j\left(l k^{i}{ }^{i}\right)^{-}}\right] \\
& +\frac{\kappa}{(n-1)(n-2)} \cdot g^{i}\left({ }^{\prime} g_{k)}-j .\right.
\end{aligned}
$$

If $n=3, W^{i}{ }_{j k l}$ is a zero tensor. If $n$ exceeds 3 , the vanishing of $W^{i}{ }_{j k l}$ is the necessary and sufficient condition that $V_{n}$ be a conformally euclidean space. The indices of $W^{i}{ }_{j k l}$ may be raised or lowered by means of $G^{i j}, G_{i j}$ without changing its conformal character. For example,

$$
W_{i j k l}=G_{i h} W^{h}{ }_{j k l} \text {. }
$$

Since equations like (11.13) hold when $W_{i j k l}, G^{i j}$ replace $C_{\alpha \beta \gamma \delta}, A^{\alpha \beta}$ there are no analogues to $C_{\alpha \beta}, C$. It is easy to verify that the usual identities hold for $W_{i j k l}$, that is,

$$
W_{i j k l}=W_{k l i j}=-W_{j i k l}=-W_{i j l k}, \quad W_{i(j k l)^{+}}=0 .
$$

We now define the Weyl operation $\uparrow$ which when applied to $\rho^{\alpha}{ }_{\beta \gamma \delta}, \kappa^{i}{ }_{j k l}$ yields the Weyl tensors $C^{\alpha}{ }_{\beta \gamma \delta}, W^{i}{ }_{j k l}$. This operation is used later in the paper. Let $n>2$ and $f_{i j}$ be any symmetric positive definite surface tensor and let $d_{i j k l}\left(d^{i}{ }_{j k l}\right)$.be any surface tensor. Then the Weyl $\uparrow$ operation with respect to $f_{i j}$ applied to the tensor $d_{i j k l}\left(d^{i}{ }_{j k l}\right)$ yields the tensor $\uparrow d_{i j k l}\left(\uparrow d^{i}{ }_{j k l}\right)$ defined by the equations

$$
\begin{aligned}
\uparrow d_{i j k l}=d_{i j k l} & +\frac{1}{(n-2)}\left[f_{i(k} d_{l)^{-} j}+f_{j(l)} d_{k)-i}\right] \\
& +\frac{d}{(n-1)(n-2)} f_{i(l)} f_{k)^{-} j}, \\
\uparrow d^{i}{ }_{j k l}=d^{i}{ }_{j k l} & +\frac{1}{(n-2)}\left[\delta^{i}{ }_{(k} d_{l)-j}+f_{j(l)} d^{i}{ }_{k)-}\right] \\
& +\frac{d}{(n-1)(n-2)} \delta^{i}{ }_{(l)} f_{k)-j},
\end{aligned}
$$

where $f^{i h}$ are the contravariant components of $f_{i h}$ and $d_{i l}=d_{i j k l} f{ }^{j k}, d^{i}{ }_{l}=d^{i}{ }_{j k l} f^{j k}$, $d=d_{i l} f^{i l}=d^{i}{ }_{i}$. A similar definition is made for space tensors. Then it is immediate that

$$
C^{\alpha}{ }_{\beta \gamma \delta}=\uparrow \rho^{\alpha}{ }_{\beta \gamma \delta}, \quad W^{i}{ }_{j k l}=\uparrow \kappa^{i}{ }_{j k l}
$$

where the $\uparrow$ operations in these equations are with respect to $a_{\alpha \beta}$ and $g_{i j}$ respectively. The following rules are direct consequences of the above definitions:

$$
\uparrow\left(d_{i j k l}+e_{i j k l}\right)=\uparrow d_{i j k l}+\uparrow e_{i j k l}
$$




$$
\begin{gathered}
\uparrow\left(f_{i\left(k l_{l}\right)^{-}}+f_{j\left(l \left(e_{k)-i}\right.\right.}\right)=0, \\
f^{j k} \uparrow d_{i j k l}=0 .
\end{gathered}
$$

As a consequence of the last equations,

$$
\uparrow \uparrow d_{i j k l}=\uparrow d_{i j k l} \text {. }
$$

12. The Bianchi identities for the Weyl tensors. If (10.1) is replaced by the stronger inequality

$$
g \geqq 4,
$$

the covariant derivative of the space Riemann tensor $\rho^{\alpha}{ }_{\beta \gamma \delta}$ exists. If we choose geodesic coordinates at a point $\mathcal{P}$ of $V_{m}$, differentiation of (5.14) and evaluation at $\mathcal{P}$ yields

$$
\rho_{\beta \gamma \delta, \epsilon}^{\alpha}=\frac{\partial^{2}}{\partial y^{\epsilon} \partial y^{\gamma}}\left\{\begin{array}{c}
\alpha \\
\beta \delta
\end{array}\right\}-\frac{\partial^{2}}{\partial y^{\epsilon} \partial y^{\delta}}\left\{\begin{array}{c}
\alpha \\
\beta \gamma
\end{array}\right\} .
$$

From this result and similar equations, we obtain $[4$, p. 82]

$$
\rho_{\beta(\gamma \delta, e)+}^{\alpha}=0
$$

at $\boldsymbol{P}$. Since the terms in these equations are the components of a tensor, the equations hold in all coordinate systems and at all points of $V_{m}$. The equations are the weil known Bianchi identities. If (12.2) is contracted for $\alpha$ and $\delta$ and also multiplied by $a^{\beta \epsilon}$ and summed for $\beta, \epsilon$, we obtain

$$
a^{\beta \epsilon} \rho_{\beta \gamma, \epsilon}=\rho, \gamma / 2
$$

after using (5.15) and the definitions for $\rho_{\beta \gamma}$ and $\rho$.

If $m>2$ and $C^{\alpha}{ }_{\beta \gamma \delta, \epsilon}$ is calculated from (11.5), we obtain, after using (12.2),

$$
C_{\beta(\gamma \delta, \epsilon)^{+}}^{\alpha}=\frac{1}{(m-2)}\left[a_{\gamma^{\alpha} \rho_{\beta(\delta \epsilon)+}+}+a_{\beta \delta+\rho^{\alpha}(\gamma \epsilon)^{+}}\right]
$$

where we have written

$$
\rho_{\beta \gamma \delta}=\rho_{\beta(\gamma, \delta)}+\frac{1}{2(m-1)} \cdot \rho_{,(\gamma} a_{\delta)-\beta}, \quad \rho^{\alpha}{ }_{\gamma \delta}=a^{\alpha \beta} \rho_{\beta \gamma \delta} .
$$

It follows from (12.3) and (12.5) that

$$
\rho^{\alpha}{ }_{\alpha \delta}=0 .
$$

Contraction of (12.4) for $\alpha$ and $\delta$ leads to

$$
C_{\beta \epsilon \gamma, \alpha}^{\alpha}=(m-3) \rho_{\beta \epsilon \gamma} /(m-2)
$$

after simplification by means of (11.6) and (12.6).

Since $C^{\alpha}{ }_{\beta \gamma \delta}$ is of class $C^{1}$ throughout $V_{m}, C^{\alpha}{ }_{\beta \gamma \delta: \epsilon}$ exists at points of $V_{n}$ if condition (5.13) is adjoined to (12.1). From (8.11), (8.12), (11.6) and (11.14), we obtain 


$$
C_{\beta \gamma \delta: \epsilon}^{\alpha}=C_{\beta \gamma \delta, \epsilon}^{\alpha}+(m-3) C_{\beta \gamma \delta}^{\alpha} \eta_{\alpha} .
$$

This equation is true on $V_{n}$. It follows from (12.7) and (12.8) that, at points of $V_{n}$

$$
C^{\alpha}{ }_{\beta \gamma \delta: \alpha}=(m-3)\left[\frac{1}{(m-2)} \rho_{\beta \gamma \delta}+C^{\alpha}{ }_{\beta \gamma \delta} \eta_{\alpha}\right] .
$$

According to (9.10),

$$
{ }^{\alpha} C_{\beta \gamma \delta, \epsilon}^{\alpha}=C^{\alpha}{ }_{\beta \gamma \delta: є \cdot}
$$

If $m>3,(9.7)$ and (12.9) show that

$$
{ }^{0} \rho_{\beta \gamma \delta}=(m-2) C{ }_{\beta \gamma \delta: \alpha} /(m-3) .
$$

Now equations analogous to (12.4) are true in every $\bar{V}_{m}$ so that (12.4) are conformal equations. If replacements are made for the various tensors in these equations by means of (9.8), (12.10) and (12.11), we obtain

$$
C_{\beta(\gamma \delta: \epsilon)^{+}}^{\alpha}=\frac{1}{(m-3)}\left[A^{\alpha}{ }_{\gamma^{+}+C_{\beta(\delta \epsilon)+: \zeta}}+A_{\beta \delta+C^{\zeta \alpha}(\gamma \epsilon)^{+} \zeta \zeta}\right]
$$

where $C^{\zeta \alpha}{ }_{\gamma \epsilon}=C_{\beta \gamma \epsilon}^{\gamma^{\alpha}} A^{\alpha \beta}$. Since the terms in (12.12) are components of conformal tensors, the replacement principle enables us to conclude that (12.12) is equivalent to (12.4) at points of $V_{n}$. Consequently equations (12.12) are valid throughout $V_{n}$ if $m>3$. They are the Bianchi identities for the space Weyl tensor $C^{\alpha}{ }_{\beta \gamma \delta}$.

If $m \leqq 3$, no corresponding Bianchi identities for $C^{\alpha}{ }_{\beta \gamma \delta}$ hold since $C^{\alpha}{ }_{\beta \gamma \delta}$ does not exist if $m=2$ and is a zero tensor if $m=3$. If $m=2, \rho_{\alpha \beta}=\rho \cdot a_{\alpha \beta} / 2$ so that $\rho_{\alpha \beta \gamma}$ defined by (12.5) is a zero tensor. If $m>2$, covariant differentiation of (11.3) and use of (2.6) and (6.3) gives

$$
\begin{aligned}
& (m-2)\left[\sigma_{, \alpha \beta \gamma}-\sigma_{, \alpha \gamma} \sigma_{, \beta}-\sigma_{, \alpha} \sigma_{, \beta \gamma}\right]=\bar{\rho}_{\alpha \beta ; \gamma}+2 \bar{\rho}_{\alpha \beta} \sigma_{, \gamma} \\
& \quad+\bar{\rho}_{\gamma \beta} \sigma_{, \alpha}+\bar{\rho}_{\alpha \gamma} \sigma_{, \beta}-\bar{\rho}_{\epsilon \beta} a^{\epsilon \delta} \sigma_{, \delta} a_{\alpha \gamma}-\bar{\rho}_{\epsilon \alpha} a^{\epsilon \delta} \sigma_{, \delta} a_{\beta \gamma} \\
& \quad-\rho_{\alpha \beta, \gamma}-(1 / 2(m-1))\left[\bar{\rho}_{; \gamma} \bar{a}_{\alpha \beta}+2 \bar{\rho} \bar{a}_{\alpha \beta} \sigma_{, \gamma}-\rho_{, \gamma} a_{\alpha \beta}\right] \\
& \quad-(m-2) a_{\alpha \beta} a^{\epsilon \delta} \sigma_{, \epsilon \gamma} \sigma_{, \delta} .
\end{aligned}
$$

According to the Ricci identities $\left({ }^{30}\right)$ for this type of differentiation, $\sigma_{, \alpha(\beta \gamma)^{-}}=\sigma_{, \epsilon} \rho_{\alpha \beta \gamma}{ }_{\alpha \beta}$ which, if $m>2$, become

$$
\begin{aligned}
\sigma_{, \alpha(\beta \gamma)^{-}}=\sigma_{, \epsilon} C_{\alpha \beta \gamma \gamma} & +(1 /(m-2))\left[\sigma_{,(\gamma} \rho_{\beta}\right)_{-\alpha}+a_{\left.\alpha\left(\beta \rho_{\gamma}\right)_{-\epsilon} a^{e \delta} \sigma_{, \delta}\right]} \\
& +(\rho /(m-1)(m-2)) \sigma_{,(\beta} a_{\gamma)^{-\alpha}}
\end{aligned}
$$

upon application of (11.5).

(30) These identities are derived for general covariant differentiation in $\$ 17$. 
If the indices $\beta, \gamma$ in (12:13) are interchanged and the new equations subtracted from (12.13), the resulting equations may be simplified by means of (11.3), (11.4), (12.5) and (12.14). In this manner, we obtain

$$
(m-2) \sigma_{, \ell} C^{e_{\alpha \beta \gamma}}=\bar{\rho}_{\alpha \beta \gamma}-\rho_{\alpha \beta \gamma} .
$$

According to (8.9), (12.15) is equivalent to

$$
\rho_{\alpha \beta \gamma}+(m-2) \eta_{\epsilon} C_{\alpha \beta \gamma}^{\boldsymbol{\epsilon}_{\alpha \beta}}=\bar{\rho}_{\alpha \beta \gamma}+(m-2) \bar{\eta}_{\epsilon} \bar{C}_{\alpha \beta \gamma} .
$$

If $m=3$ or if $V_{m}$ is a conformally euclidean space $\bar{R}_{m}$ of any dimensionality, $C^{{ }^{\alpha}{ }_{\alpha \beta \gamma}} \equiv 0$. Consequently, from (12.15), $\rho_{\alpha \beta \gamma}$ is a conformal tensor in an $R_{m}$ $(m \geqq 3)$ and in any $V_{3}$. Schouten $[12$, p. 80$]$ proved that $\rho_{\alpha \beta \gamma}$ is a zero tensor in a $V_{3}$ if and only if $V_{3}$ is an $\bar{R}_{3}$. According to (12.9) and (12.16), if $m>2$, the tensor $\rho_{\alpha \beta \gamma}+(m-2) \eta_{c} C_{\alpha \beta \gamma}$ is a conformal tensor at all points of $a V_{n}$ in $V_{m}$. It is equal to $(m-2) C^{*}{ }_{\alpha \beta \gamma:}: /(m-3)$ if $m>3$.

According to (4.13), the surface components $C_{h i j k}$ of the projection of $C_{\alpha \beta \gamma \delta}$ in the tangent vector space of $V_{n}$ are given by (11.10). Conformal differentiation of these equations with respect to $x^{\imath}$ gives

$$
\begin{aligned}
& C_{h i j k: \imath}=C_{\alpha \beta \gamma \delta: e} G^{\alpha}{ }_{h} G^{\beta}{ }_{i} G^{\gamma}{ }_{j} G^{\delta_{k}} G^{e_{\imath}} \\
& +C_{\alpha \beta \gamma \delta}\left[G^{\alpha}{ }_{h l} G^{\beta}{ }_{i} G^{\gamma}{ }_{j} G^{\delta}{ }_{k}+G^{\alpha}{ }_{h} G^{\beta}{ }_{i} G^{\gamma}{ }_{j} G^{\delta}{ }_{k}+G^{\alpha}{ }_{h} G^{\beta}{ }_{i} G^{\gamma}{ }_{j l} G^{\delta}{ }_{k}+G^{\alpha}{ }_{h} G^{\beta}{ }_{i} G^{\gamma}{ }_{j} G^{\delta}{ }_{k l}\right]
\end{aligned}
$$

after account is taken of (8.13) and (8.15). If these equations and similar equations are added and the result is simplified by means of (11.14) and (12.12), we obtain

$$
\begin{aligned}
& C_{h i(j k: l)^{+}}=\frac{1}{(m-3)}\left[G_{h j+} C^{\alpha}{ }_{i(k l)+: \alpha}+G_{i k+} C^{\alpha}{ }_{h(j l)+: \alpha}\right] \\
& +C_{\alpha \beta \gamma \delta}\left[G^{\alpha}{ }_{h j}+G^{\beta}{ }_{i} G^{\gamma}{ }_{k+} G^{\delta}{ }_{l^{+}}+G^{\alpha}{ }_{h} G^{\beta}{ }_{i j}+G^{\gamma}{ }_{k+} G^{\delta}{ }_{l^{+}}\right]
\end{aligned}
$$

where( $\left.{ }^{31}\right) C^{\alpha}{ }_{i j k: \alpha}$ are the surface components of the projection of $C^{\alpha}{ }_{\beta \gamma \delta: \alpha}$ in the tangent vector space of $V_{n}$, that is,

$$
C^{\alpha_{i j k: \alpha}}=C^{\alpha}{ }_{\beta \gamma \delta: \alpha} G^{\beta}{ }_{i} G^{\gamma}{ }_{j} G^{\delta} .
$$

We multiply (12.17) by $G^{h l}$ and sum on repeated indices and simplify the result by means of (7.4), (7.10), (7.11), (11.8), (11.11) and (11.14). In this way we obtain

$$
\begin{aligned}
C_{h i(j k: l)+G^{h l}=} & (1 /(m-3))\left[(n-3) C^{\alpha}{ }_{i j k: \alpha}+G_{i j}-C^{\alpha}{ }_{h l k^{-}: \alpha} G^{h l}\right] \\
& +C_{\alpha \beta \gamma \delta} G^{h l}\left[G^{\alpha}{ }_{h j}-G^{\beta}{ }_{i} G^{\gamma}{ }_{k}-G^{\delta}{ }_{l}+G^{\alpha}{ }_{h} G^{\beta}{ }_{i} G^{\gamma}{ }_{j} G^{\delta}{ }_{k}\right]+C_{\beta(k} G^{\beta}{ }_{j)^{-i}}
\end{aligned}
$$

In a similar manner, conformal differentiation of $C_{\beta j}$ defined by (11.11)

(31) In accordance with the convention of $\$ 4$, we omit the projection symbol $*$ since $C^{\alpha}{ }_{i i k: \alpha}$ can only mean $\left(C^{\alpha}{ }_{\beta \gamma \delta: \alpha}\right) G^{\beta}{ }_{i} G^{\gamma}{ }_{j} G^{\delta}{ }_{k}$. For the other interpretation, namely $\left(C^{\alpha}{ }_{i j k}\right): \alpha$, is meaningless since the space conformal derivative of $C^{\alpha}{ }_{i j k}$ does not exist. These remarks also apply to similar tensors defined in this section. 
with respect to $x^{k}$ gives

$$
C_{\alpha j: k}=C_{\alpha \beta \gamma \delta: c} G^{\beta \gamma} G^{\delta}{ }_{j} G^{\epsilon}{ }_{k}+C_{\alpha \delta} G^{\delta}{ }_{j k}+C_{\alpha \beta \gamma \delta} G^{\delta}{ }_{j} G^{h l}\left[G^{\beta}{ }_{h k} G^{\gamma}{ }_{l}+G^{\beta}{ }_{h} G^{\gamma}{ }_{l k}\right]
$$

after use of (8.15) and (11.8). From these equations and (12.12) we find that

$$
\begin{aligned}
C_{\alpha(j: k)-}= & -C_{\alpha \beta j k: e} G^{\beta \epsilon}+(1 /(m-3))\left[C^{\zeta_{\beta j k: 5}} G^{\beta}{ }_{\alpha}\right. \\
& \left.+G_{\alpha k-}-C_{\beta \gamma j-: 5} G^{\beta \gamma}+(2-n) C^{\zeta}{ }_{\alpha j k: 5}\right] \\
& +C_{\alpha \beta \gamma \delta} G^{\delta}{ }_{j-}-G^{h l}\left[G^{\beta}{ }_{h k-}-G^{\gamma}{ }_{l}+G^{\beta}{ }_{h} G^{\gamma}{ }_{l k-}\right]
\end{aligned}
$$

where, as in (12.18),

$$
\begin{aligned}
& C_{\alpha \beta j k: \epsilon}=C_{\alpha \beta \gamma \delta: e} G_{j}^{\gamma} G^{\delta}{ }_{k} \text {, } \\
& C_{\beta j k: \zeta}=C_{\beta \gamma \delta: \zeta} G^{\gamma}{ }_{j} G^{\delta}{ }_{k} \text {, } \\
& C_{\beta \gamma k: \zeta}=C \zeta_{\beta \gamma \delta: \zeta} G^{\delta}{ }_{k} \text {. }
\end{aligned}
$$

Now, according to (11.11),

$$
C_{i(j: k)^{-}}=\left[C_{\alpha j^{-}} G^{\alpha_{i}}\right]_{: k^{-}}
$$

so that

$$
C_{i(j: k)^{-}}=C_{\alpha(j: k)^{-}}+C_{\alpha(j} G^{\alpha}{ }_{k)-i} .
$$

The results of these last two paragraphs are used in the sequel.

If $(12.1)$ is replaced by

$$
r \geqq 4
$$

the Bianchi identities for the surface Riemann tensor $\kappa^{h}{ }_{i j k}$ may be derived in the same manner as (12.2). They are

$$
\kappa_{i(j k, l)^{+}}=0
$$

and are valid at all points of $V_{n}$. In a similar way, equations like (12.4) to (12.7) hold for the surface objects which are analogous to the corresponding space objects of these equations if $n>2$. We also obtain the following analogues of equations (12.8) and (12.9) respectively:

$$
\begin{aligned}
W^{h}{ }_{i j k: h} & =W^{h_{i j k, h}}+(n-3) W^{h_{i j k} \phi_{, h},} \\
W^{h_{i j k: h}} & =(n-3)\left[\frac{1}{(n-2)} \kappa_{i j k}+W^{h_{i j k} \phi_{, h}}\right],
\end{aligned}
$$

where $\kappa_{i j k}$ is defined by

$$
\kappa_{i j k}=\kappa_{i(j, k)}-\frac{1}{2(n-1)} \kappa_{,\left(i g_{k}\right)-i} .
$$

The method followed in the derivation of (12.12) is valid if $n>3$. In this case, we find the Bianchi identities for the surface Weyl tensor $W^{h}{ }_{i i k}$ : 


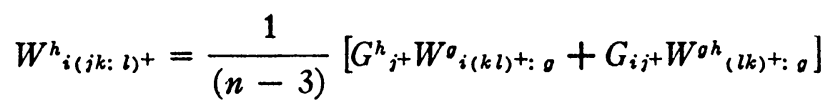

where $W^{o{ }^{h}}{ }_{j l}=W^{o}{ }_{i j l} G^{h i}$.

13. The deviation tensor. In Riemannian geometry, the fundamental forms of a subspace $V_{n}$ are defined in connection with the Frenet equations which describe the variation of the tangent and normal vector spaces of $V_{n}$. In later sections, components of conformal tensors which are analogous to the coefficients of these (metric) fundamental forms are discovered. However, the tensors thus defined are not sufficient for a complete conformal characterization of $V_{n}$ and this fact gives rise to several decisive differences between Riemannian and conformal differential geometry. It is also necessary to investigate the variation of the relative conformal curvature $\Lambda$ and of the mean curvature normal $\mu^{\alpha}$. The first of these two problems is considered in the present section and its solution depends upon the discovery of a new conformal tensor which has no analogue in the metric theory of $V_{n}$. Naturally there are important qualitative differences between the geometric properties of this tensor, defined in connection with the variation of $\Lambda$, and the geometric properties of the other tensors, defined in connection with the Frenet equations.

Suppose (12.23) is satisfied. Then the second partial derivatives of $\Lambda$ (or of $\phi$ ) exist and are continuous. At points of $V_{n}$, covariant differentiation of (8.7) leads to the equations

$$
\begin{aligned}
\sigma_{, i} & =\phi_{, i}-\bar{\phi}_{; i}, \\
\sigma_{, i j} & =\phi_{, i j}-\bar{\phi}_{; i j}-\bar{\phi}_{; i} \sigma_{, i}-\bar{\phi}_{; j} \sigma_{, i}+g_{i j} g^{h k} \sigma_{, i} \bar{\phi}_{; h}
\end{aligned}
$$

since, from (6.3),

$$
\bar{\phi}_{, i j}=\bar{\phi}_{i i j}+\bar{\phi}_{; i} \sigma_{, j}+\bar{\phi}_{; j} \sigma_{, i}-g_{i j} g^{h k} \sigma_{, k} \bar{\phi}_{; h} .
$$

We make the definitions

$$
\begin{aligned}
& \sigma_{i j}=\sigma_{, i j}-\sigma_{, i} \sigma_{, j}, \\
& \phi_{i j}=\phi_{, i j}-\phi_{, i} \phi_{, j}, \\
& \phi_{i j}=\phi_{; i j}-\bar{\phi}_{; i} \bar{\phi}_{; j} .
\end{aligned}
$$

As a consequence of these equations and (13.1) and (13.2),

$$
\sigma_{i j}=\phi_{i j}-\bar{\phi}_{i j}+g_{i j} g^{h k} \sigma_{, k} \Phi_{; h} .
$$

Now

$$
\begin{aligned}
\sigma_{, i} & =\sigma_{, \alpha} g^{\alpha_{i}}, \\
\sigma_{, i j} & =\sigma_{, \alpha \beta} g^{\alpha}{ }_{i} g^{\beta}{ }_{j}+\sigma_{, \alpha} g^{\alpha}{ }_{i j}
\end{aligned}
$$

where $g^{\alpha_{i j}}$ is defined by (5.3). It follows from (11.4), (13.3) and the last two sets of equations that 


$$
\sigma_{i j}=\sigma_{\alpha \beta} g^{\alpha}{ }_{i} g_{j}{ }_{i}+\sigma_{, \alpha} g^{\alpha}{ }_{i j} .
$$

If account is taken of (4.5), (6.10), (11.3), (13.5) and (13.7), we obtain

$$
\begin{aligned}
& {\left[\frac{\rho}{2(m-1)(m-2)} \cdot g_{i j}-\frac{1}{(m-2)} \cdot \rho_{\alpha \beta} g^{\alpha_{i} g^{\beta}}{ }_{i}-\phi_{i j}\right]} \\
& \quad-\left[\frac{\bar{\rho}}{2(m-1)(m-2)} \cdot \bar{g}_{i j}-\frac{1}{(m-2)} \cdot \bar{\rho}_{\alpha \beta} \bar{g}^{\alpha}{ }_{i \bar{g}}{ }^{\beta}{ }_{i}-\bar{\phi}_{i j}\right] \\
& =\sigma_{, \alpha} G^{\alpha}{ }_{i j}+\left[\sigma_{, \alpha} \mu^{\alpha}-a^{\alpha \beta} \sigma_{, \alpha} \sigma_{, \beta} / 2-g^{\left.h k_{\sigma_{k} k} \bar{\phi}_{; h}\right] g_{i j} .}\right.
\end{aligned}
$$

Since $\mu^{\alpha}$ and $G^{\alpha}{ }_{i j}$ are orthogonal to $\phi_{, \alpha}$, it follows from (4.14), (8.8), (8.9) and (8.10) that the expression which appears in the right member of (13.8) is equal to

$$
\left[\mu_{\alpha} G^{\alpha}{ }_{i j}+\left(\mu^{2}-\Delta_{1} \phi\right) g_{i j} / 2\right]-\left[\bar{\mu}_{\alpha} \bar{G}^{\alpha}{ }_{i j}+\left(\bar{\mu}^{2}-\bar{\Delta}_{1} \bar{\phi}\right) \bar{g}_{i j} / 2\right] .
$$

Therefore (13.8) may be written as

where

$$
E_{i j}=\bar{E}_{i j}
$$

$$
\begin{aligned}
E_{i j}= & \mu_{\alpha} G^{\alpha_{i j}}-\phi_{i j}-\frac{1}{(m-2)} \rho_{\alpha \beta} g^{\alpha_{i} g^{\beta}{ }_{j}} \\
& +\frac{1}{2}\left[\mu^{2}-\Delta_{1} \phi+\rho /(m-1)(m-2)\right] g_{i j}
\end{aligned}
$$

and $\bar{E}_{i j}$ is the tensor formed from the corresponding geometric objects of $\bar{V}_{n}$. We call the conformal tensor $E_{i j}$ the deviation tensor $\left.{ }^{(32}\right)$ of $V_{n}$. It is immediate that $E_{i}=E_{j i}$.

The above proof is valid even if $r \geqq 3$ provided $\phi$ is of class $C^{2}$. For, under these assumptions, $\phi_{; i}$ exists and (13.1) holds. Since $\phi_{, i}$ and $\sigma_{, i}$ are of class $C^{1}$, (13.1) shows that the second partial derivatives of $\phi$ exist. Hence $\phi$ is of class $C^{2}$. From this point the previous proof may be continued unchanged. Now (13.9) shows that the existence of $E_{i j}$ with $r \geqq 3$ implies the existence of $\phi_{, i j}$ so that, in this case, $\phi$ is of class $C^{2}$. Hence we may conclude that if $E_{i j}$ exists in a $V_{n}$ with $r \geqq 3, \bar{E}_{i j}$ exists in any corresponding $\bar{V}_{n}$ obtained from $V_{n}$ by an allowable conformal map.

It is convenient to derive another expression for $E_{i j}$ which involves conformal differentiation instead of the usual (metric) differentiation. To attain this end, we write

$$
\psi=-\phi .
$$

It follows from (8.1), (8.5), (13.4) and (13.10) that

(32) A justification for this name depends upon a pictorial interpretation of $E_{i j}$ which will appear elsewhere. 


$$
\psi_{: i}=-\phi_{, i}
$$

$$
\psi_{i j}=-\phi_{i j}-G^{h k} \psi_{: k} \psi_{: k} G_{i j}
$$

where

$$
\psi_{i j}=\psi_{: i j}-\psi_{:} \psi_{: j} \text {. }
$$

According to (7.3), (13.9), (13.10), (13.11) and (13.12), $E_{i j}$ may be written as

$$
\begin{aligned}
E_{i j}= & \psi_{i j}+\mu_{\alpha} G^{\alpha}{ }_{i j}-\frac{1}{(m-2)} \rho_{\alpha \beta} G^{\alpha}{ }_{i} G^{\beta}{ }_{j} \\
& +\frac{1}{2}\left[e^{2 \psi} \mu^{2}+G^{h k} \psi: h \psi: k+e^{2 \psi} \rho /(m-1)(m-2)\right] G_{i j} .
\end{aligned}
$$

From these equations and (9.7), we obtain

$$
{ }^{a} \psi_{i j}=E_{i j}+\frac{1}{(m-2)}{ }^{0} \rho_{\alpha \beta} G^{\alpha}{ }_{i} G^{\beta}{ }_{j}-\frac{{ }^{0} \rho}{2(m-1)(m-2)} G_{i j}
$$

Digression. The proof of the conformal character of $E_{i j}$ which appears above does not depend upon the definition of $\phi$ given by equations (6.12) and (7.1). The only property of $\phi$ which is actually used is that stated in (8.7). Hence if $\chi$ is any other scalar which transforms so that $\sigma=\chi-\bar{\chi}$, a conformal tensor similar to $E_{i j}$ may be constructed by replacing $\phi$ by $\chi$ in (13.9). However, this tensor is closely related to $E_{i j}$ as we now show.

It follows from the transformation laws for $\phi$ and $\chi$ that

$$
\chi=\phi+T
$$

where $T$ is a conformal scalar. Conversely, if $T$ is any conformal scalar, $\chi$ may be defined by (13.16). We denote the conformal tensor obtained by replacing $\phi$ by $\chi$ in (13.9) by $E_{i j}(T)$. An easy calculation based upon (13.16) and the definitions of $E_{i j}(T)$ and $E_{i j}$ leads to the equations

$$
\begin{aligned}
E_{i j}(T)= & E_{i j}-T_{, i j}+T_{, i} T_{, j}+T_{, i} \phi_{, j}+T_{, j} \phi_{, i} \\
& -g^{h k} T_{, k}\left[T_{, k}+2 \phi_{, k}\right] g_{i j} / 2 .
\end{aligned}
$$

According to (8.1) and (8.5) (or by making replacements), these equations may be written as

$$
E_{i j}(T)=E_{i j}-T_{: i j}+T_{: i} T_{: j}-G^{h k} T_{: h} T_{: k} G_{i j} / 2 .
$$

Hence $E_{i j}(T)$ is the sum of $E_{i j}$ and a tensor obtained by the classical method of covariant differentiation. We note that $E_{i j}(0)=E_{i j}$.

14. The conformal Riemann tensor of $V_{n}$. A conformal geometric object which plays the role of the Riemann curvature tensor relative to the surface measure tensor $G_{i j}$ may be constructed if the $\Gamma^{i}{ }_{j k}$ are of class $C^{1}$. This is true if (12.23) holds. It is defined at all points of $V_{n}$ by the equations 


$$
K^{i}{ }_{j k l}=\frac{\partial}{\partial x^{k}} \Gamma^{i}{ }_{j l}-\frac{\partial}{\partial x^{l}} \Gamma^{i}{ }_{j k}+\Gamma^{h}{ }_{j l} \Gamma^{i}{ }_{h k}-\Gamma^{h}{ }_{j k} \Gamma^{i}{ }_{h l}
$$

and is called the conformal Riemann tensor of $V_{n}$ or the surface conformal Riemann tensor. Since the $\Gamma^{i}{ }_{j k}$ are Christoffel symbols, it follows easily that $K^{i}{ }_{j k l}$ is a tensor with respect to parameter transformations. We also define the conformal Ricci tensor of $V_{n}$ (surface conformal Ricci tensor) by the equations

$$
K_{j k}=K^{i}{ }_{j k i}, \quad K^{i}{ }_{k}=G^{i j} K_{j k}
$$

and the conformal curvature of $V_{n}$ (surface conformal curvature) by the equation

$$
K=K_{i j} G^{i j} \text {. }
$$

Of course, $K_{j k}$ is a symmetric tensor and $K^{i}{ }_{j}$ may be defined by the equations $K^{i}{ }_{j}=K^{i}{ }_{k l j} G^{k l}$. If we write $K_{i j k l}=G_{h i} K^{h}{ }_{j k l}$, it is a consequence of (14.1) that $K_{i j k l}$ satisfies the identities

$$
K_{i j k l}=-K_{j i k l}=-K_{i j l k}=K_{k l i j}, \quad K_{i(j k l)+}=0 .
$$

We also note that equations like those below (11.2) may be derived which are based upon (5.14), (8.5), (13.10) and (14.1). These equations are

$$
\kappa_{i j}=K_{i j}+(n-2) \psi_{i j}+G_{i j} G^{h k}\left[\psi_{: h k}+(n-2) \psi_{: k} \psi_{: k}\right]
$$

where $\psi_{i j}$ is defined by (13.13). From these equations and (7.10), (7.11) and (13.14), we obtain

$$
\kappa_{i j}=K_{i j}+(n-2)\left[E_{i j}-\mu_{\alpha} G^{\alpha}{ }_{i j}+\frac{1}{(m-2)} \rho_{\alpha \beta} G^{\alpha}{ }_{i} G^{\beta}{ }_{j}\right]
$$

$$
+G_{i j}\left[E+\frac{1}{(m-2)} \rho_{\alpha \beta} G^{\alpha \beta}-(n-1) e^{2 \psi}\left(\mu^{2}+\frac{\rho}{(m-1)(m-2)}\right)\right]
$$

where we have written

$$
E=E_{i j} G^{i j}
$$

It follows from (14.5) that

$$
\begin{aligned}
{ }^{0} \kappa_{i j}= & K_{i j}+(n-2)\left[E_{i j}+\frac{1}{(m-2)}{ }^{0} \rho_{\alpha \beta} G^{\alpha}{ }_{i} G^{\beta}{ }_{j}\right] \\
& +G_{i j}\left[E+\frac{1}{(m-2)}{ }^{0} \rho_{\alpha \beta} G^{\alpha \beta}-\frac{(n-1)^{0} \rho}{(m-1)(m-2)}\right] .
\end{aligned}
$$

If we assume that

$$
r \geqq 5
$$


the first derivatives of the $K^{h}{ }_{i j k}$ exist. In this case, by means of conformal geodesic parameters, the Bianchi identities for the surface conformal Riemann tensor may be derived following the proof in the metric case. These identities are

$$
K^{h}{ }_{i(j k: l)^{+}}=0 .
$$

From these equations, other results such as the equations

$$
G^{i j} K_{i l: j}=K_{: l} / 2
$$

which are analogous to (12.3) may be obtained.

Since, according to (7.3), the correspondence between the tensors $G_{i j}$ and $g_{i j}$ is a conformal one, the Weyl tensors formed with respect to $G_{i j}$ and $g_{i j}$ are each equal to $W^{h}{ }_{i j k}$. Consequently, if $n>2$, the equations analogous to (12.4) and (12.7) are

$$
W_{i(j k: l)^{+}}=\frac{1}{(n-2)}\left[G^{h}{ }_{j^{+}} K_{i(k l)^{+}}+G_{i j^{+}} K^{h}(l k)^{+}\right]
$$

and

$$
W_{i j k: h}^{h}=\frac{(n-3)}{(n-2)} K_{i j k}
$$

where

$$
K_{i j k}=K_{i(j: k)^{-}}+\frac{1}{2(n-1)} \cdot K_{:(j} G_{k)^{-i}}, \quad K^{h}{ }_{j k}=G^{h i} K_{i j k} .
$$

15. Variation of the mean curvature normal. We assume that (5.13) is true. Then generalized covariant differentiation of (6.10) leads to the equations

$$
G^{\alpha}{ }_{i h, l}=g^{\alpha}{ }_{h, i l}-\mu^{\alpha},{ }_{,} g_{i h} .
$$

Now the Ricci identities for this type of differentiation show that

$$
g^{\alpha}{ }_{h,(i l)^{-}}=g^{\alpha}{ }_{j k^{j}{ }^{j}{ }_{h i l}}-g^{\beta}{ }_{h} g^{\gamma}{ }_{i}{ }^{\delta}{ }_{l \rho^{\alpha}}{ }_{\beta \gamma \delta} .
$$

If the value of $g^{\alpha, i l}$ from these equations is substituted in (15.1) and the result is multiplied by $g^{h l}$ and summed, we obtain

$$
(n-1) \mu^{\alpha}{ }_{, i}=g^{h l} G^{\alpha}{ }_{i h, l}+g^{\alpha}{ }_{h K^{h}{ }_{i}}+g^{\gamma}{ }_{i} g^{\beta \delta} \rho^{\alpha}{ }_{\beta \gamma \delta}
$$

after simplification by means of (4.10), (5.6) and (5.14).

Now, according to the definitions which appear in (5.2) and (8.1) as well as (7.11), (8.5), (8.10), (8.11) and (13.10), it follows that

$$
\begin{aligned}
g^{h l} G^{\alpha}{ }_{i h: l} & =\left[G^{\alpha}{ }_{i h, l}-(n-1) G^{\alpha}{ }_{i h} \psi_{: l}+\mu_{\beta} G^{\beta}{ }_{i n} g^{\alpha}{ }_{l}\right] g^{h l}, \\
\mu^{\alpha}: i & =\mu^{\alpha}{ }_{, i}-\mu^{\alpha} \psi_{: i}+\mu^{2} g^{\alpha}{ }_{i} .
\end{aligned}
$$


To obtain these equations, use has also been made of the fact that both $G^{\alpha_{i j}}$ and $\mu^{\alpha}$ are orthogonal to $g^{\alpha}{ }_{i}$. As a consequence of these equations, (15.2) is equivalent to

$$
\begin{aligned}
(n-1) \mu^{\alpha}: i= & e^{-2 \psi} G^{h l}\left[G^{\alpha}{ }_{i h: l}+(n-1) G^{\alpha}{ }_{i h} \psi: l-\mu_{\beta} G^{\beta}{ }_{i h} G^{\alpha}{ }_{l}\right] \\
& +(n-1) \mu^{2} G^{\alpha}{ }_{i}-(n-1) \mu^{\alpha} \psi_{: i}+G^{\alpha}{ }_{h} \kappa^{{ }^{h}}{ }_{i} \\
& +e^{-2 \psi} G^{\gamma}{ }_{i} G^{\beta \delta} \rho^{\alpha}{ }_{\beta \gamma \delta} .
\end{aligned}
$$

If assumption (5.13) is replaced by (12.23), we may substitute the value for $\kappa_{i j}$ given by (14.5) in (15.3). If this is done and we then make use of (11.5) and (11.6), we obtain

$$
\begin{aligned}
& (n-1) \mu^{\alpha}: i=e^{-2 \psi}\left[G^{\alpha}{ }_{i h: l} G^{h l}+(n-1) G^{\alpha}{ }_{i h} G^{h l} \psi_{: l}-(n-1) e^{2 \psi_{\mu} \alpha} \psi_{: i}\right. \\
& -(n-1) \mu_{\gamma} G^{\gamma_{i h}} G^{\alpha}{ }_{l} G^{h l}+G^{\alpha}{ }_{h} K^{h_{i}}+(n-2) G^{\alpha}{ }_{h} E^{h_{i}} \\
& \left.+G^{\alpha}{ }_{i} E-C^{\alpha}{ }_{\beta} G^{\beta}{ }_{i}-((n-1) /(m-2)) H^{\alpha \beta}{ }_{\rho_{\beta} \gamma} G^{\gamma_{i}}\right] \text {. }
\end{aligned}
$$

These equations, or (15.2), or (15.3) describe the variation of $\mu^{\alpha}$. From (9.8) and (15.3), we find that

$$
(n-1)^{0} \mu^{\alpha}: i=G^{\alpha}{ }_{i h: l} G^{h l}+G^{\alpha}{ }_{h}^{0} \kappa^{h}{ }_{i}+G^{\gamma}{ }_{i} G^{\beta \delta}{ }^{0} \rho_{\beta \gamma \delta}^{\alpha}
$$

and from (9.8) and (15.4), we obtain

$$
\begin{aligned}
(n-1)^{0} \mu^{\alpha}: i= & G^{\alpha}{ }_{i h: l} G^{h l}+G^{\alpha}{ }_{h} K^{h}{ }_{i}+(n-2) G^{\alpha}{ }_{h} E^{h}{ }_{i}+G^{\alpha}{ }_{i} E \\
& -C^{\alpha} G_{\beta} G_{i}-((n-1) /(m-2)) \cdot H^{\alpha \beta}{ }^{0}{ }_{\rho_{\beta}} G^{\gamma}{ }_{i} .
\end{aligned}
$$

16. The conformal Riemann tensor of $V_{m}$. Since the space measure tensor $A_{\alpha \beta}$ is defined only on $V_{n}$, it is not possible to construct a Riemann curvature tensor relative to $A_{\alpha \beta}$ throughout $V_{m}$. A complete analogue does not even exist at points of $V_{n}$ since $\partial \Gamma_{\beta \gamma}^{\alpha} / \partial y^{\delta}$ is not defined. However, if (12.23) holds, the quantities $R^{\alpha}{ }_{\beta j k}$ are defined on $V_{n}$ by the equations

(16.1) $\quad R_{\beta j k}^{\alpha_{\beta j}}=G^{\delta_{k}} \frac{\partial}{\partial x^{j}} \Gamma_{\beta \delta}^{\alpha_{\beta \delta}}-G^{\gamma}{ }_{j} \frac{\partial}{\partial x^{k}} \Gamma_{\beta \gamma}^{\alpha_{\beta \gamma}}+\left[\Gamma_{\beta \delta}^{\epsilon_{\beta \delta}} \Gamma^{\alpha}{ }_{\epsilon \gamma}-\Gamma_{\beta \gamma}^{\epsilon_{\beta \gamma}} \Gamma^{\alpha}{ }_{\epsilon \delta}\right] G_{j}^{\gamma} G_{k}^{\delta_{k}}$

Formally, $R^{\alpha}{ }_{\beta j k}$ is equal to $R^{\alpha}{ }_{\beta \gamma \delta} G^{\gamma} G_{j}{ }^{\delta}$ where $R^{\alpha}{ }_{\beta \gamma \delta}$ is the (non-existent) formal Riemann tensor constructed from the symbols $\Gamma^{\alpha}{ }_{\beta \gamma}$. This leads to the conjecture that $R^{\alpha}{ }_{\beta j k}$ is a tensor with respect to coordinate-parameter transformations. The correctness of this guess may be verified by a direct calculation or by other methods. We call $R^{\alpha}{ }_{\beta j k}$ the conformal Riemann tensor of $V_{m}$ or the space conformal Riemann tensor. We also define the conformal Ricci tensor of $V_{m}$ (space conformal Ricci tensor) by the equations

$$
R^{\alpha}{ }_{k}=R^{\alpha}{ }_{\beta j k} G^{\beta j}
$$

and the conformal curvature of $V_{m}$ (space conformal curvature) by the equation

$$
R=R^{\alpha}{ }_{k} G_{\alpha}^{k} \text {. }
$$


If we write

$$
\begin{array}{rlrl}
R_{\alpha \beta j k} & =A_{\alpha \gamma} R^{\gamma_{\beta j k}}, & R_{\alpha k}=A_{\alpha \beta} R^{\beta}{ }_{k}, \\
R^{\alpha_{i j k}}=R^{\alpha_{\beta j k}} G^{\beta}, & R_{j k}=R_{\alpha k} G^{\alpha}{ }_{j}, \\
R_{h i j k}=R_{\alpha \beta j k} G^{\alpha} G^{\beta}{ }_{i} &
\end{array}
$$

it follows from (16.1) and (16.4) that

$$
\begin{aligned}
R_{\alpha \beta j k} & =-R_{\beta \alpha j k}=-R_{\alpha \beta k j}, \\
R^{\alpha}{ }_{(i j k)^{+}} & =0, \\
R_{h i j k} & =-R_{i h j k}=-R_{h i k j}=R_{j k h i}, \\
R_{h(i j k)^{+}} & =0 \\
R_{i j} & =R_{h i j k} G^{h k}=R_{i h k} G^{h k}, \quad R_{i j}=R_{j i} .
\end{aligned}
$$

These equations may be derived by direct calculation or by making use of the identities satisfied by the formal tensor $R^{\alpha}{ }_{\beta \gamma \delta}$.

Assumption (14.8) guarantees the existence of $R_{\beta j k: i}^{\alpha}$. If we choose conformal geodesic coordinates and parameters at a point $P$ of $V_{n}$, differentiation of (16.1) at $P$ yields

$$
\begin{aligned}
R_{\beta j k: i}^{\alpha}= & G^{\delta}{ }_{k} \frac{\partial^{2}}{\partial x^{i} \partial x^{j}} \Gamma_{\beta \delta}^{\alpha}-G^{\gamma}{ }_{j} \frac{\partial^{2}}{\partial x^{i} \partial x^{k}} \Gamma_{\beta \gamma}^{\alpha} \\
& +G^{\delta}{ }_{k i} \frac{\partial}{\partial x^{j}} \Gamma_{\beta \gamma}^{\alpha}-G^{\gamma}{ }_{j i} \frac{\partial}{\partial x^{k}} \Gamma_{\beta \gamma}^{\alpha} .
\end{aligned}
$$

From this result and similar equations, we obtain $R_{\beta(j k: i)+}^{\alpha}=0$ at $P$. It is clear that these equations hold in all coordinate-parameter systems and at all points of $V_{n}$. They are the Bianchi identities for the conformal Riemann tensor of $V_{m}$.

It is possible to express $R^{\alpha}{ }_{\beta j k}$ in terms of other tensors which have already been defined. To simplify the calculations, we choose conformal geodesic coordinates at a point $P$ of $V_{n}$. Since (16.1) are conformal equations, the replacement principle may also be employed. It follows from (9.9) that the coordinates are geodesic at $\mathscr{P}$, that is,

$$
\left\{\begin{array}{c}
\alpha \\
\beta \gamma
\end{array}\right\}=0
$$

at $\boldsymbol{P}$. However, the results obtained are true in all coordinate-parameter systems and at all points of $V_{n}$. Now (16.1) becomes

$$
R_{\beta j k}^{\alpha}=G^{\delta}{ }_{k}^{0}\left(\frac{\partial}{\partial x^{j}} \Gamma_{\beta \delta}^{\alpha}\right)-G^{\gamma}{ }_{i}^{0}\left(\frac{\partial}{\partial x^{k}} \Gamma_{\beta \gamma}^{\alpha}\right) \text {. }
$$

According to (8.11) and (9.7) this may be written as 


$$
\begin{aligned}
R^{\alpha}{ }_{\beta j k}= & G^{\gamma}{ }_{j} G^{\delta}{ }_{k}\left[\frac{\partial}{\partial y^{\gamma}}\left\{\begin{array}{c}
\alpha \\
\beta \delta
\end{array}\right\}-\frac{\partial}{\partial y^{\delta}}\left\{\begin{array}{c}
\alpha \\
\beta \gamma
\end{array}\right\}\right]+G^{\alpha}{ }_{k}{ }^{0} \eta_{\beta: k} \\
& -G^{\alpha}{ }_{i}{ }^{0} \eta_{\beta: k}-G_{\beta k} A^{\alpha \epsilon}{ }^{0} \eta_{\epsilon: j}+G_{\beta j} A^{\alpha \epsilon}{ }^{0} \eta_{\epsilon: k} .
\end{aligned}
$$

To obtain these equations, we have made use of the fact that ${ }^{0} \eta_{\gamma: k} G{ }_{j}$ is symmetric in the indices $j, k$. To prove this we note that conformal differentiation of $\eta_{\gamma} G r_{j}=-\psi_{: j}$ gives, using (8.15),

$$
\eta_{\gamma: k} G^{\gamma}{ }_{j}+\eta_{\gamma} G^{\gamma}{ }_{j k}=-\psi_{: j k} .
$$

Since $\psi_{: j k}$ and $G^{\gamma}{ }_{j k}$ are symmetric in the lower indices this is also true for $\eta_{\gamma: k} G^{\boldsymbol{\gamma}_{j}}$ and hence for ${ }^{0} \eta_{\gamma: k} G^{\boldsymbol{\gamma}_{j}}$.

Now from (8.8), (8.10), (13.10), (9.7) and (9.8), ${ }^{0} \eta_{\beta: j}={ }^{0} \mu_{\beta: j}-{ }^{0}\left(\psi_{h j}\right) G^{h}{ }_{\beta}$. As a consequence of (13.15), (14.7) and (15.5), the above equations become

$$
\begin{aligned}
(n-1)^{0} \eta_{\beta: j}= & A_{\epsilon \beta} G^{\epsilon}{ }_{j h: i} G^{h i}+G^{\gamma} G_{j}^{\epsilon \delta}{ }^{0} \rho_{\beta \epsilon \gamma \delta} \\
& +G^{h}{ }_{\beta}\left[K_{h j}-E_{h j}+E G_{h j}-(1 /(m-2))^{0} \rho_{\epsilon \gamma} G^{\epsilon}{ }_{h} G^{\gamma}{ }_{j}\right. \\
& +(1 /(m-2)){ }^{0} \rho_{\epsilon \gamma} G^{\epsilon \gamma} G_{h j} \\
& \left.-\left((n-1)^{0} \rho / 2(m-1)(m-2)\right) G_{h j}\right] .
\end{aligned}
$$

Since the coordinates are geodesic, (5.14) shows that

$$
{ }^{0} \rho_{\beta \gamma \delta}^{\alpha}=\frac{\partial}{\partial y^{\gamma}}\left\{\begin{array}{c}
\alpha \\
\beta \delta
\end{array}\right\}-\frac{\partial}{\partial y^{\delta}}\left\{\begin{array}{c}
\alpha \\
\beta \gamma
\end{array}\right\} .
$$

If this result and (16.7) are substituted in (16.6), after some simplification we obtain

$$
\begin{aligned}
& R^{\alpha}{ }_{\beta j k}=\left\{C^{\alpha \beta \delta}+\frac{1}{(n-1)}\left[A^{\alpha}{ }_{(\gamma} C_{\delta)-\beta}+C^{\alpha}{ }_{(\gamma} A_{\delta)-\beta}\right]\right\} G^{\gamma}{ }_{j} G^{\delta}{ }_{k} \\
& +\frac{1}{(n-1)}\left\{E^{l_{k}-}\left[G^{\alpha}{ }_{j}-G_{\beta l}-G^{\alpha}{ }_{l} G_{\beta \zeta}-\right]+2 E G^{\alpha}{ }_{k}-G_{\beta j}-\right\} \\
& +\frac{1}{(n-1)} \cdot K^{l}{ }_{j}-\left[G^{\alpha}{ }_{k}-G_{\beta l}-G^{\alpha}{ }_{l} G_{\beta k^{-}}\right] \\
& +\frac{G^{o l}}{(n-1)}\left[A_{\beta \gamma} G^{\alpha}{ }_{(k} G_{j)^{-} g: l}^{\gamma}+G_{\beta(i ;} G^{\alpha}{ }_{k)^{-} \varrho: l}\right]
\end{aligned}
$$

where we have written $E^{i}{ }_{j}=G^{h i} E_{h j}$ and have also used the definitions stated in (11.5) and (11.8).

It is shown in $\$ 22$ that (16.8) may be simplified by means of the conformal analogues of the Gauss equations $\left({ }^{33}\right)$ and written as

(33) The "conformal Frenet equations" (20.7) and the "derived condition" (21.9) with $w=1$ are also used in this derivation. 


$$
\begin{aligned}
R_{\beta j k}^{\alpha}=D^{\alpha}{ }_{\beta j k}+E^{l} k^{-} & {\left[G^{\alpha}{ }_{j}-G_{\beta l}-G^{\alpha} G_{\beta j}\right] } \\
& \left.\cdot \frac{G^{o l}}{(n-1)}\left[H^{\alpha}{ }_{\gamma} G_{\beta(j} G^{\gamma}{ }_{k)^{-0: l}}+H_{\gamma_{\beta}} G^{\alpha}{ }_{(k} G^{\gamma}{ }^{\gamma}\right)^{-0: l}\right]
\end{aligned}
$$

where $D^{\alpha}{ }_{\beta j k}$ is defined by (11.18). This expression does not involve $K_{i j}$. From these equations and (16.2), (16.3) and (16.4) as well as (4.12), (11.8), (11.10) and (11.11), we find that

$$
\begin{aligned}
& R^{\alpha_{i j k}}=D^{\alpha_{i j k}}+G^{\alpha}{ }_{(j} E_{k)^{-i}}+G^{\alpha}{ }_{l} E^{l}{ }_{(j} G_{k)^{-i}} \\
& +(1 /(n-1)) G^{o l} H^{\alpha}{ }_{\gamma} G_{i(j} G^{\gamma}{ }_{k)^{-},:} l \text {, } \\
& R_{h i j k}=C_{h i j k}+G_{h(j} E_{k)-i}+G_{i(k} E_{j)-h} \text {, } \\
& R^{\alpha}{ }_{k}=C_{\beta k} G^{\beta \alpha}-(n-2) G^{\alpha}{ }_{l} E^{l}{ }_{k}-E G^{\alpha}{ }_{k}+G^{0}{ }^{l} H^{\alpha}{ }_{\gamma} G^{\gamma}{ }_{k 0:}{ }_{l} \text {, } \\
& R_{j k}=C_{j k}-(n-2) E_{j k}-E G_{j k} \text {, } \\
& R=C-2(n-1) E .
\end{aligned}
$$

If $n>2$, we may apply the $\uparrow$ operation with respect to $G_{i j}$ to (16.11). From (11.27) and (11.28),

$$
\uparrow C_{h i j k}=\uparrow R_{h i j k}
$$

where, in accordance with (11.25),

$$
\uparrow C_{h i j k}=C_{h i j k}+\frac{1}{(n-2)}\left[G_{h(i} C_{k)^{-i}}+G_{i(k} C_{i)^{-h}}\right]
$$

$$
\begin{gathered}
+\frac{C}{(n-1)(n-2)} G_{h(k} G_{j)^{-i}}, \\
\uparrow R_{h i j k}=R_{h i j k}+\frac{1}{(n-2)}\left[G_{h\left(j R_{k}\right)^{-i}}+G_{i(k} R_{j)^{-} h}\right] \\
+\frac{R}{(n-1)(n-2)} G_{h(k} G_{j)^{-i}} .
\end{gathered}
$$

Each member of these equations is formed in a manner exactly analogous to that used in the construction of the Weyl tensor $W_{h i j k}$.

Finally, we note that $R_{\alpha \beta j k}$ is defined if $\Gamma_{\beta \gamma}^{\alpha}$ is of class $C^{1}$ in the $x^{i}$. Also, if the $\Gamma^{i}{ }_{j k}$ exist and $r \geqq 3$,

$$
\left\{\begin{array}{c}
\alpha \\
\beta \gamma
\end{array}\right\}, \quad\left\{\begin{array}{c}
i \\
j k
\end{array}\right\}
$$

are of class $C^{1}$. It is readily found from (8.5) and (8.11) that $\phi_{, i}$ and $\eta_{\alpha}$ exist and are of class $C^{1}$. Hence $\Gamma^{i}{ }_{j k}$ are also of class $C^{1}$. Since $\Gamma^{i}{ }_{j k}$ and $\Gamma^{\alpha}{ }_{\beta \gamma}$ exist, it follows from (8.4) that they are of class $C^{1}$. It is then found that all the work of this section is valid. This shows that if $\Gamma_{\beta \gamma}^{\alpha}$ is of class $C^{1}$ and $r \geqq 3$ then $R_{\alpha \beta j k}, \bar{R}_{\alpha \beta j k}$ exist and (16.9) are valid. 
17. The Ricci identities. The equality of the differential operators $\partial^{2} / \partial x^{i} \partial x^{i}$ and $\partial^{2} / \partial x^{i} \partial x^{i}$ may be conveniently expressed in terms of covariant differentiation based upon the coefficients of connection $\left({ }^{34}\right) \Gamma^{\alpha}{ }_{\beta \gamma}, \Gamma^{i}{ }_{j k}$. By direct calculation $\left({ }^{35}\right)$ using the definitions which appear in (8.1), (14.1) and (16.1), we obtain

$$
\begin{aligned}
\pi^{\alpha}:(j k)^{-} & =-\pi^{\beta} R^{\alpha}{ }_{\beta j k}, \\
\pi^{\alpha}{ }_{i:(j k)^{-}} & =\pi^{\alpha}{ }_{h} K^{h}{ }_{i j k}-\pi^{\beta}{ }_{i} R^{\alpha}{ }_{\beta j k} .
\end{aligned}
$$

Similar equations for more complicated tensors are also valid. These tensor equations are the Ricci identities. They are used frequently in the sequel to express the integrability conditions of the various partial differential equations which occur in our work.

\section{The CONFORMAL VeCTOR SPACES OF $V_{n}$}

The purpose of this chapter is to define the various osculating and normal conformal vector spaces of $V_{n}$. Associated with these vector spaces are certain important tensors analogous to the "mass tensors" of the metric theory. As in the metric case, a system of Frenet equations is obtained which describes the variation of the vector spaces. The entire development is accomplished without any recourse to beins which span the various vector spaces. A similar theory, which utilizes vector beins, may be developed by our methods. The results of this latter theory are undoubtedly useful for the solution of various special problems which depend upon particular vector beins. The motivation behind our present work is to avoid the introduction of any elements (such as bein indices) which are extraneous to the purely geometric questions which are considered here. The comparable development of the metric theory (at least for spaces of constant curvature) has been given by Mayer [10].

18. The conformal osculating and normal vector spaces of $V_{n}$. At an arbitrary point $\mathcal{P}$ of $V_{n}$, the space vectors

$$
G^{\alpha}
$$

span an $n$-dimensional vector space in accordance with $\$ 4(\mathrm{~d})$. As previously defined, this space is called the tangent vector space of $V_{n}$. Since (18.1) is a conformal geometric object, we also name the space the first conformal osculating vector space of $V_{n}$ and denote it by the symbol $I_{1}$.

Suppose

$$
x^{\prime i}=x^{\prime i}\left(x^{j}\right), \quad x^{i}=x^{i}\left(x^{\prime j}\right)
$$

(अ) No assumption is made that $\Gamma^{i}{ }_{j k}, \Gamma_{\beta \gamma}^{\alpha}$ have the meaning given them by equations (8.5) and (8.11) respectively. The quantities $\Gamma^{i}{ }_{j k}, \Gamma^{\alpha}{ }_{\beta \gamma}$ may be any surface and space coefficients of connection and $K^{h_{i j k}}, R^{\alpha}{ }_{\beta j k}$ are the tensors defined in terms of $\Gamma^{i}{ }_{j k}, \Gamma^{\alpha}{ }_{\beta \gamma}$ by equations (14.1) and (16.1) respectively.

(35) These calculations may be simplified by choosing coordinates and parameters which are geodesic with respect to $\Gamma^{i}{ }_{i k}, \Gamma_{\beta \gamma}^{\alpha}$. 
is an allowable parameter transformation. The symbols which denote geometric objects in the parameter system $\left\{x^{\prime i}\right\}$ are written with primes to dis: tinguish them from the corresponding symbols in the parameter system $\left\{x^{i}\right\}$. Then

$$
G^{\prime \alpha_{i}}=G^{\alpha}{ }_{j} \partial x^{j} / \partial x^{\prime i}, \quad G^{\alpha_{i}}=G^{\prime \alpha}{ }_{j} \partial x^{\prime j} / \partial x^{i} .
$$

Consequently $I_{1} \equiv I_{1}{ }^{\prime}$ and the tangent vector space does not depend upon the parameterization of $V_{n}$.

The second conformal osculating vector space $I_{12}$ of $V_{n}$ at $P$ is defined as the totality of space vectors at $P$ which are spanned linearly by the space vectors $G^{\alpha}{ }_{i}, G^{\alpha}{ }_{i: j}$. It is immediate that this vector space is unchanged by conformal transformations of $V_{m}$. It is also invariant under parameter changes as can be seen from the transformation law between $G^{\alpha_{i: j}}$ and $G^{\prime \alpha_{i: j}}$. This law as well as the one connecting $G^{\alpha}{ }_{i}$ and $G^{\prime \alpha_{i}}$ which appears above are special cases of the general formula

$$
\begin{aligned}
& G^{\prime \alpha_{i_{1}: i_{2}} \cdots i_{w}}=G^{\alpha}{ }_{j_{1}: j_{2} \cdots j_{w}} \frac{\partial x^{i_{1}}}{\partial x^{\prime i_{1}}} \cdots \frac{\partial x^{j_{v}}}{\partial x^{\prime i_{v}}}+\sum_{v=1}^{w-1} G^{\alpha}{ }_{j_{1}: j_{2}} \cdots j_{v}(\cdots),
\end{aligned}
$$

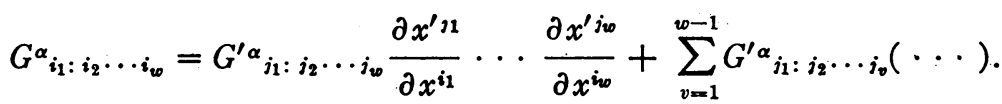

The wth conformal osculating vector space $I_{12} \ldots w$ of $V_{n}$ at $P$ is defined as the totality of space vectors at $\boldsymbol{P}$ which are spanned linearly by the space vectors $G^{\alpha_{i_{1}}}, G^{\alpha_{i_{1}: i_{2}}}, \cdots, G^{\alpha_{i_{1}: i_{2}}} \ldots i_{i_{w}}$. The invariance of $I_{12} \ldots w$ with respect to parameter changes is a consequence of (18.3). Of course, $I_{12} \ldots w$ is invariant with respect to coordinate transformations. Its conformal character follows from its definition and the properties of conformal differentiation. The osculating spaces are so defined that $I_{12} \ldots w$ contains $I_{12} \ldots w-1$.

Since orthogonality is a conformal geometric property, the set of space vectors belonging to $I_{12} \ldots w$ which are normal to $I_{12} \ldots w-1$ form a conformal linear vector space $I_{w}$. We call $I_{w}$ the $(w-1)$ th conformal normal vector space of $V_{n}$ at $P$. According to this definition, $I_{w}$ is the vector space which is spanned by the projection of $G_{i_{1}: i_{2}} \ldots i_{i_{w}}$ into the vector space which is orthogonal to $I_{12} \ldots w-1$. We denote this projection $\left.{ }^{(36}\right)$ of $G^{\alpha_{i_{1} i_{2}} \ldots i_{w}}$ by $G^{\alpha_{i_{1} i_{2}} \ldots i_{w}}{ }$. Of course $G^{\alpha}{ }_{i_{1} i_{2}} \ldots i_{i_{w}}$ is a conformal geometric object. It transforms as a contravariant vector with respect to coordinate transformations. It follows from (18.3) that

$$
\begin{aligned}
& G^{\prime \alpha_{i_{1} i_{2}} \cdots i_{i_{v}}}=G^{\alpha}{ }_{j_{1} j_{2} \cdots j_{v}} \frac{\partial x^{i_{1}}}{\partial x^{\prime i_{1}}} \cdots \frac{\partial x^{j_{v}}}{\partial x^{\prime i_{0}}}, \\
& G_{i_{1} i_{2}}^{\alpha_{1} \ldots i_{w}}=G^{\prime \alpha}{ }_{j_{1} j_{2}} \ldots j_{w} \frac{\partial x^{\prime j_{1}}}{\partial x^{i_{1}}} \cdots \frac{\partial x^{\prime j_{\omega}}}{\partial x^{i_{\omega}}} .
\end{aligned}
$$

Consequently $G_{i_{i_{1}}{ }_{2}}^{\alpha_{2} \ldots i_{i_{w}}}$ transforms like a covariant surface tensor of the wth

(2) The projection tensor of $I_{w}$ should be defined relative to $A_{\alpha \beta}$ and is a conformal tensor. 
order under changes of parameter. Using the notation of $\$ 1$, we frequently write $G^{\alpha_{i_{1} i_{2}} \ldots i_{i_{w}}}$ as $G^{\alpha}{ }_{\left(i_{w}\right)}$.

The construction of successive osculating vector spaces continues to lead to new spaces until we reach an osculating space $I_{12} \ldots M$ which has the property

$$
I_{12} \ldots M \neq I_{12} \cdots M-1, \quad I_{12} \cdots M+1=I_{12} \cdots M .
$$

It follows from (18.4) that

$$
I_{12 \cdots M+u}=I_{12 \cdots M}
$$

if $u \geqq 0$. Consequently we call $I_{12} \ldots M$ the last conformal osculating vector space of $V_{n}$. It is clear from these remarks and the various definitions that

$$
\begin{gathered}
I_{w} \neq 0, \quad w \leqq M, \\
I_{M+1} \equiv 0,
\end{gathered}
$$

that is, $I_{M+1}$ is the first empty space. Each succeeding normal vector space also has dimension zero.

We denote the dimension of $I_{w}$, which is identical with the rank of the matrix $\left\|G^{\alpha}{ }_{\left(i_{w}\right)}\right\|$ by $N_{w}$. Then the dimension $N_{12} \ldots$ of $I_{12} \ldots$ is equal to

$$
N_{12 \cdots w}=N_{1}+N_{2}+\cdots+N_{w} \text {. }
$$

Of course, $N_{1}=n$. Equations (18.5) are equivalent to

$$
N_{w}>0, \quad w \leqq M
$$

and (18.6) to $N_{M+1}=0$. The existence of an integer $M$ for which (18.6) holds follows from (18.7), (18.8) and the inequalities

$$
N_{12 \cdots w} \leqq m \text {. }
$$

Of course, (18.9) is true because the osculating vector spaces all lie in $V_{m}$.

It is clear that (18.6) is equivalent to

$$
G^{\alpha}{ }_{\left(i_{M+1}\right)}=0 \text {. }
$$

The space vectors

$$
G_{i_{1}}^{\alpha_{1}}, G^{\alpha}{ }_{\left(i_{2}\right)}, \cdots, G^{\alpha}{ }_{\left(i_{M}\right)}
$$

constitute a basis for the conformal osculating spaces and conformal normal spaces of $V_{n}$. We note that if $r \geqq 3$, each successive conformal basis bein $G^{\alpha}{ }_{\left(i_{w}\right)}$ exists if the preceding basis bein $G^{\alpha}{ }_{\left(i_{w}-1\right)}$ exists and is of $\operatorname{class}\left({ }^{\left({ }^{7}\right)} C^{1}\right.$. A suffi-

(87) In making this statement, we are thinking of an alternative method of development of this section. In this other method, we first define the vector spaces $I_{1}, I_{2}, \cdots, I_{w}$ and afterwards define $I_{12} \ldots$ as the linear vector space spanned by the vectors of $I_{1}, I_{2}, \ldots, I_{w}$. The normal vector space $I_{\infty}$ is defined as the linear vector space which is spanned by the space vectors $G_{t_{0-1}} t_{t_{0}}$ which are normal to $I_{12} \ldots \infty-1$. 
cient but not necessary condition that all the beins of the basis (18.11) exist and be of class $C^{1}$ is that the inequality

$$
r \geqq M+1
$$

be true. If each bein of (18.11) is of class $C^{*}$, we say that (18.11) is a basis of class $C^{s}$. We also remark that if the basis beins $G^{\alpha}{ }_{\left(i_{w}\right)}$ exist and are of class $C^{1}$ in a $V_{n}$ with $r \geqq 3$, then the corresponding basis beins $\bar{G}^{\alpha_{\left(i_{w}\right)}}$ in any $\bar{V}_{n}$ obtained by an allowable conformal transformation of the enveloping $V_{m}$ exist and are equal to the $G^{\alpha}{ }_{\left(i_{w}\right)}$.

Simply as a matter of convenient notation and without further significance, we agree to write $I_{\infty}$ for the vector space which is orthogonal to all the conformal osculating vector spaces of $V_{n}$. We write $G^{\alpha}{ }_{\left(i_{\infty}\right)}$ for any set of space vectors which span $I_{\infty}$. Hence

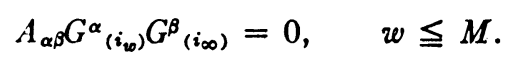

If we write $N_{\infty}$ for the dimension number of $I_{\infty}$, it follows that $N_{1}+N_{2}+\cdots$ $+N_{M}+N_{\infty}=m$.

19. The conformal normal measure tensors. Any space vector $L^{\alpha}$ which lies entirely in the tangent vector space of $V_{n}$ may be written as

$$
L^{\alpha}=G^{\alpha} L^{i} \text {. }
$$

The surface vector $L^{i}$ is the parameter representation of $L^{\alpha}$. Similarly, if $X^{\alpha}$ is any space vector which lies entirely in $I_{w}(w \leqq M)$, it has a representation of the form

$$
X^{\alpha}=G^{\alpha}{ }_{\left(i_{w}\right)} X^{\left(i_{0}\right)} .
$$

It is clear that the quantities $X^{\left(i_{w}\right)}$ which appear in this parameter representation of $X^{\alpha}$ behave like the components of a contravariant surface tensor of the $w$ th order with respect to parameter transformations. The conformal basis beins $G^{\alpha}{ }_{\left(i_{w}\right)}$ enjoy various symmetry properties in the covariant indices $i_{1}, i_{2}, \cdots, i_{w}$ which are derived in $\S 27$. The components of $X^{\left(i_{w}\right)}$ may be assumed to have these same symmetry properties without loss of generality. We denote the number of different components of $X^{\left(i_{w}\right)}$ by $N_{w}^{\prime}$.

In general, if $w>1$ the space vectors $G^{\alpha}{ }_{\left(i_{w}\right)}$ are not linearly independent. Consequently the null vector also has a representation in $I_{w}$ of the form

$$
0=G^{\alpha}{ }_{\left(i_{w}\right)} \theta^{\left(i_{0}\right)}
$$

where not all the components of $\theta^{\left(i_{w 0}\right)}$ vanish. We call $\theta^{\left(i_{w}\right)}$ a null tensor or a null solution. The number of linearly independent solutions of (19.3) is $N_{w}^{\prime}-N_{w}$. In the representation of $X^{\alpha}$ which appears in (19.2), the tensor $X^{\left(i_{w}\right)}$ is determined up to an additive null solution $\theta^{\left(i_{w}\right)}$ of equations (19.3). We call any of these tensors $X^{\left(i_{w}\right)}$ a contravariant (parameter) representation of the vector $X^{\alpha}$. 
An analogous representation for the covariant vector $X_{\alpha}$ which corresponds to $X^{\alpha}$ may be defined by the equations

$$
X_{\left(i_{w}\right)}=X_{\alpha} G^{\alpha}{ }_{\left(i_{w}\right)} \text {. }
$$

The $X_{\left(i_{w}\right)}$ are the components of a covariant surface tensor of the wth order. We call $X_{\left(i_{w}\right)}$ the covariant (parameter) representation of the vector $X_{\alpha}$. In contrast to the tensor $X^{\left(i_{0}\right)}$ which, in general, is not uniquely determined by $X^{\alpha}$, the covariant representation $X_{\left(i_{\omega}\right)}$ is completely fixed by $X_{\alpha}$. On the other hand, the contravariant representation tensors may be chosen arbitrarily while the covariant $X_{\left(i_{w}\right)}$ are subject to the algebraic condition

$$
X_{\left(i_{w}\right)} \theta\left(i_{0}\right)=0
$$

for all null tensors $\theta^{\left(i_{w}\right)}$. This condition is a consequence of (19.4) and is a necessary and sufficient condition that quantities $X_{\left(i_{w}\right)}$ be the components of a covariant representation tensor.

Similar considerations apply to any vector $Y^{\alpha}$ which lies in $I_{12} \ldots w$. Any such vector has a representation of the form

$$
Y^{\alpha}=\sum_{u=1}^{w} G_{\left(i_{u}\right)}^{\alpha^{\left(i_{s}\right)}}
$$

The $Y^{\left(i_{w}\right)}$ are contravariant surface tensors of order $u$ which are determined except for additive null solutions $\theta^{\left(i_{*}\right)}$ of the equations

$$
G^{\alpha}{ }_{\left(i_{u}\right)} \theta^{\left(i_{u}\right)}=0, \quad \quad u=1,2, \cdots, w .
$$

Indeed each tensor $Y^{\left(i_{u}\right)}$ is readily seen to be the contravariant representation of the projection of $Y^{\alpha}$ in $I_{u}$. In the same way $Y_{\alpha}$ which corresponds to $Y^{\alpha}$ determines the covariant surface tensors $Y_{\left(i_{u}\right)}(u=1,2, \cdots, w)$ by means of the equations

$$
Y_{\left(i_{u}\right)}=Y_{\alpha} G^{\alpha}{ }_{\left(i_{u}\right)},
$$$$
u=1,2, \cdots, w .
$$

As in the contravariant case, $Y_{\left(i_{u}\right)}$ is the covariant representation of the projection of $Y_{\alpha}$ in $I_{u}$.

According to (7.8) and (19.2), the conformal length of $X^{\alpha}$ lying entirely in $I_{w}$, is given by

$$
A_{\alpha \beta} X^{\alpha} X^{\beta}=A_{\alpha \beta} G^{\alpha}{ }_{\left(i_{w}\right)} G^{\alpha}{ }_{\left(h_{w}\right)} X^{\left(i_{w}\right)} X^{\left(h_{w}\right)} .
$$

Consequently, if we use the surface representation $X^{\left(i_{w}\right)}$ of $X^{\alpha}$, conformal length of vectors in $I_{w}$ may be measured by the covariant surface tensor of order $2 w$ whose components are defined by the equations

$$
B_{\left(i_{w}\right) \mid\left(h_{w o}\right)}=A_{\alpha \beta} G^{\alpha}{ }_{\left(i_{w}\right)} G^{\alpha}{ }_{\left(h_{w}\right)} \text {. }
$$

If $w=1, B_{i \mid h}=G_{i h}$. If $w>1$, we call $B_{\left(i_{w}\right) \mid\left(h_{w}\right)}$ the $(w-1)$ th conformal normal measure tensor of $V_{n}$. It is symmetric in the two blocks of indices $\left(i_{w}\right),\left(h_{w}\right)$ 
taken as units. According to (19.6), the conformal normal measure tensors remain unchanged by conformal transformations of $V_{m}$. It follows from (19.2), (19.4) and (19.6) that the contravariant and covariant representations of $X^{\alpha}$ are related by the equations

$$
X_{\left(i_{w}\right)}=B_{\left(i_{w}\right) \mid\left(h_{w}\right)} X^{\left(h_{w}\right)} \text {. }
$$

Similar results may be obtained for vectors $Y^{\alpha}$ in $I_{12} \ldots w$.

From (19.3) and (19.6), it follows that if $\theta^{\left(i_{w}\right)}$ is a solution of (19.3) then

$$
B_{\left(i_{w}\right) \mid\left(h_{w}\right)} \theta^{\left(i_{w}\right)}=0
$$

so that every null solution of (19.3) is also a null solution of (19.7). Conversely, if $\theta^{\left(i_{w}\right)}$ is a null solution of (19.7), it follows from (19.6) and (19.7) that

$$
A_{\alpha \beta}\left[G^{\alpha}{ }_{\left(i_{w}\right)} \theta^{\left(i_{w}\right)}\right]\left[G^{\alpha}{ }_{\left(h_{w}\right)} \theta^{\left(h_{w}\right)}\right]=0 .
$$

Since $A_{\alpha \beta}$ is positive definite, (19.3) is true. Hence the solutions of (19.3) and (19.7) coincide. This means that the ranks of the matrices $\left({ }^{38}\right)$

$$
\left\|G^{\alpha}{ }_{\left(i_{w}\right)}\right\| \text { and }\left\|B_{\left(i_{w}\right) \mid\left(h_{w}\right)}\right\|
$$

are equal. Consequently, the dimension $N_{w}$ of $I_{w}$ is equal to the "rank" of the normal measure tensor $B_{\left(i_{w}\right) \mid\left(h_{w}\right)}$.

We also note that as a consequence of (19.5) and (19.6) and the positive definite character of $A_{\alpha \beta}$,

$$
B_{\left(i_{w}\right) \mid\left(h_{w o}\right)} X^{\left(i_{w}\right)} X^{\left(h_{w}\right)} \geqq 0,
$$

where $X^{\left(i_{w}\right)}$ is any contravariant surface tensor of the $w$ th order. The equality sign holds only if $X^{\left(i_{w}\right)}$ is a null tensor and this is possible only if $w>1$. This means that the conformal measure tensor $G_{i j}$ is positive definite and that the conformal normal measure tensors are positive semi-definite.

The above discussion shows that the conformal measure tensors

$$
B_{i_{1} \mid h_{1}}\left(=G_{\left.i_{1} h_{1}\right)}\right), B_{\left(i_{2}\right) \mid\left(h_{2}\right)}, \cdots, B_{\left(i_{M}\right) \mid\left(h_{M}\right)}
$$

are a system of surface tensors which are used to measure the conformal length of vectors which lie in the conformal osculating or normal vector spaces of $V_{n}$. In a later chapter, we show that if $V_{m}$ is a conformally euclidean space $\bar{R}_{m}$, these conformal measure tensors (19.8) together with the deviation tensor $E_{i n}$ completely determine the subspace $V_{n}$ except for a conformal transformation of $\bar{R}_{m}$.

As in the metric theory, we may base the conformal theory of $V_{n}$ on tensors constructed by symmetrizing the measure tensors. We define the surface tensor $G_{\left(i_{w}\right)\left(h_{w}\right)}(w=1,2, \cdots, M)$ as the tensor of order $2 w$, symmetric in all its $2 w$ indices, which obeys the equations

(38) In the matrix $\left\|B_{\left(i_{w}\right) \mid\left(h_{w}\right)}\right\|$, each row corresponds to a definite sequence of $i$-indices $\left(i_{w}\right)$ and each column to a definite sequence of $h$-indices $\left(h_{w 0}\right)$. 


$$
B_{\left(i_{w}\right)\left(h_{w}\right)} X^{i_{1}} \cdots X^{i_{w}} X^{h_{1}} \cdots X^{h_{w}}=G_{\left(i_{w}\right)\left(h_{w}\right)} X^{i_{1}} \cdots X^{i_{w}} X^{h_{1}} \cdots X^{h_{w}} .
$$

We call the symmetric covariant surface tensors

$$
G_{i_{1} h_{1}}, G_{\left(i_{2}\right)\left(h_{2}\right)}, \cdots, G_{\left(i_{M}\right)\left(h_{M}\right)}, E_{i h}
$$

the first, second, $\cdots, M$ th,$(M+1)$ th conformal fundamental tensors of $V_{n}$ and we name the differential forms

$$
G_{i 1 h_{1}} d x^{i_{1}} d x^{h_{1}}, \cdots, G_{\left(i_{M}\right)\left(h_{M}\right)} d x^{i_{1}} \cdots d x^{i_{M}} d x^{h_{1}} \cdots d x^{h_{M}}, E_{i h} d x^{i} d x^{h}
$$

the first, second, $\cdots$, Mth,$(M+1)$ th conformal fundamental forms of $V_{n}$. A comparison of coefficients in (19.9) shows that

$$
(2 w) ! G_{\left(i_{w}\right)\left(h_{w}\right)}=\sum B_{\left(i_{w}\right) !\left(k_{w}\right)}
$$

where the indices $\left(j_{w}\right),\left(k_{w}\right)$ in the summation assume all possible $(2 w)$ ! permutations of $\left(i_{w}\right),\left(h_{w}\right)$. According to the concluding remarks of $\$ 18$ and the definitions of this section, both the measure tensors and fundamental tensors (except $E_{i h}$ ) exist and are of class $C^{1}$ if the basis of the conformal osculating vector spaces is of class $C^{1}$ in a $V_{n}$ of class $C^{r}$ with $r \geqq 3$. Under the same hypothesis, if $E_{i h}$ exists in $V_{n}$, then all the measure tensors and fundamental tensors also exist in any conformally equivalent subspace $\bar{V}_{n}$. Also, as noted in $\$ 13, E_{\text {ih }}$ exists if (12.23) holds and hence is of class $C^{1}$ if $(14.8)$ is true. We summarize some of the results of this section in the following theorem.

THEOREM 19.1. If the conformal osculating vector spaces of a $V_{n}$ of class $C^{r}$ $(r \geqq 3)$ have a basis of class $C^{1}$ and the deviation tensor $E_{\text {ih }}$ is of class $C^{1}$, then the conformal measure tensors of $V_{n}$ and the conformal fundamental tensors of $V_{n}$ exist and are of class $C^{1}$. If $V_{m} \rightleftarrows \bar{V}_{m}, V_{n} \rightleftarrows \bar{V}_{n}$ by an allowable conformal map, then the conformal measure tensors and conformal fundamental tensors of $\bar{V}_{n}$ exist and, at corresponding points of $V_{n}$ and $\bar{V}_{n}$, the corresponding tensors of $V$. and $\bar{V}_{n}$ are equivalent.

The equivalence of corresponding tensors of $V_{n}$ and $\bar{V}_{n}$ means that these tensors are equal if the parameters on $V_{n}$ and $\bar{V}_{n}$ are chosen so that $x^{i}=\bar{x}^{i}$ at corresponding points.

20. The conformal Frenet equations. We suppose that the inequalities (5.13) hold so that conformal differentiation is possible. In order to derive a system of Frenet equations it is also necessary that the space vectors of the basis (18.11) of the conformal osculating spaces of $V_{n}$ exist and be of class $C^{1}$. As already noted, this last condition is satisfied if (18.12), is true. In what follows, we derive a system of partial differential equations for the quantities

$$
y^{\alpha}\left(x^{i}\right), G_{i_{1}}^{\alpha}, G^{\alpha}{ }_{\left(i_{2}\right)}, \cdots, G^{\alpha}{ }_{\left(i_{M}\right)}, \mu^{\alpha}, \psi, \psi_{i} .
$$

Here $\psi_{i}$ is a symbol for $\partial \psi / \partial x^{i}$. It will be convenient to write these equations so that the left member of each of the equations is a conformal derivative of one of the quantities (20.1). 
According to the definition of $G^{\alpha}{ }_{i_{1}}$ and (8.15), the first two sets of equations may be written as

$$
\begin{aligned}
y^{\alpha}: i_{1} & =G^{\alpha}{ }_{i_{1}}, \\
G^{\alpha}{ }_{i_{1}: i_{2}} & =G^{\alpha}{ }_{\left(i_{2}\right)} .
\end{aligned}
$$

We now derive the wth set of equations of this system. The definition of $G^{\alpha}{ }_{\left(i_{w}\right)}$ implies that it differs from $G^{\alpha_{i_{1}: i_{2}} \ldots i_{w}}$ by a vector in $I_{12} \ldots w-1$, that is, (20.3)

$$
{G^{\alpha}}_{\left(i_{w}\right)}^{\alpha} \equiv G_{i_{1}: i_{2} \cdots i_{w}}^{\alpha_{w}}\left(\bmod I_{12} \cdots w-1\right) \text {. }
$$

By conformal differentiation, we obtain $G^{\alpha}{ }_{\left(i_{w}\right): i_{w}+1} \equiv G_{i_{1}: i_{2}} \ldots i_{w+1}$ (mod $\left.I_{12} \ldots w\right)$. The analogue of $(20.3)$ for $G^{\alpha}{ }_{\left(i_{w}+1\right)}$ permits us to write the last equations as

$$
G^{\alpha}{ }_{\left(i_{w}\right): i_{w+1}} \equiv G^{\alpha}{ }_{\left(i_{w}+1\right)}\left(\bmod I_{12} \ldots{ }_{w}\right) .
$$

These equations may be written as

$$
\begin{aligned}
G^{\alpha}{ }_{\left(i_{w}\right): i_{w+1}}= & G^{\alpha}{ }_{\left(i_{w}+1\right)}+U^{\left(j_{w}\right)}{ }_{\left(i_{w+1}\right)} G^{\alpha}{ }_{\left(j_{w}\right)} \\
& +U^{\left(j_{w-1}\right)}{ }_{\left(i_{w}+1\right)} G_{\left(i_{w}-1\right)}^{\alpha}+\cdots+U^{i_{1}{ }_{\left(i_{w}+1\right)} G^{\alpha}{ }_{j 1} .}
\end{aligned}
$$

Since

$$
A_{\alpha \beta} G^{\alpha}{ }_{\left(i_{w}\right)} G^{\beta}{ }_{\left(h_{v}\right)}=0, \quad w \neq v,
$$

we find, by conformal differentiation, that

$$
A_{\alpha \beta} G^{\beta}{ }_{\left(h_{v}\right)} G^{\alpha}{ }_{\left(i_{w}\right): i_{w}+1}=-A_{\alpha \beta} G^{\alpha}{ }_{\left(i_{w}\right)} G^{\beta}{ }_{\left(h_{v}\right): i_{w+1}} \text {. }
$$

As a consequence of $(20.4), G^{\beta}\left(h_{v}\right): i_{v+1}$ is a vector of $I_{12} \ldots v+1$. Hence if $v=1,2, \cdots, w-2$, it follows from (20.5) that the left member of (20.6), and therefore the right member of (20.6), vanishes. This fact together with (19.6) and (20.4) leads to

$$
U^{\left(h_{v}\right)}{ }_{\left(i_{w}+1\right)} B_{\left(h_{v}\right) \mid\left(k_{v}\right)}=0, \quad v=1,2, \cdots, w-2,
$$

which, according to $\$ 19$, is equivalent to

Hence (20.4) becomes

$$
U_{\left(i_{w}+1\right)}^{\left(h_{v} G_{\left(h_{v}\right)}^{\alpha}\right.}=0, \quad v=1,2, \cdots, w-2 .
$$

$$
\begin{array}{r}
G^{\alpha}{ }_{\left(i_{w}\right): i_{w+1}}=G^{\alpha}{ }_{\left(i_{w}+1\right)}+U^{\left(h_{w}\right)}{ }_{\left(i_{w+1}\right)} G^{\alpha}{ }_{\left(h_{w}\right)}+U^{\left(h_{w-1}\right)}{ }_{\left(i_{w+1}\right)} G^{\alpha}{ }_{\left(h_{w-1}\right)}, \\
w=0,1,2, \cdots, M .
\end{array}
$$

We have included (20.2) and the case $w=M$ in (20.7) by means of the conventions

(20.8) $G_{\left(i_{0}\right)}^{\alpha}=y^{\alpha}, U^{\cdots}{ }_{\left(i_{1}\right)}=U^{\cdots}{ }_{\left(i_{2}\right)}=U^{\cdots_{\left(i_{M+2}\right)}}=U^{\left(i_{M}+1\right)} \ldots=0$,

$$
G^{\alpha}{ }_{\left(i_{M+1}\right)}=0 \text {. }
$$

The last set of these equations is simply (18.10). Equations (20.7) are called 
the conformal Frenet equations of $V_{n}$. It is clear that if these equations exist for $V_{n}$ in $V_{m}$ they are also valid for the $\bar{V}_{n}$ in $\bar{V}_{m}$ obtained by means of any allowable conformal mapping. The quantities $U_{\left(i_{w}+1\right)}^{\left(j_{w}\right)}, U_{\left(i_{w}+1\right)}^{\left(j_{w}-1\right)}$ (determined up to null solutions) are conformal surface tensors since all the other quantities in (20.7) are conformal tensors and the $G^{\alpha}{ }_{\left(i_{w}+1\right)}, G^{\alpha}{ }_{\left(i_{w}\right)}, G^{\alpha}{ }_{\left(i_{w-1}\right)}$ span mutually orthogonal vector spaces. The symmetry properties of the contravariant and covariant indices of these $U$ 's follow from and are similar to those for the $G^{\alpha_{\left(i_{w}\right)}}$ which are derived later. We state some of the results of this section and $\S 18$ in the following theorem.

TheOREM 20.1. Let $V_{n}$ be a subspace of class $C^{r}(r \geqq 3)$ in $a V_{m}$ and let the conformal osculating vector spaces of $V_{n}$ have a basis of class $C^{1}$. Then there exists a set of continuous conformal surface tensors $U_{\left(i_{w}+1\right)}^{\left(h_{w}\right)}, U_{\left(i_{w}+1\right)}^{\left(h_{w}-1\right)}(w=0,1,2, \cdots, M)$ such that the basis beins $G^{\alpha}{ }_{\left.{ }_{i_{w}}\right)}$ satisfy the conformal Frenet equations (20.7) and so that (20.8) is true. If $V_{m} \rightleftarrows \bar{V}_{m}, V_{n} \rightleftarrows \bar{V}_{n}$ by a conformal transformation, then the conformal osculating vector spaces of $\bar{V}_{n}$ exist and have a basis of class $C^{1}$. The corresponding basis beins $\bar{G}_{\left({ }_{\left(i_{w}\right)}\right.}$ for $\bar{V}_{n}$ also satisfy the conformal Frenet equations. At corresponding points of $V_{n}$ and $\bar{V}_{n}$, the respective conformal tangent and normal vector spaces $I_{w}$ and $\bar{I}_{w}$ correspond and their basis beins $G^{\alpha}{ }_{\left({ }_{(}\right)}$and $\bar{G}^{\alpha}{ }_{\left(i_{w}\right)}$ are equivalent tensors.

The equations (20.7) actually involve all of the quantities (20.1). The quantities $\mu^{\alpha}, \psi, \psi_{i}$ which do not appear to be present are implicit in the coefficients of connection $\Gamma^{i}{ }_{j k}, \Gamma_{\beta \gamma}^{\alpha}$ which are used to define the conformal derivatives of the $G^{\alpha}{ }_{\left(i_{w}\right)}$. Consequently we adjoin equations involving the derivatives of $\mu^{\alpha}, \psi, \psi_{i}$ to the conformal Frenet equations. These adjoined equations, which are obtained from (13.14) and (15.4) and are valid if (12.23) is true, are

$$
\begin{aligned}
\psi_{: i}= & \psi_{i} \\
\psi_{i: j}= & \psi_{i} \psi_{j}+E_{i j}-\mu_{\alpha} G^{\alpha}{ }_{i j}+\frac{1}{(m-2)} \rho_{\alpha_{\beta} G{ }_{i} G^{\beta}{ }_{j}} \\
& -\frac{G_{i j}}{2}\left[e^{2 \psi} \mu^{2}+G^{h k} \psi_{h} \psi_{k}+\frac{e^{2 \psi_{\rho}}}{(m-1)(m-2)}\right], \\
\mu_{: i}^{\alpha}= & \frac{e^{-2 \psi}}{(n-1)}\left[G^{\alpha}{ }_{i h: l} G^{h l}+(n-1) G^{\alpha}{ }_{i h} G^{h} \psi_{l}-(n-1) e^{2 \psi} \mu^{\alpha} \psi_{i}\right. \\
& -(n-1) \mu_{\gamma} G^{\gamma_{i h} G^{\alpha} G^{h l}}+G^{\alpha}{ }_{h} K^{h}{ }_{i}+(n-2) G^{\alpha} E^{h}{ }_{i}+G^{\alpha}{ }_{i} E \\
& \left.-C^{\alpha} G_{\beta} G_{i}-\frac{(n-1)}{(m-2)} H^{\alpha \beta} \rho_{\beta \gamma} G^{\gamma}{ }_{i}\right] .
\end{aligned}
$$

Despite the fact that these equations involve metric quantities, their formal structure is unaltered by conformal transformations of $V_{m}$ so that (20.9). are 
conformal equations. Equations (20.7) and (20.9) are a system of partial differential equations in the unknowns (20.1) written in normal form. We call these equations the extended conformal Frenet equations.

Application to hypersurface theory. If $V_{r}$ is a hypersurface of $V_{m}, n=m-1$ and $M=2$. Consequently $I_{2}$ is one-dimensional and $I_{3}$ is empty. Hence we may write $G^{\alpha}{ }_{i j}$ as

$$
G^{\alpha}{ }_{i j}=B_{i j} Z^{\alpha} \text {, }
$$

where $\left.{ }^{39}\right) B_{i j}$ is a symmetric covariant surface tensor and $Z^{\alpha}$ is a space vector which obeys (11.21). Both $B_{i j}$ and $Z^{\alpha}$ are unique except for algebraic sign. Of course, $B_{i j}$ is not a zero tensor since $G^{\alpha}{ }_{i j}$ does not vanish on $V_{n}$. The conformal normal tensor $B_{\left(i_{2}\right) \mid\left(j_{2}\right)}$ of $V_{n}$ is given by

$$
B_{\left(i_{2}\right) \mid\left(j_{2}\right)}=B_{i_{1} i_{2}} \cdot B_{j_{1} j_{2}} \text {. }
$$

We call $B_{i j}$ the conformal normal measure tensor of the hypersurface instead of $B_{\left(i_{2}\right) \mid\left(j_{2}\right)}$ and also use it as the second conformal fundamental tensor of $V_{n}$ instead of the one constructed from $B_{\left(i_{2}\right) \mid\left(j_{2}\right)}$.

In place of (20.7), we may write the conformal Frenet equations of $V_{n}$ as $\left({ }^{40}\right)$

$$
\begin{aligned}
y^{\alpha}: i & =G^{\alpha}, \\
G^{\alpha}{ }_{i: j} & =B_{i j} Z^{\alpha}, \\
Z^{\alpha}: i & =-B_{i}, G^{j k} G^{\alpha}{ }_{k} .
\end{aligned}
$$

To obtain the last set of these equations we note that

$$
Z^{\alpha}: i=G^{\alpha}{ }_{h} U_{i}+Z^{\alpha} U_{i} \text {. }
$$

If we obtain the conformal derivatives of (11.21) and simplify by means of (11.21) and (20.13), we find that

$$
A_{\alpha \beta} Z^{\alpha} Z^{\beta}: i=0, \quad A_{\alpha \beta} Z_{: i}^{\alpha} G^{\beta}{ }_{j}=-B_{i j} .
$$

It follows from (7.7), (11.21), (20.15) and (20.16) that

$$
U_{i}=0, \quad G_{h j} U^{h_{i}}=-B_{i j} .
$$

These equations establish the truth of (20.14). Since $Z^{\alpha}$ is defined algebraically by (11.18), it is of class $C^{r-1}$. According to (20.10), $B_{i j}$ is of class $C^{r-2}$. Hence the conformal Frenet equations of $V_{n},(20.12),(20.13)$ and (20.14), are valid if $r \geqq 2$.

From (20.10), (20.13) and (20.14), we readily find that

$$
G^{\alpha}{ }_{i j: k}=B_{i j: k} Z^{\alpha}-B_{i j} B_{k h} G^{h l} G^{\alpha}{ }_{l}
$$

if $r \geqq 3$. Now, according to (7.12), (20.10) and (20.16),

(39) The tensor $B_{i \mid j}\left(=G_{i j}\right.$ ) is distinguished from $B_{i j}$ by the presence of the vertical bar.

$\left({ }^{10}\right)$ All of these definitions very frequently replace those previously given for any subspace $V_{n}$ if $V_{n}$ is a hypersurface. 


$$
Z^{\alpha}=n^{-1} G^{\sigma_{1} h_{1}} G^{o_{2} h_{2}} B_{\left(o_{2}\right)} G^{\alpha}{ }_{\left(h_{2}\right)} .
$$

If this value for $Z^{\alpha}$ is substituted in (20.17) and the resulting equations compared with (20.7) where $w=2$, we find that

$$
\begin{aligned}
U^{\left(h_{2}\right)}{ }_{\left(i_{3}\right)} & =n^{-1} G^{0_{1} h_{1}} G^{02} h_{2} B_{\left(o_{2}\right)} B_{\left(i_{2}\right): i_{3}}, \\
U^{h_{1}}{ }_{\left(i_{3}\right)} & =-B_{\left(i_{2}\right)} B_{i_{8} h_{2}} G^{\left(h_{2}\right)}
\end{aligned}
$$

for any hypersurface. The $U^{\left(h_{2}\right)}{ }_{\left(t_{2}\right)}$ are determined except for a null solution of $G^{\alpha}{ }_{\left(h_{2}\right)} \theta^{\left(h_{2}\right)}=0$. The components of $U^{\left(h_{1}\right)}{ }_{\left(\xi_{2}\right)}$ are unique.

For a hypersurface, the direction of the unit normal vector $\zeta^{\alpha}$ to $V_{n}$, defined in $\$ 5$, and the direction of the unit conformal normal $Z^{\alpha}$ to $V_{n}$ correspond under any conformal transformation of $V_{m}$. It follows from (7.2) and (13.10) that

$$
Z^{\alpha}=e^{\psi \zeta^{\alpha}} \text {. }
$$

Consequently the mean curvature normal, defined for a hypersurface by (5.11), may be written as

$$
\mu^{\alpha}=e^{-\psi_{\mu} \cdot Z^{\alpha}} .
$$

We define $Z_{\alpha}$ by means of the equations

$$
Z_{\alpha}=A_{\alpha \beta} Z^{\beta}
$$

and multiply (15.3) by $Z_{\alpha}$ and sum for $\alpha$. It follows from (11.21), (20.10), (20.17), (20.20) and (20.21) that

$$
(n-1) \mu_{: i}=e^{-\psi}\left[B_{i h: l} G^{h l}+(n-1) B_{i h} \psi: \imath G^{h l}+\rho^{\alpha}{ }_{\beta \gamma \delta} G^{\beta \delta} G^{\gamma}{ }_{i} Z_{\alpha}\right] .
$$

Since $G^{\beta \delta}=A^{\beta \delta}-Z^{\beta} Z^{\delta}$ and $\rho^{\alpha}{ }_{\beta \gamma \delta} Z_{\alpha} Z^{\beta}=0$, (20.22) becomes

$$
(n-1) \mu_{: i}=e^{-\psi}\left\{G^{h l}\left[B_{i h: l}+(n-1) B_{i n} \psi: l\right]-\rho_{\alpha \beta} Z^{\alpha} G^{\beta}{ }_{i}\right\} .
$$

These equations are valid if $r \geqq 3$. For a hypersurface, the unknowns (20.1) may be replaced by

$$
y^{\alpha}, G^{\alpha}, Z^{\alpha}, \mu, \psi, \psi_{i}
$$

and the last set of equations (20.9) may be replaced by (20.23).

\section{The fundamental equations}

In the metric theory of a hypersurface $V_{m-1}$ in a $V_{m}$, the basic partial differential equations which are satisfied by the coefficients of the first and second fundamental forms of $V_{m-1}$ are the Gauss and Codazzi equations. These equations are the integrability conditions of the system of Frenet equations for the $V_{m-1}$. Much of the geometry of the $V_{m-1}$ is simply a geometric restatement of various analytical consequences of these equations. In the metric theory of any subspace $V_{n}$ in $V_{m}$, a similar but more extensive set of 
fundamental equations is obtained by considering the integrability conditions of the Frenet equations for the $V_{n}$.

In this chapter, we obtain the fundamental equations of the conformal differential geometry of a subspace. These equations involve the various conformal measure and curvature tensors connected with the $V_{n}$ in $V_{m}$ which were defined in earlier sections of the paper. Some of the equations are purely algebraic ones which follow from the definitions and conformal Frenet equations. Others are partial differential equations obtained by a consideration of the integrability conditions of (20.7) and (20.9). Since (20.7) and (20.9) are conformal equations, their integrability conditions are also conformal equations. These latter equations are obtained in a form which explicitly involves only conformal tensors.

These equations include analogues of the classical Gauss-Codazzi equations. However both the algebraic and differential equations which are obtained present several new features. The most important of these differences in the differential equations may be traced to the deviation tensor $E_{i j}$ and equations (20.9) which have no analogue in the metric theory. However, if $n \geqq 4$, the fundamental equations which arise in the metric theory and in the conformal theory of a $V_{n}$ in $V_{m}$ are quite similar. The cases $n=3,2,1$ exhibit increasing degrees of deviation from the "usual" situation.

21. The boundary, algebraic, reality and derived conditions. The inner orientation of the basis beins $G^{\alpha}{ }_{\left({ }_{i w}\right)}$, which determines the various lengths of and angles between these beins, is given by

$$
\begin{gathered}
A_{\alpha \beta} G^{\alpha}{ }_{\left(i_{w}\right)} G^{\beta}{ }_{\left(l_{v}\right)}=0, \quad w \neq v ; w, v=1,2, \cdots, M, \\
A_{\alpha \beta} G^{\alpha}{ }_{\left(i_{w}\right)} G^{\beta}{ }_{\left(l_{w}\right)}=B_{\left(i_{w}\right) \mid\left(l_{w}\right) .}
\end{gathered}
$$

These equations are simply equations (20.5) and (19.5) respectively and are exactly analogous to the corresponding equations in the metric theory. In addition to these equations, we have the equations

$$
A_{a \beta} \mu^{\alpha} G^{\beta}{ }_{j}=0
$$

since the mean curvature normal is orthogonal to the tangent vector space. We think of equations (20.7) and (20.9) as partial differential equations in the unknowns (20.1). Then (21.1), (21.2) and (21.3) are boundary conditions for the system of extended Frenet equations which involve the unknowns $G^{\alpha}{ }_{\left(i_{w}\right)}$ and $\mu^{\alpha}$. We denote (21.1), (21.2) and (21.3) by [B 1 ], [B 2 ] and [ $B 3$ 3 ] respectively and refer to them as the $B$-conditions.

A new type of algebraic condition not occurring in the metric theory is contained in equations (7.11) and (7.12). According to (21.2), (7.12) may be written as

$$
B_{\left(i_{2}\right) \mid\left(h_{2}\right)} G^{i_{1} h_{1}} G^{i_{2} h_{2}}=n .
$$

According to $\$ 19$ and (21.2), equations (7.11) are equivalent to 


$$
B_{\left(i_{2}\right) \mid\left(h_{2}\right)} G^{\left(i_{2}\right)}=0 .
$$

We call equations (21.4) and (21.5) the algebraic conditions or $A$-conditions. We denote the algebraic conditions $(21.5)$ and (21.4) by the symbols $[A 1]$ and $\left[\begin{array}{ll}A & 2\end{array}\right]$ respectively.

Various algebraic conditions may be derived from (21.1) and (21.2). It follows directly from (21.2) and the fact that $A_{\alpha \beta}$ is a symmetric tensor that

$$
B_{\left(i_{w}\right) \mid\left(h_{w}\right)}=B_{\left(h_{w}\right) \mid\left(i_{w}\right)} \text {. }
$$

Also, as was noted in $\$ 19$,

$$
B_{\left(i_{w}\right) \mid\left(h_{w}\right)} \text { is }\left\{\begin{array}{l}
\text { positive definite if } w=1, \\
\text { positive semi-definite if } w>1 .
\end{array}\right.
$$

Furthermore, according to (18.7), (18.8) and (18.9),

$$
\sum_{w=1}^{M} N_{w} \leqq m, \quad N_{w}>0,
$$

where, as noted in $\$ 19, N_{w}$ is the rank of the matrix $\left\|B_{\left(i_{w}\right) \mid\left(h_{w}\right)}\right\|$. Conversely, if tensors $B_{\left(i_{w}\right) \mid\left(h_{w}\right)}$ are defined at a point $P$ of $V_{m}$ such that (21.6), (21.7) and (21.8) are true, then these equations are sufficient conditions for the existence of real basis beins $G^{\alpha}{ }_{\left({ }_{i w}\right)}$ at $P$ which satisfy (21.1) and (21.2). For this reason, we call (21.6), (21.7) and (21.8) the reality conditions or $R$-conditions and denote them by the symbols $[R 1],\left[\begin{array}{ll}R & 2\end{array}\right]$ and $[R 3]$ respectively.

Other equations may be obtained by differentiation of (21.1), (21.2) and (21.3). This differentiation is possible if the hypothesis of Theorem 20.1 is satisfied. If the conformal derivative with respect to $x^{i}$ of $(21.1)$ is found and the result is simplified by means of (20.7) and (21.1) we obtain an identity if $|w-v|>1$. If $v=w+1$, using (21.1) and (21.2) we obtain

(21.9) $\quad B_{\left(i_{w}\right) j \mid\left(l_{w+1}\right)}+U^{\left(h_{w}\right)}{ }_{\left(l_{w+1}\right) j} B_{\left(h_{w}\right) \mid\left(i_{w}\right)}=0, \quad w=1,2, \cdots, M-1$.

In a similar manner, conformal differentiation of (21.2) leads to the result

$$
\begin{array}{r}
B_{\left(i_{w}\right) \mid\left(l_{w}\right): j}-U^{\left(h_{w}\right)}{ }_{\left(i_{w}\right) j} B_{\left(h_{w}\right) \mid\left(l_{w}\right)}-U^{\left(h_{w}\right)}{ }_{\left(l_{w}\right) j} B_{\left(h_{w}\right) \mid\left(i_{w}\right)}=0, \\
w=1,2, \cdots, M .
\end{array}
$$

Also, conformal differentiation of (21.3) and use of the last set of equations (20.9) and of (7.7), (20.7) and (21.1) leads to

$$
U^{k_{i h l} l} G_{k j} G^{h l}+K_{i j}+(n-2) E_{i j}+E G_{i j}-C_{i j}=0 .
$$

In virtue of (21.9) with $w=1$, these conditions are identical with equations (22.10) of the next section. Equations (22.10), in turn, are a consequence of the conformal analogues of the Gauss equations (22.13). Hence the above conditions may be omitted if (22.13) are included as is done later. Equations (21.9) and (21.10) are consequences of the $B$-conditions (21.1) and (21.2). 
We call these equations the derived conditions or simply the $D$-conditions. We denote these equations by the symbols $\left[\begin{array}{ll}D & 1\end{array}\right]$ and $\left[\begin{array}{ll}D & 2\end{array}\right]$ respectively.

We now note several other equations which are consequences of some of the above equations. It follows from (21.5) and (21.9) with $w=2,3, \cdots$, $M-1$ successively that

$$
B_{\left(i_{w}\right) !\left(l_{w}\right)} G^{\left(i_{2}\right)}=0 .
$$

Equations (21.10) and (21.6), (21.9) and (21.10) lead to the equations

$$
\begin{aligned}
& G^{\left(i_{2}\right)} U_{\left(h_{w}\right)}^{\left(i_{w+1}\right)} B_{\left(h_{w}\right) \mid\left(l_{w}\right)}=0, \quad w=2,3, \cdots, M, \\
& G^{\left(i_{2}\right)} U^{\left(h_{w-1}\right)}{ }_{\left(i_{w+1}\right)} B_{\left(h_{w-1}\right) \mid\left(l_{w-1}\right)}=0 .
\end{aligned}
$$

Equations similar to (21.11) and (21.12) may also be obtained by conformal differentiation of (21.6).

Application to hypersurface theory. If $V_{n}$ is a hypersurface, $n=m-1$ and $M=2$. In this case, we write the conformal Frenet equations as (20.12), (20.13) and (20.14) and we replace the last set of equations (20.9) by (20.23). We also eliminate the tensor $B_{\left(i_{2}\right)\left(j_{2}\right)}$ by means of equations (20.11).

It is easy to see that the boundary conditions (21.1) may be replaced by the first set of equations (11.21)

$$
A_{\alpha \beta} Z^{\alpha} G^{\beta}{ }_{j}=0
$$

and that (21.2) may be written as (7.7) and the second set of equations (11.21)

$$
\begin{aligned}
A_{\alpha \beta} G^{\alpha}{ }_{i} G^{\beta} & =G_{i j}, \\
A_{\alpha \beta} Z^{\alpha} Z^{\beta} & =1 .
\end{aligned}
$$

We denote these conditions which are equivalent to (21.1) and (21.2) by the symbols $\left[B^{\prime} 1\right]$ and $\left[B^{\prime} 2\right]$ respectively. Since $\mu^{\alpha}$ is replaced by $e^{\psi} \mu Z^{\alpha},(21.3)$ is a consequence of $\left[B^{\prime} 1\right]$ and may be omitted for a hypersurface. Equations (21.4) and (21.5) become

$$
\begin{aligned}
B_{\left(i_{2}\right)} B_{\left(h_{2}\right)} G^{i_{1} h_{1}} G^{i_{2} h_{2}} & =n, \\
B_{\left(i_{2}\right)} G^{\left(i_{2}\right)} & =0 .
\end{aligned}
$$

We denote (21.14) and (21.13) by the symbols $\left[A^{\prime} 1\right]$ and $\left[A^{\prime} 2\right]$ respectively. We also note that (21.6) and (21.7) need only be stated for the case $w=1$; namely,

$$
G_{i j}=G_{j i}
$$

$G_{i j}$ is fositive definite.

The remaining case, $w=2$, follows directly from (20.11). We denote (21.15) and (21.16) by the symbols $\left[R^{\prime} 1\right]$ and $\left[R^{\prime} 2\right]$ respectively. Equation (21.8) is always true since $M=2$ and $N_{1}=n, N_{2}=1$ follows from (21.16) and (20.11) 
respectively. Finally, the derived conditions obtained by differentiating $\left[B^{\prime} 1\right]$ and $\left[B^{\prime} 2\right]$ are true identically in virtue of the conformal Frenet equations and $B$-conditions. Hence there are no conditions corresponding to $[D 1]$ and $\left[\begin{array}{ll}D & 2]\end{array}\right]$ for a hypersurface.

22. The conformal Gauss-Codazzi equations. Each of the various conformal Frenet equations contains a single contravariant space index $\alpha$. Consequently the integrability conditions of these equations also contain $\alpha$ as the only space index. Therefore if the integrability conditions for the Frenet equations whose left members are $G^{\alpha}{ }_{\left(i_{w}\right): i_{w+1}}(w=0,1,2, \cdots, M)$ are multiplied by $A_{\alpha \beta} G^{\beta}{ }_{\left(l_{v}\right)}(\imath=1,2, \cdots, M, \infty)$ and summed for $\alpha, \beta$ the resulting equations only involve surface indices. We denote these equations by the symbol $[w, v]$. It is clear that the integrability conditions of the conformal Frenet equations and the equations $[w, v]$ where $w=0,1,2, \cdots, M$; $v=1,2, \cdots, M, \infty$ are equivalent.

The integrability conditions of the first set of the conformal Frenet equations, which describe the variation of $y^{\alpha}\left(=G^{\alpha}{ }_{\left(i_{0}\right)}\right)$, are $y^{\alpha}:(j k)^{-}=0$. According to the first two sets (20.2) of the conformal Frenet equations, the above equations become $G^{\alpha}{ }_{(j k)^{-}}=0$ which, in turn, are equivalent to

$$
A_{\alpha \beta} G^{\alpha}{ }_{(j k)}-G^{\beta}{ }_{\left(l_{v}\right)}=0,
$$$$
v=1,2, \cdots, M, \infty \text {. }
$$

These are conditions $[0, v]$. As a result of (21.1) and (21.2) all of these equations are identities except for the condition $[0,2]$ which is

$$
B_{(j k)-\mid\left(l_{2}\right)}=0 \text {. }
$$

This is equivalent to the symmetry property of $G^{\alpha}{ }_{j k}$ in its covariant indices already noted at the end of $\S 8$.

The integrability conditions of the second set of conformal Frenet equations are

$$
G^{\alpha}{ }_{i:(j k)^{-}}=G^{\alpha}{ }_{0} K^{o_{i j k}}-G^{\beta}{ }_{i} R^{\alpha}{ }_{\beta j k}
$$

according to (17.1). The tensor $R^{\alpha}{ }_{\beta j k}$ is defined by (16.8). Now from (20.7) and (20.8), we obtain $G^{\alpha}{ }_{i: j k}=G^{\alpha}{ }_{i j: k}=G^{\alpha}{ }_{i j k}+U^{\left(h_{2}\right)}{ }_{i j k} G^{\alpha}{ }_{\left(h_{2}\right)}+U^{h_{1}{ }_{i j k}} G^{\alpha}{ }_{h_{1}}$. These last equations show that (22.3) is equivalent to

$$
\begin{aligned}
& A_{\alpha \beta} G^{\beta}{ }_{\left(l_{v}\right)}\left[G^{\alpha}{ }_{i(j k)}-+U^{\left(h_{2}\right)}{ }_{i(j k)}-G^{\alpha}{ }_{\left(h_{2}\right)}+U^{h_{1}}{ }_{i(j k)}-G^{\alpha}{ }_{h_{1}}\right] \\
& =A_{\alpha \beta} G^{\alpha}{ }_{0} G^{\beta}{ }_{\left(l_{v}\right)} K^{o}{ }_{i j k}-A_{\alpha \varepsilon} G^{\mathbf{e}}{ }_{\left(l_{v}\right)} G^{\beta}{ }_{i} R^{\alpha}{ }_{\beta j k} \text {. }
\end{aligned}
$$

These are conditions $[1, v]$. According to (21.1) and (21.2), the first of these conditions, when $v=1$, becomes

$$
U^{h_{1}(j k)}-G_{h_{1} l_{1}}=K_{l_{1 i} i k}-R_{\alpha \beta j k} G_{l_{1}}^{\alpha} G_{i}^{\beta} .
$$

Now (21.9) with $w=1$ may be written as

$$
U^{h_{1}{ }_{i j k}} G_{h_{1} l_{1}}=-B_{i j \mid l_{1} k} \text {. }
$$


If we write $h$ for $l_{1}$ and take account of (22.6), (22.5) may be written as

$$
B_{i(k \mid j)^{-} h}=K_{h i j k}-R_{\alpha \beta j k} G^{\alpha} G^{\beta}{ }_{i} .
$$

These are conformal analogues of the Gauss equations which we now proceed to simplify.

From these equations and (7.4), (7.7), (14.2), (14.3), (14.6), (16.8), (16.16), (20.7), (21.4), (21.5) and (22.6), we find by an easy calculation that

$$
\begin{aligned}
(n-1) B_{i k \mid h j} G^{h k}= & K_{i j}-C_{i j}+(n-2) E_{i j}+(n-2) B_{i k \mid h j} G^{h k} \\
& +[C+(3-2 n) E-K+n] G_{i j} .
\end{aligned}
$$

If these equations are multiplied by $G^{i j}$ and summed,

$$
K=n+C+2(1-n) E .
$$

Substitution of this result in (22.8) yields

$$
K_{i j}=B_{i k \mid h j} G^{h k}+C_{i j}+(2-n) E_{i j}-E G_{i j} .
$$

We replace $K_{i j}$ in (16.8) by its value given in (22.10) and simplify the resulting equations, making use of the conformal analogue of (4.12) and of

$$
H^{\alpha}{ }_{\gamma} G^{\gamma_{i j: k}}=G^{\alpha}{ }_{i j k}+U^{\left(h_{2}\right)}{ }_{i j k} G^{\alpha}{ }_{\left(h_{2}\right)}=A^{\alpha}{ }_{\gamma} G^{\gamma_{i j: k}}+G^{h \alpha} B_{i j \mid k h}
$$

which follows from (20.7) and (22.6). Then equations (16.8) become equations (16.9).

If we use this simplified form of $R^{\alpha}{ }_{\beta j k}$ in (22.7), we find that (22.7) becomes

$$
B_{i(k \mid j)^{-h}}=K_{h i j k}-R_{h i j k}
$$

where $R_{h i j k}$ is given by (16.11). Conversely, it is easy to see that (22.7) is an identity in virtue of (22.12) and that (16.8) and (16.9) are equivalent if (22.12) holds. Consequently condition $[1,1]$, originally written as (22.5), may also be written as (22.12). According to (22.12) and (16.11), these equations are

$$
B_{i(k \mid j)^{-h}}=K_{h i j k}-C_{h i j k}+G_{i(j} E_{k)^{-} h}+G_{h(k} E_{i)^{-i}} .
$$

We call (22.13) the conformal Gauss equations of the $V_{n}$ in $V_{m}$. If these equations are multiplied by $G^{h k}$ and then by $G^{i j}$ we obtain equations (22.10) and (22.9) respectively after summation on repeated indices.

A comparison of the metric Gauss equations (5.16) and (22.12) exhibits an exact analogy if the analogous pairs of metric and conformal tensors are: $b_{i k \mid j l}, B_{i k \mid j l} ; \kappa_{h i j k}, K_{h i j k} ; \rho_{h i j k}, R_{h i j k}$. However, if this last pair is replaced by $\rho_{h i j k}, C_{h i j k}$, (22.13) shows that an additional expression involving $E_{i j}$ is present in the conformal Gauss equations. This additional expression is responsible for many of the new geometric features in the conformal theory of $V_{n}$ which are absent in the metric theory.

Now $K_{h i j k}$ and $\kappa_{h i j k}$ are the Riemann curvature tensors formed with re- 
spect to $G_{i j}$ and. $g_{i j}$ respectively. Since, from (7.3), the tensors $G_{i j}$, $g_{i j}$ are in conformal correspondence, it follows from $\$ 11$ that, if $n>2, \uparrow K^{h_{i j k}}=\uparrow \kappa^{k}{ }_{i j k}$. In these equations, the $\uparrow$ operation for the left member is with respect to $G_{i j}$ while that for the right member is with respect to $g_{i j}$. As a result of (11.26), (11.28) and (11.29),

$$
\uparrow K_{h i j k}=W_{h i j k} .
$$

Consequently, if $n>2$ and the $\uparrow$ operation with respect to $G_{i j}$ is applied to (22.13), we find that

$$
W_{h i j k}=\uparrow B_{h i j k}+\uparrow C_{h i j k}
$$

after use is made of (11.30) and (11.31). The tensor $\uparrow C_{h i j k}$ is defined by (16.14) and $\uparrow \mathscr{B}_{h i j k}$, defined by (11.28), is

$(22.16)$

$$
\uparrow \mathscr{B}_{h i j k}=\mathscr{B}_{h i j k}+\frac{1}{(n-2)}\left[G_{h(j} \mathbb{B}_{k)^{-i}}+G_{i(k} \mathcal{B}_{j)^{-h}}\right]
$$

where we have written

$$
+\frac{\mathbb{B}}{(n-1)(n-2)} G_{h(k} G_{j)-i}
$$

$$
\mathbb{B}_{h i j k}=B_{i(k \mid j)^{-h}}, \quad \mathbb{B}_{i j}=\mathscr{B}_{h i j k} G^{h k}, \quad \mathbb{B}=\mathscr{B}_{i j} G^{i j} .
$$

It follows from (21.6) and (21.7) that

$$
\mathscr{B}_{i j}=B_{i k \mid h j} G^{h k}, \quad \mathscr{B}=n .
$$

We return to conditions $[1, v]$ or $(22.4)$ and let $v=2$. If the resulting equations are simplified by means of (16.4), (21.1) and (21.2) they become

$$
U^{\left(h_{2}\right)_{i(j k)}}-B_{\left(h_{2}\right) \mid\left(l_{2}\right)}=-A_{\alpha \beta} R^{\alpha}{ }_{i j k} G^{\beta}{ }_{\left(l_{2}\right)} .
$$

Use of (11.16), (16.10), (21.1), (21.2) and (22.11) transforms (22.19) into the equations

$$
U^{\left(h_{2}\right)}{ }_{i(j k)}-B_{\left(h_{2}\right) \mid\left(l_{2}\right)}=-D_{\beta i j k} G^{\beta}{ }_{\left(l_{2}\right)}
$$

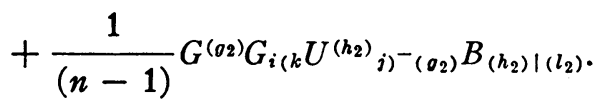

This is condition $[1,2]$. Equations (22.20) are called the conformal Codazzi equations of the $V_{n}$ in $V_{m}$.

If $M$ exceeds 2 , we let $v$ equal 3 in the conditions $[1, v]$ and simplify as was done to obtain (22.19). We obtain

$$
B_{i(j k)-\mid\left(l_{3}\right)}=-A_{\alpha \beta} R^{\alpha}{ }_{i j k} G^{\beta}{ }_{\left(l_{3}\right)} .
$$

We proceed as in the derivation of (22.20) and find that the above equations are equivalent to 
(22.22) $\quad B_{i(j k)-\mid\left(l_{3}\right)}=-D_{\beta i j k} G^{\beta}{\left(l_{3}\right)}+\frac{1}{(n-1)} G^{\left(a_{2}\right)} G_{i(k} B_{j)}-\left(o_{2}\right) \mid\left(l_{3}\right)$.

This is condition. $[1,3]$. The cases where $v>3$ are considered in a similar manner and lead to the following analogues of (22.21) and (22.22):

$$
\begin{aligned}
A_{\alpha \beta} R^{\alpha}{ }_{i j k} G^{\beta}{ }_{\left(l_{u}\right)} & =0, & & u=4,5, \cdots, M, \infty, \\
D_{\beta i j k} G^{\beta}{ }_{\left(l_{k}\right)} & =0, & & u=4,5, \cdots, M, \infty .
\end{aligned}
$$

The latter of these is condition $[1, u], u>3$.

23. Higher integrability conditions. The third set $(w=2)$ of the conformal Frenet equations is

$$
G^{\alpha}{ }_{\left(i_{2}\right): j}=G^{\alpha}{ }_{\left(i_{2}\right) j}+U^{\left(h_{2}\right)}{ }_{\left(i_{2}\right) j} G^{\alpha}{ }_{\left(h_{2}\right)}+U^{h_{1}}{ }_{\left(i_{2}\right) j} G^{\alpha}{ }_{h_{1}} \text {. }
$$

The integrability conditions of these equations are

$$
G^{\alpha}{ }_{\left(i_{2}\right):(j k)^{-}}=G^{\alpha}{ }_{\rho_{i 2}} K^{o_{i_{1} j k}}+G^{\alpha_{i_{1} 0}} K^{o_{i_{2} j k}}-G^{\beta}{ }_{\left(i_{2}\right)} R^{\alpha}{ }_{\beta j k} .
$$

Conformal differentiation of (23.1) and simplification by means of (20.7) and (20.8) gives

$$
\begin{aligned}
& G^{\alpha}{ }_{\left(i_{2}\right): j k}=G^{\alpha}{ }_{\left(i_{2}\right) j k}+\left[U^{\left(h_{3}\right)}{ }_{\left(i_{2}\right) j k}+U^{\left(h_{22}{ }_{\left(i_{2}\right)}{ }_{j} G^{h_{3}}{ }_{k}\right] G^{\alpha}{ }_{\left(h_{3}\right)}}\right. \\
& +\left[U^{\left(h_{2}\right)}{ }_{\left(i_{2}\right) j k}+U^{\left(h_{2}\right)}{ }_{\left(i_{2}\right) j: k}+U^{\left(o_{2}\right)}{ }_{\left(i_{2}\right) j} U^{\left(h_{2}\right)}{ }_{\left(o_{2}\right) k}+U^{\left.h_{1}{ }_{\left(i_{2}\right) j} G^{h_{2}}{ }_{k}\right] G^{\alpha}{ }_{\left(h_{2}\right)}}\right. \\
& +\left[U^{h_{1}}{ }_{\left(i_{2}\right) j: k}+U^{\left(o_{2}\right)}{ }_{\left(i_{2}\right) j} U^{h_{1}}{ }_{\left(o_{2}\right) k}\right] G^{\alpha}{ }_{h_{1}} \text {. }
\end{aligned}
$$

Also, from (16.9), (20.7), (20.8), (21.1) and (21.2),

(23.4) $G^{\beta}{ }_{\left(i_{2}\right)} R^{\alpha}{ }_{\beta j k}=D^{\alpha_{\beta j k}} G^{\beta}{ }_{\left(i_{2}\right)}+\frac{1}{(n-1)} G^{\left(o_{2}\right)} G^{\alpha}{ }_{(k} U^{\left(h_{2}\right)}{ }_{j)^{-}}{ }_{\left(o_{2}\right)} B_{\left(h_{2}\right) \mid\left(i_{2}\right)}$.

We substitute (23.3) and (23.4) in (23.2) and multiply successively by $A_{\alpha \beta} G^{\beta}{ }_{\left(l_{v}\right)}(v=1,2, \cdots, M, \infty)$ and simplify using (18.13), (21.1) and (21.2). This gives the various integrability conditions $[2, v]$ :

$$
\begin{aligned}
& {\left[U^{h}{ }_{\left(i_{2}\right)(j: k)^{-}}+U^{\left(0_{2}\right)}{ }_{\left(i_{2}\right) j^{-}}-U^{h}{ }_{\left(o_{2}\right) k^{-}}\right] G_{h l}=-D_{\alpha \beta j k} G^{\alpha}{ }_{l} G^{\beta}{ }_{\left(i_{2}\right)}} \\
& +\frac{1}{(n-1)} G^{\left(g_{2}\right)} G_{l(j} U_{k)^{\left(h_{2}\right)}-\left(o_{2}\right)} B_{\left(h_{2}\right) \mid\left(i_{2}\right)}, \\
& {\left[U_{\left(h_{2}\right)}^{\left(i_{2}\right)(j k)^{-}}+U_{\left(h_{2}\right)}^{\left(i_{2}\right)(j: k)^{-}}+U^{\left(o_{2}\right)}{ }_{\left(i_{2}\right)}, U^{\left(h_{2}\right)}{ }_{\left(o_{2}\right) k^{-}}\right.} \\
& \left.+U_{\left.\left(i_{2}\right)\left({ }_{j} G^{h_{2}}\right)^{-}\right]}\right] B_{\left(h_{2}\right) \mid\left(l_{2}\right)} \\
& =B_{o i_{2} \mid\left(l_{2}\right)} K^{\theta_{i_{1} j k}}+B_{i_{1} \mid\left(l_{2}\right)} K^{o}{ }_{i_{2} j k}-D_{\alpha \beta j k} G^{\alpha}{ }_{\left(l_{2}\right)} G^{\beta}{ }_{\left(i_{2}\right)} \text {, } \\
& \text { (23.7) }\left(U^{\left(h_{3}\right)}{ }_{\left(i_{2}\right)(j k)^{-}}+U^{\left(h_{2}\right)}{ }_{{ }_{\left(i_{2}\right)(j} G^{h_{8}}{ }_{k}-}\right] B_{\left(h_{3}\right) \mid\left(l_{3}\right)}=-D_{\alpha \beta j k} G^{\alpha}{ }_{\left(l_{3}\right)} G^{\beta}{ }_{\left(i_{2}\right)} \text {, } \\
& B_{\left(i_{2}\right)(j k)}-\mid\left(l_{4}\right)=-D_{\alpha \beta j k} G^{\alpha}{ }_{\left(l_{1}\right)} G^{\beta}{ }_{\left(i_{2}\right)} \text {, } \\
& 0=D_{\alpha \beta j k} G^{\alpha}{ }_{\left(l_{u}\right)} G^{\beta}{ }_{\left(i_{2}\right)}, \quad u=5,6, \cdots, M, \infty .
\end{aligned}
$$

These are conditions $[2,1],[2,2],[2,3],[2,4]$ and $[2, u], u>4$, respec- 
tively. In (23.5), we have omitted the subscript 1 from the indices $h, l$.

We now consider the general $(w+1)$ th set of conformal Frenet equations (20.7). The integrability conditions of this set of equations are

$$
G_{\left({ }_{\left.i_{w}\right):(j k)}-\right.}^{\alpha}=\sum_{u=1}^{w} G^{\alpha}{ }_{\left(i_{u-1}\right) g i_{u+1}} \cdots_{i_{w}} K^{o_{i_{u} j k}}-G^{\beta}{ }_{\left(i_{w w}\right)} R^{\alpha}{ }_{\beta j k \text {. }}
$$

Now conformal differentiation of (20.7) and simplification by means of the conformal Frenet equations leads to

$$
\begin{aligned}
& G^{\alpha}{ }_{\left(i_{w}\right): j k}=G^{\alpha}{ }_{\left(i_{w}\right) j k}+\left[U^{\left(h_{w+1}\right)}{ }_{\left(i_{w}\right) j k}+U^{\left(h_{w}\right)}{ }_{\left(i_{w}\right) j} G^{h_{w+1}}{ }_{k}\right] G^{\alpha}{ }_{\left(h_{w+1}\right)}
\end{aligned}
$$

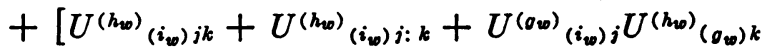

$$
\begin{aligned}
& \left.+U^{\left(h_{w 0-1}\right)}{ }_{\left(i_{w}\right) ;} ; G^{h_{0}} k\right] G^{\alpha}{ }_{\left(h_{w}\right)} \\
& +\left[U^{\left(h_{0-1}\right)}{ }_{\left(i_{w}\right) j: k}+U^{\left(g_{w}\right)}{ }_{\left(i_{w}\right) j} U^{\left(h_{w-1}\right)}{ }_{\left(o_{w}\right) k}\right. \\
& \left.+U^{\left(\sigma_{w-1}\right)}{ }_{\left(i_{w}\right) j} U^{\left(h_{w-1}\right)}{ }_{\left(\sigma_{w-1}\right) k}\right] G^{\alpha}{ }_{\left(h_{w}-1\right)} \\
& +U^{\left(o_{w-1}\right)}{ }_{\left(i_{w}\right) j} U^{\left(h_{w-2}\right)}{ }_{\left(g_{w-1}\right) k} G^{\alpha}{ }_{\left(h_{w-2}\right)} \text {. }
\end{aligned}
$$

Also, from (16.9), (20.7), (20.8), (21.1) and (21.2), we find that

$$
\begin{aligned}
& G^{\beta}{ }_{\left(i_{8}\right)} R^{\alpha}{ }_{\beta j k}=D^{\alpha}{ }_{\beta j k} G^{\beta}{ }_{\left(i_{8}\right)}+\frac{1}{(n-1)} G^{\left(o_{2}\right)} G^{\alpha}{ }_{(k} B_{j)^{-}}\left(o_{2}\right) \mid\left(l_{3}\right), \\
& G^{\beta}{ }_{\left(i_{u}\right)} R^{\alpha}{ }_{\beta j k}=D^{\alpha}{ }_{\beta j k} G^{\beta}{ }_{\left(i_{k}\right)}, \quad u=4,5, \cdots, M, \infty .
\end{aligned}
$$

We substitute (23.11) and (23.12) in (23.10) and multiply successively by $A_{\alpha \beta} G^{\beta}{ }_{\left(l_{v}\right)}(v=1,2, \cdots, M, \infty)$ and then simplify using (18.13), (21.1) and (21.2). In this manner we obtain the integrability conditions [w, $v]$ for $w>2$. These equations are

$$
\begin{aligned}
& U^{\left(o_{2}\right)}{ }_{\left(i_{3}\right) j}-U^{h}{ }_{\left(o_{2}\right) k}-G_{h l}=-D_{\alpha \beta j k} G^{\alpha}{ }_{l} G^{\beta}{ }_{\left(i_{3}\right)}+\frac{1}{(n-1)} G^{\left(o_{2}\right)} G_{l(j} B_{k)^{-}\left(o_{2}\right) \mid\left(l_{3}\right)}, \\
& U^{\left(o_{w-1}\right)}{ }_{\left(i_{w}\right)}, U^{\left(h_{w-2}\right)}{ }_{\left(o_{w-1}\right) k}-B_{\left(h_{w-2}\right) \mid\left(l_{w-2}\right)}=-D_{\alpha \beta j k} G^{\alpha}{ }_{\left(l_{w-2}\right)} G^{\beta}{ }_{\left(i_{w}\right)}, \\
& {\left[U^{\left(h_{w-1}\right)}{ }_{\left(i_{w}\right)(j: k)^{-}}+U^{\left(o_{w}\right)}{ }_{\left(i_{w}\right)} j^{-} U^{\left(h_{w-1}\right)}{ }_{\left(O_{w}\right) k^{-}}\right.} \\
& \left.+U^{\left(o_{w-1}\right)}{ }_{\left(i_{w}\right)}-U^{\left(h_{w-1}\right)}{ }_{\left(\theta_{w-1}\right) k^{-}}\right] B_{\left(h_{w-1}\right) \mid\left(l_{w-1}\right)}=-D_{\alpha \beta j k} G^{\alpha}{ }_{\left(l_{w-1}\right)} G^{\beta}{ }_{\left(i_{w}\right)}, \\
& {\left[U^{\left(h_{w}\right)}{ }_{\left(i_{w}\right)(j k)^{-}}+U^{\left(h_{w}\right)}{ }_{\left(i_{w}\right)(j: k)^{-}}+U^{\left(g_{w}\right)}{ }_{\left(i_{w}\right)} j^{-} U^{\left(h_{w}\right)}{ }_{\left(\sigma_{w}\right) k^{-}}\right.}
\end{aligned}
$$

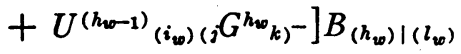

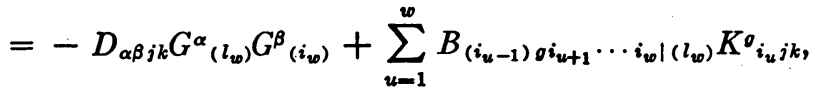

$$
\begin{aligned}
& {\left[U^{\left(h_{w+1}\right)}{ }_{\left(i_{w}\right)(j k)}+U^{\left(h_{w}\right)}{ }_{\left(i_{w}\right)(j} G^{h_{w+1}}{ }_{k)}-\right] B_{\left(h_{w}+1\right) \mid\left(l_{w+1}\right)}} \\
& =-D_{\alpha \beta j k} G^{\alpha}{ }_{\left(l_{w+1}\right)} G^{\beta}{ }_{\left(i_{v}\right)}, \\
& B_{\left(i_{w}\right)(j k)-\mid\left(l_{w+2}\right)}=-D_{\alpha \beta j k} G^{\alpha}{ }_{\left(l_{w}+2\right)} G^{\beta}{ }_{\left(i_{w}\right)},
\end{aligned}
$$




$$
0=D_{\alpha \beta j k} G^{\alpha}{ }_{\left(l_{u}\right)} G^{\beta}{ }_{\left(i_{w}\right)}, \quad u>w+2 \text { or } u<w-2 .
$$

The above equations are conditions $[3,1],[w, w-2]$ with $w>3,[w, w-1]$, $[w, w],[w, w+1],[w, w+2]$ and $[w, u]$ with $u>w+2$ or $u<w-2$ respectively. In all of these equations, it is assumed that $w>2$ and that $w$ does not exceed $M$ while $u$ has the range $1,2, \cdots, M, \infty$. In accordance with (20.8), $U_{\ldots}^{\left(h_{M}+1\right)}, U_{\left({ }_{M+2}\right)}^{\cdots}, B_{\left(h_{M+1}\right) \mid\left(l_{M+1}\right)}$ and $B_{\left(h_{M+2}\right) \mid\left(l_{M+2}\right)}$ are zero tensors and the appropriate substitution should be made for these tensors in the above equations wherever they occur.

In the above paragraphs and in the previous section, we have derived the various integrability conditions $[w, v]$ of the conformal Frenet equations. However, not all of these conditions are distinct. In the sequel, we indicate which of the $[w, v]$ may be omitted because of the duplications which arise.

If neither $w$ nor $v$ exceeds $M$, a straightforward calculation shows that

$$
\left[A_{\alpha \beta} G^{\alpha}{ }_{\left(i_{w}\right)} G^{\beta}{ }_{\left(l_{v}\right)}\right]_{:(j k)^{-}}=A_{\alpha \beta} G^{\alpha}{ }_{\left(i_{w}\right)}:(j k)-G^{\beta}{ }_{\left(l_{v}\right)}-A_{\alpha \beta} G^{\alpha}{ }_{\left(i_{w}\right)} G^{\beta}{ }_{\left(l_{v}\right):(j k)^{-}} .
$$

If $w \neq v$, it follows from (21.1) that the left member of these equations is zero. Consequently (23.20) may be written as $A_{\alpha \beta} G^{\alpha}{ }_{\left(i_{w}\right):(j k)}-G^{\beta}{ }_{\left(l_{v}\right)}+R_{\alpha \beta j k} G^{\alpha}{ }_{\left(l_{v}\right)} G^{\beta}{ }_{\left(i_{w}\right)}$ $=-A_{\alpha \beta} G^{\alpha}{ }_{\left(l_{v}\right):(j k)}-G^{\beta}{ }_{\left(i_{w}\right)}-R_{\alpha \beta j k} G^{\alpha}{ }_{\left(i_{w}\right)} G^{\beta}{ }_{\left(l_{v}\right)}, v \neq w$. According to (23.10), the left member of these equations equated to zero is condition $[w, v]$ and the right member equated to zero is condition $[v, w]$. Hence it follows that conditions $[w, v]$ and $[v, w]$ are identical. This means that all the integrability conditions of the conformal Frenet equations are conditions $[w, v]$ with $v \geqq w$.

It is easy to see that the differentiation implicit in the integrability conditions $[w, v]$ is possible if $V_{n}$ is of class $C^{r}$ with $r \geqq 4$ and if the basis beins (18.11) of $V_{n}$ form a basis of class $C^{2}$. Comparison with (18.12) shows that

$$
r \geqq M+2
$$

is a sufficient but not necessary condition for the satisfaction of these last conditions.

Finally, we note that the extended conformal Frenet equations (20.7) and (20.9) and the $B$-conditions (21.1) and (21.2) remain unchanged if any tensor $U^{\left(h_{w}\right)}{ }_{\left(i_{w+1}\right)}, U^{\left(h_{w-1}\right)}{ }_{\left(i_{w+1}\right)}$ is replaced by another which differs from it by an additive null solution. Consequently any equations derived from (20.7), (20.9), (21.1) and (21.2) such as the $D$-conditions and the integrability conditions also must remain unchanged if any $U$ is changed by an additive null tensor. This fact may be verified directly by using various relationships which are obeyed by null tensors.

As an illustration, we derive and use some of these relationships. By conformal differentiation of (19.3) with respect to $x^{i}$ and use of (20.7), we obtain

$$
G^{\alpha}{ }_{\left(i_{w}\right) j} \theta^{\left(i_{w}\right)}+G^{\alpha}{ }_{\left(h_{w}\right)}\left[\theta^{\left(h_{w}\right)}: j+U^{\left(h_{w}\right)}{ }_{\left(i_{w}\right) j} \theta^{\left(i_{w}\right)}\right]+G^{\alpha}{ }_{\left(h_{w-1}\right)} U^{\left(h_{w-1}\right)}{ }_{\left(i_{w}\right) ;} \theta^{\left(i_{w}\right)}=0 .
$$


It follows from these equations and (21.1) and (21.2) that the relationships

$$
\begin{aligned}
B_{\left(i_{w}\right) j \mid\left(l_{w+1}\right)} \theta^{\left(i_{w}\right)} & =0, \\
B_{\left(h_{w}\right) \mid\left(l_{w}\right)}\left[\theta^{\left(h_{w}\right)}:_{i}+U^{\left(h_{w}\right)}{ }_{\left(i_{w}\right) j} \theta^{\left(i_{w}\right)}\right] & =0, \\
B_{\left(h_{w-1}\right) \mid\left(l_{w-1}\right)} U^{\left(h_{w-1}\right)}{ }_{\left(i_{w}\right) j} \theta^{\left(i_{w}\right)} & =0
\end{aligned}
$$

are true if $\theta^{(i w)}$ is a null tensor satisfying (19.3). An example of the application which may be made of these relationships follows. If $U^{\left(h_{w}+1\right)}{ }_{\left(i_{w}\right) j k}$ in (23.17) is changed by an additive null tensor $\theta^{\left(h_{w}+1\right)}$, the additional terms $\theta^{\left(h_{w}+1\right)} B_{\left(h_{w+1}\right) \mid\left(l_{w+1}\right)}$ must vanish by definition of $\theta^{\left(h_{w}+1\right)}$. If $U^{\left(h_{w}\right)}{ }_{\left(i_{w}\right) j}$ in $(23.17)$ is changed by a null solution $\theta^{\left(h_{w}\right)}$, the additional terms $\theta^{\left(h_{w}\right)} G^{\left(h_{w}+1\right)}{ }_{k} B_{\left(h_{w+1}\right) !\left(l_{w+1}\right)}$ must vanish according to the first of the above relationships. Hence (23.17) remains unchanged.

Application to hypersurface theory. If $V_{n}$ is a hypersurface, the integrability conditions $[w, v]$ may be derived more easily from $(20.12),(20.13)$ and (20.14) instead of from (20.7). Of the integrability conditions, $[0,1]$ is a consequence of (11.21) and $[0,2],[1,1]$ and $[1,2]$ may be written as

$$
\begin{aligned}
B_{(j k)^{-}} & =0, \\
B_{i(k} B_{j)^{-h}} & =K_{h i j k}-C_{h i j k}+G_{i(j} E_{k)^{-} h}+G_{h(k} E_{j)^{-i}}, \\
B_{i(j: k)^{-}} & =-C_{\beta i j k} Z^{\beta}+G_{i(k} F_{j)^{-}}, .
\end{aligned}
$$

where the conformal vector $F_{j}$, of class $C^{r-3}$, is defined by the equations

$$
F_{j}=(1 /(n-1)) G^{\left(\sigma_{2}\right)} B_{j g_{1}: g_{2}} \text {. }
$$

These equations are simply equations (22.2), (22.13) and (22.20) respectively after simplification by means of (11.24), (20.10), (20.11), (20.18) and (21.13). Equations (23.23) and (23.24) are the conformal Gauss equations and conformal Codazzi equations respectively for the hypersurface $V_{m-1}$. The vector $F_{j}$ defined by (23.25) plays an important role in the conformal geometry of $V_{m-1}$. It is called the deviation vector.

The remaining conditions are $[2,1]$ and $[2,2]$. The first of these is identical with the conformal Codazzi equations according to the italicized statement of this section. We substitute the values of $U^{\left(h_{2}\right)}{ }_{\left(i_{3}\right)}$ and $U^{h_{1}}{ }_{\left(i_{3}\right)}$ given by (20.18) in (23.6) (condition [2, 2]). To simplify the resulting equations, we note that, according to (11.24) and (20.10), $D_{\alpha \beta j k} G^{\alpha}{ }_{\left(l_{2}\right)} G^{\beta}{ }_{\left(i_{2}\right)}$ $=C_{\alpha \beta j k} Z^{\alpha} Z^{\beta} B_{\left(l_{2}\right)} B_{\left(i_{2}\right)}=0$ and that, according to $\$ 17, B_{\left(i_{2}\right):(j k)^{-}}=B_{g i_{2}} K^{o_{i_{1} j k}}$ $+B_{i_{1 g}} K^{o_{i_{2} j k}}$. If use is made of these equations as well as of (8.3), (20.11) and (21.13), it is found that condition $[2,2]$ is true identically. Therefore conditions $[0,2],[1,1]$ and $[1,2]$ constitute the complete set of integrability conditions of the conformal Frenet equations for a hypersurface. All of the above conditions are valid in any hypersurface of class $C^{r}$ with $r \geqq 4$.

24. The third derivatives of the relative conformal curvature. We assume that (14.8) holds. Then $E_{i j}$ is of class $C^{1}$. The integrability conditions of the 
first set of equations of (20.9) are $\psi_{(i: j)^{-}}=0$. We denote these equations by the symbol $[\psi]$. In view of the second set of (20.9), these integrability conditions are equivalent to

$$
E_{(i j)^{-}}=0 \text {. }
$$

According to $\$ 17$, the integrability conditions of the second set of (20.9) may be written as

$$
\psi_{i:(j k)^{-}}=\psi_{h} K^{h}{ }_{i j k} .
$$

We denote these equations by the symbol $\left[\psi_{i}\right]$. Also, from (20.9),

$$
\begin{aligned}
\psi_{i: j k}= & \psi_{: i k} \psi_{: j}+\psi_{: i} \psi_{: j k}+E_{i j: k}-\mu_{\alpha: k} G^{\alpha}{ }_{i j}-\mu_{\alpha} G^{\alpha}{ }_{i j: k} \\
& +(1 /(m-2))\left[\rho_{\alpha \gamma: \gamma} G^{\alpha}{ }_{i} G^{\beta}{ }_{j} G^{\gamma}{ }_{k}+\rho_{\alpha \beta} G^{\alpha}{ }_{i k} G^{\beta}{ }_{i}+\rho_{\alpha \beta} G^{\alpha}{ }_{i} G^{\beta}{ }_{j k}\right] \\
& -\left(G_{i j} / 2\right)\left[2 e^{2 \psi} \mu^{2} \psi_{: k}+2 e^{2 \psi} \mu \mu_{: k}+2 G^{h} \psi_{: k} \psi: l k\right. \\
& \left.+\left(2 e^{2 \psi} \psi_{: k k}+e^{2 \psi} \rho_{: k}\right) /(m-1)(m-2)\right] .
\end{aligned}
$$

Now equations corresponding to (24.2) and (24.3) are true in any $\bar{V}_{n}$ in $\bar{V}_{m}$. Consequently the replacement principle stated in Theorem 9.2 may be applied if the resulting equations are conformally invariant. This will be seen to be the case.

The value of $\psi_{i: j k}$ given by (24.3) is substituted in (24.2) and the replacement principle is applied. If account is taken of (7.7), (9.7), (9.8), (9.10), (11.11), (12.5), (15.6) and (21.1) with $w=1, v=2$, we readily obtain

$$
\begin{aligned}
E_{i(j: k)^{-}=} & \frac{1}{(n-1)} A_{\alpha \beta} G^{\alpha}{ }_{i(j} G^{\beta}{ }_{k)^{-} h: l} G^{h l}+\frac{1}{(n-1)} C_{\alpha\left(j, G_{k)^{-i}}^{\alpha}\right.} \\
& -\frac{1}{(m-2)}{ }^{0} \rho_{\alpha \beta \gamma} G^{\alpha} G_{i}^{\beta}{ }_{j} G^{\gamma}{ }_{k} .
\end{aligned}
$$

These are the integrability conditions of the second set of equations (20.9). According to the conformal Frenet equations and (21.1),

$$
A_{\alpha \beta} G^{\alpha}{ }_{i j} G_{k h:}^{\beta} G^{h l}=G^{\left(\sigma_{2}\right)} U_{k\left(g_{2}\right)}^{\left(h_{2}\right)} B_{\left(h_{2}\right) \mid i j} .
$$

Also, it follows from $\S 12$ that if $m>3$, the conformal value of ${ }^{0} \rho_{\alpha \beta \gamma} G^{\alpha}{ }_{i} G^{\beta}{ }_{j} G{ }^{\gamma}{ }_{k}$ is given by (12.11) and (12.18). If $m=3, n$ must be 2 . It follows from $\$ \$ 11,12$ that $C^{\alpha}{ }_{\beta \gamma \delta}$ is a zero tensor and that $\rho_{\alpha \beta \gamma}$ is a conformal tensor so that, in this case, ${ }^{0} \rho_{\alpha \beta \gamma}=\rho_{\alpha \beta \gamma}$. Also, if $V_{n}$ is a hypersurface (as is true in the present case $m=3)$, it follows from (20.11), (20.18), (21.13) and (23.25) that

$$
(1 /(n-1)) G^{\left(o_{2}\right)} U^{\left(h_{2}\right)}{ }_{k\left(o_{2}\right)} B_{\left(h_{2}\right) \mid i j}=B_{i j} F_{k} .
$$

If use is made of (24.5), (24.6) and these statements, the integrability conditions (24.4) may be written as 
$(24.7)$

$$
E_{i(j: k)^{-}}=\frac{1}{(n-1)}\left[G^{\left(o_{2}\right)} U^{\left(h_{2}\right)_{k^{-}\left(g_{2}\right)}} B_{\left(h_{2}\right) \mid i \zeta^{-}}+C_{\alpha(j} G_{\left.k)^{-i}\right]}^{\alpha}\right.
$$

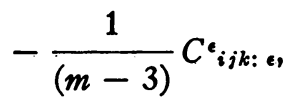

$$
E_{i(j: k)^{-}}=B_{i(j} F_{k)^{-}}-\rho_{\alpha \beta \gamma} G^{\alpha}{ }_{i} G^{\beta}{ }_{j} G^{\gamma}{ }_{k},
$$$$
m=3 \text {. }
$$

Either (24.7) or (24.8), depending upon the value of $m$, are conditions $\left[\psi_{i}\right]$. Also, according to the above remarks, if $V_{n}$ is a hypersurface, (24.7) becomes

$$
\begin{aligned}
E_{i(j: k)^{-}}=B_{i(j} F_{k)^{-}} & +\frac{1}{(n-1)} C_{\alpha\left(j, G_{k)^{-i}}\right.} \\
& -\frac{1}{(m-3)} C_{i j k: \iota,}^{\epsilon_{i j},} \quad m=n+1, m>3 .
\end{aligned}
$$

We now prove the following theorem.

THEOREM 24.1. If $n>3$, the integrability conditions (24.7) are a consequence of the conformal Gauss-Codazzi equations.

Conformal differentiation of the conformal Gauss equations (22.13) leads to the equations

$$
C_{h i j k: l}=K_{h i j k: l}+B_{i(j \mid k)^{-} h: l}+G_{i(j} E_{k)^{-h: l} l}+G_{h(k} E_{j)^{-i: l}} .
$$

To these equations, we add the other two sets obtained by adding cyclic permutations on the indices $j, k, l$ and simplify the result by using (14.9). Now we multiply these last equations by $G^{h l}$ and sum for $h, l$ making use of (21.5). If $m>3$, the left member of these final equations may be replaced by the right member of (12.19). The resulting equations are

$$
\begin{aligned}
& \left.\frac{(n-3)}{(m-3)} C^{\alpha_{i j k: \alpha}}+\frac{1}{(m-3)} G_{i j} C^{\alpha} h l k^{-}: \alpha G^{h l}+C_{\beta(h} G^{\beta}{ }_{j}\right)^{-i} \\
& +C_{\alpha \beta \gamma \delta} G^{h l}\left[G^{\alpha}{ }_{h j}-G^{\beta}{ }_{i} G^{\gamma}{ }_{k}-G^{\delta}{ }_{l}+G^{\alpha}{ }_{h} G^{\beta}{ }_{i j} G^{\gamma}{ }_{j} G^{\delta}{ }_{k}\right] \\
& \left.=\left[B_{i(j \mid k)^{-h: l}}+B_{i l \mid h(j: k)^{-}}\right] G^{h l}+(n-3) E_{i(k: j)^{-}}+G_{i(j} E^{h}{ }_{k}\right)^{-: h} \\
& +E_{:\left(j, G_{k)}-i\right.}
\end{aligned}
$$

where we have written

$$
E^{h}{ }_{k}=G^{h l} E_{l k} .
$$

By means of (11.19) and the conformal Codazzi equations (22.20), the terms involving $C_{\alpha \beta \gamma \delta}$ in (24.10) may be eliminated. Also, the various terms such as $B_{i j \mid h k: l}$ in (24.10) may be eliminated by taking account of (21.10). The resulting equations are 


$$
\begin{aligned}
& \frac{1}{(m-3)}\left[(n-3) C^{\alpha_{i j k: \alpha}}+G_{i j}-C^{\alpha}{ }_{h l k^{-}: \alpha} G^{h l}\right] \\
& \quad+\frac{1}{(n-1)}\left[(n-3) C_{\beta(k} G^{\beta}\right)^{-i}+C_{\beta l} G_{h(j}^{\beta} G_{k)^{-} i} G^{h l} \\
& \quad+(n-3) G^{\left(o_{2}\right)} U^{\left(h_{2}\right)}{ }_{j-\left(o_{2}\right)} B_{\left(h_{2}\right) \mid i h^{-}} \\
& \left.\quad+G^{\left(o_{2}\right)} G^{\left(l_{2}\right)} U^{\left(h_{2}\right)}{ }_{l_{1\left(g_{2}\right)}} B_{\left(h_{2}\right) \mid l_{2}(k} G_{j)^{-i}}\right]=(n-3) E_{i(k: j)^{-}} \\
& \quad+G_{i(j} E_{k)^{-}: h}^{h}+E_{:(j} G_{k)^{-} i} .
\end{aligned}
$$

We multiply these equations by $G^{i k}$ and sum for $i, k$ and obtain

$$
\begin{aligned}
2(n-2)[ & E_{: j}-E^{h}{ }_{j: h}+\frac{1}{(m-3)} C_{h l j: \alpha}^{\alpha} G^{h l} \\
& \left.\quad+\frac{1}{(n-1)}\left(G^{(o 2)} G^{\left(l_{2}\right)} U^{\left(h_{2}\right)}{ }_{l_{1}\left(o_{2}\right)} B_{\left(h_{2}\right) \mid l_{2} j}-C_{\beta l} G_{h j}^{\beta} G^{h l}\right)\right]=0 .
\end{aligned}
$$

If $n=2$, these equations are trivial. However, if $n>2$, and the results of (24.13) are substituted in (24.12), equations (24.12) become

$$
(n-3) E_{i(j: k)^{-}}=\frac{(n-3)}{(n-1)}\left[G^{\left(\rho_{2}\right)} U^{\left(h_{2}\right)}{ }_{k^{-}\left(o_{2}\right)} B_{\left(h_{2}\right) \mid i j^{-}}+C_{\alpha(j} G^{\alpha}{ }_{k)^{-i}}\right]
$$

$$
-\frac{(n-3)}{(m-3)} C_{i j k: \epsilon \cdot}
$$

If $n=3$, these equations are true trivially. However, if $n>3,(24.14)$ coincides with (24.7). This completes the proof. Incidentally, we note that if $n=3$, equations (24.13) which may be obtained from (24.7) by multiplying with $G^{\text {ik }}$ and summing on $i, k$ are a consequence of the conformal Gauss-Codazzi equations.

Another set of partial differential equations satisfied by the deviation tensor $E_{i j}$ is now derived from the conformal Gauss equations if $n>2$. These equations are not related to the integrability conditions (24.7)." As has been noted in $\$ 22,(22.9)$ and (22.10) are consequences of the conformal Gauss equations. From these two sets of equations, we readily obtain

(24.15) $(n-2) E_{i j}=B_{i j}+C_{i j}-K_{i j}-\frac{1}{2(n-1)}(B+C-K) G_{i j}$

after account is taken of (22.17) and (22.18). Conformal differentiation of (24.15) leads to the differential equations

$$
(n-2) E_{i(j: k)^{-}}=\mathcal{B}_{i j k}+C_{i j k}-K_{i j k}
$$

where $K_{i j k}$ is defined by (14.14) and $B_{i j k}$ and $C_{i j k}$ are defined by the analogous equations 


$$
\begin{aligned}
& \mathbb{B}_{i j k}=\mathcal{B}_{i(j: k)^{-}}+\frac{1}{2(n-1)} \mathcal{B}_{:(j} G_{k)^{-i}}=\mathbb{B}_{i(j: k)^{-},} \\
& C_{i j k}=C_{i(j: k)^{-}}+\frac{1}{2(n-1)} C_{:\left(j, G_{k}\right)^{-i}} .
\end{aligned}
$$

25. The second derivatives of the mean curvature. Discussion for a hypersurface. In obtaining the integrability conditions of the last set of equations (20.9), we divide our work into two sections. In this section, we consider the simplest case only, that is, the case where $V_{n}$ is a hypersurface of $V_{m}$ so that $m=n+1$. In this instance the calculations are considerably shorter than for a general subspace of $V_{m}$. The general case is considered in the following section. For the work of this section, we assume that (12.23) holds.

For a hypersurface, the last set of equations (20.9) may be replaced by (20.23). If account is taken of (23.25), equations (20.23) may be written as

$$
\mu_{: i}=e^{-\psi}\left[F_{i}+B_{j h} \psi: i G^{h l}-\frac{1}{(n-1)} \rho_{\alpha \beta} Z^{\alpha} G^{\beta}{ }_{i}\right] .
$$

The integrability conditions of these equations are

$$
\mu:(j k)^{-}=0 .
$$

The replacement principle may be applied to equations (25.1) and (25.2) for reasons like those stated in the beginning of the preceding section. This means that (25.2) is equivalent to

$$
{ }^{0} \mu_{:(j k)^{-}}=0 .
$$

Conformal differentiation of (25.1) leads to equations in which we make replacements using (9.7), (13.15), (20.13) and (20.14). In this way, we obtain the equations

$$
\begin{aligned}
{ }^{0} \mu_{: j k}= & F_{j: k}+B_{j h} E_{l k} G^{h l}+\frac{1}{(m-2)}{ }^{0} \rho_{\alpha \beta} G^{\alpha}{ }_{l} G^{\beta}{ }_{k} B_{j h} G^{h l} \\
& -\frac{{ }^{0} \rho}{2(m-1)(m-2)} B_{j k}-\frac{1}{(n-1)}{ }^{0} \rho_{\alpha \beta: \gamma} Z^{\alpha} G_{j}^{\beta} G^{\gamma_{k}} \\
& +\frac{1}{(n-1)}{ }^{0} \rho_{\alpha \beta}\left[B_{k h} G^{h l} G^{\alpha}{ }_{l} G^{\beta}{ }_{j}-Z^{\alpha} Z^{\beta} B_{j k}\right] .
\end{aligned}
$$

Now $m=n+1$ and $\rho_{\alpha \beta}, B_{j k}$ are symmetric tensors and ${ }^{0} \rho_{\alpha \beta ; \gamma}={ }^{0} \rho_{\alpha \beta, \gamma}$. Hence substitution of (25.4) in (25.3) leads to the equations

$$
F_{(j: k)^{-}}=B_{h(k} E^{h}{ }_{j)^{-}}+\frac{1}{(n-1)}{ }^{0} \rho_{\alpha \beta \gamma} Z^{\alpha} G^{\beta}{ }_{j} G^{\gamma}{ }_{k}
$$


where $\rho_{\alpha \beta \gamma}$ is defined by (12.5) and account has been taken of (11.21). These equations are the integrability conditions of (25.1). As in the case of (24.4), the integrability conditions (25.5) may be written as

$$
\begin{array}{ll}
\left.F_{(j: k)^{-}}=B_{h(k} E^{h_{j}}\right)^{-}+\rho_{\alpha \beta \gamma} Z^{\alpha} G^{\beta}{ }_{j} G_{k,}, & n=2, \\
\left.F_{(j: k)^{-}}=B_{h(k} E^{h_{j}}\right)^{-}+\frac{1}{(n-2)} C^{{ }_{\alpha j k:}{ }_{\alpha j} Z^{\alpha},} & n>2,
\end{array}
$$

where $C^{\epsilon_{\alpha j k: e}}$ is defined by (12.21). We denote the integrability conditions (25.6) or (25.7) by the symbol $[\mu]$.

We now prove the following theorem.

THEOREM 25.1. If $n>2$, the integrability conditions (25.7) are a consequence of the conformal Gauss-Codazzi equations.

We first derive some equations which are needed in the proof of this theorem. Conformal differentiation of the conformal Codazzi equations (23.24) leads to

$$
B_{j(i: k)^{-} l}=-\left(C_{\alpha j i k} Z^{\alpha}\right)_{: l}+G_{j(k} F_{i)^{-}: l} .
$$

From the Ricci identities of $\$ 17$,

$$
B_{i j:(l k)^{-}}=B_{h j} K^{h}{ }_{i l k}+B_{i h} K^{h}{ }_{j l k} .
$$

From (25.8) and (25.9), we obtain

$$
\text { (25.10) } B_{j i: l k}=B_{j k: i l}-\left(C_{\alpha j i k} Z^{\alpha}\right)_{: l}+G_{j(k} F_{i)^{-}: l}+B_{h j} K^{h_{i l k}}+B_{i h} K^{h}{ }_{j l k} .
$$

We multiply (25.10) by $G^{i l}$ and sum for the indices $i, l$. In the resulting equations, we apply the alternation operation to the indices $j, k$ and simplify, using (11.14), (14.4) and (23.25). In this way, we find that

$$
(n-2) F_{(j: k)^{-}}=\left[C_{\alpha \beta \gamma \delta} Z^{\alpha} G^{\beta} G^{\gamma}{ }_{k} G^{\delta_{j}}\right]_{:} G^{i l}+G^{i l} B_{h i} K^{h}{ }_{l j k}+B_{h(j} K^{h}{ }_{k)^{-}} .
$$

If use is made of (7.10), (8.13), (20.13) and (20.14), we find that

$$
\begin{aligned}
{\left[C_{\alpha \beta \gamma \delta} Z^{\alpha} G^{\beta}{ }_{i} G^{\gamma}{ }_{k} G^{\delta}{ }_{j}\right]_{:} G^{i l}=} & C_{\alpha \beta \gamma \delta:} Z^{\alpha} G^{\beta \iota} G^{\gamma}{ }_{k} G^{\delta}{ }_{j} \\
& +C_{\alpha \beta \gamma \delta} G^{i l} Z^{\alpha} G^{\beta_{i}}\left[B_{k l} Z^{\gamma} G^{\delta}{ }_{j}+B_{j l} G^{\gamma}{ }_{k} Z^{\delta}\right]
\end{aligned}
$$

since $C_{\alpha \beta \gamma \delta} Z^{\alpha} Z^{\beta}=0, C_{\alpha \beta \gamma \delta} G^{i l} G^{o h} B_{l o} G^{\alpha}{ }_{h} G^{\beta}{ }_{i}=0$. Now $G^{\beta \epsilon}=A^{\beta \epsilon}-Z^{\beta} Z^{\epsilon}$, so that $C_{\alpha \beta \gamma \delta: \epsilon} Z^{\alpha} G^{\beta \epsilon} G^{\gamma}{ }_{k} G^{\delta}{ }_{j}=C^{\epsilon_{\alpha \beta \gamma: \varepsilon}} Z^{\alpha} G^{\beta}{ }_{j} G^{\gamma}{ }_{k}$. If this is substituted in (25.12) and the result is then substituted in (25.11), we obtain

$$
\begin{aligned}
& (n-2) F_{(j: k)^{-}}=C^{\epsilon_{\alpha j k:}} Z^{\alpha}+C_{\alpha \beta \gamma \delta} Z^{\alpha} G^{i l} G^{\beta}{ }_{i} Z^{\gamma} G^{\delta}{ }_{(j} B_{k)^{-}} \\
& +G^{i l} B_{h i} K^{h}{ }_{l j k}+B_{h(j} K^{h}{ }_{k)} \text {. }
\end{aligned}
$$

These equations are consequences of the conformal Codazzi equations.

Also, from the conformal Gauss equations (23.23), we may obtain equa- 
tions for the tensors $G^{i l} B_{h i} K^{h}{ }_{l j k}, B_{j h} K^{h_{k}}$ and $B_{h k} K^{h}{ }_{j}$ which occur in (25.13). From these equations we find that

$$
G^{i l} B_{h i} K^{h}{ }_{l j k}+B_{h(j} K^{h}{ }_{k)^{-}}=C_{\alpha \delta} G^{\alpha}{ }_{i} G^{\delta}{ }_{(k} B_{j)^{-}} G^{i l}+(n-2) B_{h(k} E^{h}{ }_{j)^{-}}
$$

after account is taken of (11.16) and of the equations $C_{\alpha \beta \gamma \delta} G^{\alpha}{ }_{i} G^{\beta}{ }_{h} G^{\gamma}{ }_{j} G^{{ }^{b}}{ }_{k} G^{i l} G^{h o} B_{g l}$ $=0$ which are a consequence of (11.14) and (23.22). Now, if use is made of (11.22), we find that (25.14) becomes

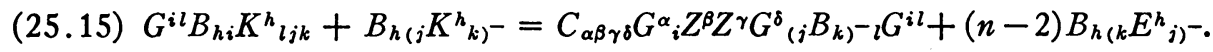

These equations are consequences of the conformal Gauss equations.

If (25.13) is simplified by means of (25.15) and use is made of (11.14), we find that

$$
(n-2) F_{(j: k)^{-}}=(n-2) B_{h(k} E^{h}{ }_{j)^{-}}+C_{\alpha j k: \epsilon^{\epsilon^{\alpha}}} Z^{\alpha}
$$

is a consequence of the conformal Gauss-Codazzi equations. If $n>2$, these equations are equivalent to (25.7). This completes the proof of the theorem.

26. The second derivatives of the mean curvature normal. Discussion for the general case. We return to a consideration of the last set of equations (20.9) in the general case. We assume that (14.8) holds. According to (17.1), the integrability conditions of these equations are $\mu^{\alpha}:(j k)^{-}=-\mu^{\beta} R^{\alpha}{ }_{\beta j k}$. As in the two previous sections, the replacement principle may be applied to these equations. After replacements and utilization of (9.7), the integrability conditions become

$$
{ }^{0} \mu^{\alpha}:(j k)^{-}=0 .
$$

In the calculations that follow, only the main links in the chain of the argument are given explicitly and the intervening links are only stated in outline form. This is done in order to avoid the appearance of several lengthy tensor equations which do not differ essentially from those which actually occur in the following calculations. An expression for ${ }^{0} \mu^{\alpha}: j k$ may be obtained by conformal differentiation of the last set of equations (20.9) and employment of the replacement principle. If this expression is substituted in (26.1) and the result simplified by means of (8.15), (9.7), (9.10), (12.5), (13.15), (15.6) and (20.9), we obtain the equivalent integrability conditions

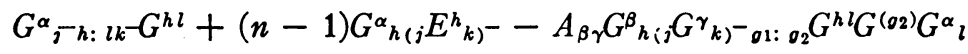

$$
\begin{aligned}
& +C_{\beta \gamma} G^{\beta}{ }_{h(j} G^{\gamma}{ }_{k)}-G^{h l} G^{\alpha}{ }_{l}+G^{\alpha}{ }_{h(k} K^{h}{ }_{j)^{-}}+G^{\alpha}{ }_{h} K^{h}{ }_{(j: k)^{-}} \\
& \left.+(n-2) G^{\alpha}{ }_{h(k} E^{h}{ }_{j}\right)^{-}+(n-2) G^{\alpha}{ }_{h} E^{h}{ }_{(j: k)^{-}}+E_{:(k} G^{\alpha}{ }_{j)^{-}} \\
& +C^{\alpha}{ }_{\beta \gamma-\delta: ~}{ }^{-}-G^{\beta \delta} G^{\gamma}{ }_{j} G^{\epsilon_{k}}-\frac{(n-1)}{(m-2)}{ }^{0} \rho_{\beta \gamma \delta} G^{\gamma_{j}} G^{\delta}{ }_{k} H^{\alpha \beta} \\
& +C^{\alpha}{ }_{\beta \gamma \delta} G^{h l} G^{\gamma}{ }_{j}\left[G^{\beta}{ }_{h k}-G^{\delta}{ }_{l}+G^{\beta}{ }_{h} G^{\delta}{ }_{l k}-\right]=0 .
\end{aligned}
$$

The terms involving $K^{h}{ }_{j}$ and $K^{h}{ }_{j: k}$ are replaced by means of (12.20), 
(12.22) and (22.10). The terms involving( $\left.{ }^{41}\right) C^{\alpha}{ }_{\beta \gamma 8: \epsilon}$ are replaced by the use of (12.12) and the value of ${ }^{0} \rho_{\beta \gamma \delta}$ is given by (12.11). After these various substitutions and further simplifications by means of the symmetry properties of the Weyl space tensor, (26.2) becomes

$$
\begin{aligned}
& G^{\alpha}{ }_{j}{ }^{h}: l k-G^{h l}+(n-1) G^{\alpha}{ }_{h k^{-}}\left[\mathscr{B}^{h}{ }_{j}+C^{h}{ }^{-}-(n-1) E^{h}{ }_{j}\right] \\
& +G^{\alpha}{ }_{h}\left[\mathrm{~B}^{h}{ }_{(j: k)^{-}}-A_{\beta \gamma} G^{\beta}{ }_{l(j,} G^{\gamma}{ }_{k)^{-}{ }^{\prime} 1: \rho_{2}} G^{h l} G^{\left(\rho_{2}^{\prime}\right)}\right] \\
& +H^{\alpha \epsilon}\left[\left(C_{\epsilon \beta \gamma \delta}+C_{\epsilon \delta \gamma \beta}\right) G_{h(k}^{\beta} G^{\gamma}{ }_{j)}-G^{\delta}{ }_{l} G^{h l}+C_{\epsilon \beta j k: \gamma} G^{\beta \gamma}\right.
\end{aligned}
$$$$
\left.+\frac{1}{(m-3)} C_{\epsilon k j: \zeta}\right]=0 \text {. }
$$

Now, from (20.7), we find that

$$
G^{\alpha}{ }_{j h: l k}=G^{\alpha}{ }_{j h l k}+\left[U^{\left(h_{3}\right)}{ }_{j h l k}+U^{\left(h_{2}\right)_{j h}} G^{h_{3}}{ }_{k}\right] G^{\alpha}{ }_{\left(h_{3}\right)}
$$

and that

$$
\begin{aligned}
& +\left[U^{\left(h_{2}\right)}{ }_{j h l k}+U^{\left(h_{2}\right)}{ }_{j h l: k}+U^{\left(o_{2}\right)}{ }_{j h l} U^{\left(h_{2}\right)}{ }_{\left(o_{2}\right) k}+U^{h_{1}{ }_{j h l} G^{h_{2}}}\right] G^{\alpha}{ }_{\left(h_{2}\right)} \\
& +\left[U^{\left(o_{2}\right)}{ }_{j h l} U^{h_{1}}{ }_{\left(o_{2}\right) k}+U^{h_{1}}{ }_{j h l: k}\right] G^{\alpha}{ }_{h_{1}}
\end{aligned}
$$

Also, as a consequence of (12.20),

$$
\begin{aligned}
& H^{\alpha \epsilon}\left[\left(C_{\epsilon \beta \gamma \delta}+C_{\epsilon \delta \gamma \beta}\right) G_{h(k}^{\beta} G_{j}^{\gamma}\right)-G^{\delta}{ }_{l} G^{h l}\left.+C_{\epsilon \beta j k: \gamma} G^{\beta \gamma}+\frac{1}{(m-3)} C_{\epsilon k j: \zeta}^{\zeta}\right] \\
&=H^{\alpha \epsilon}\left[C_{\epsilon(k: j)}-+\frac{(n-1)}{(m-3)} C_{\epsilon k j: \zeta}\right] .
\end{aligned}
$$

We substitute these values in (26.3) and multiply successively by $A_{\alpha \beta} G^{\beta}{ }_{\left(i_{v}\right)}$ $(v=1,2, \cdots, M, \infty)$ and simplify using (18.13), (21.1) and (21.2). In this way we obtain the following equations which are equivalent $\left({ }^{42}\right)$ to the integrability conditions (26.1) if $m$ exceeds 3 :

$$
\begin{aligned}
& {\left[U^{\left(h_{2}\right)}{ }_{j} h l k^{-}+U^{\left(h_{2}\right)},-h l: k^{-}+U^{\left(o_{2}\right)}{ }_{j}-h_{l} U^{\left(h_{2}\right)}{ }_{\left(o_{2}\right) k^{-}}\right.}
\end{aligned}
$$

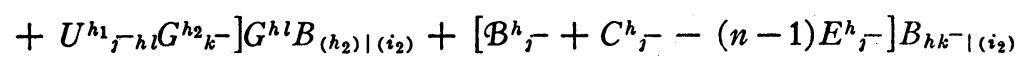

$$
\begin{aligned}
& =\left[C_{\epsilon(j: k)}-+\frac{(n-1)}{(m-3)} C_{\epsilon j k: \zeta}^{\xi}\right] G_{\left(i_{2}\right)}^{\epsilon}, \\
& {\left[U^{\left(h_{3}\right)} \tau_{h} l k^{-}+U^{\left(h_{2}\right)}{ }_{j} t G^{h_{3}}{ }_{k^{-}}\right] G^{h l} B_{\left(h_{3}\right) \mid\left(i_{3}\right)}} \\
& =\left[C_{\epsilon(j: k)^{-}}+\frac{(n-1)}{(m-3)} C_{\epsilon j k: \zeta}\right] G^{\epsilon}{ }_{\left(i_{3}\right)},
\end{aligned}
$$

(41) The assumption $m>3$ is implicit in these substitutions. In the remaining case, $m=3$, it follows that $n=2$. This case is discussed in $\$ 25$.

${ }^{(42}$ ) This equivalence has been established by the use of the following equations of chapter $\mathrm{V}:[1,1],\left[\begin{array}{ll}B & 1\end{array}\right],\left[\begin{array}{ll}B & 2\end{array}\right],\left[\begin{array}{ll}D & 1\end{array}\right]$ with $w=1$. 


$$
\begin{gathered}
B_{j^{-} h l k^{-} \mid\left(i_{4}\right)} G^{h l}=\left[C_{\epsilon(j: k)^{-}}+\frac{(n-1)}{(m-3)} C_{\epsilon j k: \zeta}\right] G_{\left(i_{4}\right)}, \\
{\left[C_{\epsilon(j: k)^{-}}+\frac{(n-1)}{(m-3)} C_{\epsilon j k: \zeta}\right] G_{\left(i_{u}\right)}=0, \quad u=5,6, \cdots, M, \infty .}
\end{gathered}
$$

We denote the integrability conditions (26.7), (26.8), (26.9) and (26.10) by the symbols $\left[\mu^{\alpha}, 2\right],\left[\mu^{\alpha}, 3\right],\left[\mu^{\alpha}, 4\right]$ and $\left[\mu^{\alpha}, u\right], u>4$, respectively. In writing these equations, we have omitted the first set $(v=1)$ obtained by multiplying (26.3) by $A_{\alpha \beta} G^{\beta}{ }_{i}$. For this first set of equations becomes an identity after application is made of (7.11) [equivalent to (21.5)] and (21.9) with $w=1$.

For a hypersurface, $m=n+1$ and $M=2$. In this case, (26.8) to (26.10) become vacuous so that (26.7) are the only integrability conditions. These equations (26.7) are equivalent to the integrability conditions (25.7) previously obtained for a hypersurface. Consequently the integrability conditions (25.7) for a hypersurface are $\left[\mu^{\alpha}, 2\right]$. The proof of this statement follows. The expression which multiplies $B_{\left(h_{2}\right) \mid\left(i_{2}\right)}$ in (26.7) is equal to $A_{\alpha \beta} G^{\alpha}{ }_{j^{-h l k}}-G^{h l} G^{\beta}{ }_{\left(i_{2}\right)}$. Now for a hypersurface, according to (11.21), (20.10), (20.13) and (20.14),

$$
A_{\alpha \beta} G^{\alpha}{ }_{j} h l k-G^{\beta}{ }_{\left(i_{2}\right)} G^{h l}=B_{j-h: l k}-G^{h l} B_{\left(i_{2}\right)} .
$$

Also, from (11.23), (20.10) and (20.14),

$$
C_{\epsilon j: k} G^{\epsilon}{\left(i_{2}\right)}=\left[\left(C_{\epsilon j} Z^{\epsilon}\right)_{: k}-C_{\epsilon j} Z^{\epsilon}: k\right] B_{\left(i_{2}\right)}=C_{h j} B^{h} \cdot B_{\left(i_{2}\right)} .
$$

Also, from (20.11) and (22.18),

$$
\mathcal{B}^{h}{ }_{(j} B_{k)^{-} h \mid\left(i_{2}\right)}=B^{h}{ }_{g_{1}} B_{o_{2}(j} B_{k)^{-} h} B_{\left(i_{2}\right)} G^{\left(g_{2}\right)}=0 .
$$

If the results stated in (26.11), (26.12) and (26.13) are substituted in (26.7) and account is taken of (23.25), we obtain (25.7).

We now return to a consideration of the general case and prove the following theorem which is a generalization of Theorem 25.1:

THEOREM 26.1. If $n>2$, the integrability conditions (26.7), (26.8), (26.9) and $(26.10)$ are a consequence $\left({ }^{43}\right)$ of the equations $[1, v]$ and $[2, v], v=1,2, \cdots$, $M, \infty$.

The first stage of the proof consists in obtaining new tensor equations which may be solved for the expression $G^{\alpha}{ }_{j}{ }^{h}: l k^{-}-G^{h l}$ occurring in (26.3). Conditions $[1, v]$ are equivalent to the equations $G^{\alpha}{ }_{j h: k}=G^{\alpha}{ }_{j k: h}+G^{\alpha}{ }_{0} K^{{ }_{j}}{ }_{j h k}-R^{\alpha}{ }_{j h k}$.

$\left.{ }^{43}\right)$ The proofs of the theorems of $\$ \$ 24-26$ also depend upon the definitions and identities of chapter III, the extended conformal Frenet equations and the $A, D$ and $B$ conditions. However, if $V_{m}$ is an $\bar{R}_{m}$, the extended conformal Frenet equations and $B$-conditions (which involve space indices) may be omitted from this list. Some of the above equations are also used in the derivation of the various integrability conditions which appear in $\$ \$ 24-26$. 
Conformal differentiation of these equations with respect to $x^{l}$ leads to

$$
G^{\alpha}{ }_{j h: k l}=G^{\alpha}{ }_{j k: h l}+G^{\alpha}{ }_{o l} K^{o}{ }_{j h k}+G^{\alpha}{ }_{0} K^{o}{ }_{j h k: l}-R^{\alpha}{ }_{j h k: l} .
$$

Now conditions $[2, v]$ are equivalent to

$$
G^{\alpha}{ }_{j h: l k}=G^{\alpha}{ }_{j h: k l}+G^{\alpha}{ }_{j h} K^{o}{ }_{j l k}+G^{\alpha}{ }_{j o} K^{j}{ }_{h l k}-R^{\alpha}{ }_{\beta l k} G^{\beta}{ }_{j h} .
$$

We substitute the value for $G^{\alpha}{ }_{j h: k l}$ given by (26.14) in (26.15). Then we multiply the equations by $G^{h l}$ and perform the alternation operation on the indices $j, k$. In this manner, we obtain

$$
\begin{aligned}
G^{\alpha}{ }_{j-h: l k}-G^{h l}= & 2 G^{\alpha}{ }_{0 l} K^{o}{ }_{j-h k}-G^{h l}+G^{\alpha}{ }_{h(j} K^{h}{ }_{k)^{-}}+G^{\alpha}{ }_{0} K^{o}{ }_{j}-h k^{-}: l G^{h l} \\
& -R^{\alpha}{ }_{j-h k^{-}: l} G^{h l}-R^{\alpha}{ }_{\beta l(k} G^{\beta}{ }_{j)}{ }^{-} G^{h l}
\end{aligned}
$$

after taking account of (14.2).

We replace the conformal Riemann tensor in these equations by (16.9) and (16.10) and then simplify using (11.14) and (14.4). In this way (26.16) becomes

$$
\begin{aligned}
& \frac{(n-2)}{(n-1)} G^{\alpha}{ }_{j-h: l k-} G^{h l}=G^{\alpha}{ }_{h(j} K^{h}{ }_{k)^{-}}+G^{\alpha}{ }_{0} K^{o}{ }_{h j k: l} G^{h l} \\
& +\left[C^{\alpha}{ }_{h k j}+\frac{1}{(n-1)} H^{\alpha \beta} C_{\beta(k} G_{i)^{-h}}\right]:{ }_{l} G^{h l} \\
& +G^{\alpha}{ }_{(k} E^{h}{ }_{j)^{-}: h}+G^{\alpha}{ }_{h} E^{h}{ }_{(k: j)^{-}}+C^{\alpha_{\beta l(j} G^{\beta}{ }_{k)^{-}{ }_{h}} G^{h l}} \\
& +\frac{1}{(n-1)} G^{h l} G^{\beta}{ }_{h k^{-}}\left[C_{\beta}, G^{\alpha}{ }_{l}-C_{\beta l} G^{\alpha}{ }^{-}\right] \\
& +\frac{1}{(n-1)}\left[G^{\alpha}{ }_{h(k} \mathrm{B}^{h}{ }_{i)^{-}}-+G^{\alpha}{ }_{h} \mathrm{~B}^{h}{ }_{(j: k)^{-}}\right] \\
& +\frac{1}{(n-1)} G^{\left(o_{2}\right)} G^{h l} A_{\gamma \beta} G^{\beta}{ }_{h-}-\left[G^{\gamma_{k^{-} \sigma_{1}: o_{2}}} G^{\alpha}{ }_{l}-G^{\gamma_{l 0_{1}: o_{2}}} G^{\alpha_{k}-}\right] \text {. }
\end{aligned}
$$

Now if use is made of (12.12), (12.21) and (11.14), we find that

$$
\begin{aligned}
& {\left[C^{\alpha k k j}+\frac{1}{(n-1)} H^{\alpha \beta} C_{\beta(k} G_{j)^{-} h}\right]: l G^{h l}=C^{\alpha k j: \mathrm{e}} G^{\beta e}}
\end{aligned}
$$

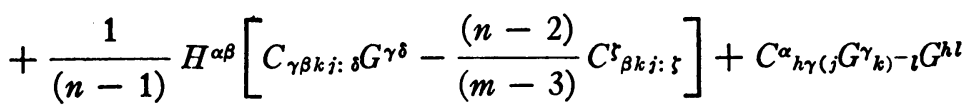

$$
\begin{aligned}
& +\frac{1}{(n-1)} H^{\alpha \beta}\left[C_{h \beta k^{-} \delta}+C_{\delta \beta k^{-} h}\right] G^{\delta_{l}-G^{h l}}+C_{h(j} G^{\alpha}{ }_{k)-}{ }^{-} G^{h l} \\
& +C_{\beta(2} G^{\beta}{ }_{k)}-T_{2} G^{h l} G^{\alpha}{ }_{h}
\end{aligned}
$$


Also, from (22.10), which is a consequence of condition $[1,1]$, we find that (26.19)

$$
G^{\alpha}{ }_{h(j} K^{h}{ }_{k)^{-}}=G^{\alpha}{ }_{h(j} \mathrm{B}^{h}{ }_{k)^{-}}+G^{\alpha{ }_{h(j} C^{h}{ }_{k)^{-}}}+(2-n) G^{\alpha}{ }_{h(j} E^{h}{ }_{k)^{-}} .
$$

From (22.10) and (12.12), we obtain

$$
\begin{aligned}
& G^{\alpha}{ }_{0} K^{o}{ }_{h j k:} G^{h l}=G^{\alpha}{ }_{0} G^{h l}\left[-K^{o}{ }_{h l j: k}-K^{o}{ }_{h k l: j}\right]=G^{\alpha}{ }_{h} K^{h}{ }_{(k: j)}- \\
& =G^{\alpha}{ }_{h} \mathrm{~B}^{h}{ }_{(k: j)^{-}}+\left(C_{\epsilon \beta k^{-} l}+C_{l \beta k^{-}}\right) G^{{ }^{6}{ }_{h}-G^{h l} G^{\alpha \beta}} \\
& +C_{\gamma \beta k j:{ }_{\delta} G^{\gamma \delta} G^{\alpha \beta}}+\frac{(n-3)}{(m-3)} C_{\beta j k: 5} G^{\alpha \beta} \\
& +\frac{1}{(m-3)} G^{\alpha}{ }_{j}-C^{\zeta}{ }_{\gamma \delta k^{-}: 5} G^{\gamma \delta}+C_{\beta(k} G^{\beta}{ }_{j)^{-}} G^{\alpha}{ }_{l} G^{h l} \\
& +E_{:(k} G^{\alpha}{ }_{i)^{-}}+(n-2) G^{\alpha} E^{h}{ }_{(j: k)^{-}} \text {. }
\end{aligned}
$$

If the results given by equations (26.18), (26.19) and (26.20) are substituted in (26.17), and account is taken of the fact that $H^{\alpha \beta}=A^{\alpha \beta}-G^{\alpha \beta}$ and of (11.14), equations (26.17) become

$$
\begin{aligned}
& \frac{(n-2)}{(n-1)} G^{\alpha}{ }_{j}-l: l k-G^{h l}=\frac{n^{2}-5 n+5}{(m-3)(n-1)} C_{\beta j k: 5} G^{\alpha \beta}+\frac{(n-2)}{(n-1)} C_{\gamma \beta j k: \delta} G^{\gamma \delta} H^{\alpha \beta} \\
& +\frac{(n-2)}{(m-3)(n-1)} C^{\zeta \alpha}{ }_{j k: \zeta}+\frac{1}{(m-3)} G^{\alpha}, C^{\zeta}{ }_{\gamma \delta k^{-}: 5} G^{\gamma \delta} \\
& +\frac{1}{(n-1)} G^{h l} C_{\beta \gamma}\left[(n-3) G^{\beta}{ }_{h(j} G^{\left.\gamma_{k}\right)-G^{\alpha}{ }_{l}}\right. \\
& \left.+G^{\beta}{ }_{h(j} G^{\alpha}{ }_{k)}-G^{\gamma}{ }_{l}+(n-2) G^{\beta}{ }_{h} G^{\gamma}{ }_{(k} G^{\alpha}{ }_{j)}{ }^{-l}\right] \\
& +\frac{(n-2)}{(n-1)}\left(C_{\beta \gamma l J^{-}}+C_{\beta l \gamma j^{-}}\right) G^{\gamma} h k-G^{h l} H^{\alpha \beta} \\
& +\frac{(n-2)}{(n-1)}\left[G^{\alpha}{ }_{h(j} \mathrm{B}^{h}{ }_{k)}-+G^{\alpha}{ }_{h} \mathrm{~B}^{h}{ }_{(k: j)}-\right] \\
& +(n-2) G^{\alpha}{ }_{h(k} E^{h}{ }_{j)^{-}}+(n-3) G^{\alpha}{ }^{\alpha} E^{h}{ }_{(j: k)^{-}} \\
& +E_{:(k} G^{\alpha}{ }_{j)^{-}}+G^{\alpha}{ }_{(k} E^{h}{ }_{j)^{-}: h}
\end{aligned}
$$

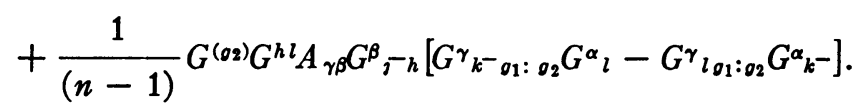

The derivation of equations $(26.21)$ by the use of conditions $[1, v]$ and $[2, v]$. completes the first stage of the proof.

To complete the proof of the theorem, we now show that the integrability conditions (26.3) [equivalent to (26.7) through (26.10)] become identities when use is made of (26.21). If $n>2$, we may solve (26.21) for $G^{\alpha}{ }_{5}{ }^{-h: l k}-G^{h l}$ and substitute the result in (26.3). When the result is simplified, (26.3) be- 
comes, after multiplication by the factor $(n-2)$,

$$
\begin{aligned}
& \left.\frac{(n-1)}{(m-3)}\left[(n-3) C_{\beta j k: 5} G^{\alpha \beta}+G^{\alpha}{ }_{j}-C^{\zeta}{ }_{\gamma \delta k^{-}: 5} G^{\gamma \delta}\right]+(n-3) C_{\beta(k} G^{\beta}{ }_{j}\right)^{-}{ }_{h} G^{\alpha}{ }_{l} G^{h l} \\
& +C_{\beta l} G^{\beta}{ }_{h(j} G^{\alpha}{ }_{k)}-G^{h l}+(n-1)(n-3) G^{\alpha}{ }_{h} E^{h}{ }_{(j: k)^{-}} \\
& \left.+(n-1) E_{:(k} G^{\alpha}{ }_{j)^{-}}+(n-1) G^{\alpha}{ }_{(k} E^{h}{ }^{h}\right)^{-:}: h
\end{aligned}
$$

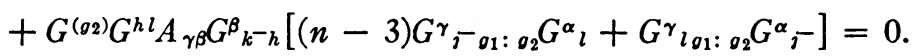

Since all the indices of (26.22) are tangent indices, equations (26.22) are equivalent to the ones obtained from them by multiplication with $A_{\alpha \epsilon} G_{i}$. These new equations are

$$
\begin{aligned}
& \frac{(n-1)}{(m-3)}\left[(n-3) C^{\zeta j k: \zeta}+G_{i,}-C^{\zeta}{ }_{\gamma \delta k^{-}: 5} G^{\gamma \delta}\right]+(n-3) C_{\beta(k} G^{\beta}{ }_{j)^{-i}} \\
& +C_{\beta l} G^{h l} G^{\beta}{ }_{h(j} G_{k)^{-i}}+(n-1)(n-3) E_{i(j: k)^{-}}+(n-1) E_{:(k} G_{j)^{-i}} \\
& +(n-1) G_{i(k} E^{h}{ }_{j)^{-:} h}+(n-3) G^{\left(a_{2}\right)} B_{\left(h_{2}\right) \mid i(k} U^{\left(h_{2}\right)}{ }_{j)^{-}}{ }^{\left(o_{2}\right)} \\
& +G^{\left(g_{2}\right)} G^{h l} U^{\left(h_{2}\right)}{ }_{l\left(g_{2}\right)} B_{\left(h_{2}\right) \mid h(k} G_{j)^{-i}}=0
\end{aligned}
$$

after use is made of (26.5). If we multiply (26.23) by $G^{i k}$ we obtain

$$
2(n-2)\left[\frac{(n-1)}{(m-3)} C_{\gamma \delta j: 5} G^{\gamma \delta}-C_{\beta} l G^{\beta}{ }_{h j} G^{h l}+U^{\left(h_{2}\right)}{ }_{l\left(o_{2}\right)} B_{\left(h_{2}\right) \mid j h} G^{\left(o_{2}\right)} G^{h l}\right.
$$

$$
\left.+(n-1)\left(E_{: j}-E^{h}{ }_{j: h}\right)\right]=0 .
$$

Since $n>2$, we may solve (26.24) for $C^{\zeta}{ }_{\gamma^{\delta} j: 5} G^{\gamma^{\delta}}$ and substitute in (26.23). Then (26.23) becomes

$$
(n-3)\left[\frac{(n-1)}{(m-3)} C_{i j k: \zeta}+C_{\beta(k} G^{\beta}{ }_{i)^{-i}}+(n-1) E_{i(j: k)^{-}}\right.
$$

$$
\left.+G^{\left(\theta_{2}\right)} B_{\left(h_{2}\right) \mid i(k} U^{\left(h_{2}\right)}{ }_{i)^{-}\left(o_{2}\right)}\right]=0 .
$$

If $n=3,(26.25)$ is true identically so that (26.24) is equivalent to (26.23). According to the italicized statement following (24.14), equations (26.24) [which are identical with (24.13)] are a consequence of conditions $[1,1]$ and $[1,2]$. Consequently this is also true for (26.23). If $n>3,(26.25)$ is equivalent to (26.23) since (26.24) may be obtained from (26.25) by summing with $G^{i h}$ and (26.23) follows from (26.24) and (26.25). According to Theorem 24.1, equations (26.25) [which are identical with (24.7)] are a consequence of conditions $[1,1]$ and $[1,2]$. Therefore this is also true for $\left(26.2^{2)}\right.$ The preceding discussion of the two cases $n=3$ and $n>3$ shows that, after $\downarrow$ se of $[1,1]$ and 
[1, 2 ], (26.23) is an identity if $n>2$. Since (26.23) is equivalent to the integrability conditions $(26.3)$ by means of the conditions $[1, v]$ and $[2, v]$, the above remark completes the proof of Theorem 26.1. More precisely, the proof of this theorem shows that, for a fixed $v$ between 2 and $M$ inclusive, the integrability condition $\left[\mu^{\alpha}, v\right]$ is a consequence of conditions $[1, v-1],[1, v],[1, v+1]$ and $[2, v]$. (Of course, we omit $[1, v+1]$ from this list if $v=M$.)

Summary. The following table lists the important equations which have appeared in this chapter together with sufficient conditions for their validity:

(1) the B-conditions: $\left[\begin{array}{ll}B & 1\end{array}\right],\left[\begin{array}{ll}B & 2\end{array}\right],\left[\begin{array}{ll}B & 3\end{array}\right]$ if the basis beins $G^{\alpha}{ }_{\left(i_{w}\right)}$ are a basis of class $C^{1}$ and $r \geqq 3$.

(2) the A-conditions: [A 1 1], [ [ $\left.\begin{array}{ll}A & 2\end{array}\right]$ if $r \geqq 2$.

(3) the $R$-conditions: $\left[\begin{array}{ll}R & 1\end{array}\right],\left[\begin{array}{ll}R & 2\end{array}\right],\left[\begin{array}{ll}R & 3\end{array}\right]$ if the basis beins $G^{\alpha}{ }_{\left(i_{w}\right)}$ are a basis of class $C^{1}$ and $r \geqq 3$.

(4) the D-conditions: $\left[\begin{array}{ll}D & 1\end{array}\right],\left[\begin{array}{ll}D & 2\end{array}\right]$ if the basis beins $G_{\left({ }_{\left(i_{w}\right)}\right.}$ are a basis of class $C^{1}$ and $r \geqq 3$.

(5) the integrability conditions of the extended conformal Frenet equations:

$(\alpha)[0,2],[w, v] ; w=1,2, \cdots, M, v=1,2, \cdots, M, \infty, v \geqq w$, if the basis beins $G^{\alpha}{ }_{\left(i_{w}\right)}$ are a basis of class $C^{2}$ and $r \geqq 4$.

(B) $[\psi],\left[\psi_{i}\right]$ if $r \geqq 5$. If $n>3$, conditions $\left[\psi_{i}\right]$ are a consequence of $[1,1]$ and $[1,2]$.

$(\gamma)\left[\mu^{\alpha}, u\right], u=2,3, \cdots, M, \infty$ if $r \geqq 5$. If $n>2$, conditions $\left[\mu^{\alpha}, u\right]$ are a consequence of $[1, v]$ and $[2, v]$.

If any $U^{\left(h_{w}\right)}{ }_{\left.{ }_{\left(i_{w}+1\right.}\right)}, U^{\left(h_{w-1}\right)}{ }_{\left.i_{w+1}\right)}$ occurring in any of the above equations is replaced by another which differs from it by an additive null solution, the equation remains unchanged and the additional tensors due to the presence of the null tensor must vanish. All of the sufficient conditions enumerated above are satisfied if $r$ is equal to or greater than the maximum of the integers 5, $M+2$. It also follows from previous sections and the work of this chapter, that if the $A, B, D, R$ and integrability conditions are valid on a $V_{n}$ of class $C^{3}$ in a $V_{m}$ of class $C^{4}$ then they are also valid in any $\bar{V}_{n}$ in $\bar{V}_{m}$ obtained from $V_{n}$ in $V_{m}$ by an allowable conformal transformation. We call the totality of $A$-conditions, $R$-conditions, $D$-conditions and the integrability conditions the fundamental equations of $V_{n}$.

If $V_{n}$ is a hypersurface, the following simpler set of equations is equivalent to the set which appears above for a general subspace:

(1) the $B$-conditions: $\left[B^{\prime} 1\right],\left[B^{\prime} 2\right]$ if $r \geqq 1$.

(2) the A-conditions: $\left[A^{\prime} 1\right],\left[A^{\prime} 2\right]$ if $r \geqq 2$.

(3) the R-conditions: $\left[R^{\prime} 1\right],\left[R^{\prime} 2\right]$ if $r \geqq 1$.

(4) the integrability conditions of the extended conformal Frenet equations:

$(\alpha)[0,2],[1,1],[1,2]$ if $r \geqq 4$.

(及) $[\psi],\left[\psi_{2}\right]$ if $r \geqq 5$.

( $\gamma)[\mu]$ if $r \geqq 4$.

If $V_{m}$ is a conformally euclidean space $\bar{R}_{m}$, the integrability conditions 
$[w, v]$ are true identically if $|w-v|>2$. In this case, the complete set of integrability conditions $[w, v]$ of the conformal Frenet equations are $[0,2]$, $[w, v]$ where $w=1,2, \cdots, M$ and $v=w, w+1, w+2$ subject to the inequality $v<M+1$. The remaining integrability conditions which are not identities are $[\psi],\left[\psi_{i}\right],\left[\mu^{\alpha}, u\right]$ where $u=2,3,4$. We note that all the indices in the fundamental equations of $V_{n}$ are surface indices if $V_{m}$ is an $\bar{R}_{m}$. Space indices occur only in the extended conformal Frenet equations and the $B$-conditions.

\section{Subspaces in a CONformally EuClidean SPACE $\bar{R}_{m}$}

The Riemannian geometry of subspaces $V_{n}$ in an enveloping Riemann space $V_{m}$ is based upon the various fundamental forms of $V_{n}$. But these fundamental forms are the basis for a complete characterization of $V_{n}$ only in euclidean spaces and in spaces of constant curvature. In these spaces, we have the fundamental theorem of the Riemannian geometry of subspacesthe congruence theorem: All subspaces $V_{n}$ in an $S_{m}$ with equal fundamental forms are congruent; that is, they may be made to coincide by a motion in $S_{m}$. In addition, it is only in these spaces of constant curvature that one may obtain an existence theorem $\left({ }^{44}\right)$ for subspaces $\left({ }^{45}\right)$. This theorem affirms the existence of subspaces in $S_{m}$ whose fundamental forms are any arbitrary preassigned differential forms. The only conditions on these forms are that the coefficients of the forms satisfy certain partial differential equations-the generalization of the Gauss-Codazzi equations.

The analogues of these two theorems in conformal differential geometry hold when the enveloping space is any conformally euclidean $\left.{ }^{46}\right)$ space $\bar{R}_{m}$. In this chapter, we derive various minor results which are then used to prove the existence theorem and fundamental (conformal equivalence) theorem of the conformal differential geometry of subspaces.

27. Symmetry relations. As a result of (23.11), which is derived from the conformal Frenet equations (20.7) and (20.8), it follows that

$$
{ }^{*} G^{\alpha}{ }_{\left(i_{w}\right):\left(l_{v}\right)}=G^{\alpha}{ }_{\left(i_{w}\right)\left(l_{v}\right)}, \quad w+v=1,2, \cdots, M,
$$

where ${ }^{*} G^{\alpha}{ }_{\left(i_{w}\right):\left(l_{v}\right)}$ denotes the projection of the space vectors $G^{\alpha}{ }_{\left(i_{w}\right):\left(l_{v}\right)}$ into $I_{w+v}$. The integrability conditions (22.1), (22.3) and (23.10) may be written as

$$
\begin{aligned}
G^{\alpha}:(j k)^{-} & =0, \\
G^{\alpha}{ }_{i:(j k)^{-}} & \equiv-D^{\alpha}{ }_{i j k}+\frac{1}{(n-1)} G^{(02)} G_{i(k} G^{\alpha}{ }_{i)^{-}\left(o_{2}\right)}\left(\bmod I_{12}\right),
\end{aligned}
$$

(44) For a statement and proof of various forms of this existence theorem, see [3, chapter 9], $[10]$ and $[13,813]$.

${ }^{(45)}$ An exception to this statement occurs if $n=1$ (curve). In this case, there is an existence theorem for a curve in any enveloping space $V_{m}$.

(16) Since any space which is conformal to $S_{m}$ is also an $\bar{R}_{m}$, the analogy between Riemannian and conformal geumetry is complete as regards the enveloping space in which one may obtain an existence theorem and an equivalence theorem. 
(27.4) $\quad G^{\alpha}{ }_{\left(i_{u}\right):(j k)^{-}} \equiv-D^{\alpha{ }_{\beta j k} G^{\beta}{ }_{\left(i_{u}\right)}\left(\bmod I_{12} \ldots u\right),} u=2,3, \cdots, M-2$, after account is taken of (16.9), (16.10) and (27.1). If the space vectors in (27.2), (27.3) and (27.4) are projected into $I_{2}, I_{3}$ and $I_{u+2}$ respectively, it follows from (27.1) that

$$
\begin{aligned}
G^{\alpha}{ }_{(j k)^{-}} & =0, \\
G^{\alpha}{ }_{i(j k)^{-}} & =-{ }^{*} D^{\alpha_{i j k}}+\frac{1}{(n-1)} G^{(02)} G_{i(k} G^{\alpha}{ }_{i)^{-}\left(g_{2}\right)}, \\
G^{\alpha}{ }_{\left(i_{u}\right)(j k)^{-}} & =-{ }^{*} D^{\alpha_{\beta i k}} G^{\beta}{ }_{\left(i_{u}\right)}, \quad u=2,3, \cdots, M-2,
\end{aligned}
$$

where ${ }^{*} D_{\alpha \beta j k} G^{\beta}{ }_{\left(i_{u}\right)}$ is the projection of $D^{\alpha}{ }_{\beta j k} G^{\beta}{ }_{\left(i_{u}\right)}$ in $I_{u+2}$. It follows from (27.1) and (27.5) that

$$
G^{\alpha}{ }_{(i k)^{-}\left(l_{v}\right)}=0, \quad . v=0,1,2, \cdots, M-2 .
$$

This means that the basis beins $G^{\alpha}{ }_{\left(i_{w}\right)}$ are symmetric in the first two covariant indices $i_{1}, i_{2}$.

We now assume that $V_{m}$ is a conformally euclidean space $\bar{R}_{m}$. Then $C^{\alpha}{ }_{\beta \gamma \delta} \equiv 0$, so that $D^{\alpha}{ }_{\beta j k} \equiv D^{\alpha_{i j k}} \equiv 0$. Then, from (27.1) and (27.6), we find that

$$
G^{\alpha}{ }_{i(j k)^{-}\left(l_{v}\right)}=\frac{1}{(n-1)} G^{\left(\sigma_{2}\right)} G_{i(k} G^{\alpha}{ }_{j)^{-}\left(g_{2}\right)\left(l_{v}\right)}, \quad v=0,1,2, \cdots, M-3,
$$

since ${ }^{*} D^{\alpha_{i j k}}=0$. These equations show that, in general, the basis beins $G^{\alpha}{ }_{\left(i_{w}\right)}$ are not symmetric in the second and third covariant indices. It is readily seen from (27.9) that the necessary and sufficient condition that

$$
G^{\alpha}{ }_{i(j k)^{-}\left(l_{v}\right)}=0,
$$

$$
v=0,1,2, \cdots, M-3,
$$

is that, for the same value of $v$,

$$
G^{\left(o_{2}\right)} G^{\alpha_{i\left(\theta_{2}\right)\left(l_{v}\right)}}=0 .
$$

Since ${ }^{*} D^{\alpha}{ }_{\beta j k}=0,(27.7)$ shows that

$$
G^{\alpha}{ }_{\left(i_{u}\right)(j k)^{-}}=0,
$$$$
u=2,3, \cdots, M-2,
$$

that is, $G^{\alpha}{ }_{\left({ }_{i u}\right) j k}$ is symmetric in the indices $j, k$. In what follows, we prove that (27.13)

$$
\begin{array}{r}
G^{\alpha}{ }_{\left(i_{w}\right)} \text { is symmetric in the last }(w-2) \text { indices } i_{3}, i_{4}, \cdots, i_{w}, \\
w=4,5, \cdots, M .
\end{array}
$$

The proof depends upon mathematical induction. We suppose that $G^{\alpha}{ }_{\left(i_{0}\right)}$ is symmetric in the last $v-2$ covariant indices, where $v$ is some fixed integer between 4 and $M-1$ inclusive. According to (27.12), this is true if $v=4$. We now establish that $G^{\alpha}{ }_{\left(i_{v}+1\right)}$ must be symmetric in the last $(v-1)$ indices. From (27.1), it follows that

$$
{ }^{*} G^{\alpha}{ }_{\left(i_{v}-1\right) j: k}=G^{\alpha}{ }_{\left(i_{v}-1\right) i k}
$$


In accordance with the hypothesis of the induction, $G^{\alpha}{ }_{\left(i_{0}-1\right)} j$ is symmetric in the indices $i_{3}, i_{4}, \cdots, i_{v-1}, j$. Hence, from (27.14), this symmetry property is also enjoyed by the tensor $G^{\alpha}{ }_{\left(i_{v}-1\right) j k}$. But it has been shown that $G^{\alpha}{ }_{\left(i_{v}-1\right) j k}$ is symmetric in the indices $j, k$. Therefore $G^{\alpha}{ }_{\left(i_{v}-1\right) j k}$ is symmetric in the last $(v-1)$ indices or, using another notation, $G^{\alpha}{ }_{\left(i_{v+1}\right)}$ is symmetric in the indices $i_{3}, i_{4}, \cdots, i_{v+1}$. This completes the proof of the symmetry property stated in (27.13).

Incidentally, the above argument also shows that if $G^{\alpha}{ }_{\left(i_{v}\right)}$ is symmetric in the indices $i_{2}, i_{3}$ this is also true for the basis bein $G^{\alpha}{ }_{\left(i_{v+1}\right)}$. If account is taken of (27.1), (27.8) and (27.13), it follows from this statement that if $G^{\alpha}{ }_{\left(i_{v}\right)}(v>2)$ is symmetric in the indices $i_{2}, i_{3}$, then the basis beins

$$
G^{\alpha}{ }_{\left(i_{v}\right)}, G^{\alpha}{ }_{\left(i_{v+1}\right)}, G^{\alpha}{ }_{\left(i_{v+2}\right)}, \cdots, G^{\alpha}{ }_{\left(i_{M}\right)}
$$

enjoy symmetry in all their covariant indices and are symmetric covariant tensors. This occurs if and only if $G^{i_{2} i_{3}} G^{\alpha}{ }_{\left(i_{v}\right)}=0$. In this case, according to (27.11),

$$
G^{i_{a} i_{b} G^{\alpha}}{ }_{\left(i_{u}\right)}=0 \text {, }
$$

where $a, b$ are any integers between 1 and $u$.

This is similar to the equations

$$
G^{i_{1} i_{2}} G^{\alpha}{ }_{\left(i_{w}\right)}=0,
$$$$
w=2,3, \cdots, M,
$$

which follow from (7.11) [equivalent to (21.5)] and (27.1) and hold for all basis beins $G^{\alpha}{ }_{\left(i_{w}\right)}$ regardless of whether the $G^{\alpha}{ }_{\left(i_{w}\right)}$ are symmetric in $i_{2}, i_{3}$ and in any enveloping space $V_{m}$, not necessarily an $\bar{R}_{m}$.

The symmetry properties of the basis beins $G^{\alpha}{ }_{\left(i_{w}\right)}$ induce similar properties in the conformal measure tensors $B_{\left(i_{w}\right) \mid\left(h_{w}\right)}$. It follows directly from (21.2), (27.8), (27.9) and (27.13) that

$$
\begin{array}{r}
B_{(j k)-\left(l_{v}\right) \mid\left(h_{v+2}\right)}=0, \quad v=0,1,2, \cdots, M-2, \\
B_{i(j k)^{-}\left(l_{v}\right) \mid\left(h_{v+3}\right)}=\frac{1}{(n-1)} G^{\left(g_{2}\right)} G_{i(k} B_{j)^{-}\left(g_{2}\right)\left(l_{v}\right) \mid\left(h_{v+3}\right),} \\
v=0,1,2, \cdots, M-3, \\
B_{\left(i_{w}\right) \mid\left(h_{w}\right)} \text { is symmetric in the last }(w-2) \text { indices of }\left(i_{w}\right): i_{3}, i_{4}, \cdots, i_{w}, \\
w=4,5, \cdots, M .
\end{array}
$$

In addition, as stated in (21.6),

$$
B_{\left(i_{w}\right) \mid\left(h_{w}\right)}=B_{\left(h_{w}\right) \mid\left(i_{w}\right)}, \quad w=1,2, \cdots, M .
$$

Furthermore, as in equations (27.10) and (27.11) of the discussion for the basis beins, the necessary and sufficient condition that

$$
B_{i(j k)^{-}\left(l_{v}\right) \mid\left(h_{v+8}\right)}=0, \quad v=0,1,2, \cdots, M-3,
$$

is that, for the same value of $v$, 


$$
G^{\left(o_{2}\right)} B_{i\left(o_{2}\right)\left(l_{v}\right) \mid\left(h_{v+3}\right)}=0 .
$$

If $B_{\left(i_{v}\right) \mid\left(h_{v}\right)}(v>2)$ is symmetric in the indices $i_{2}, i_{3}$ then the normal measure tensors $B_{\left(i_{v}\right) \mid\left(h_{v}\right)}, B_{\left(i_{v}+1\right) \mid\left(h_{v+1}\right)}, \cdots, B_{\left(i_{M}\right) \mid\left(h_{M}\right)}$ enjoy symmetry in all the $i$-indices and in all the $h$-indices; that is, these measure tensors are unaltered by any interchange among the i-indices or any interchange among the $h$-indices. This occurs if and only if $G^{i_{2} i_{3}} B_{\left(i_{v}\right) \mid\left(h_{v}\right)}=0$. In this case, as in (27.15),

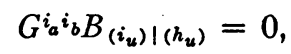

$u \geqq v$,

where $a, b$ are any integers between 1 and $u$. Even if $B_{\left(i_{w}\right) \mid\left(h_{w}\right)}$ is not symmetric in the indices $i_{2}, i_{8}, G^{i_{1} i_{2}} B_{\left(i_{w}\right) \mid\left(h_{w}\right)}=0, w=2,3, \cdots, M$, is true as is stated in (21.11). This is a special case of (27.22) and is true in any enveloping space $V_{m}$.

Finally we note that the symmetry properties of the $G^{\alpha}{ }_{\left(i_{w}\right)}$ also induce similar relations upon the covariant indices of the tensors $U^{\left(h_{w}\right)}{ }_{\left(i_{w}+1\right)}$, $U^{\left(h_{w-1}\right)}{ }_{\left(i_{w+1}\right)}$ which occur in the conformal Frenet equations. We state these relations in terms of the tensors $U_{\left(i_{w}+1\right) \mid\left(l_{w}\right)}$ and $U_{\left(i_{w}+1\right) \mid\left(l_{w-1}\right)}$ defined by means of the equations

$$
\begin{aligned}
U_{\left(i_{w+1}\right) \mid\left(l_{w}\right)} & =U^{\left(h_{w}\right)}{ }_{\left(i_{w+1}\right)} B_{\left(h_{w}\right) \mid\left(l_{w}\right),} \\
U_{\left(i_{w+1}\right) \mid\left(l_{w-1}\right)} & =U^{\left(h_{w-1}\right)}{ }_{\left(i_{w}+1\right)} B_{\left(h_{w-1}\right) \mid\left(l_{w-1}\right)} .
\end{aligned}
$$

It follows readily from (20.7), (21.2) and the symmetry relations for the $\Gamma_{\left({ }^{\prime} i_{w}\right)}$ that $U_{\left(i_{w+1}\right) \mid\left(l_{w}\right)}$ and $U_{\left(i_{w+1}\right) \mid\left(l_{w}-1\right)}$ are symmetric in the groups of indices $i_{1}, i_{2} ; i_{3}, i_{4}, \cdots, i_{w} ; l_{1}, l_{2} ;$ and either $l_{3}, l_{4}, \cdots, l_{w}$ or $l_{3}, l_{4}, \cdots, l_{w-1}$.

Also, according to (20.7),

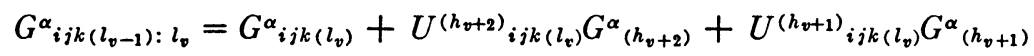

and

$$
\begin{aligned}
& {\left[\frac{1}{(n-1)} G^{\left(g_{2}\right)} G_{i k} G^{\alpha}{ }_{j\left(o_{2}\right)\left(l_{v-1}\right)}\right]: l_{v}} \\
& =\frac{1}{(n-1)} G^{(02)} G_{i k}\left[G^{\alpha}{ }_{j\left(o_{2}\right)\left(l_{v}\right)}+U^{\left(h_{v+2}\right)}{ }_{j\left(g_{2}\right)\left(l_{v}\right)} G^{\alpha}{ }_{\left(h_{v+2}\right)}+U^{\left(h_{v+1}\right)}{ }_{j\left(g_{2}\right)\left(l_{v}\right)} G^{\alpha}{ }_{\left(h_{v+1}\right)}\right] .
\end{aligned}
$$

Therefore we find from (21.1), (21.2), (27.9), (27.23) and the above equations that

$$
\begin{aligned}
& U_{i(j k)^{-}\left(l_{v}\right) \mid\left(h_{v+2}\right)}=\frac{1}{(n-1)} G^{\left(o_{2}\right)} G_{i(k} U_{j)^{-}\left(o_{2}\right)\left(l_{v}\right) \mid\left(h_{v+2}\right)}, \\
& v=0,1,2, \cdots, M-2, \\
& U_{i(j k)^{-}\left(l_{v}\right) \mid\left(h_{v+1}\right)}=\frac{1}{(n-1)} G^{\left(o_{2}\right)} G_{i(k} U_{j)^{-}\left(o_{2}\right)\left(l_{v}\right) \mid\left(h_{v+1}\right)}, \\
& v=0,1,2, \cdots, M-2 .
\end{aligned}
$$


We note that if $U_{\left(i_{w+1}\right) \mid\left(l_{w}\right)}$ and $B_{\left(h_{w}\right) \mid\left(l_{w}\right)}$ are known then $U^{\left(h_{w}\right)}{ }_{\left(i_{w+1}\right)}$ is determined except for an additive tensor $\theta^{\left(h_{w}\right)}{ }_{\left(i_{w}+1\right)}$ which obeys

$$
B_{\left(h_{w}\right) !\left(l_{w}\right)} \theta^{\left(h_{w}\right)}{ }_{\left(i_{w}+1\right)}=0 \text {. }
$$

Hence, for any fixed values of $i_{1}, i_{2}, \cdots, i_{w+1}, \theta^{\left(h_{w}\right)}{ }_{\left(i_{w+1}\right)}$ is a null solution and, according to $\$ 19$, also obeys the equations

$$
G_{\left(h_{w}\right)}^{\alpha} \theta_{\left(h_{w}\right)}=0 .
$$

This means that while the $U^{\left(h_{w}\right)}{ }_{\left(i_{w+1}\right)}$ are only fixed up to an additive null solution by the $U_{\left(i_{w+1}\right) \mid\left(l_{w}\right)}$ and $B_{\left(h_{w}\right) \mid\left(l_{w}\right)}$ the tensor $U^{\left(h_{w}\right)}{ }_{\left(i_{w+1}\right)} G^{\alpha}{ }_{\left(h_{w}\right)}$ which occurs in the conformal Frenet equations is uniquely determined by the $U_{\left(i_{w+1}\right) \mid\left(l_{w}\right)}$ and $G^{\alpha}{ }_{\left(h_{w}\right)}$. In a similar way, the $U_{\left(i_{w+1}\right) \mid\left(l_{w-1}\right)}$ and $B_{\left(h_{w}-1\right) \mid\left(l_{w}-1\right)}$ determine the $U^{\left(h_{w}-1\right)}{ }_{\left(i_{w+1}\right)}$ except for additive null solutions with respect to $B{ }_{\left(h_{w}-1\right) \mid\left(l_{w-1)}\right)}$ or $G^{\alpha}{ }_{\left(h_{w}-1\right)}$.

All of the symmetry properties obtained in this section are derived from the conformal Frenet equations, $B$-conditions and fundamental equations. Consequently the symmetry relations for $B_{\left(i_{w}\right) \mid\left(h_{w}\right)}, U_{\left(i_{w}\right) \mid\left(l_{w}-1\right)}$ and $U_{\left(i_{w}\right) \mid\left(l_{w}-2\right)}$ do not represent any new conditions. They may be derived directly from the fundamental equations.

28. Determination of $U^{\left(h_{w-1}\right)}{ }_{\left(i_{w+1}\right)}, U^{\left(h_{w}\right)}{ }_{\left(i_{w+1}\right)}, B_{\left(i_{w}\right) \mid\left(h_{w}\right)}$. In previous sections, it was shown that every subspace $V_{n}$ in $V_{m}$ which satisfies certain general conditions determines a set of conformal tensors. The tensors include the conformal measure tensors (19.8) and the deviation tensor $E_{i j}$ and are unchanged by conformal transformations of $V_{m}$. The components of these tensors satisfy the various algebraic and integrability conditions which were derived in chapter $\mathrm{V}$.

We are now in a position to begin the proof of a converse theorem which is true if the enveloping space $V_{m}$ is a conformally euclidean space $\bar{R}_{m}$. Briefly, this theorem states that subspaces $V_{n}$ exist in $\bar{R}_{m}$ whose conformal measure tensors and deviation tensor are any arbitrary tensors whose components satisfy the fundamental equations and that any such subspace is uniquely determined by a number of initial conditions. Indeed, we prove an even stronger theorem which may be stated loosely $\left({ }^{47}\right)$ as follows: $A V_{n}$ exists in $\bar{R}_{m}$ with preassigned conformal fundamental forms (19.11) whose coefficients satisfy certain algebraic and differential equations and this $V_{n}$ is uniquely determined by a number of initial conditions.

In its general outline, the derivation of these results is somewhat similar to that used to prove the corresponding existence theorem of Riemannian geometry $\left({ }^{48}\right)$. However, the details of the present proof involve enormously greater algebraic difficulties. As a result, there is only a very weak analogy between the details of the proofs of the preliminary theorems which lead up

(47) A precise statement of these theorems appears in Theorem 29.1 and Theorem 29.3.

$\left({ }^{48}\right)$ For example, compare the work of $\S \S 28,29$ of this paper with $[10, \S 4]$. 
to the existence theorems of conformal geometry and the corresponding theorems of Riemannian geometry. Since the enveloping $V_{m}$ is a conformally euclidean space, it is assumed that $C_{\alpha \beta \gamma \delta}=D_{\alpha \beta j k}=D_{\alpha i j k}=0$ in all the work of this section.

In this section, we develop a number of results of secondary interest which lead to a proof of the existence theorem. These results show that the tensors $U^{\left(h_{w}\right)}{ }_{\left(i_{w}+1\right)}, U^{\left(h_{w}-1\right)}{ }_{\left(i_{w+1}\right)}$ may be expressed in terms of the conformal measure tensors $B_{\left(i_{w}\right) \mid\left(l_{w}\right)}$ and of the deviation tensor $\left({ }^{49}\right) E_{i j}$ by means of the various equations derived in chapter $\mathrm{V}$ and finally that the $B_{\left(i_{w}\right) \mid\left(l_{w}\right)}$ in turn are uniquely determined by the symmetric conformal fundamental tensors $G_{\left(i_{w}\right)\left(l_{w}\right)}$. These results, which constitute the aim of the lengthy calculations of the present section, are stated in Theorem 28.1 and Theorem 28.2.

(1) Determination of the $U^{\left(h_{w-1}\right)}{ }_{\left(i_{w}+1\right)}$. According to (21.9) and the definition given by (27.23),

$$
U_{\left(i_{w+1}\right) \mid\left(l_{w-1}\right)}=-B_{\left(l_{w-1}\right) i_{w+1} \mid\left(i_{w}\right)}, \quad w=2,3, \cdots, M .
$$

[If $w=1, U_{\left(i_{2}\right) \mid\left(l_{0}\right)}=0$ by the convention of (20.8).] From the discussion at the end of $\$ 27$, this means that $B_{\left(l_{w}\right) \mid\left(i_{w}\right)}$ and $B_{\left(l_{w-1}\right) \mid\left(i_{w}-1\right)}$ fix the value of $U^{\left(h_{w}-1\right)}{ }_{\left(i_{w}+1\right)}$ except for the necessarily indeterminate additive null solution.

(2) Determination of the $U^{\left(h_{w}\right)}{ }_{\left.{ }_{i w+1}\right)}$. In the calculations that follow, the following notation is very useful. If a tensor expression $T$ is equal to a singlevalued $\left({ }^{50}\right)$ tensor function of

$$
\begin{gathered}
B_{i_{1} \mid l_{1}}, \quad \text { if } w=1, \\
E_{i l}, B_{i_{1} \mid l_{1}}, B_{\left(i_{2}\right) \mid\left(l_{2}\right)}, \cdots, B_{\left(i_{w}\right) \mid\left(l_{w}\right)}, \quad \text { if } w \geqq 2,
\end{gathered}
$$

we write

$$
T \stackrel{w}{\sim} 0
$$

to denote this fact. Similarly, if $T_{1}$ and $T_{2}$ are two tensor expressions and if $T_{1}$ differs from $T_{2}$ by a combination of the tensors (28.1), we write

$$
T_{1} \stackrel{w}{\sim} T_{2}
$$

Hence both (28.2) and (28.3) are equivalent to equations. We call each of them a $\sim$ wequation.

It is clear that (28.3) is equivalent to $T_{1}-T_{2} \sim^{w 0} 0$. Furthermore $T \sim^{w+1} 0$ and $T_{: 8} \sim{ }^{\infty} 0$ are both consequences of (28.2). Also, if

$$
T_{1} \stackrel{w}{\sim} 0, \quad T_{2} \stackrel{w}{\sim} 0
$$

(49) If $n>2$, the tensor $E_{i j}$ may be expressed in terms of $B_{i \mid l}$ and its derivatives and $B_{(i 2) \mid\left(l_{2}\right)}$ by means of (24.15). Hence, in this case, the $U$ s may be written as functions of the conformal measure tensors alone.

${ }^{\left({ }^{0}\right)}$ If $T$ is equal to $U^{\left(h_{w-1}\right)}{ }_{\left(i_{w+1}\right)}$ or $U^{\left(h_{w 0}\right)}{ }_{\left(i_{w+1}\right)}$ and is determined except for an additive null tensor, we also write (28.2). A similar remark applies to (28.35). 
then

$$
T_{1} \pm T_{2} \stackrel{w}{\sim} 0, \quad T_{1} T_{2} \stackrel{w}{\sim} 0
$$

so that, in some respects, (28.2) and (28.3) behave as though they were the equations $T=0$ and $T_{1}=T_{2}$ respectively. We also consider a generalization of the notion of symmetry for a tensor $T$. Let $i, j$ be two covariant (or contravariant) indices of $T$ and suppose that

$$
T_{i j}-T_{j i} \stackrel{w}{\sim} 0
$$

We call the kind of "symmetry" defined by (28.4) by the name $\sim^{w}$ symmetry and say that $T$ is a $\sim^{w}$ symmetric tensor in the indices $i, j$. Of course, ordinary symmetry implies $\sim$ w symmetry.

The first case in the determination of the $U^{\left(h_{w}\right)}{ }_{\left(i_{w+1}\right)}$ to be considered is $w=2$ since $U^{h_{1}}{ }_{\left(i_{2}\right)}=0$ by the convention stated in (20.8). We now find $U_{\left(i_{8}\right) \mid\left(l_{2}\right)}$. If $m=3$, this problem is solved by $(20.18)$. We therefore assume that $m$ exceeds 3 .

We introduce an abbreviated notation by means of the equations

$$
\begin{aligned}
\left\{\left(i_{w}\right)\left|\left(l_{w}\right)\right| j\right\} & =U_{\left(i_{w}\right) i \mid\left(l_{w}\right)} & & w>1, \\
\left\{i_{1} \mid\left(l_{2}\right)\right\} & =G^{h j}\left\{i_{1} h\left|\left(l_{2}\right)\right| j\right\}, & & \\
\left\{\left(i_{w-1}\right) \mid\left(l_{w}\right)\right\} & =G^{h i}\left\{i_{1} h j i_{2} i_{3} \cdots i_{w-2}\left|\left(l_{w}\right)\right| i_{w-1}\right\}, & & w>2, \\
{[l] } & =G^{h i}\left\{h \mid l_{j}\right\}, & & \\
{\left[\left(i_{w-1}\right)\left(l_{w-2}\right)\right] } & =G^{h i}\left\{\left(i_{w-1}\right) \mid l_{1} h j l_{2} l_{3} \cdots l_{w-2}\right\}, & & w>2 .
\end{aligned}
$$

From the symmetry properties of $\left\{\left(i_{2}\right)\left|\left(l_{2}\right)\right| j\right\}$ stated in $\$ 27$, it follows that

$$
\left\{i \mid(j k)^{-}\right\}=0 \text {. }
$$

Also, from equations (24.7),

$$
\{(i \mid j)-k\} \stackrel{2}{\sim} 0
$$

As a consequence of (28.6) and (28.7), it follows that $\{i \mid j k\}$ is a $\sim^{2}$ symmetric tensor in all its indices. We multiply (28.7) by $G^{i k}$ and sum on the repeated indices. Since

$$
G^{i k}\{j \mid i k\}=0,
$$

in accordance with (21.12), the resulting equations may be written as

$$
[j] \stackrel{2}{\sim} 0
$$

Now it follows from (21.10) with $w=2$ and (27.23) and (28.5) that

$$
\left\{\left(i_{2}\right)\left|\left(l_{2}\right)\right| j\right\}+\left\{\left(l_{2}\right)\left|\left(i_{2}\right)\right| j\right\}=B_{\left(i_{2}\right) \mid\left(l_{2}\right): j} .
$$

From (28.9), we obtain 
$(28.10)$

$$
\begin{aligned}
\left\{i_{1} i_{2}\left|l_{1} l_{2}\right| j\right\} & +\left\{l_{1} l_{2}\left|i_{1} i_{2}\right| j\right\}-\left\{l_{2 j}\left|i_{1} i_{2}\right| l_{1}\right\} \\
& -\left\{i_{1} i_{2}\left|l_{2 j}\right| l_{1}\right\}+\left\{i_{2} l_{1}\left|l_{2} j\right| i_{1}\right\}+\left\{l_{2} j\left|i_{2} l_{1}\right| i_{1}\right\} \\
& -\left\{j i_{1}\left|i_{2} l_{1}\right| l_{2}\right\}-\left\{i_{2} l_{1}\left|j i_{1}\right| l_{2}\right\}+\left\{l_{1} l_{2}\left|j i_{1}\right| i_{2}\right\} \\
& +\left\{j i_{1}\left|l_{1} l_{2}\right| i_{2}\right\} \stackrel{2}{\sim} 0 .
\end{aligned}
$$

Also, from the conformal Codazzi equations (22.20),

$$
\left\{i j^{-}\left|l_{1} l_{2}\right| k^{-}\right\}=\frac{1}{(n-1)} G_{i k^{-}}\left\{j^{-} \mid l_{1} l_{2}\right\} .
$$

If $(28.11)$ is substituted in $(28.10)$, we obtain

$$
\begin{aligned}
2\left\{i_{1} i_{2}\left|l_{1} l_{2}\right| j\right\} & +\frac{1}{(n-1)}\left[G_{l_{2}-}\left\{l_{1}-\mid i_{1} i_{2}\right\}+G_{i_{2} i_{1}}\left\{l_{1^{-}} \mid l_{2} j\right\}+G_{i i_{1}}-\left\{l_{2^{-}} \mid i_{2} l_{1}\right\}\right. \\
& \left.+G_{l_{1} i_{2}-}\left\{l_{2}-\mid j i_{1}\right\}+G_{i_{1} i_{2}}-\left\{j^{-} \mid l_{1} l_{2}\right\}\right] \stackrel{2}{\sim} 0 .
\end{aligned}
$$

When we make use of the $\sim^{2}$ symmetry of $\left\{j \mid l_{1} l_{2}\right\}$ these last equations become

$$
2\left\{i_{1} i_{2}\left|l_{1} l_{2}\right| j\right\}+\frac{2}{(n-1)}\left[G_{i_{1} i_{2}}\left\{l_{1} \mid l_{2} j\right\}-G_{l_{1} l_{2}}\left\{i_{1} \mid i_{2} j\right\}\right] \stackrel{2}{\sim} 0 .
$$

We multiply (28.12) by $G^{i_{1} i_{2}}$ and sum for $i_{1}, i_{2}$. If we make use of (21.11) and (28.5), we obtain

$$
n \cdot\left\{l_{1} \mid l_{2} j\right\}-G_{l_{1} l_{2}}[j] \stackrel{2}{\sim} 0 .
$$

In virtue of (28.8), these equations become $\left\{l_{1} \mid l_{2} j\right\} \sim^{20}$. If these last results are substituted in (28.12), we find that

$$
\left\{i_{1} i_{2}\left|l_{1} l_{2}\right| j\right\} \stackrel{2}{\sim} 0
$$

This means that $\left\{i_{1} i_{2}\left|l_{1} l_{2}\right| j\right\}$ or $U_{\left(i_{2}\right) j \mid\left(l_{2}\right)}$ may be expressed in terms of the tensors $E_{i l}, G_{i l}$ and $B_{\left(i_{2}\right) !\left(l_{2}\right)}$. Hence $U^{\left(h_{2}\right)}{ }_{\left(i_{8}\right)}$ is determined by these tensors up to an additive null solution.

We now proceed to a consideration of the general case $w>2$ which we establish by mathematical induction. For the hypothesis of the induction, we assume $\left({ }^{(51}\right)$ that the tensors

$$
U^{h_{1}}{ }_{\left(i_{3}\right)}, U^{\left(h_{2}\right)}{ }_{\left(i_{4}\right)}, \cdots, U^{\left(h_{w-2}\right)}{ }_{\left(i_{w}\right)} ; U^{\left(h_{2}\right)}{ }_{\left(i_{3}\right)}, U^{\left(h_{3}\right)}{ }_{\left(i_{4}\right)}, \cdots, U^{\left(h_{w-1}\right)}{ }_{\left(i_{w}\right)}
$$

may be expressed uniquely (except for additive null tensors) in terms of the tensors

$$
E_{i l}, G_{i l}, B_{\left(i_{2}\right) \mid\left(l_{2}\right)}, \cdots, B_{\left(i_{w-1}\right) \mid\left(l_{w-1}\right)}
$$

(51) This has already been shown for all the tensors of the first set in (28.14) as well as the first tensor $U^{\left(h_{2}\right)}{ }_{\left(i_{3}\right)}$ of the second set. 
In the sequel, we shall show that $U^{\left(h_{w}\right)}{ }_{\left(i_{w}+1\right)}$ is determined (except for an additive null solution) by the tensors $(28.15)$ and $B_{\left(i_{\infty}\right) \mid\left(h_{w}\right)}$. This is equivalent to proving that

$$
U_{\left(i_{w+1}\right) \mid\left(l_{w}\right)} \stackrel{w}{\sim} 0
$$

which is the form in which our result is established. The proof follows.

For the present, we assume that the tensors (28.14) are determined uniquely by the tensors (28.15). Later we consider the effect of changing any tensor of (28.14) by an additive null solution. According to the results of $\$ 27$, $\left\{\left(i_{w}\right)\left|\left(l_{w}\right)\right| j\right\}$ is symmetric in the groups of indices $i_{1}, i_{2} ; i_{3}, i_{4}, \cdots, i_{w}$; $l_{1}, l_{2}$ and $l_{3}, l_{4}, \cdots, l_{w}$. It also follows from (23.7), (23.17) and the hypothesis of the induction that

$$
\left\{\left(i_{w}\right)\left|\left(l_{w}\right)\right| j\right\}-\left\{\left(i_{w-1}\right) j\left|\left(l_{w}\right)\right| i_{w}\right\} \stackrel{w}{\sim} 0 .
$$

Also, from (21.10),

$$
\left\{\left(i_{w}\right)\left|\left(l_{w}\right)\right| j\right\}+\left\{\left(l_{w}\right)\left|\left(i_{w}\right)\right| j\right\} \stackrel{w}{\sim} 0 .
$$

It follows from (28.17) and (28.18) that

$$
\begin{aligned}
\left\{\left(i_{w}\right)\left|\left(l_{w}\right)\right| j\right\} & \stackrel{w}{\sim}-\left\{\left(l_{w}\right)\left|\left(i_{w}\right)\right| j\right\} \stackrel{w}{\sim}-\left\{\left(l_{w-1}\right) j\left|\left(i_{w}\right)\right| l_{w}\right\} \\
& \stackrel{w}{\sim}\left\{\left(i_{w}\right)\left|\left(l_{w-1}\right) j\right| l_{w}\right\} .
\end{aligned}
$$

As a consequence of (28.17) and (28.19) and the symmetry properties, $\left\{\left(i_{w}\right)\left|\left(l_{w}\right)\right| j\right\}$ is $\sim^{w}$ symmetric in the indices $i_{3}, i_{4}, \cdots, i_{w}, l_{3}, l_{4}, \cdots, l_{w}, j$. Now the integrability conditions (26.3) lead to the equations

$$
G^{i_{2} i_{3}} G_{i_{1}-i_{2}: i_{3} i_{4}}=0\left(\bmod I_{12}\right)
$$

when $V_{m}$ is an $\bar{R}_{m}$. Conformal differentiation of these equations and simplification by means of the conformal Frenet equations (20.7) yield the equations

$$
G^{i_{1} i_{2}} G_{i_{1}-i_{2}: i_{3} i_{1}-\cdots i_{w} j}^{\alpha_{0}} \equiv 0\left(\bmod I_{12} \cdots(w-1)\right) .
$$

Now according to (20.7) and easy mathematical induction,

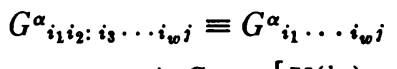

$$
\begin{aligned}
& +G^{\alpha}{ }_{\left(h_{w)}\right)}\left[U^{\left(h_{w 0}\right)_{i_{1}} \ldots i_{w} j}+\right.\text { combination of the tensors (28.14)] } \\
& \left(\bmod I_{12} \ldots_{(w-1)}\right) \text {. }
\end{aligned}
$$

We substitute (28.21) in (28.20) and multiply by $A_{\alpha \beta} G^{\beta}{ }_{\left(l_{w}\right)}$ and sum for $\alpha, \beta$. If use is made of (21.1), (21.2) as well as the definitions (27.23) and (28.5), we obtain

$$
G^{i_{1} i_{2}}\left\{i_{1}-i_{2} i_{3} i_{4}^{-} \ldots i_{w}\left|\left(l_{w}\right)\right| j\right\} \stackrel{w}{\sim} 0 .
$$

This result can be derived from the fundamental equations alone without using any equations involving space indices. Of course, (28.22) is equivalent to 


$$
\left\{i_{\overline{1}} i_{\overline{4}} \cdots i_{w} j \mid\left(l_{w}\right)\right\} \stackrel{w}{\sim} 0
$$

where $\left\{i_{1} i_{4} \cdots i_{w} j \mid\left(l_{w}\right)\right\}$ or, in other notation, $\left\{\left(i_{w-1}\right) \mid\left(l_{w}\right)\right\}$ is defined by (28.5). It follows from (28.23) and the $\sim$ w symmetry properties of $\left\{\left(i_{w}\right)\left|\left(l_{w}\right)\right| j\right\}$ stated in the preceding paragraph that $\left\{\left(i_{w-1}\right) \mid\left(l_{w}\right)\right\}$ is $\sim^{w}$ symmetric in the indices $i_{1}, i_{2}, i_{3}, \cdots, i_{w}, l_{3}, l_{4}, \cdots, l_{w}$.

From (28.18), we obtain

$$
G^{i_{2} i_{3}}\left\{\left(i_{w}\right)\left|\left(l_{w}\right)\right| j\right\}+\dot{G}^{i_{2} i_{3}}\left\{\left(l_{w}\right)\left|\left(i_{w}\right)\right| j\right\} \stackrel{w}{\sim} 0 .
$$

According to the conclusion of the preceding paragraph, the first tensor in (28.24) is $\sim^{w}$ symmetric in all indices except $l_{1}, l_{2}$. Consequently the second tensor $G^{\boldsymbol{i}_{2} i_{3}}\left\{\left(l_{w}\right)\left|\left(i_{w}\right)\right| j\right\}$ is also $\sim^{w}$ symmetric in these indices. Also $G^{l_{2} l_{3}}\left\{\left(l_{w}\right)\left|\left(i_{w}\right)\right| j\right\}$ is $\sim^{w}$ symmetric in all indices except $i_{1}, i_{2}$. It follows from these remarks that $G^{l_{2} l_{3}} G^{i_{2} i_{3}}\left\{\left(l_{w}\right)\left|\left(i_{w}\right)\right| j\right\}$ is $\sim^{w}$ symmetric in all the remaining indices. In the notation of $(28.5)$, this means that $\left[\left(i_{w-1}\right)\left(l_{w-2}\right)\right]$ is a $\sim^{w}$ symmetric tensor. If we multiply $(28.24)$ by $G^{l_{2} l_{3}}$ and sum on the repeated indices and use the fact that $\left[\left(i_{w-1}\right)\left(l_{w-2}\right)\right]$ is $\sim^{w}$ symmetric, we obtain

$$
\left[\left(i_{w-1}\right)\left(l_{w-2}\right)\right] \stackrel{w}{\sim} 0
$$

after replacing the indices $i_{1}, i_{4}, i_{5}, \cdots, i_{w}, j, l_{1}, l_{4}, l_{5}, \cdots, l_{w}$ by $i_{1}, i_{2}, i_{3}, \cdots$, $i_{w-1}, l_{1}, l_{2}, l_{3}, \cdots, l_{w-2}$.

Now (27.24) may be written as

$$
\left\{l_{1} l_{2}^{-} l_{3}^{-} \ldots l_{w}\left|\left(i_{w}\right)\right| j\right\}=\frac{1}{(n-1)} G_{l_{1} l_{3}^{-}}\left\{l_{2}^{-} l_{4} \cdots l_{w} j \mid\left(i_{w}\right)\right\} .
$$

From (28.18) and (28.26),

$$
\left\{\left(i_{w}\right)\left|l_{1} l_{2}^{-} l_{3}^{-} \cdots l_{w}\right| j\right\} \stackrel{w}{\sim} \frac{1}{(n-1)} G_{l_{1} l_{3}}\left\{l_{2}^{-} l_{4} \cdots l_{w} j \mid\left(i_{w}\right)\right\} .
$$

We multiply (28.27) by $G^{i_{2} i_{3}}$ and sum on repeated indices. This gives

$$
\left\{i_{1} i_{4} \cdots i_{w} j \mid l_{1} l_{2}^{-} l_{3}^{-} \cdots l_{w}\right\} \stackrel{w}{\sim} \frac{1}{(n-1)} G_{l_{1} l_{3}}\left[l_{2}^{-} l_{4} \cdots l_{w} j i_{1} i_{4} \cdots i_{w}\right]
$$

after using the definitions (28.5). According to (28.25) and a change of indices, these last equations become

$$
\left\{\left(i_{w-1}\right) \mid l_{1} l_{2}^{-} l_{3}^{-} \cdots l_{w}\right\} \stackrel{w}{\sim} 0
$$

so that $\left\{\left(i_{w-1}\right) \mid\left(l_{w}\right)\right\}$ is $\sim^{w}$ symmetric in the indices $l_{2}, l_{3}$. Since it has already been shown that $\left\{\left(i_{w-1}\right) \mid\left(l_{w}\right)\right\}$ is $\sim^{w}$ symmetric in all the indices except $l_{1}, l_{2}$ and is also symmetric in $l_{1}, l_{2}$, the above remark shows that $\left\{\left(i_{w-1}\right) \mid\left(l_{w}\right)\right\}$ is $\sim$ symmetric in all its indices.

We now consider a tensor expression suggested by (28.18). As a result of (28.18), 
(28.28)

$$
\begin{aligned}
& \left\{i_{1} \cdots i_{w}\left|l_{1} \cdots l_{w}\right| j\right\}+\left\{l_{1} \cdots l_{w}\left|i_{1} \cdots i_{w}\right| j\right\} \\
& -\left\{l_{2} \cdots l_{w} j\left|i_{1} \cdots i_{w}\right| l_{1}\right\}-\left\{i_{1} \cdots i_{w}\left|l_{2} \cdots l_{w} j\right| l_{1}\right\} \\
& +\left\{i_{2} \cdots i_{w} l_{1}\left|l_{2} \cdots l_{w} j\right| i_{1}\right\}+\left\{l_{2} \cdots l_{w} j\left|i_{2} \cdots i_{w} l_{1}\right| i_{1}\right\} \\
& -\left\{j i_{1} \cdots i_{w-1}\left|i_{w} l_{1} \cdots l_{w-1}\right| l_{w}\right\}-\left\{i_{w} l_{1} \cdots l_{w-1}\left|j i_{1} \cdots i_{w-1}\right| l_{w}\right\} \\
& +\left\{l_{1} \cdots l_{w}\left|j i_{1} \cdots i_{w-1}\right| i_{w}\right\}+\left\{j i_{1} \cdots i_{w-1}\left|l_{1} \cdots l_{w}\right| i_{w}\right\} \stackrel{w}{\sim} 0 .
\end{aligned}
$$

If use is made of the $\sim^{w}$ symmetry properties of $\left\{\left(i_{w}\right)\left|\left(l_{w}\right)\right| j\right\}$ and of (28.26), we find that (28.28) may be written as

$$
\begin{aligned}
& \left\{\left(i_{w}\right)\left|\left(l_{w}\right)\right| j\right\}+\frac{1}{(n-1)}\left[G_{l_{2} l_{3}}\left\{l_{1} l_{4} \cdots l_{w} j \mid i_{1} i_{2} \cdots i_{w}\right\}\right. \\
& -G_{i_{2} i_{3}}-\left\{i_{1}^{-} i_{4} \cdots i_{w} l_{1} \mid l_{2} \cdots l_{w} j\right\} \\
& +G_{l_{3} l_{4}}\left\{l_{2}^{-} l_{5} \cdots l_{w} j i_{1} \mid i_{2} i_{3} \cdots i_{w} l_{1}\right\}-\cdots \\
& +G_{j i 1^{-}}\left\{l_{w}^{-} i_{2} \cdots i_{w-1} \mid i_{w} l_{1} \cdots l_{w-1}\right\} \\
& \left.-G_{l_{1} l_{2}}-\left\{i_{w}-l_{3} \cdots l_{w} \mid j i_{1} \cdots i_{w-1}\right\}\right] \\
& +\left\{j i_{1} \cdots i_{w-1}\left|l_{1} \cdots l_{w}\right| i_{w}\right\} \stackrel{w}{\sim} 0 \text {. }
\end{aligned}
$$

Also, from (28.26),

$$
\begin{aligned}
\left\{j i_{1} \cdots i_{w-1}\left|l_{1} \cdots l_{w}\right| i_{w}\right\} & =\left\{\left(i_{w}\right)\left|\left(l_{w}\right)\right| j\right\} \\
+ & (1 /(n-1)) G_{i_{1} i_{2}}\left\{j-i_{3} \cdots i_{w} \mid l_{1} \cdots l_{w}\right\} .
\end{aligned}
$$

We substitute (28.30) in (28.29) and simplify using the fact that $\left\{\left(i_{w-1}\right) \mid\left(l_{w}\right)\right\}$ is a $\sim$ w symmetric tensor. The resulting equations are

$$
\begin{aligned}
2\left\{\left(i_{w}\right)\left|\left(l_{w}\right)\right| j\right\}+(2 /(n-1))\left[G_{i_{1} i_{2}}\left\{i_{3} \cdots i_{w} l_{1} \mid l_{2} \cdots l_{w} j\right\}\right. \\
-G_{l_{1} l_{2}}\left\{i_{w} l_{3} \cdots l_{w} \mid j i_{1} \cdots i_{w-1}\right\} \stackrel{w}{\sim} 0 .
\end{aligned}
$$

If (28.31) is multiplied by $G^{i_{1} i_{2}}$ and summed on repeated indices, we obtain

$$
n\left\{i_{3} \cdots i_{w} l_{1} \mid l_{2} \cdots l_{w} j\right\}-G_{l_{1} l_{2}}\left[i_{w} l_{3} \cdots l_{w} j i_{3} \cdots i_{w-1}\right] \stackrel{w}{\sim} 0
$$

after account is taken of (21.12) and (28.5). From these last equations and (28.25), it follows that

$$
\left\{\left(i_{w-1}\right) \mid\left(l_{w}\right)\right\} \stackrel{w}{\sim} 0
$$

after a change of notation. If (28.32) is substituted in (28.31), we find that

$$
\left\{\left(i_{w}\right)\left|\left(l_{w}\right)\right| j\right\} \stackrel{w}{\sim} 0
$$

which is equivalent to (28.16). The details of the proof show that $\left\{\left(i_{w}\right)\left|\left(l_{w}\right)\right| j\right\}$ 
is a rational function of the tensors (28.1) and their first conformal derivatives.

The above work disregarded the possible effect of changing any of the tensors (28.14) by additive null tensors $\left({ }^{52}\right)$. Now as noted in the summary at the conclusion of chapter $\mathrm{V}$, any change of this kind has no effect on any. of the equations used in our proof. This means that the value obtained for $U_{\left(i_{w+1}\right) \mid\left(l_{w}\right)}$ is not dependent upon the particular choice that we make for each $U$ in (28.14).

Consequently the $U^{\left(h_{w}\right)}{ }_{\left(i_{w+1}\right)}$ are uniquely determined by the tensors (28.1) except for necessarily indeterminate additive null solutions. This completes the proof by mathematical induction. The results of this section up to the present point may be summarized by the theorem:

TheOREM 28.1. The $\sim^{w}$ equations

$$
\begin{aligned}
U^{\left(h_{w-1}\right)}{ }_{\left(i_{w+1}\right)} & \stackrel{w}{\sim} 0, \quad \quad w=1,2, \cdots, M, \\
U^{\left(h_{w}\right)}{ }_{\left(i_{w+1}\right)} & \stackrel{w}{\sim} 0
\end{aligned}
$$

are consequences of the fundamental equations of $V_{n}$. The $U$ 's are uniquely determined by these $\sim^{w}$ equations except for additive null tensors.

(3) Determination of the $B_{\left(i_{w}\right)} \mid\left(l_{w}\right)$. The following conventions regarding notation are very useful for the calculations which appear in the remainder of this section. If a tensor expression $T$ is equal to a single-valued tensor function of

$$
\begin{array}{cl}
G_{i 1 l_{1}} & \text { if } w=1, \\
E_{i l}, G_{i_{1} l_{1}}, G_{\left(i_{2}\right)\left(l_{2}\right)}, \cdots, G_{\left(i_{w}\right)\left(l_{w}\right)} & \text { if } w \geqq 2,
\end{array}
$$

we write

$$
T \stackrel{w}{\simeq} 0
$$

to denote this fact. Similarly, if $T_{1}$ and $T_{2}$ are two tensor expressions and if $T_{1}$ differs from $T_{2}$ by a combination of the tensors (28.34), we write

$$
T_{1} \stackrel{\sim}{\simeq} T_{2} \text {. }
$$

According to these definitions, both (28.35) and (28.36) are equivalent to equations. We call each of them $\mathrm{a} \simeq{ }^{w}$ equation. The various remarks which appeared earlier in connection with the definitions of $\sim$ equations are also valid here in analogous form. In particular, if $i, j$ are two covariant (or contravariant) indices of $T$ and if $T_{i j}-T_{j i} \simeq{ }^{w} 0$ we say that $T$ is $a \simeq^{w}$ symmetric tensor in the indices $i, j$.

We begin by observing that $B_{i_{1} \mid l_{1}}\left(=G_{i_{1} l_{1}}\right)$ and $E_{i l}$ are themselves funda-

(52) The corresponding proof in the metric theory $[10, \S 4]$ should be amended to include remarks analogous to those made here. 
mental tensors. We now consider the problem of expressing $B_{\left(i_{2}\right) \mid\left(l_{2}\right)}$ in terms of the fundamental tensors. As a consequence of (19.12) and the symmetry properties of $B_{\left(i_{2}\right) \mid\left(l_{2}\right)}$, it follows that

$$
\begin{aligned}
3 G_{\left(i_{2}\right)\left(l_{2}\right)} & =B_{i_{1} i_{2} \mid l_{1} l_{2}}+B_{i_{1} l_{1} \mid i_{2} l_{2}}+B_{i_{1} l_{2} \mid i_{2} l_{1}} \\
& =3 B_{\left(i_{2}\right) \mid\left(l_{2}\right)}+B_{i_{1}\left(l_{1} \mid i_{2}\right) l_{2}}+B_{i_{1}\left(l_{2} \mid i_{2}\right)-l_{1}} .
\end{aligned}
$$

According to the conformal Gauss equations (22.13), $B_{i(k \mid j)-h}$ is a tensor function of $G_{i j}$ and $E_{i j}$. Hence, using the notation previously introduced,

$$
B_{i_{1}\left(l_{1} \mid i_{2}\right)^{-} l_{2}} \stackrel{2}{\simeq} 0, \quad B_{i_{1}\left(l_{2} \mid i_{2}\right)^{-} l_{1}} \stackrel{2}{\simeq} 0 .
$$

It follows from these equations and (28.37) that

$$
B_{\left(i_{2}\right) \mid\left(l_{2}\right)} \stackrel{2}{\simeq} 0 \text {. }
$$

We now proceed to a consideration of the general case $w>2$ which we establish by mathematical induction. For the hypothesis of the induction, we assume that the tensors

$$
E_{i l}, B_{i_{1} \mid l_{1}}, B_{\left(i_{2}\right) \mid\left(l_{2}\right)}, \cdots, B_{\left(i_{w-1}\right) \mid\left(l_{w-1}\right)}
$$

may be expressed uniquely in terms of the fundamental tensors

$$
E_{i l}, G_{i_{1} l_{1}}, G_{\left(i_{2}\right)\left(l_{2}\right)}, \cdots, G_{\left(i_{w-1}\right)\left(l_{w-1}\right)} \text {. }
$$

In the work that follows, we prove that $B_{\left(i_{w}\right) \mid\left(l_{w}\right)}$ is determined by the tensors (28.38) and $G_{\left(i_{w}\right)\left(l_{w}\right)}$; that is,

$$
B_{\left(i_{w}\right) \mid\left(l_{w}\right)} \stackrel{\boldsymbol{w}}{\simeq} 0
$$

We note that the hypothesis of the induction has been proved to be true if $w=1$ or $w=2$. From the hypothesis and Theorem 28.1, it follows that the tensors (28.14) are also determined (except for null solutions $\left({ }^{53}\right)$ ) by the fundamental tensors (28.38). Now, as a result of this remark, the hypothesis of the induction and equations (23.6) and (23.16), we find that

$$
U^{\left(h_{w-1}\right)}{ }_{\left(i_{w-1}\right)(j k)} \cdot B_{\left(h_{w-1}\right) \mid\left(l_{w-1}\right)} \stackrel{w}{\simeq} 0 .
$$

It follows from (21.9) that these equations are equivalent to

$$
B_{\left(i_{w-1}\right) k^{-}} \mid\left(l_{w-1}\right), \stackrel{w}{\simeq} 0 .
$$

Now, according to the results of $\S 27, B_{\left(i_{w}\right) \mid\left(l_{w}\right)}$ is symmetric in the groups of indices $i_{1}, i_{2} ; l_{1}, l_{2} ; i_{3}, i_{4}, \cdots, i_{w}$ and $l_{3}, l_{4}, \cdots, l_{w}$. It follows from (28.40) that $B_{\left(i_{w}\right) \mid\left(l_{w}\right)}$ is $\simeq^{w}$ symmetric in the indices $i_{3}, i_{4}, \cdots, i_{w}, l_{3}, l_{4}, \cdots, l_{w}$.

Equations (28.20) and (28.21) may be written in the form

(\$8) This lack of a unique determination for the tensors (28.14) may be disregarded in the present work for reasons which are stated in connection with the proof of Theorem 28.1. 


$$
\begin{aligned}
& G^{i_{2} i_{3}} G^{\alpha_{i_{1}}-i_{2}: i_{3} i_{4}}-\cdots i_{w} \equiv 0 \quad\left(\bmod I_{12} \cdots(w-2)\right), \\
& G^{\alpha}{ }_{i_{1} i_{2}: i_{3}} \cdots i_{w} \equiv G^{\alpha}{ }_{\left(i_{w}\right)}\left(\bmod I_{12} \cdots(w-1)\right) .
\end{aligned}
$$

We substitute (28.42) in (28.41) and multiply the result by $A_{\alpha \beta} G^{\beta}{ }_{\left(l_{w}\right)}$ and sum for $\alpha, \beta$. If use is made of (21.1) and (21.2), we obtain

$$
G^{(o)} B_{i_{1}-\left(g_{2}\right) i_{2}-\cdots i_{w-2} \mid\left(l_{w}\right)}=0
$$

after a change of indices. This result may be proved by means of the fundamental equations alone without using any equations involving space indices. It follows from (28.43) and the $\simeq^{w}$ symmetry properties of $B_{\left(i_{w}\right) \mid\left(l_{w}\right)}$ stated in the preceding paragraph that $G^{\left(o_{2}\right)} B_{i_{1}\left(g_{2}\right) i_{2} \cdots i_{w}-2 \mid\left(l_{w}\right)}$ is $\simeq^{w}$ symmetric in the indices $i_{1}, i_{2}, \cdots, i_{w-2}, l_{3}, l_{4}, \cdots, l_{w}$. From (21.6), we obtain

$$
G^{\left(g_{2}\right)} B_{i_{1}\left(o_{2}\right) i_{2} \cdots i_{w-2} \mid\left(l_{w}\right)}=G^{\left(g_{2}\right)} B_{\left(l_{w}\right) \mid i_{1}\left(o_{2}\right) i_{2} \cdots i_{w-2}} \text {. }
$$

Hence $G^{\left(\sigma_{2}\right)} B_{\left(l_{w}\right) \mid i_{1}\left(g_{2}\right) i_{2}} \cdots i_{w-2}$ is $\simeq^{w}$ symmetric in the indices $i_{1}, i_{2}, \cdots, i_{w-2}$, $l_{3}, l_{4}, \cdots, l_{w}$. It follows from these remarks that $G^{\left(a_{2}\right)} G^{\left(h_{2}\right)} B_{i_{1}\left(g_{2}\right) i_{2}} \cdots i_{i_{w-2}} \mid l_{1}\left(h_{2}\right) l_{2} \cdots l_{w-2}$ is $\sim^{w}$ symmetric in all its indices except $l_{1}$ and in all its indices except $i_{1}$ and hence is $\simeq^{w}$ symmetric in all its indices.

We shall use the following notation which is suggested by the $\simeq{ }^{w}$ symmetry properties derived above:

$$
\begin{aligned}
\left\{\left(i_{2}\right)\left|\left(l_{2}\right)\right|\left(h_{2 w-4}\right)\right\} & =B_{i_{1} i_{2} h_{1} \cdots h_{w-2} \mid l_{1} l_{2} h_{w-1} \cdots h_{2 w-4}}, \\
\left\{\left(l_{2}\right) \mid\left(i_{2 w-4}\right)\right\} & =G^{\left(o_{2}\right)} B_{i_{1}\left(g_{2}\right) i_{2} \cdots i_{w-2} \mid l_{1} l_{2} i_{w-1} \cdots i_{2 w-4}}, \\
{\left[\left(i_{2 w-4}\right)\right] } & =G^{\left(\sigma_{2}\right)} G^{\left(h_{2}\right)} B_{i_{1}\left(\sigma_{2}\right) i_{2} \cdots i_{w-2} \mid i_{w-1}\left(h_{2}\right) i_{w} \cdots i_{2 w-4}} .
\end{aligned}
$$

It follows that $\left\{\left(i_{2}\right)\left|\left(l_{2}\right)\right|\left(h_{2 w-4}\right)\right\}$ is $\simeq^{w}$ symmetric in the groups of indices $i_{1}, i_{2} ; l_{1}, l_{2} ; h_{1}, h_{2}, \cdots, h_{2 w-4}$ and that

$$
\left\{\left(i_{2}\right)\left|\left(l_{2}\right)\right|\left(h_{2 w-4}\right)\right\} \stackrel{w}{\simeq}\left\{\left(l_{2}\right)\left|\left(i_{2}\right)\right|\left(h_{2 w-4}\right)\right\} .
$$

Also, $\left\{\left(l_{2}\right) \mid\left(i_{2 w-4}\right)\right\}$ is $\simeq^{w}$ symmetric in $l_{1}, l_{2}$ and in $i_{1}, i_{2}, \cdots, i_{2 w-4}$ and $\left[\left(i_{2 w-4}\right)\right]$ is $\mathrm{a} \simeq^{w}$ symmetric tensor. We shall also write

$$
\begin{gathered}
{\left[\left(i_{2 w-6}\right)\right]=G^{\left(\sigma_{2}\right)}\left[g_{1} g_{2}\left(i_{2 w-6}\right)\right],} \\
{\left[\left(i_{2 w-8}\right)\right]=G^{\left(\sigma_{2}\right)}\left[g_{1} g_{2}\left(i_{2 w-8}\right)\right],} \\
\cdot \cdot \cdot \cdot \cdot \cdot \cdot \cdot \cdot \cdot \cdot . \\
{\left[\left(i_{2}\right)\right]=G^{\left(\sigma_{2}\right)}\left[g_{1} g_{2}\left(i_{2}\right)\right] .}
\end{gathered}
$$

It is clear that all the above tensors are $\simeq^{w}$ symmetric tensors.

We now derive several relationships which are used in the sequel to prove (28.39). According to (27.18) and (28.44),

$$
\begin{aligned}
\left\{\left(i_{2}\right)\left|\left(l_{2}\right)\right|\left(j_{2 w-4}\right)\right\}=\left\{i_{1} j_{1}\left|\left(l_{2}\right)\right| i_{2} j_{2} \cdots j_{2 w-4}\right\} \\
+(1 /(n-1)) G_{i_{1} j_{1}}\left\{\left(l_{2}\right) \mid i_{2}^{-} j_{2} \cdots j_{2 w-4}\right\} .
\end{aligned}
$$


If we multiply $(28.47)$ by $G^{\left(j_{2}\right)}$ and sum on the repeated indices, we obtain (28.48)

$$
\begin{aligned}
G^{j_{1} i_{2}}\left\{\left(i_{2}\right)\left|\left(l_{2}\right)\right|\left(j_{2 w-4}\right)\right\} \stackrel{w}{\simeq} & \frac{n}{(n-1)}\left\{\left(l_{2}\right) \mid i_{1} i_{2} j_{3} \cdots j_{2 w-4}\right\} \\
& -\frac{1}{(n-1)} G^{j_{1} j_{2}} G_{i_{1} i_{2}}\left\{\left(l_{2}\right) \mid\left(j_{2 w-4}\right)\right\}
\end{aligned}
$$

after account is taken of the $\simeq^{w}$ symmetry properties of $\left[\left(l_{2}\right) \mid\left(i_{2 w-4}\right)\right]$. Also, from (27.18), (28.44), (28.45) and (28.46), we obtain the equations

$$
\begin{aligned}
\left\{\left(l_{2}\right) \mid\left(j_{2 w-4}\right)\right\} \stackrel{w}{\simeq} & \left\{l_{1} j_{1} \mid l_{2} j_{2} \cdots j_{2 w-4}\right\} \\
& +(1 /(n-1)) G_{l_{1} j_{1}}\left[l_{2}^{-} j_{2} \cdots j_{2 w-4}\right] .
\end{aligned}
$$

If these last equations are multiplied by $G^{\left(j_{2}\right)}$ and summed for $j_{1}, j_{2}$, we obtain

$$
\begin{aligned}
G^{j_{1} j_{2}}\left\{\left(l_{2}\right) \mid\left(j_{2 w-4}\right)\right\} \stackrel{w}{\simeq} \frac{n}{(n-1)} & {\left[l_{1} l_{2} j_{3} \cdots j_{2 w-4}\right] } \\
& -\frac{1}{(n-1)} G_{l_{1} l_{2}}\left[j_{3} \cdots j_{2 w-4}\right]
\end{aligned}
$$

after account is taken of (28.46) and the $\simeq^{w}$ symmetry of the various tensors. Substitution of this last result in (28.48) leads to the equations

$$
\begin{aligned}
G^{j_{1} j_{2}}\left\{\left(i_{2}\right)\left|\left(l_{2}\right)\right|\left(j_{2 w-4}\right)\right\} \stackrel{w}{\simeq} & \frac{n}{(n-1)}\left\{\left(l_{2}\right) \mid i_{1} i_{2} j_{3} \cdots i_{2 w-4}\right\} \\
& -\frac{n}{(n-1)^{2}} G_{i_{1} i_{2}}\left[l_{1} l_{2} j_{3} \cdots j_{2 w-4}\right] \\
& +\frac{1}{(n-1)^{2}} G_{i_{1} i_{2}} G_{l_{1} l_{2}}\left[j_{3} \cdots j_{2 w-4}\right]
\end{aligned}
$$

We also note that if (28.47) is multiplied by $G^{i_{2} l_{2}}$ and summed on repeated indices, we obtain the equations

$$
\begin{aligned}
& G^{i_{2} l_{2}}\left\{\left(i_{2}\right)\left|\left(l_{2}\right)\right|\left(j_{2 w-4}\right)\right\} \stackrel{\mathcal{w}}{\simeq}\left\{i_{1} j_{1} \mid l_{1} j_{2} \cdots j_{2 w-4}\right\} \\
& \quad+\frac{1}{(n-1)} G_{i_{1} j_{1}}\left[l_{1} j_{2} \cdots j_{2 w-4}\right]-\frac{1}{(n-1)}\left\{i_{1} l_{1} \mid j_{1} \cdots j_{2 w-4}\right\} .
\end{aligned}
$$

Now, as in (28.49),

$$
\left\{i_{1} l_{1} \mid j_{1} \cdots j_{2 w-4}\right\}=\left\{i_{1} j_{1} \mid l_{1} j_{2} \cdots j_{2 w-4}\right\}+\frac{1}{(n-1)} G_{i_{1} j_{1}-}\left[l_{1}^{-} j_{2} \cdots j_{2 w-4}\right] .
$$

If this result is substituted in (28.51), we find that 
$(28.52)$

$$
\begin{aligned}
G^{i_{2} l_{2}}\left\{\left(i_{2}\right)\left|\left(l_{2}\right)\right|\left(j_{2 w-4}\right)\right\} \stackrel{w}{\simeq} \frac{(n-2)}{(n-1)}\left\{i_{1} j_{1} \mid l_{1} j_{2} \cdots j_{2 w-4}\right\} \\
+\frac{(n-2)}{(n-1)^{2}} G_{i_{1} j_{1}}\left[l_{1} j_{2} \cdots j_{2 w-4}\right]+\frac{1}{(n-1)^{2}} G_{i_{1} l_{1}}\left[j_{1} \cdots j_{2 w-4}\right] .
\end{aligned}
$$

Finally, if (28.49) is multiplied by $G^{\left(j_{2}\right)}$ and summed on $j_{1}, j_{2}$, we obtain

$$
\begin{aligned}
G^{j_{1} j_{2}}\left\{\left(l_{2}\right) \mid\left(j_{2 w-4}\right)\right\} \stackrel{w}{\simeq} & \frac{n}{(n-1)}\left[l_{1} l_{2} j_{3} \cdots j_{2 w-4}\right] \\
& -\frac{1}{(n-1)} G_{l_{1} l_{2}}\left[j_{3} \cdots j_{2 w-4}\right] .
\end{aligned}
$$

In the notation of the present section, (19.12) may be written as

$$
\sum\left\{\left(i_{2}\right)\left|\left(l_{2}\right)\right|\left(j_{2 w-4}\right)\right\} \stackrel{w}{\simeq} 0
$$

where the indices $i_{1}, i_{2}, l_{1}, l_{2}, j_{1}, \cdots, j_{2 w-4}$ in the summation assume all possible $(2 w)$ ! permutations of themselves. We now show, using the equations and $\simeq^{w}$ symmetry properties established in the preceding paragraphs, that (28.54) may be solved for the $B_{\left(j_{w}\right) \mid\left(k_{w}\right)}$. The proof is accomplished in a sequence of successive stages.

If account is taken of (28.47) and of the $\simeq^{w}$ symmetry properties of the tensors of (28.54), each of these tensors may be written as the fixed tensor $\left\{\left(i_{2}\right)\left|\left(l_{2}\right)\right|\left(j_{2 w-4}\right)\right\}$ plus a possible sum of terms like $\pm(1 /(n-1)) G_{\left(i_{2}\right)}$ $\left\{\left(l_{2}\right) \mid\left(j_{2 w-4}\right)\right\}$. Thus $(28.47)$ defines $\left\{i_{1} j_{1}\left|\left(l_{2}\right)\right| i_{2} j_{2} \cdots j_{2 w-4}\right\}$ in this manner. Similarly,

$$
\begin{aligned}
\left\{\left(i_{2}\right)\left|l_{1} j_{1}\right| l_{2} j_{2} \cdots j_{2 w-4}\right\} \stackrel{w}{\simeq} & \left\{\left(i_{2}\right)\left|\left(l_{2}\right)\right|\left(j_{2 w-4}\right)\right\} \\
& +\frac{1}{(n-1)} G_{l_{1} l_{2}}\left\{\left(i_{2}\right) \mid j_{1}-j_{2} \cdots j_{2 w-4}\right\} .
\end{aligned}
$$

Repeated application of (28.47) also leads to an analogous expression for $\left\{i_{1} l_{1}\left|i_{2} l_{2}\right|\left(j_{2 w-4}\right)\right\}$. It follows from these remarks that (28.54) may be written in the form

$$
c_{0}\left\{\left(i_{2}\right)\left|\left(l_{2}\right)\right|\left(j_{2 w-4}\right)\right\} \stackrel{w}{\simeq} \text { sum of terms like } \pm \frac{1}{(n-1)} G_{\left(i_{2}\right)}\left\{\left(l_{2}\right) \mid\left(j_{2 w-4}\right)\right\}
$$

where $c_{\theta}>0$, so that the problem of determining $\left\{\left(i_{2}\right)\left|\left(l_{2}\right)\right|\left(j_{2 w-4}\right)\right\}$ is reduced to the similar question for $\left\{\left(l_{2}\right) \mid\left(j_{2 w-4}\right)\right\}$. This completes the first stage of the proof.

If (28.54) is inultiplied by $G^{\left(i_{2}\right)}$ and summed for $i_{1}, i_{2}$, we obtain the $\simeq^{w}$ equations 


$$
\sum_{1} \stackrel{w}{\simeq} 0
$$

where $\sum_{1}$ is a sum of terms like

$$
\begin{aligned}
0,\left\{\left(l_{2}\right) \mid\left(j_{2 w-4}\right)\right\}, & G^{i_{1} i_{2}}\left\{\left(j_{2}\right)\left|\left(l_{2}\right)\right| i_{1} i_{2} j_{3} \cdots j_{2 w-4}\right\}, \\
& G^{i_{1} i_{2}}\left\{i_{1} l_{1}\left|i_{2} l_{2}\right|\left(j_{2 w-4}\right)\right\} .
\end{aligned}
$$

The first of these is obtained by the use of (21.11). In view of the $\simeq^{w}$ symmetry properties of $\left\{\left(i_{2}\right)\left|\left(l_{2}\right)\right|\left(j_{2 w-4}\right)\right\}$, these are the only possible types of terms which may occur in $\sum_{1}$. Now the second and third types listed in (28.56) may be changed by means of (28.50) and (28.52) respectively. Hence $\sum_{1}$ may be written as the sum of tensors like

$$
\begin{aligned}
\left\{\left(l_{2}\right) \mid\left(j_{2 w-4}\right)\right\} & \\
\frac{n}{(n-1)}\left\{\left(l_{2}\right) \mid\left(j_{2 w-4}\right)\right\} & -\frac{n}{(n-1)^{2}} G_{j_{1} j_{2}}\left[l_{1} l_{2} j_{3} \cdots j_{2 w-4}\right] \\
& +\frac{1}{(n-1)^{2}} G_{j_{1} j_{2}} G_{l_{1} l_{2}}\left[j_{3} \cdots j_{2 w-4}\right] \\
\frac{(n-2)}{(n-1)}\left\{l_{2} j_{1} \mid l_{1} j_{2} \cdots j_{2 w-4}\right\} & +\frac{(n-2)}{(n-1)^{2}} G_{l_{2} j_{1}}\left[l_{1} j_{2} \cdots j_{2 w-4}\right] \\
& +\frac{1}{(n-1)^{2}} G_{l_{1} l_{2}}\left[j_{1} \cdots j_{2 w-4}\right] .
\end{aligned}
$$

Since $n \geqq 2$, each of the terms (28.57) involves the tensors like $\left\{\left(l_{2}\right) \mid\left(j_{2 w-4}\right)\right\}$, $\left\{l_{2} j_{1} \mid l_{1} j_{2} \cdots j_{2 w-4}\right\}$ with non-negative coefficients. Also, if account is taken of (28.49) and the $\simeq^{w}$ symmetry properties of $\left\{\left(l_{2}\right) \mid\left(j_{2 w-4}\right)\right\}$, any tensor constructed from $\left\{\left(l_{2}\right) \mid\left(j_{2 w-4}\right)\right\}$ by a permutation of its indices may be written as the fixed tensor $\left\{\left(l_{2}\right) \mid\left(j_{2 w-4}\right)\right\}$ plus a possible sum of terms like $\pm(1 /(n-1)) G_{\left(l_{2}\right)}\left[\left(j_{2 w-4}\right)\right]$. It follows from these remarks that $(28.55)$ may be written as

$$
c_{1}\left\{\left(l_{2}\right) \mid\left(j_{2 w-4}\right)\right\} \stackrel{w}{\simeq} \text { sum of terms like } G_{\left(l_{2}\right)}\left[\left(j_{2 w-4}\right)\right] \text { with numerical coefficients }
$$

where $c_{1}>0$, so that the problem of determining $\left\{\left(l_{2}\right) \mid\left(j_{2 w-4}\right)\right\}$ is reduced to the similar question for $\left[\left(j_{2 w-4}\right)\right]$. This completes the second stage of the proof.

If (28.55) is multiplied by $G^{\left(l_{2}\right)}$ and summed for $l_{1}, l_{2}$, we obtain the $\simeq^{w}$ equations

$$
\sum_{2} \stackrel{w}{\simeq} 0
$$

Here $\sum_{2}$ is a sum of terms like those obtained by multiplying each of the tensors (28.57) by $G^{h_{1} h_{2}}$ where $h_{1}, h_{2}$ are any combination of the indices $l_{1}, l_{2}, j_{1}, j_{2}, \cdots, j_{2 w-4}$, and summing for the repeated indices. The first tensor 
in (28.57) may lead to terms like

$$
0,\left[\left(j_{2 w-4}\right)\right], \frac{n}{(n-1)}\left[\left(j_{2 w-4}\right)\right]-\frac{1}{(n-1)} G_{j_{1} j_{2}}\left[j_{3} \cdots j_{2 w-4}\right]
$$

after multiplication by $G^{l_{1} l_{2}}, G^{l_{1} j_{1}}$ and $G^{j_{1} j_{2}}$ respectively, and replacing $l$-indices by $j$ 's. Also, in the last case, we have made use of (28.53). The second tensor expression in (28.57) may lead to terms like

$(28.60)$

$$
\begin{gathered}
0, \frac{n(n-2)}{(n-1)^{2}}\left[\left(j_{2 w-4}\right)\right]+\frac{1}{(n-1)^{2}} G_{j_{1} j_{2}}\left[j_{3} \cdots j_{2 w-4}\right], \\
\quad \frac{n}{(n-1)}\left[\left(j_{2 w-4}\right)\right]-\frac{1}{(n-1)} G_{j_{1} i_{2}}\left[j_{3} \cdots j_{2 w-4}\right],
\end{gathered}
$$

$$
\begin{gathered}
\frac{n^{2}}{(n-1)^{2}}\left[\left(j_{2 w-4}\right)\right]-\frac{n}{(n-1)^{2}}\left(G_{j_{3} i_{4}}\left[j_{1} j_{2} j_{5} \cdots j_{2 w-4}\right]+G_{j_{1} j_{2}}\left[j_{3} \cdots j_{2 w-4}\right]\right) \\
+\frac{1}{(n-1)^{2}} G_{j_{1} j_{2} G_{j_{3} j_{4}}\left[j_{5} \cdots j_{2 w-4}\right]}
\end{gathered}
$$

after multiplication by $G^{l_{1} l_{2}}, G^{j_{1} l_{1}}, G^{l_{1} j_{3}}$ and $G^{j_{3} j_{4}}$ respectively and replacing $l$ 's by $j$ 's. In the last case, use has been made of (28.53). The third tensor expression in (28.57) may lead to terms like

$$
\begin{aligned}
& \frac{n}{(n-1)}\left[\left(j_{2 w-4}\right)\right], \quad\left[\left(j_{2 w-4}\right)\right], \\
& \frac{n(n-2)}{(n-1)^{2}}\left[\left(j_{2 w-4}\right)\right]+\frac{1 .}{(n-1)^{2}} G_{j_{1} j_{2}}\left[j_{3} \cdots j_{2 w-4}\right]
\end{aligned}
$$

after multiplication by $G^{l_{1} l_{2}}, G^{l_{1} j_{1}}$ and $G^{j_{1} j_{2}}$ respectively and replacing the $l^{\prime}$ s by $j$ 's. Now the tensors in (28.57) are $\simeq^{*}$ equivalent to those in (28.56). The $\simeq^{*}$ symmetry properties of the various tensors in (28.56) show that we have considered every possible product of one of these terms by $G^{h_{1} h_{2}}$ in deriving (28.59), (28.60) and (28.61). Hence $\sum_{2}$ may be written as a sum of terms like those which appear in (28.59), (28.60) and (28.61).

Since $n \geqq 2$, each of these terms involves the tensor $\left[\left(j_{2 w-4}\right)\right]$ with nonnegative coefficients. Since $\left[\left(j_{2 w-4}\right)\right]$ is a $\simeq^{w}$ symmetric tensor, it follows from the remarks of this and the preceding paragraphs that (28.58) may be written as

$$
c_{2}\left[\left(j_{2 w-4}\right)\right] \stackrel{w}{\simeq} \text { sum of terms like } G_{j_{2 w-5} j_{2 w-4}}\left[\left(j_{2 w-6}\right)\right] \text { with numerical coefficients }
$$

where $c_{2}>0$, so that the problem of finding $\left[\left(j_{2 w-4}\right)\right]$ is reduced to a similar one for $\left[\left(j_{2 w-6}\right)\right]$. This completes the third stage of the proof.

We may now multiply (28.58) by $G^{j_{2 w-6} j_{2 w-4}}$ and sum on repeated indices. 
In this way we obtain

$$
\sum_{3} \stackrel{w}{\simeq} 0
$$

Here $\sum_{3}$ is a sum of terms like those obtained by multiplying each of the tensors of (28.59), (28.60) and (28.61) by $G^{h_{1} h_{2}}$ where $h_{1}, h_{2}$ are any combination of the indices $j_{1}, j_{2}, \cdots, j_{2 w-4}$ and summing for repeated indices. All of these terms involve only non-negative coefficients except possibly the terms which arise from

$$
\begin{gathered}
\frac{n}{(n-1)}\left[\left(j_{2 w-4}\right)\right]-\frac{1}{(n-1)} G_{j_{1} j_{2}}\left[j_{3} \cdots j_{2 w-4}\right], \frac{n^{2}}{(n-1)^{2}}\left[\left(j_{2 w-4}\right)\right] \\
-\frac{n}{(n-1)^{2}}\left(G_{i_{3} i_{4}}\left[j_{1} j_{2} j_{5} \cdots j_{2 w-4}\right]+G_{j_{1} j_{2}}\left[j_{3} \cdots j_{2 w-4}\right]\right) \\
+\frac{1}{(n-1)^{2}} G_{j_{1} j_{2}} G_{j_{3} j_{4}}\left[j_{5} \cdots j_{2 w-4}\right] .
\end{gathered}
$$

Now the first of these expressions can only lead to terms like zero or like

$$
\frac{n}{(n-1)}\left[\left(j_{2 w-6}\right)\right]-\frac{1}{(n-1)} G_{i_{1} i_{2}}\left[j_{3} \cdots j_{2 w-6}\right]
$$

which we obtain by multiplying by $G^{j_{1} j_{2}}$ and $G^{j_{1} j_{3}}$ respectively and then changing our indices somewhat. The second of the expressions in (28.63) can only lead to terms like zero or like the expressions

$$
\begin{aligned}
& \frac{n(n-2)}{(n-1)^{2}}\left[\left(j_{2 w-6}\right)\right]+\frac{1}{(n-1)^{2}} G_{j_{1} i_{2}}\left[j_{3} \cdots j_{2 w-6}\right] \\
& \frac{n}{(n-1)}\left[\left(j_{2 w-6}\right)\right]-\frac{1}{(n-1)} G_{j_{1} j_{2}}\left[j_{3} \cdots j_{2 w-6}\right] \\
& \frac{n^{2}}{(n-1)^{2}}\left[\left(j_{2 w-6}\right)\right]-\frac{n}{(n-1)^{2}}\left(G_{j_{3} j_{4}}\left[j_{1} j_{2} j_{5} \cdots j_{2 w-6}\right]+G_{j_{1} i_{2}}\left[j_{3} \cdots j_{2 w-6}\right]\right) \\
& +\frac{1}{(n-1)^{2}} G_{j_{1} j_{2}} G_{j_{3} j_{4}}\left[j_{5} \cdots j_{2 w-6}\right]
\end{aligned}
$$

which we obtain by multiplying by $G^{j_{1} j_{2}}, G^{j_{1} j_{3}}, G^{j_{1 j} j_{5}}$ and $G^{j_{5 j} j_{6}}$ respectively and then changing some of the indices. In all the tensor expressions of (28.64) and (28.65), the coefficients of $\left[\left(j_{2 w-6}\right)\right]$ are non-negative.

It is clear from the above remarks that (28.62) may be written(54) as

$$
c_{3}\left[\left(j_{2 w-6}\right)\right] \stackrel{w}{\simeq} \text { tensor function of terms like } G_{j_{1} j_{2}},\left[\left(j_{2 w-8}\right)\right] \text { and }\left[\left(j_{2 w-10}\right)\right]
$$

(54) If $w=3$ or $w=4$, simple modifications of the present discussion are necessary. 
where $c_{3}>0$. This completes the fourth stage of the proof.

To find $\left[\left(j_{2 w-8}\right)\right]$, we multiply $(28.62)$ by $G^{j_{2 w-7 j} j_{2-6}}$ and sum on repeated indices. All the terms of the resulting $\simeq^{w}$ equations

$$
\sum_{4} \stackrel{w}{\simeq} 0
$$

have non-negative coefficients except possibly the terms which arise from the last two expressions of (28.65). But these expressions are exactly analogous to those of (28.63) which were considered in connection with (28.62). The previous discussion shows that the tensors $\left[\left(j_{2 w-8}\right)\right]$ which arise from these expressions have non-negative coefficients and consequently (28.66) may be solved for $\left[\left(j_{2 w-8}\right)\right]$ in terms of $\left[\left(j_{2 w-10}\right)\right]$, and so on. This completes the fifth stage of the proof.

This process may be continued until eventually, at the $(w+1)$ th stage of the proof, we reach $\mathrm{a} \simeq{ }^{w}$ equation which may be written as

$$
c_{w} G^{\left(j_{2}\right)}\left[\left(j_{2}\right)\right] \stackrel{w}{\simeq} 0
$$

with $c_{w}>0$. It follows from this equation that

$$
G^{\left(j_{2}\right)}\left[\left(j_{2}\right)\right] \stackrel{w}{\simeq} 0
$$

Since the solution for each of the tensors

$$
\begin{aligned}
G^{\left(j_{2}\right)}\left[\left(j_{2}\right)\right],\left[\left(j_{2}\right)\right], & {\left[\left(j_{4}\right)\right], \cdots,\left[\left(j_{2 w-4}\right)\right],\left\{\left(l_{2}\right) \mid\left(j_{2 w-4}\right)\right\}, } \\
& \left\{\left(i_{2}\right)\left|\left(l_{2}\right)\right|\left(j_{2 w-4}\right)\right\}
\end{aligned}
$$

is dependent only upon the solution for the preceding ones in the chain (28.68), it follows from (28.67) that

$$
\left\{\left(i_{2}\right)\left|\left(l_{2}\right)\right|\left(j_{2 w-4}\right)\right\} \stackrel{w}{\simeq} 0
$$

which is equivalent to (28.39). This completes that proof of the mathematical induction. The details of the proof show that $B_{\left(i_{w}\right) \mid\left(l_{w}\right)}$ is a rational function of the tensors (28.34) and their first conformal derivatives and of $K_{h i j k}$. The present proof as well as that of Theorem 28.1 only involves systems of linear algebraic equations. We may summarize the results of the above discussion and of Theorem 28.1 in the following theorem.

THEOREM 28.2. The $\simeq^{w}$ equations

$$
\begin{gathered}
B_{\left(i_{w}\right) \mid\left(l_{w}\right)} \stackrel{w}{\simeq} 0, \quad \quad w=1,2, \cdots, M, \\
U^{\left(h_{w-1}\right)}{ }_{\left(i_{w+1}\right)} \stackrel{\sim}{\simeq} 0, \quad U^{\left(h_{w}\right)}{ }_{\left(i_{w+1}\right)} \stackrel{w}{\simeq} 0
\end{gathered}
$$

are consequences of the fundamental equations of $V_{n}$ and of equation (19.12). The B's are uniquely determined by these $\simeq^{w}$ equations and the U's are uniquely determined except for additive null solutions. 
29. The existence theorem. We now prove the first form of the existence theorem:

TheOREM( $\left.{ }^{55}\right)$ 29.1. Let $\bar{R}_{m}$ be any conformally euclidean space of clqss $C^{4}$ and dimensionality $m>2$ and let $R$ be an open region of an $n$-dimensional number space $\left\{x^{i}\right\}$ of dimensionality $n>1$. Suppose that

$$
B_{i_{1} \mid l_{1}}, B_{\left(i_{2}\right) \mid\left(l_{2}\right)}, \cdots, B_{\left(i_{M}\right) \mid\left(l_{M}\right)}, E_{i l}
$$

are any functions of $x^{i}$ defined on $R$ so that the functions $B_{i_{1} \mid l_{1}}$ are of class $C^{2}$ and the others are of class $C^{1}$. Also, suppose that functions

(29.2) $U^{h_{1}}{ }_{\left(i_{3}\right)}, U^{\left(h_{2}\right)}{ }_{\left(i_{4}\right)}, \cdots, U^{\left(h_{M-1}\right)}{ }_{\left(i_{M+1}\right)} ; U^{\left(h_{2}\right)}{ }_{\left(i_{3}\right)}, U^{\left(h_{3}\right)}{ }_{\left(i_{4}\right)}, \cdots, U^{\left(h_{M}\right)}{ }_{\left.{ }_{\left(i_{M}+1\right.}\right)}$ of class $C^{1}$ on $R$ exist such that the fundamental equations formed with respect to (29.1) and (29.2) are true. Let $y^{\alpha}{ }_{0}$ be the coordinates of any point $\mathcal{P}$ of $\bar{R}_{m}$; $x^{i}{ }_{0}$ any fixed parameters belonging to $R ; \phi_{0}, \phi_{i 0}$ any $n+1$ real numbers; $G^{\alpha}{ }_{i 0}, G^{\alpha}{ }_{\left(i_{2}\right) 0}, \cdots, G^{\alpha}{ }_{\left(i_{M}\right) 0}, \mu^{\alpha}{ }_{0}$ any set of basis beins and any vector at $P$ which satisfy the boundary conditions when $x^{i}=x^{i}{ }_{0}$. Then there exists an $n$-dimensional Riemann space $V_{n}$ of class $C^{3}$ defined on some region of $R$ about $x^{i_{0}}$ which is imbedded in $\bar{R}_{m}$ by equations of the form

$$
y^{\alpha}=y^{\alpha}\left(x^{i}\right)
$$

and which has the respective functions (29.1) as its successive conformal measure tensors and deviation tensor respectively. For $x=x^{i}{ }_{0}, V_{n}$ passes through $P$ so that its moving conformal basis beins and mean curvature normal take the positions $G^{\alpha}{ }_{i 0}, G^{\alpha}{ }_{\left(i_{2}\right) 0}, \cdots, G^{\alpha}{ }_{\left({ }_{M}\right)}, \mu^{\alpha}{ }_{0}$ respectively and the values of the gauge function of $V_{n}$ and of its derivative with respect to $x^{i}$ at $\mathcal{P}$ are $\phi_{0}$ and $\phi_{i 0}$ respectively. Any other subspace with these properties will coincide with the $V_{n}$ defined by (29.3) in the common region of definition.

For the proof of this theorem, we consider the following system of partial differential equations in the unknown quantities (20.1):

$$
\begin{aligned}
\frac{\partial y^{\alpha}}{\partial x^{i_{1}}} & =G^{\alpha}{ }_{i_{1}}, \\
\frac{\partial G^{\alpha}{ }_{i_{1}}}{\partial x^{i_{2}}} & =G^{\alpha}{ }_{h} \Gamma_{i_{1} i_{2}}-G_{{ }_{i_{1}}} G^{\gamma_{i_{2}}} \Gamma_{\beta \gamma}^{\alpha}+G^{\alpha}{ }_{\left(i_{2}\right)},
\end{aligned}
$$

$$
\begin{aligned}
& \frac{\partial G^{\alpha}{ }_{{ }_{\left.i i_{w}\right)}}}{\partial x^{i_{w+1}}}=\sum_{v=1}^{w} G_{i_{1} \cdots i_{v-1} h i_{v+1} \cdots i_{w}} \Gamma_{i_{v} i_{w+1}}-G^{\beta}{ }_{\left(i_{w}\right)} G^{\gamma_{i_{w+1}}} \Gamma_{\beta \gamma}^{\alpha}+G^{\alpha}{ }_{\left(i_{w+1}\right)} \\
& +U^{\left(h_{w}\right)}{ }_{\left(i_{w+1}\right)} G^{\alpha}{ }_{\left(h_{w}\right)}+U^{\left(h_{w-1}\right)}{ }_{\left(i_{w+1}\right)} G^{\alpha}{ }_{\left(h_{w-1}\right)}, w=2,3, \cdots, M, \\
& \frac{\partial \psi}{\partial x^{i}}=\psi_{i},
\end{aligned}
$$

${ }^{(55)}$ Various modifications of this theorem appear in the remarks at the conclusion of the proof. 


$$
\begin{array}{r}
\frac{\partial \psi_{i}}{\partial x^{j}}=\psi_{h} \Gamma^{h}{ }_{i j}+\psi_{i} \psi_{j}+E_{i j}-a_{\alpha \beta} \mu^{\alpha} G^{\beta}{ }_{i j}+\frac{1}{(m-2)} \rho_{\alpha \beta} G^{\alpha}{ }_{i} G^{\beta}{ }_{j} \\
-\frac{1}{2}\left[e^{2 \psi} a_{\alpha \beta} \mu^{\alpha} \mu^{\beta}+G^{h k} \psi_{h} \psi_{k}+\frac{e^{2 \psi} \rho}{(m-1)(m-2)}\right] G_{i j},
\end{array}
$$

$$
\begin{aligned}
& \frac{\partial \mu^{\alpha}}{\partial x^{i}}=-\mu^{\beta} G^{\gamma_{i}} \Gamma^{\alpha}{ }_{\beta \gamma}+\frac{e^{-2 \psi}}{(n-1)}\left[G^{\alpha}{ }_{i h l} G^{h l}+U^{\left(g_{2}\right)}{ }_{i h l} G^{h l} G^{\alpha}{ }_{\left(o_{2}\right)}\right. \\
& +U^{o_{i h}} G^{h l} G^{\alpha}{ }_{g}+(n-1) G^{\alpha}{ }_{i h} G^{h} \psi_{l}-(n-1) l^{2 \psi} \mu^{\alpha} \psi_{i} \\
& \text { - }(n-1) a_{\beta \gamma} \mu^{\beta} G^{\gamma}{ }_{i h} G^{\alpha}{ }_{l} G^{h l}+G^{\alpha}{ }_{h} K^{h}{ }_{i}+(n-2) G^{\alpha}{ }_{h} E^{h}{ }_{i} \\
& \left.+G^{\alpha}{ }_{i} E-\frac{(n-1)}{(m-2)}\left(e^{2 \psi} a^{\alpha \beta}-G^{h k} G^{\alpha}{ }_{h} G^{\beta}{ }_{k}\right) \rho_{\beta \gamma} G^{\gamma}{ }_{i}\right] \text {. }
\end{aligned}
$$

Here $\Gamma^{i}{ }_{j k}$ and $K_{i j}$ are the Christoffel symbols of the second kind and the Ricci tensor formed with respect to $G_{i j}$ and $\Gamma^{\alpha}{ }_{\beta \gamma}$ is given by

$$
\Gamma_{\beta \gamma}^{\alpha}=\left\{\begin{array}{c}
\alpha \\
\beta \gamma
\end{array}\right\}+a^{\alpha}{ }_{\beta} \eta_{\gamma}+a^{\alpha}{ }_{\gamma} \eta_{\beta}-a_{\beta \gamma} a^{\alpha \delta} \eta_{\delta}
$$

where $\eta_{\gamma}$ is defined by

$$
\eta_{\gamma}=a_{\gamma \beta}\left[\mu^{\beta}-\psi_{h} e^{-2 \psi} G^{h k} G^{\beta}{ }_{k}\right] .
$$

The definition of $\Gamma^{\alpha}{ }_{\beta \gamma}$ in terms of the given tensors and the unknowns (20.1) is suggested by (8.8), (8.10), (8.11) and (13.10). We also define $A_{\alpha \beta}$ by means of the equations

$$
A_{\alpha \beta}=e^{-2 \psi} a_{\alpha \beta} .
$$

Of course, equations (29.4) are the extended conformal Frenet equations after account is taken of the definition of the conformal derivative.

To the system of equations (29.4), we adjoin the equations

$$
\begin{aligned}
a_{\alpha \beta} G^{\alpha}{ }_{\left(i_{w}\right)} G^{\beta}{ }_{\left(l_{v}\right)} & =0, \quad w \neq v, w, v=1,2, \cdots, M, \\
a_{\alpha \beta} G^{\alpha}{ }_{\left(i_{w}\right)} G^{\beta}{ }_{\left(l_{w}\right)} & =e^{2 \psi} B_{\left(i_{w}\right) \mid\left(l_{w}\right)}, \\
a_{\alpha \beta} \mu^{\alpha} G^{\beta}{ }_{j} & =0 .
\end{aligned}
$$

These equations constitute a set of algebraic boundary conditions which must be satisfied by the solutions of (29.4). In virtue of (29.6), it is clear that (29.7) are equivalent to the $B$-conditions.

The right members of equations (29.4) depend upon the known functions

$$
\Gamma^{i}{ }_{j k}(x), U_{\ldots} \cdots(x), G_{i j}(x), K_{i j}(x), E_{i j}(x), E(x)
$$

and the unknowns (20.1). These latter appear both explicitly and also implicitly in the functions 


$$
a_{\alpha \beta}(y),\left\{\begin{array}{c}
\alpha \\
\beta \gamma
\end{array}\right\}, \rho_{\alpha \beta}(y), \rho(y) \text {. }
$$

Actually, the $U$ 's are not given functions. The hypothesis of the theorem merely affirms the existence of at least one set of $U$ 's. But according to Theorem 28.1, any other set of $U$ 's differs from the first by null solutions which do not affect equations (29.4). Hence we may think of the $U$ 's as uniquely determined by the given functions (29.1).

According to the hypothesis, $a_{\alpha \beta}(y)$ is of class $C^{3}, G_{i j}(x)$ is of class $C^{2}$ and $E_{i j}(x)$ is of class $C^{1}$. Since $G_{i j}$ is of class $C^{2}, \Gamma^{i}{ }_{j k}$ is of class $C^{1}$ and $K_{h i j k}$ is of class $C^{0}$. However, since the conformal Gauss equations (22.13) (condition $[1,1])$ are satisfied, it follows from the hypothesis of the theorem that $K_{h i j k}$ are actually of class $C^{1}$ in the $x^{i}$. Hence each right member of (29.4) is of class $C^{1}$ in all the dependent variables (20.1) and in the $x^{i}$ for any $y^{\alpha}$ in the coordinate system; any $x^{i}$ in $R$; any $\psi, \psi_{i}$; any $G^{\alpha}{ }_{i}, G^{\alpha}{ }_{\left(i_{2}\right)}, \cdots, G^{\alpha}{ }_{\left({ }_{i M}\right)}, \mu^{\alpha}$. The equations obtained by partial differentiation of (29.7) and utilizing both (29.4) and the boundary conditions are the set of equations which we have called the $D$-conditions. Now, by hypothesis, both the $D$-conditions and the integrability conditions of (29.4) are true identically. The remarks of the last two paragraphs show that we may apply the fundamental existence theorem $\left({ }^{56}\right)$ for such a system of partial differential equations with boundary conditions.

According to this theorem, there exists a set of functions

$$
y^{\alpha}(x), G^{\alpha}{ }_{i_{1}}(x), \cdots, G^{\alpha}{ }_{\left(i_{M}\right)}(x), \mu^{\alpha}(x), \psi(x), \psi_{i}(x)
$$

of class $C^{1}$, satisfying the equations (29.4) and the boundary conditions (29.7) throughout the region of definition, which are defined in a sufficiently small region of $R$ about $x^{i_{0}}$ and which assume the values $y^{\alpha}{ }_{0}, G^{\alpha}{ }_{i 0}, \cdots, G^{\alpha}{ }_{\left(i_{M}\right) 0}$, $\mu_{0}^{\alpha},-\phi_{0},-\phi_{i 0}$ for $x=x^{i}{ }_{0}$. Any two solutions of (29.4) which have the same initial conditions for $x=x^{i}{ }_{0}$ are identical in their common region of definition.

Since the functions (29.8) are of class $C^{1}$, it follows from the equations of (29.4) which involve $\partial \psi_{i} / \partial x^{i}$ that $\partial \psi_{i} / \partial x^{j}$ is actually of class $C^{1}$ so that $\psi$ is of class $C^{3}$. Also, since $\mu^{\alpha}$ is of class $C^{1}, \Gamma^{\alpha}{ }_{\beta \gamma}$ is also of class $C^{1}$. If we now return to the equations of (29.4) which involve $\partial \mu^{\alpha} / \partial x^{i}$, we see that $\partial \mu^{\alpha} / \partial x^{i}$ is actually of class $C^{1}$ so that $\mu^{\alpha}$ and consequently $\Gamma^{\alpha}{ }_{\beta \gamma}$ are of class $C^{2}$. These remarks and the conformal Frenet equations of (29.4) show that the various basis biens $G^{\alpha}{ }_{\left(i_{w}\right)}$ are actually of class $C^{2}$. It follows from the special form of the first set of equations of (29.4) that the $y^{\alpha}(x)$ are of class $C^{3}$.

The hypothesis of the existence theorem assumes that real basis beins $G^{\alpha}{ }_{\left(i_{w}\right)}$ exist at $\mathbb{P}$ which satisfy the boundary conditions. Since the $B_{\left(i_{w}\right) \mid\left(l_{w}\right)}$ all satisfy the $R$-conditions, the existence of such real basis beins for any $x^{i}$

${ }^{(56)}$ The existence theorem which we are using is an easy modification (to allow boundary conditions) of one which appears in [11, p. 661]. 
in $R$ is guaranteed. Further, since $B_{i_{1} \mid l_{1}}$ is positive definite by condition [R 2], it follows from the second set of boundary conditions (29.7) with $w=1$ that the rank of the matrix $\left\|G^{\alpha}{ }_{i_{1}}\right\|$ is $n$ for $x^{i}$ in $R$. Consequently, according to the definition and discussion contained in $\$ 4$, the subspace defined by (29.3), where the $y^{\alpha}\left(x^{i}\right)$ are the unique solutions of (29.4), is an $n$-dimensional Riemann space $V_{n}$ of class $C^{3}$.

According to the first set of equations (29.4), $G^{\alpha}{ }_{i}$ spans the tangent vector space of $V_{n}$. Hence the Riemann metric tensor of $V_{n}$ is defined by

$$
g_{i j}=a_{\alpha \beta} G^{\alpha}{ }_{i} G^{\beta}{ }_{j} .
$$

A comparison of these equations with (29.7) for $w=1$ shows that

$$
e^{2 \psi} G_{i j}=g_{i j}
$$

where we have written $G_{i j}$ for $B_{i_{1 \mid l} l_{1}}$. It follows from these equations that

$$
\left\{\begin{array}{c}
i \\
j k
\end{array}\right\}=\Gamma^{i}{ }_{j k}+G^{i}{ }_{j} \psi_{, k}+G^{i} \psi_{, j}-G_{j k} G^{i} \psi_{, l}
$$

where

$$
\left\{\begin{array}{c}
i \\
j k
\end{array}\right\}
$$

are the Christoffel symbols of the second kind formed with respect to $g_{i j}$. Also, (5.3) is true for $V_{n}$ where $g^{\alpha}{ }_{i}(x)$ and $G^{\alpha}{ }_{i}(x)$ are identical functions. Hence it follows from (5.3) and the second set of equations (29.4) that

$$
G^{\alpha}{ }_{h}\left[\Gamma^{h}{ }_{i j}-\left\{\begin{array}{c}
h \\
i j
\end{array}\right\}\right]-G^{\beta}{ }_{i} G^{\alpha}{ }_{j}\left[\Gamma^{\alpha}{ }_{\beta \gamma}-\left\{\begin{array}{c}
\alpha \\
\beta \gamma
\end{array}\right\}\right]=g^{\alpha}{ }_{i j}-G^{\alpha}{ }_{i j} .
$$

If we simplify these equations, using (29.5), (29.9), (29.10) and the boundary conditions, we find that

$$
G^{\alpha}{ }_{i j}=g^{\alpha}{ }_{i j}-\mu^{\alpha} g_{i j}
$$

If we multiply these equations by $G^{i j}$ and sum on repeated indices, using the $A$-condition $[A 1$ ] (in the equivalent form of equation (7.11)) and (29.9), we obtain equations (5.9). Consequently the functions $\mu^{\alpha}(x)$ listed in (29.8) are the components of the mean curvature normal of $V_{n}$. It also follows from (29.11) that $G^{\alpha}{ }_{i j}(x)$ is the basis bein which spans the first conformal normal vector space of $V_{n}$. The relative conformal curvature $\Lambda$ of $V_{n}$ is defined by (6.12). If use is made of condition [ $A$ 2] (in the form of equation (7.12)), (29.6) and (29.9), we find that $\Lambda=e^{-\psi}$ so that $-\psi$ is the guage function.

As a result of the geometric interpretation which we have shown for the functions $\mu^{\alpha}(x), \psi(x), G^{\alpha}{ }_{i}(x)$ and $G^{\alpha}{ }_{i j}(x)$, it is clear from equations (29.5), (29.6) and (29.9) that $A_{\alpha \beta}(x), G_{i j}(x), \Gamma^{\alpha}{ }_{\beta \gamma}(x)$ and $\Gamma^{i}{ }_{j k}(x)$, defined in this section, are the conformal measure tensors and conformal coefficients of connec- 
tion of $\bar{R}_{m}$ and $V_{n}$ respectively. It is then immediate from the boundary conditions and the remaining equations $(29.4)$ that $G^{\alpha}{ }_{i_{1}}(x), G^{\alpha}{ }_{\left(i_{2}\right)}(x), \cdots, G^{\alpha}{ }_{\left({ }_{i M}\right)}(x)$ are the conformal basis beins of $V_{n}$ and that the given functions (29.1) are the successive conformal measure tensors and deviation tensor respectively of $V_{n}$.

The choice of initial conditions in the determination of $V_{n}$ makes it clear that for $x=x^{i}{ }_{0}, V_{n}$ passes through $P$ so that its moving conformal basis beins and mean curvature normal occupy the positions preassigned by the theorem and so that the gauge function of $V_{n}$ and its derivative with respect to $x^{i}$ at $\mathcal{P}$ are $\phi$ and $\phi_{i 0}$. This remark completes the proof of the theorem. We note that, in this proof, the theorem applies to a fixed coordinate and parameter system. Nevertheless, under coordinate transformations of class $C^{4}$ and parameter changes of class $C^{3}$, the solutions in one coordinate-parameter system will transform into the solutions in any other allowable system since the partial differential equations (29.4) may be written in the invariant form, tensor $=$ tensor. Finally, we remark that all the derivations of the various fundamental equations of $V_{n}$ are valid under the hypothesis of the existence theorem.

Remark 1. Since the $G^{\alpha}{ }_{\left({ }_{(w)}\right)}$ are all of class $C^{2}$, it follows from $(29.7)$ that the various conformal normal measure tensors $B_{\left(i_{2}\right) !\left(l_{2}\right)}, \cdots, B_{\left(i_{M}\right) !\left(l_{M}\right)}$ must be of class $C^{2}$. This means that if the complete hypothesis of Theorem 29.1 is satisfied (including the validity of the fundamental equations), the conformal measure tensors must all be of class $C^{2}$ instead of class $C^{\mathbf{1}}$.

Remark 2. If $B_{i_{1} \mid l_{1}}$ is assumed to be of class $C^{3}$ instead of class $C^{2}$, then the $\Gamma^{i}{ }_{j k}$ are of class $C^{2}$. Consequently it is seen from the special form of the first two sets of equations (29.4) that $y^{\alpha}\left(x^{i}\right)$ are of class $C^{4}$ in this case.

Remark 3. Suppose that the enveloping space is a euclidean space $R_{m}$ or any space of constant curvature $S_{m}$ and that we limit the conformal transformations to mappings between spaces of this kind only $\left({ }^{57}\right)$. Then the hypothesis of Theorem 29.1 may be weakened so that $R_{m}$ or $S_{m}$ is of class $C^{3}$ instead of class $C^{4}$ and the remainder of the theorem will hold as stated. For in this case, $\rho_{\alpha \beta \gamma \delta}$ is replaced by $k\left(a_{\alpha \gamma} a_{\beta \delta}-a_{\alpha \delta} a_{\beta \gamma}\right)$ where $k$ is the constant Riemann curvature of the space so that only the first derivatives of $a_{\alpha \beta}$ occur in equations (29.4).

Remark 4. According to Theorem 26.1, the conditions $\left[\mu^{\alpha}, u\right]$ may be omitted from the fundamental equations if $n>2$. According to Theorem 24.1, the conditions $\left[\psi_{i}\right]$ may be omitted from the fundamental equations if $n>3$. If $n>2$, the tensor $E_{i l}$ is defined in terms of $B_{\left(i_{2}\right) \mid\left(l_{2}\right)}$ and $G_{i l}$ by means of (24.15) and therefore is not an independent tensor.

By considering equations (20.12), (20.13), (20.14), (20.23) and the first

(57) These transformations are a first generalization of the inversive group of transformations of $R_{m}$ on itself. These transformations may be characterized by the fact that they map each geodesic circle of a space of constant curvature on a geodesic circle in the image space. 
two sets of equations (20.9) instead of equations (29.4), a somewhat simpler formulation may be given to the existence theorem for a hypersurface. As the methods parallel those used in the general case (except that Theorem 28.1 plays no role in the proof), we state this theorem without proof.

THEOREM 29.2 Let $\bar{R}_{m}$ be any conformally euclidean space of class $C^{4}$ and dimensionality $m>2$ and let $R$ be an open region of an $(m-1)$-dimensional number space $\left\{x^{i}\right\}$. Suppose that

$$
G_{i j} d x^{i} d x^{j}, \quad B_{i j} d x^{i} d x^{j}, \quad E_{i j} d x^{i} d x^{j}
$$

are any symmetric quadratic differential forms defined on $R$ of which the first is positive definite and so that the coefficients of these forms are of class $C^{2}, C^{2}$ and $C^{1}$ respectively. Also, suppose that the $A$-conditions $\left[A^{\prime} 1\right],\left[A^{\prime} 2\right]$ and the integrability conditions $[1,1],[1,2],\left[\psi_{i}\right],[\mu]$ are satisfied by these coefficients. Let $y^{\alpha}$ be the coordinates of any point $P$ of $\bar{R}_{m} ; x^{i}{ }_{0}$ any fixed parameters belonging to $R ; \mu_{0}, \phi_{0}, \phi_{i 0}$ any $(m+1)$ real numbers; $G^{\alpha}{ }_{i 0}, Z^{\alpha}{ }_{0}$ any basis bien and vector at $P$ which satisfy the boundary conditions $\left[B^{\prime} 1\right]$ and $\left[B^{\prime} 2\right]$ when $x=x^{i}{ }_{0}$. Then there exists an $(m-1)$-dimensional hypersurface $V_{m-1}$ of class $C^{3}$ defined on some region of $R$ about $x^{i}{ }_{0}$ which is imbedded in $\bar{R}_{m}$ by equations of the form $y^{\alpha}=y^{\alpha}\left(x^{i}\right)$ and has the respective forms (29.12) as its successive conformal fundamental forms. For $x=x_{0}{ }_{0}, V_{m-1}$ passes through $P$ so that its conformal tangent vector bein and normal vector take the position $G^{\alpha}{ }_{i 0}, Z^{\alpha}{ }_{0}$ and the values of its mean curvature, gauge function and of the derivative of the latter with respect to $x^{i}$ at $\mathcal{P}$ are $\mu_{0}, \phi_{0}$ and. $\phi_{i 0}$ respectively. Any other hypersurface with these properties will coincide with the $V_{m-1}$ defined above in the common region of definition.

Finally we note that, in accordance with Theorem 28.2, the conformal fundamental tensors determine the $B_{\left(i_{w}\right) \mid\left(l_{w}\right)}$ and the $U$ 's. As a corollary of Theorem 28.2 and Theorem 29.1, we have the second form of the existence theorem:

THEOREM 29.3 Suppose that

$$
\begin{aligned}
& G_{i_{1} l_{1}} d x^{i_{1}} d x^{l_{1}}, G_{\left(i_{2}\right)\left(l_{2}\right)} d x^{i_{1}} d x^{i_{2}} d x^{l_{1}} d x^{l_{2}}, \cdots, \\
& G_{\left(i_{M}\right)\left(l_{M}\right)} d x^{i_{1}} \cdots d x^{i_{M}} d x^{l_{1}} \cdots d x^{l_{M}}, E_{i l} d x^{i} d x^{l}
\end{aligned}
$$

are any symmetric differential forms defined on $R$ whose coefficients satisfy equations (19.12) for suitable functions (29.1). Suppose further that the entire hypothesis of Theorem 29.1 is satisfied with reference to these functions (29.1). Then there exists an $n$-dimensional Riemann space $V_{n}$ of class $C^{3}$ defined on some region of $R$ about $x_{0}{ }_{0}$ which is imbedded in the $\bar{R}_{m}$ of class $C^{4}$ by equations of the form (29.3) and which has the respective differential forms (29.13) as its successive conformal fundamental forms. The conclusion of Theorem 29.1 concerning the initial position and unique determination of $V_{n}$ is also valid here. 
30. Groups of conformal transformations in euclidean space $R_{m}$ and in a conformally euclidean space $\bar{R}_{m}$. If an $S_{m}$ of constant curvature $k$ is mapped on an $S^{\prime}{ }_{m}$ of constant curvature $k^{\prime}$ where $S_{m}$ and $S^{\prime}{ }_{m}$ are spaces of class $C^{3}$ and $m>2$, then one readily finds as a result of (11.3) that $\sigma\left(y^{\alpha}\right)$ satisfies the partial differential equations

$$
\sigma_{\alpha \beta}=-\left\lfloor e^{2 \sigma} k^{\prime}-k+\Delta_{1} \sigma\right] a_{\alpha \beta} / 2 .
$$

Conversely, if a transformation whose mapping function $\sigma\left(y^{\alpha}\right)$ is a solution of (30.1) is applied to $S_{m}$, the image space must be $S_{m}^{\prime}$.

Now suppose that both $S_{m}$ and $S^{\prime}{ }_{m}$ are euclidean spaces. Then $k=k^{\prime}=0$. In this case, (30.1) may be written as

$$
\left(e^{-\sigma}\right)_{\alpha \beta}=\left(\Delta_{1}\left(e^{-\sigma}\right) / 2\left(e^{-\sigma}\right)\right) \cdot a_{\alpha \beta} .
$$

If we let $y^{\alpha}$ be cartesian rectangular coordinates, $a_{\alpha \beta}=\delta^{\alpha}{ }_{\beta}$ and

$$
\left\{\begin{array}{c}
\alpha \\
\beta \gamma
\end{array}\right\}=0
$$

The solution of equations (30.2) in these coordinates is easily found to be

$$
e^{-\sigma}=a,
$$

or

$$
e^{-\sigma}=\sum_{\alpha} b\left(y^{\alpha}-d^{\alpha}\right)^{2},
$$$$
b>0
$$

where $a, b, d^{\alpha}$ are real constants. The point defined by $y^{\alpha}=d^{\alpha}$ is a singular point of any conformal transformation associated with (30.4). We state the above result in the following theorem.

THEOREM 30.1. Every mapping function $\sigma\left(y^{\alpha}\right)$ that maps $R_{m}(m>2)$ on itself conformally must satisfy (30.3) or (30.4), and conversely.

The totality of conformal transformations of $R_{m}$ on itself forms a group $G$. As a consequence of Theorem 30.1, it is easy to establish the theorem of Liouville $\left({ }^{58}\right)$. This theorem states that the most general conformal map of $R_{m}$ $(m>2)$ on itself is the product of an inversion with respect to a hypersphere by a motion ( $\left.{ }^{59}\right)$ or the product of a magnification of similitude by a motion. Now every magnification of similitude is the prcduct of two inversions with respect to hyperspheres having the same center. Hence the group $G$ is the inversive group of transformations of $R_{m}$.

(38) For $m>3$, this theorem was proved by S. Lie [9, p. 186]. Also see [1, pp. 375-376], $[13$, pp. 205-210].

(59) By a motion, we mean any point transformation of $R_{m}$ which is expressed (in cartesian coordinates) by equations having an orthogonal matrix. The Jacobian of the transformation may be either +1 or -1 so that every motion is the product of a finite number of translations, rotations and reflections with respect to hyperplanes. 
It is shown later that the set of geometric objects: $y^{\alpha}, G^{\alpha}{ }_{\left(i_{w}\right)}$ $(w=1,2, \cdots, M), \mu_{\alpha}, \phi, \phi_{i}$ determine the parameters of $G$. For this reason, we call this set of objects the group parameter set or parameter set. We denote this set by the symbol $\Psi$. The order of $\Psi$ is the integer $N_{12} \ldots M$ which is the dimension number of the largest osculating vector space $I_{12} \ldots M$ of $V_{n}$. Of course, as stated in (18.9), the maximum value of the order of $\Psi$ is $m$. It is possible to define the objects belonging to $\Psi$ independently of $V_{n}$ and in any $V_{m}$. The $G^{\alpha}{ }_{\left(i_{w}\right)}$ and $\mu_{\alpha}$ are any set of beins spanning vector spaces and a space vector which obey equations (29.7) where the $B_{\left(i_{w}\right) \mid\left(l_{w}\right)}$ are any surface tensors, $\psi=-\phi$ and $\mu^{\alpha}=a^{\alpha \beta} \mu_{\beta}$. The quantity $\phi$ is any surface scalar and $\phi_{i}$ is $\partial \phi / \partial x^{i}$. Under any conformal transformation of $V_{m}$, the respective objects of $\Psi$ transform according to the laws (2.5),

$$
\bar{G}^{\alpha}{ }_{\left(i_{w}\right)}=G^{\alpha}{ }_{\left(i_{w}\right)},
$$

(6.8), (8.7) and

$$
\bar{\phi}_{i}=\phi_{i}-\sigma_{, i}
$$

respectively.

Since the $G^{\alpha}{ }_{\left(i_{w}\right)}$ are unchanged by a conformal transformation of $V_{m}$, it follows from the laws of change for $a_{\alpha \beta}$ and $\phi$ and from (29.7) that the $B_{\left(i_{w}\right) \mid\left(l_{w}\right)}$ are also unchanged. We also define $\eta_{\alpha}$ corresponding to $\Psi$ by means of (8.10) where $\phi_{\alpha}$ is defined by equations (8.8) written in the form

$$
\phi_{\alpha}=e^{2 \phi} G^{i j} G^{\beta}{ }_{j} a_{\alpha \beta} \phi_{i}
$$

where $G^{i j}$ are the contravariant components of the positive definite tensor $B_{i_{1 \mid l} l_{1}}$. It follows readily from the above laws of change that $\eta_{\alpha}$ transforms according to (8.9) under a conformal transformation of $V_{m}$. If the geometric objects of a parameter set $\Psi$ have the geometric significance described in Theorem 29.1 for some subspace $V_{n}$, then $\Psi$ is said to be associated with $V_{n}$ at the point whose coordinates belong to $\Psi$. If, for two parameter sets $\Psi$ and $\bar{\Psi}$, the tensors $B_{\left(i_{w}\right) \mid\left(l_{w}\right)}$ and $\bar{B}_{\left(i_{w}\right) \mid\left(l_{w}\right)}$ which occur in the conditions (29.7) for the $G^{\alpha}{ }_{\left(i_{w}\right)}$ are equivalent (equal after suitable changes of the $x^{i}$ ), then we say that $\Psi$ and $\bar{\Psi}$ are equivalent. Of course, equivalent parameter sets have the same order. A justification for this definition of equivalent parameter sets lies in the following theorem:

THEOREM 30.2. A conformal transformation of $R_{m}(m>2)$ into itself exists which transforms any given parameter set $\Psi$ into any other equivalent parameter set $\bar{\Psi}$.

The proof of the theorem follows. Let the parameter sets $\Psi$ and $\bar{\Psi}$ be $\left\{y^{\alpha}, G^{\alpha}{ }_{\left(i_{w}\right)}, \mu_{\alpha}, \phi, \phi_{i}\right\}$ and $\left\{\bar{y}^{\alpha}, \bar{G}^{\alpha}{ }_{\left(i_{w}\right)}, \bar{\mu}_{\alpha}, \bar{\phi}, \bar{\phi}_{i}\right\}$ respectively. According to (8.7), if a mapping function $\sigma$ is applied to $\Psi$, the quantity $\phi$ transforms into 
$\phi^{\prime}$ where $\phi^{\prime}=\phi-\sigma$. Hence by means of a conformal transformation( $\left.{ }^{60}\right) \mathcal{T}_{1}$ of $R_{m}$, it is possible to transform $\Psi$ into $\Psi^{\prime}:\left\{y^{\prime \alpha}, G^{\prime \alpha}{ }_{\left(i_{w}\right)}, \mu^{\prime}{ }_{\alpha}, \Phi, \Phi_{i}\right\}$ so that the quantities $\phi^{\prime}, \phi_{i}^{\prime}$ in $\Psi^{\prime}$ corresponding to $\phi, \phi_{i}$ are $\phi, \phi_{i}$. The tensors $B_{\left(i_{w}\right) \mid\left(l_{w}\right)}$ are unchanged by this transformation. Also, by hypothesis, we may consider $B_{\left(i_{w}\right) \mid\left(l_{w}\right)}$ and $\bar{B}_{\left(i_{w}\right) \mid\left(l_{w}\right)}$ as equal after a suitable change in the surface parameters $x^{i}$. Then it follows since $\phi^{\prime}=\Phi$ that the conditions $(29.7)$ on the $G^{\prime{ }^{\prime}{ }_{\left(i_{w}\right)}}$ and $\bar{G}^{\alpha}{ }_{\left(i_{w}\right)}$ are identical; that is, the vector spaces $G^{\prime{ }^{\alpha}}{ }_{\left(i_{w}\right)}$ and $\bar{G}^{\alpha}{ }_{\left(i_{w}\right)}$ have the same inner orientation. It follows that a motion $\mathcal{T}_{2}$ in $R_{m}$ exists so that $y^{\prime \alpha}=\bar{y}^{\alpha}, G^{\prime \alpha}{ }_{\left(i_{w}\right)}=\bar{G}^{\alpha}{ }_{\left(i_{w}\right)}$. Under this motion, the parameter set $\Psi^{\prime}$ becomes the parameter set $\Psi^{\prime \prime}:\left\{\bar{y}^{\alpha}, \bar{G}^{\alpha}{ }_{\left(i_{w}\right)}, \mu^{\prime \prime}{ }_{\alpha}, \phi^{\prime \prime}, \phi^{\prime \prime}{ }_{i}\right\}$. We write $\eta^{\prime \prime}{ }_{\alpha}$ and $\bar{\eta}_{\alpha}$ for the quantities corresponding to $\Psi^{\prime \prime}$ and $\bar{\Psi}$ and now consider the following two cases :

(1) Suppose that $\eta^{\prime \prime}{ }_{\alpha}=\bar{\eta}_{\alpha}$. This is equivalent to $\mu^{\prime \prime}{ }_{\alpha}=\bar{\mu}_{\alpha}$ and $\phi^{\prime \prime}{ }_{i}=\bar{\phi}_{i}$. Then a unique positive constant $a$ exists so that $e^{\bar{\phi}}=a e^{\phi^{\prime \prime}}$. If we subject $R_{m}$ to the magnification of similitude $\mathcal{G}_{3}$ determined by $e^{-\sigma}=a$, it follows from (6.8), (8.7) and (30.6) that $\left\{\mu^{\prime \prime}{ }_{\alpha}, \phi^{\prime \prime}, \phi^{\prime \prime}{ }_{i}\right\}$ transforms into $\left\{\bar{\mu}_{\alpha}, \bar{\phi}, \phi_{i}\right\}$. According to (2.5) and (30.5), $\bar{y}^{\alpha}, \bar{G}^{\alpha}{ }_{\left({ }_{i w}\right)}$ of $\Psi^{\prime \prime}$ remain unchanged. Consequently the conformal transformation $\mathcal{T}_{3} \mathcal{V}_{2} \mathcal{V}_{1}$ changes $\Psi$ into $\bar{\Psi}$.

(2) Suppose at least one of the equations in the hypothesis of case (1) is untrue. We choose cartesian rectangular coordinates in $R_{m}$ so that the $\bar{y}^{\alpha}=0$ (or transform the point whose coordinates belong to $\Psi^{\prime \prime}$ and $\bar{\Psi}$ to the origin by a motion). We now determine a mapping function $\sigma$ satisfying (30.4) which transforms $\Psi^{\prime \prime}$ into $\bar{\Psi}$. At the origin, we find from (30.4) that

$$
e^{-\sigma}=b \sum_{\beta} d^{\beta^{2}}, \quad \sigma_{, \alpha}=2 d^{\alpha} / \sum_{\beta} d^{\beta^{2}} .
$$

If we substitute these values in (8.7) and (8.9), we find that

$$
e^{\bar{\phi}-\phi^{\prime \prime}}=b \sum_{\beta} d^{\beta^{2}}, \quad \eta_{\alpha}^{\prime \prime}-\bar{\eta}_{\alpha}=2 d^{\alpha} / \sum_{\beta} d^{\beta^{2}} .
$$

Since $\eta^{\prime \prime}{ }_{\alpha} \neq \eta_{\alpha}$, at least one $d^{\alpha}$ differs from zero. Hence, from the second set of equations (30.7),

$$
\frac{1}{4} \sum_{\alpha}\left(\eta^{\prime \prime}{ }_{\alpha}-\bar{\eta}_{\alpha}\right)^{2}=1 / \sum_{\beta} d^{\beta^{2}}
$$

Therefore the solution of $(30.7)$ is

$$
\begin{aligned}
b & =\frac{1}{4} e^{\bar{\phi}-\phi^{\prime \prime}} \cdot \sum_{\beta}\left(\eta^{\prime \prime}{ }_{\beta}-\bar{\eta}_{\beta}\right)^{2}, \\
d^{\alpha} & =2\left(\eta^{\prime \prime}{ }_{\alpha}-\bar{\eta}_{\alpha}\right) / \sum_{\beta}\left(\eta^{\prime \prime}{ }_{\beta}-\bar{\eta}_{\beta}\right)^{2} .
\end{aligned}
$$

It is immediate that $b$ is positive so that values $b, d^{\alpha}$ given by (30.8) may be

${ }^{\left({ }^{60}\right)}$ The existence of conformal transformations of $R_{m}$ with this property follows from the discussion which appears below. 
used to define a mapping function $\sigma$ in accordance with (30.4). Since not all the $d^{\alpha}$ are zero, the origin is not a singular point of any transformation associated with (30.4). Any such transformation $\mathcal{G}_{3}$ would transform $\left\{\mu^{\prime \prime}{ }_{\alpha}, \phi^{\prime \prime}, \phi^{\prime \prime}{ }_{i}\right\}$ into $\left\{\bar{\mu}_{\alpha}, \bar{\phi}, \bar{\phi}_{i}\right\}$. Since $\bar{y}^{\alpha}, \bar{G}^{\alpha}{ }_{\left(i_{w}\right)}$ remain unchanged by $\mathcal{T}_{3}$, the conformal transformation $\mathcal{V}_{3} \mathcal{V}_{2} \mathcal{V}_{1}$ changes $\Psi$ into $\bar{\Psi}$. This completes the proof of the theorem.

Now suppose that the geometric objects in $\bar{\Psi}$ are fixed while those which belong to $\Psi$ range over all admissible values. Then the corresponding conformal transformations range over the totality of transformations belonging to the inversive group $G$. Hence the geometric objects of $\Psi$ determine the parameters of $G$. As noted in a previous paper $\left({ }^{61}\right)$, a count of the independent parameters proves that $G$ has exactly $(m+1)(m+2) / 2$ essential parameters.

Let $\bar{R}_{m}(m>2)$ be a conformally euclidean space and suppose $\mathcal{T}_{1}$ is a conformal transformation which transforms $\bar{R}_{m}$ into $R_{m}$. If $\mathcal{T}$ is any transformation belonging to $G$, then the conformal transformation $\mathcal{V}_{1}^{-1} \mathcal{G}_{1}$ maps $\bar{R}_{m}$ on itself. The totality of these transformations $\{\overline{\mathcal{T}}\}$ forms the complete group $\bar{G}$ of conformal transformations of $\bar{R}_{m}$ upon itself. The group $\bar{G}$ is the conformal image in $\bar{R}_{m}$ of the group $G$ in $R_{m}$ and is independent of the particular mapping $\mathcal{T}_{1}$. It is clear that Theorem 30.2 may be proved to be valid for parameter sets in $\bar{R}_{m}$ by means of this correspondence between $G$ and $\bar{G}$. Another consequence is that every conformally euclidean space $\bar{R}_{m}(m>2)$ admits a continuous group of conformal transformations on itself having $(m+1)(m+2) / 2$ essential parameters.

31. The conformal equivalence theorem. The fundamental theorem of Riemannian geometry for a subspace in a euclidean space $R_{m}$ or a space of constant curvature $S_{m}$ is the congruence theorem. We are now in a position to establish easily the analogue of this theorem for conformal differential geometry.

TheOREM('62) 31.1. Let (1) $\bar{R}_{m}$ and (2) $\bar{R}_{m}$ be conformally euclidean spaces of class $C^{4}$ and dimensionality $m>2$ and let (1) $V_{n}$ and ${ }_{(2)} V_{n}$ be subspaces of class $C^{3}$ in (1) $\bar{R}_{m}$ and (2) $\bar{R}_{m}$ respectively of dimensionality $n>1$. Suppose that the fundamental equations of (1) $V_{n}$ and (2) $V_{n}$ are valid and suppose that the corresponding conformal fundamental forms of (1) $V_{n}$ and (2) $V_{n}$ are equal. Then a conformal transformation exists so that (1) $\bar{R}_{m} \rightleftarrows(2) \bar{R}_{m}$ and (1) $V_{n} \rightleftarrows(2) V_{n}$.

In short, this theorem states that $n$-dimensional subspaces of $\bar{R}_{m}$ 's whose corresponding conformal fundamental forms are equal are conformally equivalent. To prove the theorem, we note that conformal transformations $\mathcal{T}_{1}$ and $\mathfrak{T}_{2}$ exist which map (1) $\bar{R}_{m}$ and (2) $\bar{R}_{m}$ respectively on $R_{m}$. For these transformations we also have (1) $V_{n} \rightarrow_{(1)} \bar{V}_{n}$ and (2) $V_{n} \rightarrow_{(2)} \bar{V}_{n}$ respectively, where (1) $\bar{V}_{n}$

(61) The details of the present discussion appear in [8, pp. 472-473].

${ }^{(62)}$ Various modifications of this theorem appear in the remarks at the conclusion of the proof. 
and (2) $\bar{V}_{n}$ are subspaces in $R_{m}$. Since the conformal fundamental forms exist for (1) $V_{n}$ and (2) $V_{n}$, in accordance with Theorem 19.1, they also exist for (1) $\bar{V}_{n}$ and (2) $\bar{V}_{n}$ and corresponding forms of (1) $\bar{V}_{n}$ and (2) $\bar{V}_{n}$ are equal. Furthermore, as a consequence of the remarks in the summary of chapter $\mathrm{V}$, the fundamental equations of (1) $\bar{V}_{n}$ and (2) $\bar{V}_{n}$ are valid.

Let $\Phi_{1}$ and $\Phi_{2}$ be points which belong to (1) $\bar{V}_{n}$ and (2) $\bar{V}_{n}$ respectively which have the same parameters $x^{i}{ }_{0}$ and such that the corresponding conformal fundamental forms, evaluated at $P_{1}$ and $P_{2}$, are equal. Let $\Psi_{1}$ and $\Psi_{2}$ be the group parameter sets associated with (1) $\bar{V}_{n}$ and (2) $\bar{V}_{n}$ respectively at $P_{1}$ and $P_{2}$. As a consequence of Theorem 30.2, a conformal transformation $\tau$ of $R_{m}$ into itself exists which transforms $\Psi_{1}$ into $\Psi_{2}$. This same transformation transforms (1) $\bar{V}_{n}$ into some subspace $V^{\prime}{ }_{n}$ passing through $\Phi_{2}$ for $x^{i}=x^{i}{ }_{0}$ and having the associated parameter set $\Psi_{2}$ at $P_{2}$. For reasons like those given in connection with (1) $\bar{V}_{n}$ and (2) $\bar{V}_{n}$, the fundamental forms for $V^{\prime}{ }_{n}$ exist and are equal to the corresponding forms of ${ }_{(2)} \bar{V}_{n}$. Also, the fundamental equations of $V^{\prime}{ }_{n}$ are valid. It follows from Theorem 29.3 that $V^{\prime}{ }_{n}$ coincides with (2) $\bar{V}_{n}$ in a sufficiently small neighborhood of $\mathcal{P}_{2}$. Hence the transformation $\mathcal{T}^{-1}{ }_{2} \mathcal{T}_{\mathcal{V}_{1}}$ transforms (1) $\bar{R}_{m}$ into (2) $\bar{R}_{m}$ mapping sufficiently small regions of (1) $V_{n}$ and (2) $V_{n}$ on each other. This proves Theorem 31.1.

Remark 1. Suppose that the enveloping space is an $R_{m}$ or an $S_{m}$ and we limit the conformal transformations to mappings between these spaces of constant curvature only. For reasons like those stated in Remark 3 of $\$ 29$, the hypothesis of Theorem 31.1 may be weakened so that $R_{m}$ or $S_{m}$ is of class $C^{3}$ instead of class $C^{4}$ and the remainder of the theorem will hold as stated.

Remark 2. An analogous equivalence theorem exists in which the conformal measure tensors and deviation tensor occupy roles similar to those of the coefficients of the conformal fundamental forms in Theorem 31.1. The proof of this theorem is based upon Theorem 29.1 instead of Theorem 29.3.

Remark 3. If $m=n+1$, it follows from (20.11) that the condition ${ }_{(1)} B_{\left(i_{2}\right) \mid\left(l_{2}\right)}={ }_{(2)} B_{\left(i_{2}\right) \mid\left(l_{2}\right)}$ in the conformal equivalence theorem may be replaced by ${ }_{(1)} B_{i j}= \pm{ }_{(2)} B_{i j}$.

As a result of the existence and equivalence theorems, it is clear that equations which assign arbitrary values to the coefficients of the conformal fundamental forms of $V_{n}$ (subject, of course, to (19.12) and the fundamental equations) may be regarded as conformal intrinsic equations of $V_{n}$ in $\bar{R}_{m}$. These equations determine the $V_{n}$ up to a conformal transformation of the space. A detailed conformal differential geometry of subspaces based upon these fundamental forms may be developed.

32. Conformal differential forms. A conformal differential form

$$
T_{\left(i_{v}\right)} d x^{i_{1}} d x^{i_{2}} \ldots d x^{i_{v}}
$$

of a subspace $V_{n}$ in $V_{m}$ whose equations are $y^{\alpha}=y^{\alpha}\left(x^{i}\right)$ is a differential form whose coefficients $T_{\left(i_{v}\right)}$ are functions of the variables 


$$
\begin{gathered}
y^{\alpha}\left(x^{i}\right), \partial y^{\alpha} / \partial x^{i}, \partial^{2} y^{\alpha} / \partial x^{i} \partial x^{i}, \cdots, a_{\alpha \beta}\left[y^{\gamma}\left(x^{i}\right)\right], \\
\partial a_{\alpha \beta} / \partial y^{\gamma}, \partial^{2} a_{\alpha \beta} / \partial y^{\gamma} \partial y^{\delta}, \ldots
\end{gathered}
$$

defined along $V_{n}$ and which, at any point $\mathcal{P}$ of $V_{n}$, has the same value for any admissible change of coordinates $y^{\alpha}$ or of the parameters $x^{i}$ and whose value also remains unchanged if $V_{m}$ is mapped conformally on $\bar{V}_{m}$. This last condition, which gives (32.1) its conformal character, is equivalent to the assumption that (32.1) is invariant if the $a_{\alpha \beta}$ and their derivatives are replaced respectively by $e^{2 \sigma} a_{\alpha \beta}$ and their derivatives while the other variables in (32.2) are unchanged.

The simplest conformal differential forms of $V_{n}$ are its conformal fundamental forms. The most important problem concerning conformal forms is the discovery of all such differential forms. It is seen from the definition that this question is equivalent to finding all conformal tensors $T_{\left(i_{v}\right)}$ which depend upon the variables (32.2). In the sequel, we solve this problem when $V_{n}$ is a subspace of any conformally euclidean space $\bar{R}_{m}$. We prove the following theorem of which the converse statement has already been demonstrated.

THEOREM 32.1. The most general conformal differential form of a subspace $V_{n}(n>1)$ in an $\bar{R}_{m}$ is a differential form whose coefficients are tensor functions of the conformal fundamental tensors and conformal Riemann tensor of $V_{n}$ and of their conformal covariant derivatives. Conversely, every such differential form is a conformal differential form.

The proof follows. We consider a conformal differential form (32.1) defined at any point of an $n$-dimensional subspace in an $\bar{R}_{m}$. The coefficients $T_{\left(i_{v}\right)}$ are conformal tensors which depend upon the variables (32.2). By means of a conformal mapping of $\bar{R}_{m}$ on $R_{m}$, (32.1) becomes a conformal differential form defined at any point $P$ of a suisspace $V_{n}$ in $R_{m}$. We therefore first discuss conformal forms of $V_{n}$ in $R_{m}$.

By virtue of (29.4), the successive partial derivatives of $y^{\alpha}$ with respect to the $x^{i}$ may be written as functions of $U^{\left(h_{w}\right)}{ }_{\left(i_{w+1}\right)}, U^{\left(h_{w-1}\right)}{ }_{\left(i_{w+1}\right)}, G_{i j}, E_{i j}, K_{i j}$, $\Gamma_{i j}^{h}$ and their derivatives with respect to $x^{k}$ and of $a_{\alpha \beta}$ and their derivatives with respect to $y^{\gamma}$ and of $y^{\alpha}, G^{\alpha}{ }_{\left(i_{w}\right)}, \psi, \psi_{i}, \mu^{\alpha}$. (Here $w$ has the range $1,2, \cdots, M$.) According to Theorem 28.2, $U^{\left(h_{w}\right)}{ }_{\left(i_{w+1}\right)}, U^{\left(h_{w-1}{ }_{\left(i_{w+1}\right)}\right.}$ may be replaced by a combination of the conformal fundamental tensors (except for unessential additive null tensors).

Hence $T_{\left(i_{v}\right)}$ is a tensor function which depends upon the variables

$$
y^{\alpha}, G^{\alpha}{ }_{\left(i_{w}\right)}, \psi, \psi_{i}, \mu^{\alpha}, a_{\alpha \beta}, \frac{\partial a_{\alpha \beta}}{\partial y^{\gamma}}, \frac{\partial^{2} a_{\alpha \beta}}{\partial y^{\gamma} \partial y^{\delta}}, \cdots, \cdots,
$$

$$
G_{i j}, \Gamma^{h_{i j}}, \frac{\partial}{\partial x^{k}} \Gamma^{h_{i j}}, \cdots, G_{\left(i_{2}\right)\left(i_{2}\right)}, G_{\left(i_{2}\right)\left(j_{2}\right): k}, G_{\left(i_{2}\right)\left(i_{2}\right): k l}, \cdots,
$$$$
G_{\left(i_{M}\right)\left(i_{M}\right)}, G_{\left(i_{M}\right)\left(i_{M}\right): k}, G_{\left(i_{M}\right)\left(i_{M}\right): k l}, \cdots, E_{i j}, E_{i j: k}, E_{i j: k l}, \cdots
$$ 
Of these variables, only the conformal fundamental tensors and their conformal derivatives, $\Gamma^{h_{i j}}$ and its partial derivatives and the $G^{\alpha}{ }_{\left(i_{w}\right)}$, have a conformal character. When the enveloping $R_{m}$ is subjected to a conformal transformation, $\bar{y}^{\alpha}=y^{\alpha}, \bar{x}^{i}=x^{i}, \bar{T}_{\left(i_{v}\right)}$ is the same function with the variables (32.3) replaced by the variables $\bar{y}^{\alpha}, G^{\alpha}{ }_{\left(i_{w}\right)}, \Psi, \bar{\psi}_{i}$, and so on, which correspond to (32.3) under the conformal mapping and $\bar{T}_{\left(i_{v}\right)}=T_{\left(i_{v}\right)}$ at corresponding points.

Of the variables (32.3), it is very plausible that the effective presence (other than in conformal derivatives) of $\Gamma^{h_{i j}}$ and its successive derivatives in $T_{\left(i_{v}\right)}$ must be limited to combinations which are functions of the conformal Riemann curvature tensor $K^{h}{ }_{i j k}$ of $V_{n}$ and its successive conformal derivatives. To prove this conjecture, we choose a system of conformal normal parameters $\left\{x^{i}\right\}$ whose center is an arbitrary point $P$ of $V_{n}$. Then, in a neighborhood about $\left({ }^{63}\right) \mathcal{P}$,

$$
\Gamma^{h}{ }_{i j} x^{i} x^{j}=0 .
$$

Differentiation of (32.4) with respect to $x^{k}$, followed by multiplication by $x^{k}$ and simplification by means of (32.4), leads to the equations

$$
\left(\partial \Gamma_{i j}^{h} / \partial x^{k}\right) x^{i} x^{j} x^{k}=0 .
$$

Analogous equations for the higher derivatives of $\Gamma^{h}{ }_{i j}$ may be obtained from (32.5) in a similar way. Successive differentiation of (32.4), (32.5), and so on, and evaluation at $\mathcal{P}$ leads to the following equations which are valid at $P$ :

$$
\Gamma_{i j}^{h}=0, \quad \sum \partial \Gamma^{h}{ }_{i j} / \partial x^{k}=0, \quad \sum \partial^{2} \Gamma^{h}{ }_{i j} / \partial x^{l} \partial x^{k}=0, \cdots .
$$

In these equations the summation symbol indicates the symmetric sum over all indices except $h$.

It follows from (14.1) and the first two equations of (32.6) that, at $P$,

$$
\partial \Gamma^{h}{ }_{i j} / \partial x^{k}=\left[K^{h}{ }_{i k j}+K^{h}{ }_{j k i}\right] / 3 .
$$

In a similar way, by successive differentiation of (14.1) and use of the equations of (32.6), we may express the values of the higher partial derivatives of $\Gamma_{i j}{ }_{i j}$ at $P$ in terms of $K^{h_{i j k}}$ and its various conformal derivatives. Hence the quantities

$$
\Gamma_{i j}^{h}, \partial \Gamma_{i j}^{h} / \partial x^{k}, \cdots
$$

may be replaced by

$$
0,\left[K^{h}{ }_{i k j}+K^{h}{ }_{j k i}\right] / 3, \cdots
$$

at $P$ when the parameter system $\left\{x_{i}\right\}$ is conformal normal. Now $T_{\left(i_{v}\right)}$ as well as the quantities (32.8) and all the variables of (32.3) except (32.7) are tensors. Therefore if the variables $(32.7)$ in $T_{\left(i_{v}\right)}$ are replaced by $(32.8)$ at $\mathcal{P}$,

(63) For a proof of this characteristic property of normal coordinates, see $[4$, p. 54]. 
$T_{\left(i_{v}\right)}$ becomes a tensor function of tensor variables and hence is valid under all parameter transformations. Since $P$ is any point of $V_{n}$, this remark shows that the variables (32.7) may be replaced by combinations of $K^{h}{ }_{i j k}$ and its successive conformal derivatives $\left({ }^{64}\right)$.

We now show that $y^{\alpha}, G^{\alpha}{ }_{\left(i_{w}\right)}, \psi, \psi_{i}, \mu^{\alpha}, a_{\alpha \beta}, \partial a_{\alpha \beta} / \partial y^{\gamma}, \partial^{2} a_{\alpha \beta} / \partial y^{\delta} \partial y^{\gamma}, \ldots$ cannot be present in $T_{\left(i_{v}\right)}$ as effective independent variables. In $R_{m}$, the coordinates $y^{\alpha}$ may be chosen so that they belong to a rectangular cartesian coordinate system $\mathscr{W}$ such that $a_{\alpha \beta}=\delta^{\alpha}{ }_{\beta}, \partial a_{\alpha \beta} / \partial y^{\gamma}=0, \partial^{2} a_{\alpha \beta} / \partial y^{\delta} \partial y^{\gamma}=0, \ldots$ throughout a region of $R_{m}$ containing any point $\mathcal{P}$ of $V_{n}$ and at $\mathcal{P}, y^{\alpha}=0$, $G^{\alpha}{ }_{\left(i_{w}\right)}=G^{\alpha}{ }_{\left(i_{w}\right) 0}$. Here the $G^{\alpha}{ }_{\left(i_{w}\right) 0}$ are any fixed set of numbers which satisfy the $B$-conditions (29.7). If $R_{m}$ is subjected to a conformal transformation( ${ }^{65}$ )

$$
y^{\alpha}=y^{\alpha}\left(\bar{y}^{1}, \bar{y}^{2}, \cdots, \bar{y}^{m}\right), \quad \bar{y}^{\alpha}=\bar{y}^{\alpha}\left(y^{1}, y^{2}, \cdots, y^{m}\right)
$$

belonging to $G, V_{n}$ is mapped on another subspace $\bar{V}_{n}$ in $R_{m}$ and $P$ corresponds to $\bar{P}$. In this transformation, the $\bar{y}^{\alpha}$ have been chosen so that they belong to a rectangular cartesian coordinate system $\overline{\mathscr{W}}$ such that $\bar{a}_{\alpha \beta}=\delta^{\alpha}{ }_{\beta}$, $\partial \bar{a}_{\alpha \beta} / \partial \bar{y}^{\gamma}=0, \partial^{2} \bar{a}_{\alpha \beta} / \partial \bar{y}^{\delta} \partial y^{\gamma} \stackrel{\perp}{0}, \cdots$ throughout a region of $R_{m}$ containing $\bar{P}$ and at $\bar{P}, \bar{y}^{\alpha}=0, \bar{G}^{\alpha}{ }_{\left(i_{w}\right)}=G^{\alpha}{ }_{\left({ }_{i w}\right) 0}$. It is clear that in the coordinate systems $\mathscr{W}$ and $\overline{\mathscr{W}}$, the values of the $a_{\alpha \beta}$ and their derivatives as well as of $G^{\alpha}{ }_{\left(i_{\omega}\right)}$ at $\mathscr{P}$ remain unchanged by conformal transformations of $G$. Of course, the conformal Riemann tensor and the conformal fundamental tensors of $V_{n}$ and their conformal derivatives also remain constant. Hence, in these coordinate systems, the only possible variables of (32.1) are $\psi, \psi_{i}, \mu^{\alpha}$.

As a result of Theorem 30.2, a conformal transformation of $G$ exists which leaves the values at $P$ of $y^{\alpha}, G^{\alpha}{ }_{\left({ }_{i w}\right)}$ unaltered and which transforms the set $\psi, \psi_{i}, \mu^{\alpha}$ at $\Phi$ into any other set $\psi, \psi_{i}, \bar{\mu}^{\alpha}$ [subject to the last equations of (29.7)]. Hence $\psi, \psi_{i}, \mu^{\alpha}$ behave like independent variables with respect to conformal transformations of $G$. It follows that if any of $\psi, \psi_{i}, \mu^{\alpha}$ are effective variables, (32.1) cannot remain invariant at $P$. This contradiction shows that (32.1) is independent of $\psi, \psi_{i}, \mu^{\alpha}$ at each point of $V_{n}$. Hence in the coordinate systems $\mathscr{W}, \overline{\mathscr{W}}, T_{\left(i_{v}\right)}$ is a tensor function of the conformal Riemann tensor and conformal fundamental tensors of $V_{n}$ as well as their various conformal derivatives. Hence $T_{\left(i_{v}\right)}$ may be written as

$$
\begin{aligned}
T_{\left(i_{v}\right)}= & T\left[G_{i j}, K^{h_{i j k}}, K^{h_{i j k: l}}, \cdots, E_{i j}, E_{i j: k}, E_{i j: k l}, \cdots,\right. \\
& G_{\left(i_{2}\right)\left(i_{2}\right)}, G_{\left(i_{2}\right)\left(i_{2}\right): k}, G_{\left(i_{2}\right)\left(i_{2}\right): k l}, \cdots, \cdots, G_{\left(i_{M}\right)\left(j_{M}\right)}, \\
& \left.G_{\left(i_{M}\right)\left(j_{M}\right): k}, G_{\left(i_{M}\right)\left(i_{M}\right): k l}, \cdots\right]
\end{aligned}
$$

in the coordinate systems $\mathscr{W}, \overline{\mathscr{W}}$.

Since (32.9) is a tensor equation, it follows that (32.9) is valid for any allowable coordinate system in $R_{m}$. This observation proves the theorem for

${ }^{(64)}$ This result also follows from the replacement theorem of Thomas [15, p. 109].

(65) In this proof, we no longer assume that points with the same coordinates correspond as was done previously. 
subspaces in $R_{m}$ and conformal transformations which belong to $G$. But all the tensors of both members of (32.9) are conformal tensors and are unchanged by any conformal mapping of $R_{m}$ on an $\bar{R}_{m}$. By means of this map, $V_{n}$ in $R_{m}$ becomes an $n$-dimensional subspace $\bar{V}_{n}$ in $\bar{R}_{m}$. Also all $n$-dimensional subspaces of $\bar{R}_{m}$ may be obtained as conformal images of $V_{n}$ 's in $R_{m}$. Consequently (32.9) is valid for any subspace of $\bar{R}_{m}$. This remark concludes the proof of Theorem 32.1 .

It is clear that a similar proof in ordinary Riemannian geometry would establish the known theorem that every metric differential form of a subspace $V_{n}(n>1)$ in an $R_{m}$ or $S_{m}$ is a differential form whose coefficients are tensor functions of the (metric) fundamental tensors and Riemann curvature tensor of $V_{n}$ and of their covariant derivatives, and conversely. Finally, we note that Theorem 32.1 is probably false in any enveloping Riemann space $V_{m}$ which is not conformally euclidean. A proof of this statement would probably be analogous to the one for curves which has already been given by the author [8, pp. 477-478] and would be based upon the nonzero conformal tensor $\rho_{\alpha \beta \gamma}$ if $m=3$ or $C^{\alpha}{ }_{\beta \gamma \delta}$ if $m>3$.

\section{EXCEPTIONAL SUBSPACES}

33. Conformal null subspaces. The results of the preceding chapters are meaningless in the case of a subspace $V_{n}$, contained in a general Riemann space $V_{m}$, whose relative conformal curvature $\Lambda$ vanishes identically. In this case the conformal measure tensors $G_{i j}, A_{\alpha \beta}$ are zero tensors, and conversely. This means that the conformal length of any curve on $V_{n}$ or of any surface or space vector defined at any point of $V_{n}$ is zero. For this reason, we call any $V_{n}(n>1)$ with $\Lambda \equiv 0$ which is a subspace of $V_{m}$ a conformal null subspace $\left({ }^{66}\right)$. We impose the condition $n>1$ since, according to Theorem 6.1, $\Lambda \equiv 0$ if $n=1$. The remaining case, $n=1$ (curve), is considered in the next section. We now derive a number of simple properties of these subspaces. The first of these is the theorem:

THEOREM 33.1. The conformal image of a conformal null subspace is a conformal null subspace.

This is an immediate consequence of the definition and (6.13). Also, according to Theorem 6.1, it follows that $V_{n}$ is a conformal null subspace of $V_{m}$ if and only if all its points are umbilical points. As a consequence of this statement, we note that (1) A $V_{m}$ admits conformal null subspaces at each point and for every initial set of values for $G^{\alpha}{ }_{i}, \mu^{\alpha}$ if and only if( $\left.{ }^{67}\right) V_{m}$ is an ${ }^{\left({ }^{68}\right)} \bar{R}_{m}$.

${ }^{\left({ }^{6}\right)}$ In the following discussion, we do not consider the non-real null subspaces which are solutions of $d s^{\prime 2}=a_{\alpha \beta} d y^{\alpha} d y^{\beta}=0$. A prerequisite for the investigation of these subspaces is the extension (if possible) of the region of definition of the metric tensor $a_{\alpha \beta}$ from the $m$-dimensional real number space to the $2 m$-dimensional complex number space.

(67) A proof of this statement may be based upon the discussion in [3, pp. 144-145].

${ }^{(68)}$ On the other hand, conformal null curves exist at each point and for every initial set of values for $G^{\alpha}{ }_{i}, \mu^{\alpha}$ in any enveloping space $V_{m}$. For a proof of this statement, see $[8, \S 10]$. 
(2) In contrast to the case of Riemannian geometry, real null subspaces may exist in $V_{m}$ even when its Riemannian metric is positive definite. Now, from Theorem 9.3, every $V_{n}$ is conformally equivalent to a minimal subspace. Hence if $V_{n}$ is a conformal null subspace, it may be transformed into an umbilical subspace of zero mean curvature. The converse of this statement is immediate. These remarks prove the following theorem.

THEOREM 33.2. A subspace $V_{n}(n>1)$ in $a V_{m}$ is a coiformal null subspace if and only if a Riemann space $\bar{V}_{m}$ and a conformal transformation of $V_{m}$ on $\bar{V}_{m}$ exist such that $V_{n}$ is mapped by this transformation on a totally geodesic subspace of $\bar{V}_{m}$.

It is easy to see that a $V_{n}$ in an $R_{m}$ is umbilical if and only if $V_{n}$ is an $n$-dimensional plane or sphere. It is also clear that a conformal map (belonging to $G$ ) of $R_{m}$ on itself exists so that any $n$-sphere transforms into an $n$-plane. Furthermore, if an $\bar{R}_{m}$ is mapped conformally on $R_{m}$, any conformal null subspace of $\bar{R}_{m}$ is transformed into a conformal null subspace of $R_{m}$ and hence is conformally equivalent to an $n$-plane in $R_{m}$. We state these results in the following theorem.

THEOREM 33.3. Every conformal null subspace $V_{n}$ of $R_{m}$ is an $n$-plane or an $n$-sphere, and conversely. The necessary and sufficient condition that a subspace $V_{n}(n>1)$ in an $\bar{R}_{m}$ be a conformal null subspace is that $V_{n}$ be the conformal image of an n-plane in $R_{m}$. All n-dimensional null subspaces in $\bar{R}_{m}$ 's are conformally equivalent.

34. Conformal curve theory. The conformal theory developed in this paper does not apply to one-dimensional subspaces of $V_{m}$ (curves) since $\Lambda$ is identically zero in this case. The conformal geometry of a curve was obtained in a previous paper by the author [8], using "conformetric" vectors. As was indicated in that paper, the use of conformetric vectors is not essential and the entire theory may be developed in terms of conformal geometric objects. In this section, we show how this may be done, utilizing the methods of the present paper. The method which appears below is contained, in essence, in our previous paper $\left({ }^{69}\right)$ [8].

We consider a curve $V_{1}$ of class $C^{r}$ with $r \geqq 3$ in a Riemann space $V_{m}$. The equations (4.3) of $V_{1}$ may be written as

$$
y^{\alpha}=y^{\alpha}\left(x^{1}\right)
$$

where, according to our assumptions of $\S 4, d y^{\alpha} / d x^{1} \neq 0$. The metric tensor of $V_{1}$ is given by the formula

$$
g_{11}=a_{\alpha \beta} g_{1}^{\alpha} g^{\beta}{ }_{1}
$$

(69) For purposes of comparison, we note that $y^{\alpha}, a_{\alpha \beta},(v) V^{\alpha}, \mu^{\alpha}, \sigma, \alpha, \sigma_{\alpha \beta}, \rho, \rho_{\alpha \beta}, \Omega, v, \zeta^{\alpha}$, (v) $k,(v) Z^{\alpha}, \mathrm{I}_{\beta \gamma,(v)}^{\alpha} J$ of this section appear in [8] as $x^{i}, g_{i j,(\alpha)} V^{i}, \mu^{i}, \sigma, i, \sigma_{i j}, R, R_{i j}, J, K, \eta^{i}, k_{\alpha}$, (a) $\lambda^{i}, \Gamma^{\prime i}{ }_{j k}, J_{\alpha}$ respectively. 
where $g^{\alpha}{ }_{1}=d y^{\alpha} / d x^{1}$. It is convenient to introduce the parameter $s$ by means of the allowable parameter transformation

$$
s=\int\left(g_{11}\right)^{1 / 2} \cdot d x^{1}
$$

The parameter $s$ is an arc length parameter determined up to an additive constant and a choice of sign. For this parameter system, we find that $\left({ }^{70}\right)$

$$
g_{11}=1, \quad\left\{\begin{array}{c}
1 \\
11
\end{array}\right\}=0
$$

so that $g^{\alpha}$ is a unit vector. In this case, we write $g^{\alpha}{ }_{1}$ as ${ }_{(1)} \nu^{\alpha}$. It is the unit tangent vector of $V_{1}$.

According to the definition of the mean curvature normal given by (5.9),

$$
\nu^{\alpha, 8}=\mu^{\alpha}
$$

where the subscript $s$ indicates that the parameter $x^{1}$ is $s$. The vector $\mu^{\alpha}$ is usually called the principal normal of $V_{1}$. We may write $\mu^{\alpha}$ as

$$
\mu^{\alpha}={ }_{(1)} k_{(2)} \nu^{\alpha}
$$

where ${ }_{(1)} k$ is the first curvature of $V_{1}$ and ${ }_{(2)} \nu^{\alpha}$ is the first unit normal of $V_{1}$. Differentiation of ${ }_{(2)} \nu^{\alpha}$ and use of the fact that ${ }_{(1)} \nu^{\alpha},{ }_{(2)} \nu^{\alpha}$ are orthogonal unit vectors leads to the equations

$$
{ }_{(2)} \nu^{\alpha, 8}=-{ }_{(1)} k_{(1)} \nu^{\alpha}+{ }_{(2)} k_{(3)} \nu^{\alpha}
$$

where (2) $k$ is the second curvature of $V_{1}$ and ${ }_{(3)} \nu^{\alpha}$ is the second unit normal of $V_{1}$. Equations (34.2) and (34.4) are the first two sets of the familiar Frenet equations of $V_{1}$. The general equations

$$
{ }_{(v)} \nu^{\alpha}, s=-{ }_{(v-1)} k_{(v-1)} \nu^{\alpha}+{ }_{(v)} k_{(v+1)} \nu^{\alpha}, \quad{ }_{(0)} k={ }_{(m)} k=0, v=1,2, \cdots, m,
$$

may easily be derived by mathematical induction if the successive unit normals are of class $C^{1}$.

The relationship between the principal normals of $V_{1}$ in $V_{m}$ and of its conformal image $\bar{V}_{1}$ in $\bar{V}_{m}$ is given by (6.7). If (6.7) is differentiated covariantly and use is made of

$$
\text { (1) } \bar{\nu}^{\alpha}=e^{-\sigma}(1) \nu^{\alpha}
$$

and of (2.4), (2.6), (6.3), (34.2), (34.3) and (34.4), we find that

$$
\begin{aligned}
\left(d_{(1)} \bar{k} / d \bar{s}\right)_{(2)} \bar{\nu}^{\alpha}+{ }_{(1)} \bar{k}_{(2)} \bar{k}_{(3)} \bar{\nu}^{\alpha} & \\
& =e^{-3 \sigma}\left[\left(d_{(1)} k / d s\right)_{(2)} \nu^{\alpha}+{ }_{(1)} k_{(2)} k_{(3)} \nu^{\alpha}-\sigma_{B \gamma(1)} \nu^{\gamma} h^{\alpha \beta}\right]
\end{aligned}
$$

$\left.{ }^{70}\right)$ The Christoffel symbols $\left\{\begin{array}{l}1 \\ 1_{1}\end{array}\right\}, \Gamma^{1}{ }_{11}$ which occur in this section are formed with respect to $g_{i j}, G_{i j}$ respectively. 
where, according to (4.10) and (4.12),

$$
h^{\alpha \beta}=a^{\alpha \beta}-{ }_{(1)} \nu^{\alpha}{ }_{(1)} \nu^{\beta} .
$$

Now, if $m>2$, equations (11.3) may be solved for $\sigma_{\beta \gamma}$. If this result is substituted in (34.7) and use is made of (2.6) and (34.6), we obtain

$$
\bar{\Omega}^{2} \bar{\zeta}^{\alpha}=e^{-3 \sigma} \Omega^{2} \zeta^{\alpha}
$$

where

$$
\Omega^{2} \zeta^{\alpha}=\frac{d k_{1}}{d s}{ }_{(2)} \nu^{\alpha}+k_{1} k_{2(3)} \nu^{\alpha}+\frac{1}{(m-2)} \rho_{\beta \gamma(1)} \nu^{\gamma} h^{\alpha \beta}
$$

and $\bar{\Omega}^{2} \xi^{\alpha}$ is defined by analogous equations and $a_{\alpha \beta} \zeta^{\alpha} \zeta^{\beta}=1, \bar{a}_{\alpha \beta} \xi^{\alpha} \xi^{\beta}=1$. It is clear that $\zeta^{\alpha}$ is orthogonal to (1) $\nu^{\alpha}$. We assume that the members of (34.8) are not zero. Then the quantity $\Omega$ which is the unique positive root of $\Omega^{2}$ is a relative conformal scalar having the transformation law

$$
\bar{\Omega}=e^{-\sigma} \Omega \text {. }
$$

In the conformal theory of a curve, the quantity $\Omega$ occupies the role that is played by $\Lambda$ in the conformal theory of subspaces whose dimensionality exceeds 1 . We therefore call $\Omega$ the relative conformal curvature of $V_{1}$. The scalar $\Omega$ may be used to develop the curve theory in a manner analogous to the corresponding use of $\Lambda$ in the theory of $V_{n}$ with $n>1$. The analogy applies to all methods and results which only depend upon the transformation law (6.13) of $\Lambda$ and make no use of the actual definition of $\Lambda$. Because of the presence of the term $\rho_{\beta \gamma}{ }_{(1)} \nu^{\gamma} h^{\alpha \beta} /(m-2)$ in the definition of $\Omega$ the conformal geometry of a curve is simplified considerably if the enveloping $V_{m}$ is an Einstein space. On the other hand, Einstein spaces do not occupy any special or preferred position in the conformal theory of a subspace $V_{n}$ whose dimensionality $n$ exceeds 1 .

It follows from (2.6), (6.2) and (34.10) that the tensors

$$
G_{11}=\Omega^{2} g_{11}, \quad A_{\alpha \beta}=\Omega^{2} a_{\alpha \beta}
$$

are positive definite tensors which remain unchanged by conformal transformations of $V_{m}$. We call them the conformal measure tensors of $V_{1}$ and $V_{m}$ respectively and measure conformal length by means of these tensors. The conformal coefficients of connection analogous to (8.5) and (8.11) are

$$
\begin{aligned}
\Gamma^{1_{11}}= & \left\{\begin{array}{c}
1 \\
11
\end{array}\right\}+[\log \Omega]_{, 1}=\left[\log \left(g_{11}\right)^{1 / 2} \cdot \Omega\right]_{, 1}, \\
\Gamma_{\beta \gamma}^{\alpha_{\beta \gamma}=} & \left\{\begin{array}{c}
\alpha \\
\beta \gamma
\end{array}\right\}+a_{\beta}^{\alpha}\left[\mu_{\gamma}+{ }_{(1)} \nu_{\gamma} \frac{d \log \Omega}{d s}\right] \\
& +a_{\gamma}^{\alpha}\left[\mu_{\beta}+{ }_{(1)} \nu_{\beta} \frac{d \log \Omega}{d s}\right]-a_{\beta \gamma}\left[\mu^{\alpha}+{ }_{(1)} \nu^{\alpha} \frac{d \log \Omega}{d s}\right] .
\end{aligned}
$$


Conformal differentiation with respect to $x^{1}$ is defined by means of these coefficients as in (8.1). The various italicized statements of $\S 8$ are also true in the curve theory.

We define $S$ (up to an additive constant and choice of sign) by the equation

$$
S=\int\left(G_{11}\right)^{1 / 2} d x^{1}=\int \Omega d s .
$$

It is clear that $S$ remains invariant under conformal transformations and is a conformal scalar. We call $S$ a conformal arc length parameter of $V_{1}$. If the values of the same arc length parameter are $S_{1}$ and $S_{2}$ at two points $P_{1}$ and $\Phi_{2}$ of $V_{1}$, we call $\left|S_{1}-S_{2}\right|$ the conformal arc length of the $\operatorname{arc} \Phi_{1} \mathscr{P}_{2}\left(\right.$ or $\mathcal{P}_{2} \mathscr{P}_{1}$ ) of $V_{1}$. It is clear that the conformal arc length is independent of the choice of conformal arc length parameter and remains unchanged by conformal transformations of $V_{m}$. It follows from (34.11) and (34.12) that if $x^{1}=S$,

$$
G_{11}=1, \quad \Gamma^{1}{ }_{11}=0,
$$

so that conformal differentiation with respect to $S$ is defined by means of $\Gamma_{\beta \gamma}^{\alpha}$ alone. If we use the symbol $\vartheta / \vartheta S$ to denote conformal differentiation with respect to $S$,

$$
\begin{aligned}
\frac{\vartheta}{\vartheta S} T^{\alpha_{1} \cdots \alpha_{w_{\beta_{1}}} \ldots \beta_{v}}= & \frac{d}{d S} T^{\alpha_{1} \cdots \alpha_{w_{\beta_{1}}} \ldots \beta_{v}}+\sum_{a=1}^{w} T^{\alpha_{1} \cdots \alpha_{a-1} \gamma \alpha_{a+1} \cdots \alpha_{w_{1}} \cdots \beta_{v}} \Gamma^{\alpha_{a}}{ }_{\gamma \delta} \frac{d y^{\delta}}{d S} \\
& -\sum_{b=1}^{1} T^{\alpha_{1} \cdots \alpha_{w_{\beta_{1}}} \cdots \beta_{b-1} \gamma \beta_{b+1} \cdots \beta_{v}} \Gamma^{\gamma_{\beta_{v} \delta}} \frac{d y^{\delta}}{d S} .
\end{aligned}
$$

The vector (1) $Z^{\alpha}$ defined by

$$
{ }_{(1)} Z^{\alpha}=\Omega^{-1}(1) \zeta^{\alpha}
$$

is a conformal vector of unit conformal length. We call ${ }_{(1)} Z^{\alpha}$ the first conformal normal of $V_{1}$. Suppose that the class of ${ }_{(1)} Z^{\alpha}$ is $C^{1}$ and that this is also true of each successive unit conformal vector ${ }_{(2)} Z^{\alpha},{ }_{(3)} Z^{\alpha}, \cdots,{ }_{(u)} Z^{\alpha}$ obtained from ${ }_{(1)} Z^{\alpha}$ by the Frenet process $\left({ }^{71}\right)$ coupled with conformal differentiation. Then one readily proves, using the usual argument by mathematical induction, that there exists a set of scalars ${ }_{(1)} J,_{(2)} J, \cdots,{ }_{(u-1)} J(u \leqq n-1)$ such that

$$
\begin{gathered}
\frac{\vartheta_{(v)} Z^{\alpha}}{\vartheta S}=-{ }_{(v-1)} J_{(v-1)} Z^{\alpha}+{ }_{(v)} J_{(v+1)} Z^{\alpha}, \quad{ }_{(0)} J={ }_{(u)} J=0, \quad v=1,2, \cdots, u, \\
A_{\alpha \beta(v)} Z^{\alpha}{ }_{(w)} Z^{\beta}=\delta^{v}{ }_{w}, \quad v, w=1,2, \cdots, u, \\
A_{\alpha \beta(v)} Z^{\alpha}{ }_{(1)} \nu^{\beta}=0 .
\end{gathered}
$$

(71) The application of the Frenet process to the unit conformal tangent vector (in a manner analogous to that used in Riemannian geometry as well as in the conformal theory of a subspace $V_{n}$ with $\left.n>2\right)$ is unfruitful. For it is easy to verify that $\vartheta\left(\Omega^{-1}(i) \nu^{\alpha}\right) / \vartheta S=0$. 
The conformal unit vector ${ }_{(v)} Z^{\alpha}$ is called the vth conformal normal of $V_{1}$ and the conformal scalar ${ }_{(v)} J$ is called the vth conformal curvature of $V_{1}$. Equations (34.15) are called the conformal Frenet equations of $V_{1}$. If $V_{m} \rightleftarrows \bar{V}_{m}, V_{1} \rightleftarrows \bar{V}_{1}$ by a conformal transformation, all the quantities in (34.15) remain unchanged and (34.15) are also valid for $\bar{V}_{1}$ in $\bar{V}_{m}$.

The conformal unit vectors

$$
\Omega^{-1}{ }_{(1)} \nu^{\alpha},{ }_{(1)} Z^{\alpha},{ }_{(2)} Z^{\alpha}, \cdots,{ }_{(u)} Z^{\alpha}
$$

have roles analogous to the basis beins $G^{\alpha}{ }_{\left({ }_{i w}\right)}$ of the general subspace theory. The conformal vector $\Omega^{-1}$ (1) $\nu^{\alpha}$ spans the tangent vector space of $V_{1}$ and each normal vector ${ }_{(v)} Z^{\alpha}$ in (34.16) spans a one-dimensional vector space called the vth conformal normal vector space of $V_{1}$. The successive conformal curvatures of $V_{1}$ take the place of the various conformal normal measure tensors $B_{\left(i_{2}\right) \mid\left(h_{2}\right)}$, $B_{\left(i_{3}\right) \mid\left(h_{3}\right)}, \cdots, B_{\left(i_{M}\right) \mid\left(h_{M}\right)}$ of chapter IV.

Corresponding to the deviation tensor $E_{i j}$, we may construct the conformal tensor $J_{i j}$, defined analogously to (13.14), by the equation

$$
\begin{aligned}
J_{i j}= & (-\log \Omega)_{: i j}-(-\log \Omega)_{: i}(-\log \Omega)_{: j}+\mu_{\alpha} G^{\alpha}{ }_{i j} \\
& -\frac{1}{(m-2)} \rho_{\alpha \beta} G^{\alpha} G_{i}{ }_{j}+\frac{1}{2}\left[\Omega^{-2} \mu^{2}+G^{h k}(-\log \Omega)_{: h}(-\log \Omega)_{: k}\right. \\
& \left.+\frac{\Omega^{-2} \rho}{(m-1)(m-2)}\right] G_{i j} .
\end{aligned}
$$

To obtain this equation, we simply replace $\Lambda$ by $\Omega$ and $E_{i j}$ by $J_{i j}$ in (13.14). Since $i=j=1$, the tensor $J_{i j}$ has only one component so that we may consider the equivalent conformal invariant $J_{i j} G^{i j}$. Since $G^{\alpha}{ }_{i j} \equiv 0$ and $G_{i j} G^{i j}=1$ for $V_{1}$, we obtain from (34.17),

$$
J_{i j} G^{i j}=\Omega^{-2}\left[-\Omega \Delta_{2} \Omega+\Delta_{1} \Omega / 2+\mu^{2} / 2\right.
$$

$$
\left.+\frac{\rho}{2(m-1)(m-2)}-\frac{1}{(m-2)} \rho_{\alpha \beta} g^{\alpha \beta}\right]
$$

where $\Delta_{2} \Omega=G^{i j} \Omega_{: i j}, \Delta_{1} \Omega=G^{i j} \Omega_{: i} \Omega_{: j}$. If we choose $x^{1}=S$ it is clear that $\Delta_{2} \Omega=d^{2} \Omega / d S^{2}, \Delta_{1} \Omega=(d \Omega / d S)^{2}$. Also $g^{\alpha \beta}={ }_{(1)} \nu^{\alpha}{ }_{(1)} \nu^{\beta}$. If these substitutions are made in (34.18), we find that

$$
-2 J_{i j} G^{i j}={ }_{(m-1)} J
$$

where $_{(m-1)} J$ is the $(m-1)$ th conformal curvature of $V_{1}$ defined in our previous paper on curve theory as $\left({ }^{72}\right)$

$$
{ }_{(m-1)} J=\left[2 \Omega \frac{d^{2} \Omega}{d S^{2}}-\left(\frac{d \Omega}{d S}\right)^{2}-\left(k^{2}{ }_{1}+v\right)\right] / \Omega^{2}
$$

(72) Equation (34.20) corrects a misprint in our paper on curve theory [8, equation (5.10)]. 
where $v$ is defined by the equation

$$
v=(1 /(m-1)(m-2))\left[\rho-2(m-1) \rho_{\alpha \beta(1)} \nu^{\alpha}{ }_{(1)} \nu^{\beta}\right] .
$$

It follows from (34.19) and the invariance of $J_{i j} G^{i j}$ that ${ }_{(m-1)} J$ is a conformal invariant of $V_{1} . J_{i j} G^{i j}$ plays a role in the conformal theory of a curve exactly analogous to that of $E_{i j}$ in the conformal geometry of $V_{n}$ with $n>1$.

The conformal invariants $S,_{(1)} J,_{(2)} J, \cdots,_{(u-1)} J,_{(m-1)} J$ and the process of conformal differentiation constitute the foundation upon which the conformal geometry of a curve rests. While many of the results of $\$ \$ 10-17$ and of chapter $\mathrm{V}$ of this paper are vacuous or trivial in the curve theory, the remaining portions of the paper are similar to the corresponding results obtained by similar proofs in the conformal geometry of a curve. For example, the analogue of Theorem 19.1 is: If the conformal curvatures ${ }_{(1)} J_{\text {(2) }} J, \cdots,_{(u-1)} J_{(m-1)} J$ exist for a curve $V_{1}$ of class $C^{3}$ in a $V_{m}(m>2)$, then the conformal curvatures also exist for the conformal image curve $\bar{V}_{1}$ in $\bar{V}_{m}$. It is possible to choose a conformal arc length parameter $S$ so that corresponding points of $V_{1}$ and $\vec{V}_{1}$ have the same value of $S$ and the corresponding conformal curvatures are the same funciions of $S$ for $V_{1}$ and $\bar{V}_{1}$.

The existence theorem is true in a stronger form than obtains in the general subspace theory. For, if $n=1$, the system of partial differential equations (29.4) is replaced by a system of ordinary differential equations. The continuity of the functions ${ }_{(1)} J(S){ }_{{ }_{(2)}} J(S), \cdots,{ }_{(u-1)} J(S),{ }_{(m-1)} J(S)$ is then sufficient to insure the existence of a curve $V_{1}$ in any $V_{m}$ which has these functions as its conformal curvatures and one of whose conformal arc length parameters is $S$. This curve is uniquely determined by a set of initial conditions which may be given explicitly. If $V_{m}$ is an $\bar{R}_{m}$, analogues of the fundamental conformal equivalence theorem and the theorem on conformal differential invariants (Theorem 32.1) exist in the curve theory. Finally, we note that the assumption $m>2$ may be waived for the curve theory if we restrict ourselves to Riemann spaces $V_{m}$ and to special conformal transformations of $V_{m}$ which have the property that any geodesic circle of $V_{m}$ maps upon a geodesic circle $\left({ }^{73}\right)$ of $\bar{V}_{m}$. These transformations are generalizations of the inversive transformations of the plane $R_{\mathbf{2}}$. The reader is referred to our paper on the conformal theory of curves [8] for the detailed proofs of the various matters considered in this section as well as other subjects in the conformal geometry of a curve.

\section{BibliograPHY}

1. L. Bianchi, Lezioni di Geometrie Differenziale, 2nd ed., vol. 1, 1902.

2. W. Blaschke and G. Thomsen, Vorlesungen über Differentialgeometrie, vol. 3, 1929.

3. A. Duschek and W. Mayer, Lehrbuch der Differentialgeometrie, vol. 2, 1930.

${ }^{(73)}$ The conformal mapping function $\sigma$ for a transformation of this kind is a solution of the partial differential equations $\sigma_{\alpha \beta}=f \cdot a_{\alpha \beta}$ where $f$ is a point function. Some geometric properties of these transformations have been studied in $[8, \S \$ 13-14]$ and [6]. 
4. L. P. Eisenhart, Riemannian geometry, 1926.

5. ——, Non-Riemannian geometry, Amer. Math. Soc. Colloquium Publications, vol. 8, 1927.

6. A. Fialkow, Conformal geodesics, Trans. Amer. Math. Soc. vol. 45 (1939) pp. 443-473.

7. - The conformal theory of curves, Proc. Nat. Acad. Sci. U.S.A. vol. 26 (1940) pp. $437-439$. 501.

8. - The conformal theory of curves, Trans. Amer. Math. Soc. vol. 51 (1942) pp. 435-

9. S. Lie, Über Complexe, insbesondere Linien- und Kugelcomplexe, mit Anwendung auf die Theorie partieller Differentialgleichungen, Math. Ann. vol. 5 (1872) pp. 145-246.

10. W. Mayer, Die Differentialgeometrie der Untermannigfaltigkeiten des $R_{n}$ konstanter Krümmung, Trans. Amer. Math. Soc. vol. 38 (1935) pp. 267-309.

11. W. Mayer and T. Y. Thomas, Vollständig integrable Systeme totaler Differentialgleichungen, Math. Zeit. vol. 40 (1936) pp. 658-661.

12. J. A. Schouten, Über die konforme Abbildung n-dimensionaler Mannigfaltigkeiten mit quadratischer Massbestimmung auf eine Mannigfaltigkeit mit euclidischer Massbestimmung, Math. Zeit. vol. 11 (1921) pp. 58-88.

13. J. A. Schouten and D. J.-Struik, Einführung in die neueren Methoden der Differentialgeometrie, vol. 2, 1938.

14. T. Takasu, Differentialgeometrien in den Kugelräumen, vol. 1, 1938.

15. T. Y. Thomas, The differential invarianis of generalized spaces, 1934.

16. - Recent trends in geometry, Amer. Math. Soc. Semicentennial Publications, vol. 2 (1938) pp. 98-135.

17. H. Weyl, Reine Infinitesimalgeometrie, Math. Zeit. vol. 2 (1918) pp. 384-411.

18. H. Whitney, Analytic extensions of differentiable functions defined in closed sets, Trans. Amer. Math. Soc. vol. 36 (1934) pp. 63-89.

Columbia University, NEw YORK, N. Y. 